\title{
Die Methode von Smolyak bei der multivariaten Interpolation
}

\author{
Dissertation \\ zur Erlangung des Doktorgrades \\ der Mathematisch-Naturwissenschaftlichen Fakultäten \\ der Georg-August-Universität zu Göttingen
}

\author{
vorgelegt von \\ Anja Schreiber \\ aus Uslar
}

Göttingen 2000 


\section{7}

Referent: Prof. Dr. R. Schaback

Korreferent: Prof. Dr. J. Werner

Tag der mündlichen Prüfung: 22. Juni 2000 


\section{Inhaltsverzeichnis}

1 Einleitung 4

2 Interpolation mit Basisfunktionen $\quad 10$

2.1 Fehlerabschätzungen ..................... 12

2.2 Fehlerabschätzungen bei radialen Basisfunktionen . . . . . . . . 14

2.3 Native Spaces . . . . . . . . . . . . . . . 15

3 Der Smolyak-Algorithmus $\quad 18$

3.1 Tensoren und Tensorprodukte . . . . . . . . . . . . . 19

3.2 Bildung und Eigenschaften des Smolyak-Tensorprodukt-Algorithmus . 27

3.3 Das dünne Gitter und die Anzahl seiner Punkte . . . . . . . . . . . 30

3.4 Smolyakfunktionen ..................... 41

4 Interpolation auf dem dünnen Gitter $\quad 45$

4.1 Untersuchung geeigneter Funktionenräume und ihrer Normen . . . . . 49

4.2 Untersuchung geeigneter Native Spaces . . . . . . . . . . 64

4.3 Polynom-Interpolation . . . . . . . . . . . . . . 68

4.4 Interpolation mit Splines . . . . . . . . . . . . . 70

4.5 Interpolation mit Basisfunktionen . . . . . . . . . . 75

$5 \quad$ Interpolation auf dem gestörten dünnen Gitter 83

5.1 Bedingte positive Definitheit im Smolyak-Polynomraum . . . . . . . . 84

5.2 Das gestörte dünne Gitter . . . . . . . . . . . . . . 88

5.3 Fehlerabschätzungen . . . . . . . . . . . . . . 92

5.3.1 Allgemeine Basisfunktionen ............. 95

5.3.2 Tensorprodukt-Basisfunktionen ............. 98

5.3.3 Tensorprodukt-Basisfunktionen auf dem dünnen Gitter . . . . 101 
5.3.4 Smolyak-Basisfunktionen . . . . . . . . . . . . . . . . . . 104

5.3.5 Praktische Fehlerschranken . . . . . . . . . . . . . . . 107

5.4 Auswertung . . . . . . . . . . . . . . . . . . . . . . . . . . . 119

5.4 .1 Wendlandfunktionen . . . . . . . . . . . . . . . . 119

5.4 .2 Gaußglocken . . . . . . . . . . . . . . . . 120

5.4 .3 Inverse Multiquadrics . . . . . . . . . . . . . . 123

5.4 .4 Multiquadrics . . . . . . . . . . . . . . . 125

5.4 .5 Thin Plate Splines . . . . . . . . . . . . . . 127

5.4 .6 Polynomials . . . . . . . . . . . . . . . . . . . . . 129

$\begin{array}{ll}\text { Symbolverzeichnis } & 130\end{array}$

$\begin{array}{ll}\text { Literatur } & 133\end{array}$

$\begin{array}{ll}\text { A Anhang } & 137\end{array}$ 


\section{Einleitung}

In einer Vielzahl von Anwendungen aus Wissenschaft, Wirtschaft und Industrie besteht die Notwendigkeit, Funktionen aus vorgegebenen Daten zu rekonstruieren. Dabei treten nicht selten auch Daten von Funktionen mit sehr vielen Variablen auf, die mit entsprechenden Interpolationsmethoden behandelt werden müssen.

Im Rahmen der multivariaten Approximationstheorie werden solche Problemstellungen wirkungsvoll durch die Interpolation mit Basisfunktionen gelöst. Im klassischen Fall verwendet man Basisfunktionen, die radial sind; eine derartige Vorgehensweise ist jedoch nicht zwingend erforderlich und auch nicht in jedem Fall zweckmäßig. Wir formulieren eine solche eindeutig bestimmte Interpolante $s_{f, \Phi, X}$ an eine Funktion $f$ auf dem Datensatz $X=\left\{x_{1}, \ldots, x_{N}\right\}$ mit zugrundegelegter Basisfunktion $\Phi$ mittels

$$
s_{f, \Phi, X}=\sum_{j=1}^{N} \alpha_{j} \Phi\left(\cdot, x_{j}\right)+p
$$

mit $p \in \mathcal{P}$ und

$$
\sum_{j=1}^{N} \alpha_{j} \tilde{p}\left(x_{j}\right)=0 \quad \text { für alle } \tilde{p} \in \mathcal{P}
$$

dabei muß die Basisfunktion $\Phi$ bedingt positiv definit bezüglich des Raumes $\mathcal{P}$ sein. Benutzt wird die Interpolation mit (radialen) Basisfunktionen beispielsweise zum Entwurf neuronaler Netze, zur Lösung von partiellen Differentialgleichungen [11] oder im Bereich des Computer Aided Design [32].

Bei der Interpolation in hohen Raumdimensionen führt jedoch ein auch aus vielen anderen Teilbereichen der numerischen Mathematik bekanntes Phänomen zu einem schwerwiegenden komplexitätstheoretischen Problem. Um in jeder Raumdimension gleichbleibend gute Resultate zu erzielen, muß der Daten- und damit auch der Rechenaufwand mit Erhöhung der Raumdimension in exponentieller Weise anwachsen. Dieser Sachverhalt wird deshalb auch als Fluch der Dimension bezeichnet. Im Rahmen der Interpolationstheorie nimmt dieser Fluch folgende Gestalt an: Bei der Reproduktion einer Funktion $f$ durch eine Interpolante $s_{f, X}$ auf dem Datensatz $X=\left\{x_{1}, \ldots, x_{N}\right\}$ messen wir die Qualität oder Approximationsgüte einer solchen numerischen Operation in vielen Fällen durch Beschränkung der Norm des Fehlerfunktionals

$$
\left\|f-s_{f, X}\right\| \leq C h_{X, \Omega}^{\beta}
$$

in Abhängigkeit von der Fülldistanz

$$
h_{X, \Omega}=\sup _{y \in \Omega} \min _{x \in X}\|x-y\|,
$$


einer Maßgröße für die Datenverteilungsdichte auf dem Gebiet $\Omega$. Man bezeichnte dann $\beta$ als Approximationsordnung. Ist der Datensatz $X$ quasi-regulär, d.h. in gleichmäßiger Art und Weise verteilt, so kann auf einem beschränkten Gebiet die Fülldistanz $h_{X, \Omega}$ in Beziehung zur Anzahl $N$ der Daten und der Raumdimension $d$ gesetzt werden. Die Norm des Fehlerfunktionals erhält dann eine obere Schranke der Form

$$
\left\|f-s_{f, X}\right\| \leq \tilde{C} N^{-\frac{\beta}{d}}
$$

und die Approximationsgüte verschlechtert sich deutlich mit anwachsender Raumdimension, wenn nicht die Anzahl der Daten entsprechend erhöht wird.

Eine Lösungsmöglichkeit für dieses Problem besteht in der Anwendung des Algorithmus von Smolyak [40], der 1960 für das Gebiet der numerischen Integration eine Methode entwickelt hat, welche dem Fluch der Dimension erheblich entgegenwirkt. Grundlage dieser Methode ist es, die Komplexität voller $d$-dimensionaler Tensorprodukte bzw. Tensorprodukt-Operatoren durch geschickt gewählte Kombination von Differenzen zu reduzieren. Voraussetzung für eine zielgerichtete Nutzung des Verfahrens ist eine hinreichend hohe Glätte der zugrundegelegten Funktionen.

Seit dieser Zeit wurde der Smolyak-Algorithmus auf eine Vielzahl von hochdimensionalen Problemstellungen angewandt. Dazu zählen etwa die Lösung von partiellen Differentialgleichungen [38], [16], die trigonometrische Interpolation und Interpolation mit Wavelets [41], die Polynominterpolation [5], naturgemäß die numerische Integration [40], [25], dabei insbesondere die Approximation von stochastischen Integralen [30], [23] und als Anwendung derselben die numerische Berechnung hochdimensionaler Integrale aus dem Finanzwesen zur Bewertung von Finanzderivaten. Alle diese Arbeiten benutzen einen speziellen Datensatz, der gitterartig strukturiert ist, aber gegenüber einem vollbesetzten Gitter eine deutlich geringere Punktanzahl aufweist und deshalb auch dünnes Gitter genannt wird.

In dieser Arbeit wird der Smolyak-Algorithmus zum ersten Mal bei der Interpolation mit Basisfunktionen angewandt. Es können dabei die verschiedensten Arten von Basisfunktionen, einschließlich solcher in radialer Form, verwandt werden. Als besonders geeignet erweist es sich allerdings, auf Basis von Tensorproduktfunktionen zu interpolieren. Das läßt sich unter anderem dadurch begründen, daß damit die Glätte der zugrundegelegten Basisfunktion im Gegensatz zur radialen Funktion gleichen Typs deutlich erhöht wird. Als Resultat erhalten wir verschiedene multivariate Interpolanten $s_{f, \Phi, X}$ an eine Funktion $f$ mit der Basisfunktion $\Phi$ auf dem Datensatz $X$, welche durch Verwendung des Smolyak-Algorithmus Fehlerabschätzungen der Form

$$
\left\|f-s_{f, \Phi, X}\right\|_{\infty} \leq \tilde{C} N^{-\beta}(2 d+\log (N))^{(d-1)(\beta+1)+c}
$$


folgern lassen; dabei bezeichnet $N$ die Anzahl der Punkte des Datensatzes. Bei gleichbleibener Datengröße ergeben sich damit Fehlerschranken, die im Vergleich mit eindimensionalen Ergebnissen fast optimal sind und sich mit Anwachsen der Raumdimension nicht wesentlich verschlechtern. Teilweise erreichen wir dabei sogar exponentielle Approximationsgüten; in diesen Fällen gilt

$$
\left\|f-s_{f, \Phi, X}\right\|_{\infty} \leq \tilde{C} e^{-\tilde{c}_{d} N(2 d+\log (N))^{d-1}}
$$

gegenüber klassischen Fehlerabschätzungen der Gestalt

$$
\left\|f-s_{f, \Phi, X}\right\|_{\infty} \leq C e^{-c N^{\frac{1}{d}}}
$$

in Abhängigkeit von der Größe $N$ eines regulären Datensatzes auf dem beschränkten Gebiet $\Omega$; durch Abschätzungen der obigen Form ist der Fluch der Dimension dort erheblich entschärft worden. Damit ist für das Komplexitätsproblem bei der Interpolation mit (radialen) Basisfunktionen eine brauchbare Lösung gefunden. Neuartig ist der mir bekannten Literatur zufolge auch die Nutzung der Methode auf einem allgemeineren, chaotischen Datensatz, denn der Smolyak-Algorithmus wird nicht mehr ausschließlich auf dem dünnen Gitter angewandt; daraus folgt eine deutlich erhöhte Anwendungsbreite des Verfahrens. Als Beispiel für eine Nutzung einer solchen Smolyak-Interpolation mit Basisfunktionen könnte unter anderem möglicherweise die Approximation hochdimensionaler Integrale aus dem Finanzwesen in Frage kommen; dort werden bisher Smolyak-Polynominterpolanten [27] auf dünnen Gittern als Hilfsmittel genutzt.
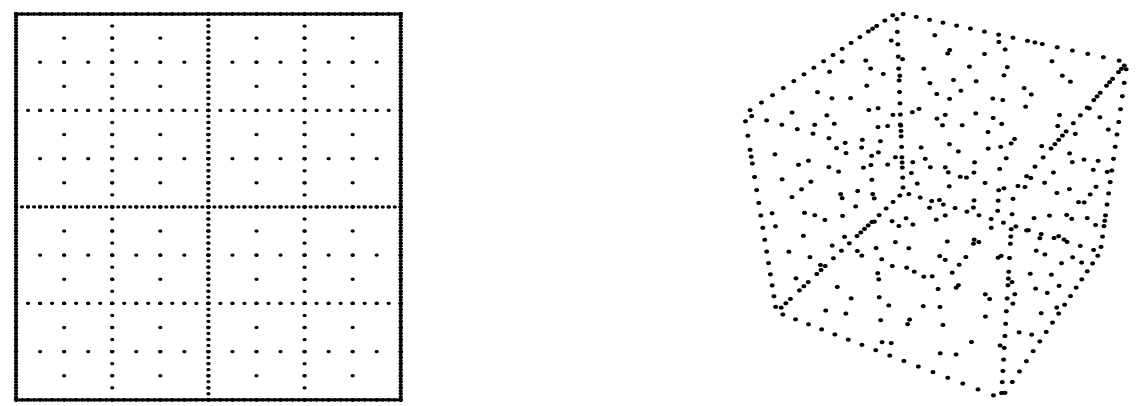

Abb.: Zweidimensionales und dreidimensionales dünnes Gitter 
Der Aufbau der Arbeit gliedert sich folgendermaßen:

Abschnitt 2 enthält die Grundlagen der Interpolation mit (radialen) Basisfunktionen. Dabei wird insbesondere auf die später besonders relevanten Themen wie die Methoden der Fehlerabschätzung und die Theorie der Räume der zu interpolierenden Funktionen, den sogenannten Native Spaces, eingegangen.

Abschnitt 3 ist der Methode von Smolyak gewidmet. Dort wird der Smolyak-Algorithmus, der sowohl bei Datenmengen, Funktionen, Funktionenräumen und lineare Operatoren eingesetzt werden kann, zunächst in einer sehr allgemeinen Gestalt und schließlich in den verschiedenen Anwendungen vorgestellt. Da für die Nutzung des Smolyak-Algorithmus die Theorie der Tensorprodukte und Tensorräume von entscheidender Bedeutung ist, wird sie hier ausführlich behandelt. Es folgt die Beschreibung des Smolyak-Tensorproduktoperators in seinen verschiedenen Darstellungsformen; er wird später für die Interpolation genutzt. Besondere Beachtung findet auch der zugrundegelegte Datensatz, das dünne Gitter. Da die zu erwartenden Fehlerabschätzungen in Abhängigkeit von seiner Punktanzahl dargestellt werden sollen, kommt ihrer Bestimmung erhöhte Bedeutung zu. Aus diesem Grund enthält der Abschnitt auch eigene Ergebnisse zu dieser Fragestellung, und insgesamt wurde auf die detallierte Darstellung der Konstanten in Abhängigkeit von der Raumdimension viel Wert gelegt, um diese Information an die späteren Fehlerabschätzungen weitergeben zu können. Zudem betrachten wir einen neuen Typ von multivariaten Funktionen, den wir gewinnen, wenn wir den Smolyak-Algorithmus auf univariate Funktionen anwenden. Wichtige Beispiele für solche Funktionen sind multivariate Polynome, deren Monombasen ebenfalls einer Smolyak-Struktur folgen und die wir deshalb als Smolyak-Polynome bezeichnen wollen.

Auf Basis der im vorangegangenen Abschnitt bereitgestellten theoretischen Grundlagen wird in Abschnitt 4 in allgemeiner und ausführlicher Form festgestellt, unter welchen Umständen die Methode von Smolyak genutzt werden kann und unter welchen Bedingungen sie den Fluch der Dimension behebt. Dabei wird deutlich, daß die Nutzbarkeit des Algorithmus im wesentlichen auf die Struktur der zugrundegelegten Funktionenräume und ihrer mit einer besonderen Eigenschaft ausgestatteten Normen zurückzuführen ist. Dementsprechend enthält dieser Abschnitt eine Sammlung von Funktionenräumen, die für die Verwendung des Smolyak-Algorithmus geeignet sind. Darauf aufbauend ist die Anwendbarkeit der Smolyak-Methode auf spezielle Interpolanten wie etwa auf Polynome nach [5] oder auf eine Vielzahl von Splines sofort nachvollziehbar. Für letzteres Interpolationsproblem werden entsprechende Fehlerschranken bereitgestellt, die schließlich keinem Fluch der Dimension mehr unterliegen.

Besondere Aufmerksamkeit wird naturgemäß der Fragestellung gewidmet, inwiefern 
die der Interpolation mit Basisfunktionen zugrundegelegten Funktionenräume, die Native Spaces, ebenfalls den beschriebenen Bedingungen genügen, welche eine Nutzung der Smolyak-Methode zur Behebung des Fluches der Dimension ermöglichen. Eine Untersuchung der Strukturen der Native Spaces führt zu dem Ergebnis, daß wir dem Komplexitätsproblem entgegenwirken können, wenn wir mit Tensorprodukten einer univariaten, positiv definiten Basisfunktion interpolieren. Angewandt auf das Tensorprodukt von Wendlandfunktionen wird erwartungsgemäß eine in Abhängigkeit von der Anzahl der Datenpunkte des dünnen Gitters in jeder Raumdimension konstante polynomiale Approximationsgüte erreicht, unwesentlich abgeschwächt durch einen logarithmischen Faktor. Im Fall der Interpolation mit Gaußglocken oder tensorierten Inversen Multiquadrics gewinnen wir in Abhängigkeit von der Datenpunktanzahl sogar exponentielle Approximationsgüte, bei der der Fluch der Dimension erheblich abgemildert werden konnte.

Voraussetzung für alle diese Ergebnisse ist die Verwendung des dünnen Gitters als Datensatz. In Abschnitt 5 wird der Smolyak-Algorithmus in einer modifizierten, nicht der üblicherweise verwandten Methode folgenden Form benutzt. Dadurch wird erreicht, daß auf einem chaotischen verteilten Datensatz interpoliert werden kann. Dieser Datensatz verhält sich bezüglich der Verteilungsdichte seiner Punkte analog zum dünnen Gitter und wird deshalb gestörtes dünnes Gitter genannt. Eine weitere deutliche Verallgemeinerung gegenüber dem vorangegangenen Abschnitt besteht in der Möglichkeit, mit einer wesentlich größeren Menge von Basisfunktionen zu arbeiten. Der Grund für diese Verbesserungen liegt in der Wahl der Interpolante, die im Vergleich mit der herkömmlichen Interpolation mit (radialen) Basisfunktionen anstelle eines klassischen Polynomanteils ein Smolyak-Polynom enthält. Dadurch finden wir für allgemeine, bezüglich des Smolyak-Polynomraumes bedingt positiv definite Basisfunktionen Fehlerabschätzungen, die auf Approximationsfehler in diesem Polynomraum zurückzuführen sind und die mit Hilfe der Resultate aus Abschnitt 3 zur Beseitigung des Fluches der Dimension genutzt werden können. Bei Verwendung von Tensorprodukt-Basisfunktionen erhalten wir schließlich ohne Gebrauch der Ergebnisse des vorangegangenen Abschnittes sowohl direkt auf dem dünnen Gitter als auch auf dem gestörten dünnen Gitter weitere Fehlerschranken. Diese stellen verglichen mit dem ersten Resultat für allgemeine Basisfunktionen eine Verbesserung dar. Wie auch in Abschnitt 3 können wir damit dem Fluch der Dimension für die positiv definiten Tensor-Wendlandfunktionen, Gaußglocken und tensorierten Inversen Multiquadrics entgegenwirken, wobei allerdings auf dem chaotischen Datensatz noch keine exponentielle Konvergenz nachgewiesen werden kann. Nach gründlicher Untersuchung der bedingten positiven Definitheit im Smolyak-Polynomraum können schließlich auch Multiquadrics, Thin Plate Splines und Polynomials zur Interpolation herangezogen werden. Im Tensorproduktfall erreichen wir für alle diese Funktionen von der Anzahl der Daten abhängige Fehlerschranken, welche sich mit anwachsender Raumdimensi- 
on bei gleichbleibender Größe des Datensatzes nicht verschlechtern, sondern sogar in allen diesen Fällen zu einer gegenüber einer klassischen eindimensionalen Fehlerschranke verbesserten Approximationsgüte führen.

Die in dieser Arbeit verwendeten Symbole entsprechen den in der Mathematik allgemein üblichen Bezeichnungen. Zur Begriffsklärung finden sich weiterführende Notationen im Symbolverzeichnis.

Für die Betreuung dieser Dissertation möchte ich mich ganz herzlich bei meinem Doktorvater, Herrn Prof. Dr. Robert Schaback, bedanken. Weiterhin danke ich Herrn Prof. Dr. Jochen Werner für die Übernahme des Korreferates und der Graduiertenförderung des Landes Niedersachsen für die Finanzierung meiner Arbeit. Besonderer Dank gebührt meiner Familie für ihre Unterstützung während des gesamten Studiums und beim Entstehen dieser Dissertation.

Göttingen, im Mai 2000

Anja Schreiber 


\section{Interpolation mit Basisfunktionen}

Bei gegebenem beliebig chaotisch verteiltem Datensatz $X=\left\{x_{1}, \ldots, x_{N}\right\}$ mit $x_{j} \in \mathbb{R}^{d}$ und gegebener Funktion $\Phi: \mathbb{R}^{d} \rightarrow \mathbb{R}$ suchen wir zur Funktion $f: \mathbb{R}^{d} \rightarrow \mathbb{R}$ eine Interpolante der Gestalt

$$
s_{f, \Phi, X}(x)=\sum_{j=1}^{N} \alpha_{j} \Phi\left(x-x_{j}\right)+\sum_{k=1}^{Q} \beta_{k} p_{k}(x)
$$

mit $s_{f, \Phi, X}\left(x_{j}\right)=f\left(x_{j}\right)=: y_{j}$ für alle $j$. Es gelte dabei $\alpha:=\left\{\alpha_{1}, \ldots, \alpha_{N}\right\}$, $\beta:=\left\{\beta_{1}, \ldots, \beta_{Q}\right\} \in \mathbb{R}^{d}$, wobei die Nebenbedingung

$$
\sum_{j=1}^{N} \alpha_{j} p_{k}\left(x_{j}\right)=0
$$

erfüllt sei; weiterhin bezeichne $\left\{p_{1}, \ldots, p_{Q}\right\}$ mit $p_{k}: \mathbb{R}^{d} \rightarrow \mathbb{R}$ eine Basis des Polynomraums $\mathcal{P}$. Wir erhalten mit Hilfe der Matrizen $A_{\Phi, X}:=\left(\Phi\left(x_{j}-x_{l}\right)\right)_{1 \leq j, l \leq N}$ und $P_{X}=\left(p_{k}\left(x_{j}\right)\right)_{\substack{1 \leq j \leq N \\ 1 \leq k \leq Q}}$ das Gleichungssystem

$$
\left(\begin{array}{cc}
A_{\Phi, X} & P_{X} \\
P_{X}^{T} & 0
\end{array}\right)\left(\begin{array}{l}
\alpha \\
\beta
\end{array}\right)=\left(\begin{array}{l}
y \\
0
\end{array}\right),
$$

wobei $y=\left(y_{1}, \ldots, y_{N}\right)$ gelte.

Entscheidend für die Lösbarkeit dieses Gleichungssystems und damit für die Durchführbarkeit der Interpolation mit Basisfunktionen ist folgender Begriff.

Definition 2.1 Die Funktion $\Phi \in C\left(\mathbb{R}^{d}\right)$ heißt bedingt positiv definit bezüglich des Polynomraums $\mathcal{P}$ auf $\mathbb{R}^{d}$, wenn bei beliebiger Wahl von paarweise verschiedenen $x_{1}, \ldots, x_{N} \in \mathbb{R}^{d}$ und für alle $\alpha_{1}, \ldots, \alpha_{N} \in \mathbb{R}$ mit

$$
\sum_{j=1}^{N} \alpha_{j} p\left(x_{j}\right)=0
$$

für jedes $p \in \mathcal{P}$ die Bedingung

$$
\sum_{j=1}^{N} \sum_{k=1}^{N} \alpha_{j} \alpha_{k} \Phi\left(x_{j}-x_{k}\right) \geq 0
$$

mit Gleichheit nur im Fall $\alpha_{1}=\ldots=\alpha_{N}=0$ gilt. Die Funktion $\Phi$ heißt positiv definit, falls (2) für alle $\alpha_{1}, \ldots, \alpha_{N}$ gilt. 
Der Grund für die Wichtigkeit der bedingten positiven Definitheit ist der folgende

Satz 2.2 Ist die Basisfunktion $\Phi$ bedingt positiv definit bezüglich des Polynomraums $\mathcal{P}$ auf $\mathbb{R}^{d}$, so ist das lineare Gleichungssystem (1) bei injektiver Matrix $P_{X}$ eindeutig lösbar.

Der Beweis ist bei [22] zu finden.

Im klassischen, üblicherweise verwandten Fall benutzen wir den Polynomraum

$$
\mathbb{P}_{m}^{d}:=\operatorname{span}\left\{x_{1}^{j_{1}} \cdot \ldots \cdot x_{d}^{j_{d}} \quad: \quad j=\left(j_{1}, \ldots, j_{d}\right) \in \mathbb{N}_{0}^{d},|j| \leq m-1\right\}
$$

und formulieren:

Definition 2.3 Die Funktion $\Phi \in C\left(\mathbb{R}^{d}\right)$ heißt bedingt positiv definit der Ordnung $m$ auf $\mathbb{R}^{d}$, wenn sie bedingt positiv definit bezüglich des $\mathbb{P}_{m}^{d}$ auf $\mathbb{R}^{d}$ ist. Wir nennen sie bedingt positiv definit der Ordnung 0, falls sie positiv definit ist.

Um im klassischen Fall eine Funktion auf bedingte positive Definitheit zu überprüfen, bietet sich ein Ergebnis von Iske [15] an, welches eine Verallgemeinerung des Satzes von Bochner [6] darstellt:

Satz 2.4 Die stetige Funktion $\Phi: \mathbb{R}^{d} \rightarrow \mathbb{R}$ besitze eine verallgemeinerte Fouriertransformierte $\hat{\Phi}: \mathbb{R}^{d} \rightarrow \mathbb{R}$, für die eine reelle Zahl s existiert, so daß $\hat{\Phi}(\omega)\|\omega\|^{s}$ um Null integrierbar ist. Dann ist $\Phi$ genau dann bedingt positiv definit der Ordnung $m \geq \frac{s}{2}$ auf $\mathbb{R}^{d}$, wenn $\hat{\Phi}(\omega) \geq 0$ für alle $\omega \in \mathbb{R}^{d} \backslash\{0\}$ und $\hat{\Phi} \not \equiv 0$ erfüllt ist.

Aus diesen Sätzen erhalten wir für Funktionen mit gewöhnlicher Fouriertransformierter, vgl. [47]:

Satz 2.5 Eine stetige Funktion $\Phi \in L_{1}\left(\mathbb{R}^{d}\right)$ ist genau dann positiv definit, wenn für ihre Fouriertransformierte die Bedingungen $\hat{\Phi}(\omega) \geq 0$ für alle $\omega \in \mathbb{R}^{d} \backslash\{0\}$ und $\hat{\Phi} \not \equiv 0$ gelten.

Im Tensorproduktfall folgt daraus

Lemma 2.6 Sei $\Phi$ die Tensorproduktfunktion stetiger, auf $\mathbb{R}^{d_{j}}$ positiv definiter Funktionen $\phi_{j} \in L_{1}\left(\mathbb{R}^{r_{j}}\right), 1 \leq j \leq b$, mit $\sum_{j=1}^{b} r_{j}=d$. Dann ist $\Phi$ positiv definit auf $\mathbb{R}^{d}$. 
Beweis: Offenbar ist $\Phi$ stetig und entstammt dem Raum der Lebesgue-integrierbaren Funktionen $L_{1}\left(\mathbb{R}^{d}\right)$. Die (gewöhnlichen) Fouriertransformierten der jeweiligen Funktionen bezeichnen wir mit $\hat{\Phi}$ bzw. $\hat{\phi}_{j}$. Wenden wir nun Satz 2.5 auf die Funktionen $\phi_{j}$ und ihr Tensorprodukt $\Phi$ an, so folgt wegen $\hat{\Phi}=\hat{\phi}_{1} \otimes \ldots \otimes \hat{\phi}_{b}$ die Behauptung. $\square$

Dieses Ergebnis wird sich in einem späteren Abschnitt noch erweitern lassen.

\section{$2.1 \quad$ Fehlerabschätzungen}

Die Interpolante kann nach Basiswechsel auch in ihrer Lagrangedarstellung

$$
s_{f, \Phi, X}=\sum_{j=1}^{N} u_{j} f\left(x_{j}\right)
$$

geschrieben werden. Sind dabei beliebige Lagrangefunktionen $u_{j}$ zugelassen, für die folglich nicht unbedingt $s_{f, \Phi, X}\left(x_{j}\right)=f\left(x_{j}\right)$ gelten muß, so spricht man von QuasiInterpolation. Den Interpolationsfehler können wir nun mit Hilfe des Deltafunktionals $\delta_{y} f:=f(y)$ für alle $y \in \mathbb{R}^{d}$ darstellen:

$$
f(x)-s_{f, \Phi, X}(x)=\left(\delta_{x}-\sum_{j=1}^{N} u_{j}(x) \delta_{x_{j}}\right) f .
$$

Wir bezeichnen das Fehlerfunktional der Quasi-Interpolante im Punkt $x$ durch

$$
\lambda_{x, X, u}:=\delta_{x}-\sum_{j=1}^{N} u_{j}(x) \delta_{x_{j}}
$$

mit $u=\left\{u_{1}, \ldots, u_{N}\right\}$; es gilt also $f(x)-s_{f, \Phi, X}(x)=\lambda_{x, X, u} f$ und die für die Interpolation nötige Nebenbedingung läßt sich nun durch die Gleichung $\lambda_{x, X, u} p=0$ für alle $p \in \mathcal{P}$ beschreiben. Damit ist also $\lambda_{x, X, u}$ im $\mathbb{R}$-Vektorraum

$$
\begin{aligned}
L_{\mathcal{P}}(\Omega)= & \mathcal{P}^{\perp} \\
= & \left\{\lambda: \lambda \text { lineares Funktional der Form } \lambda f=\sum_{i=1}^{N^{\lambda}} \alpha_{i}^{\lambda} f\left(x_{i}^{\lambda}\right) \text { auf } C(\Omega)\right. \\
& \text { mit } \Omega \subseteq X^{\lambda}=\left\{x_{1}^{\lambda}, \ldots, x_{n}^{\lambda}\right\}, X^{\lambda} \text { paarweise verschieden und unisolvent, } \\
& \left.\sum_{i=1}^{N^{\lambda}} \alpha_{i}^{\lambda} p\left(x_{i}^{\lambda}\right)=0 \text { für alle } p \in \mathcal{P}, \lambda \equiv 0 \text { oder } \alpha_{i}^{\lambda} \neq 0,1 \leq i \leq N_{\lambda}\right\}
\end{aligned}
$$


enthalten. Ist nun die Basisfunktion $\Phi$ bedingt positiv definit bezüglich $\mathcal{P}$, so ist für alle $\lambda, \mu \in \mathcal{P}^{\perp}$ durch

$$
(\lambda, \mu)_{\Phi}:=\lambda^{u} \mu^{v} \Phi(u, v)
$$

ein Skalarprodukt auf $\mathcal{P}^{\perp}$ erklärt. Genauso wird auf dem Funktionenraum

$$
L\left(\mathcal{P}^{\perp}\right)=\left\{f_{\lambda}=\lambda^{u} \Phi(\cdot, u), \lambda \in \mathcal{P}^{\perp}\right\}
$$

bei bijektivem $L: \lambda \rightarrow f_{\lambda}$ mittels $\left(f_{\lambda}, f_{\mu}\right)_{\Phi}:=(\lambda, \mu)_{\Phi}$ ein Skalarprodukt definiert. Für die dadurch induzierte Norm gilt

$$
\left\|\lambda_{x, X, u}\right\|_{\Phi}=\sup _{\substack{f_{\lambda} \in L(\mathcal{P} \perp) \\\left\|f_{\lambda}\right\|_{\Phi} \neq 0}} \frac{\left|f_{\lambda}(x)-\sum_{j=1}^{N} u_{j}(x) f_{\lambda}\left(x_{j}\right)\right|}{\left\|f_{\lambda}\right\|_{\Phi}} .
$$

Die Norm des Fehlerfunktionals im Punkt $x$ auf diesem Raum $P_{x, X, u}:=\left\|\lambda_{x, X, u}\right\|_{\Phi}$ heißt Power- oder Gütefunktion der Quasi-Interpolation $f \rightarrow \sum_{j=1}^{N} u_{j}(x) f\left(x_{j}\right)$ auf $L\left(\mathcal{P}^{\perp}\right)$ in $x$. Wir erhalten damit eine Fehlerabschätzung

$$
\left|f_{\lambda}(x)-\sum_{j=1}^{N} u_{j}(x) f_{\lambda}\left(x_{j}\right)\right| \leq\left\|f_{\lambda}\right\|_{\Phi} P_{x, X, u}
$$

wobei die durch $\lambda_{x, X, u}$ beschriebene Quasi-Interpolante exakt auf $\mathcal{P}$ sei.

Weiterhin wird auf dem Vektorraum $L\left(\mathcal{P}^{\perp}\right) \oplus \mathcal{P}$ mit $\left(f_{\lambda}, p\right):=0$ für $p \in \mathcal{P}, \lambda \in \mathcal{P}^{\perp}$ durch $(\cdot, \cdot)_{\Phi}$ eine symmetrische Bilinearform auf $L\left(\mathcal{P}^{\perp}\right) \oplus \mathcal{P}$ mit Kern $\mathcal{P}$ definiert. Der erste Faktor $\left\|f_{\lambda}\right\|_{\Phi}$ in der Fehlerabschätzung (4) wird schließlich unter allen Elementen aus $L\left(\mathcal{P}^{\perp}\right) \oplus \mathcal{P}$ in der zugehörigen Seminorm $\|\cdot\|_{\Phi}$ minimal, wenn man die Basisfunktionen-Interpolante zu $\Phi$ einsetzt. Zum Beweis dieses Sachverhaltes vgl. [33].

Für die weitere Abschätzung der Powerfunktion $P_{x, X, u}$ kann nun genutzt werden, daß sie bei gegebenem Datensatz $X$ durch solche Lagrangebasen $u=\left\{u_{1}, \ldots, u_{N}\right\}$, die aus den Lösungen des Interpolationsproblems mit der bezüglich $\mathcal{P}$ bedingt positiv definiten Basisfunktion $\Phi$ resultieren, minimiert wird, vgl. wieder [33]. Es gilt also für alle Lagrangebasen $\tilde{u}=\left\{\tilde{u}_{1}, \ldots, \tilde{u}_{N}\right\}$ mit

$$
\sum_{j=1}^{N} \tilde{u}_{j}(x) p(x)=0 \text { für alle } p \in \mathcal{P}
$$

die Ungleichung

$$
P_{x, X, u} \leq P_{x, X, \tilde{u}} .
$$

Diese Vorgehensweise wird im folgenden Abschnitt im klassischen Fall der Interpolation mit radialen Basisfunktionen, welche bezüglich des Polynomraumes $\mathbb{P}_{m}^{d}$ bedingt positiv definit sind, erläutert. 


\subsection{Fehlerabschätzungen bei radialen Basisfunktionen}

In diesem Abschnitt benutzen wir wie angekündigt solche Basisfunktionen, welche sowohl radial als auch auf allgemein übliche Weise bedingt positiv definit im Sinn von Definition 2.3 sind. Im folgenden wird eine Lagrangebasis $\tilde{u}$ mit Eigenschaft (5) ausgewählt, welche eine weitere Abschätzung der Powerfunktion $P_{x, X, \tilde{u}}$ ermöglicht. Dazu werden zunächst Aussagen über lokale Interpolation im klassischen multivariaten Polynomraum $\mathbb{P}_{M}^{d}$ gemacht:

Lemma 2.7 (Lokale Polynominterpolation) Es gelten folgende Aussagen:

(1) Die Polynominterpolation im $\mathbb{P}_{M}^{d}=\operatorname{span}\left\{x^{\alpha}:|\alpha| \leq M-1\right\}$ ist in den Punkten $\alpha,|\alpha| \leq M-1$ stets eindeutig möglich.

(2) Es gilt $\operatorname{det}\left(\alpha^{\beta}\right)_{0 \leq|\alpha|,|\beta| \leq M-1} \neq 0$.

(3) Es existiert $\epsilon_{0}>0$, so daß für alle $x_{\alpha}$ mit $\left\|x_{\alpha}-\alpha\right\| \leq \epsilon_{0}$ die Polynominterpolation im $\mathbb{P}_{M}^{d}$ eindeutig lösbar ist.

Die Gitterdaten werden nun mit Hilfe des folgenden Lemmas gestört, wodurch zu chaotisch verteilten Daten übergegangen werden kann:

Lemma 2.8 (Störungslemma) $Z u$ jedem $k \in \mathbb{N}$ und für alle $\zeta>0$ existieren Konstanten $h_{0}>0$, und $c_{1}, c_{2}>0$, so daß es für alle $x \in \mathbb{R}^{d}$, für jeden Datensatz $X=\left\{x_{1}, \ldots, x_{N}\right\} \subset \mathbb{R}^{d}$ und

$$
h_{\zeta}(x):=\sup _{\|y-x\| \leq \zeta} \min _{1 \leq j \leq N}\left\|y-x_{j}\right\| \leq h_{0}
$$

lokale Lagrangefunktionen $\tilde{u}_{1}(x), \ldots, \tilde{u}_{N}(x) \in \mathbb{R}$ gibt mit

(1) $\sum_{j=1}^{N} \tilde{u}_{j}(x) p\left(x_{j}\right)=p(x)$ für alle $p \in \mathbb{P}_{k}^{d}$,

(2) $\tilde{u}_{j}(x)=0$ für alle $j$ mit $\left\|x_{j}-x\right\|>c_{1} h_{\zeta}(x)$ und

(3) $\sum_{j=1}^{N}\left|\tilde{u}_{j}(x)\right| \leq c_{2}$

Beide Beweise sind etwa in [33] zu finden. Nun kann die Gütefunktion $P_{X, x, u}$ abgeschätzt werden. Da die in diesem Abschnitt betrachteten Basisfunktionen radial 
sind, ist es möglich, die univariate Funktion $F$ mit $\Phi(x)=\phi(\|x\|)=: F\left(\|x\|^{2}\right)$ zu bestimmen. Es bezeichne nun

$$
E(k, \phi, \epsilon):=\inf _{p \in \mathbb{P}_{k}^{1}}\|F-p\|_{[0, \epsilon], \infty} .
$$

Die Lösung dieser Approximationsaufgabe stellt einen wesentlichen Bestandteil der oberen Schranke für die Powerfunktion dar:

Satz 2.9 Die d-variate radiale Basisfunktion $\Phi$ sei bedingt positiv definit der Ordnung $m$. Dann gilt für alle Datensätze $X=\left\{x_{1}, \ldots, x_{N}\right\}$ und für jeden $\mathbb{R}^{d}$-wertigen Punkt $x$ mit

$$
h_{\zeta}(x)=\sup _{\|y-x\| \leq \zeta} \min _{1 \leq j \leq N}\left\|x_{j}-y\right\| \leq h_{0}=\frac{\zeta \epsilon_{0}}{M-1},
$$

$\epsilon_{0}<\frac{1}{2}$ und $N \geq M \geq m$ die Abschätzung der Gütefunktion

$$
P_{X, \tilde{u}, x}^{2} \leq\left(1+c_{2}\right)^{2} E\left(k, \phi, \epsilon^{2}\right)
$$

mit $\epsilon \geq 2 c_{1} h_{\zeta}(x), M \geq 2 k-1, c_{1}=1+\frac{M-1}{\epsilon_{0}}$ und der von $\epsilon_{0}$ und $M$ abhängigen Lebesgueschen Konstante $c_{2}$.

Entscheidend für den Beweis ist Eigenschaft (2) des Störungslemmas 2.8. Zur weiteren Abschätzung von (6) werden üblicherweise Jackson-Sätze aus der Approximationstheorie benutzt.

\subsection{Native Spaces}

Für spätere Anwendungen ist es notwendig, die Eigenschaften der der Interpolation mit Basisfunktionen zugrundeliegenden Funktionenräume zusammenzustellen. Die Beweise der zitierten Ergebnisse und Aussagen können - falls keine anderen Informationen gegeben werden - in [33] oder [34] nachgelesen werden.

Als grundlegend erweist sich bei der Untersuchung der Funktionenräume, die bei der Interpolation mit Basisfunktionen benutzt werden, der folgende Begriff.

Definition und Satz 2.10 Die Funktion $K: X \times X \rightarrow \mathbb{R}$ heißt Kernfunktion oder reproduzierender Kern eines Hilbertraumes $H_{K}$ von Funktionen auf $X$, falls $K(\cdot, y) \in H_{K}$ für alle $y \in X$ erfüllt ist und die Reproduktionseigenschaft

$$
f(y)=(f(x), K(x, y))_{H_{K}}
$$

für alle $x, y \in X$ und für alle $f \in H_{K}$ gilt. Mit $(\cdot, \cdot)_{H_{K}}$ bezeichnen wir dabei das Skalarprodukt von $H_{K}$. Die Kernfunktion eines Hilbertraumes ist eindeutig. 
Die Beweise sind beispielsweise bei [3], [21] oder [31] zu finden. Damit definieren wir zunächst:

Definition 2.11 Sei $\Phi: \Omega \times \Omega \rightarrow \mathbb{R}$ eine symmetrische, positiv definite Funktion, welche der reproduzierende Kern eines reellen Hilbertraumens $\mathcal{H}$ von reellwertigen Funktionen auf $\Omega$ ist. Dann heißt $\mathcal{N}_{\Phi}(\Omega):=\mathcal{H}$ Native Space von $\Phi$.

Sind keine Mißverständnisse zu erwarten, so schreiben wir auch $\mathcal{N}_{\Phi}$ anstelle von $\mathcal{N}_{\Phi}(\Omega)$. Wegen $(\Phi(x, \cdot), \Phi(y, \cdot))=\Phi(x, y)$ besteht der Native Space einer positiv definiten Funktion aus dem Abschluß $\mathcal{F}_{\Phi}(\Omega)$ des Raumes

$$
F_{\Phi}(\Omega)=\left\{\sum_{j=1}^{N} \lambda_{j} \Phi\left(x_{j}, \cdot\right) \quad: \quad \lambda_{j} \in \mathbb{R}, n \in \mathbb{N}, x_{j} \in \Omega\right\}
$$

bezüglich des Skalarproduktes $(\cdot, \cdot)_{\Phi}$. Setzt man voraus, daß Punktauswertungsfunktionale in diesem Raum stetig sind, so kann man die Elemente $f$ aus $\mathcal{F}_{\Phi}(\Omega)$ mittels

$$
f(x)=\delta_{x}(f)=(f, \Phi(x, \cdot))_{\Phi}
$$

für alle $x \in \Omega$ beschreiben, und $\mathcal{F}_{\Phi}(\Omega)$ ist ein Funktionenraum. In einem solchen Fall ist ein reproduzierender Kern eine positiv definite Funktion. Da ein Hilbertraum von reellwertigen Funktionen genau dann einen reproduzierenden Kern hat, falls die zugehörigen Punktauswertungsfunktionale stetig sind, hat jede positiv definite Funktion einen Native Space.

Im Fall einer bedingt positiv definiten Basisfunktion wird zunächst der Raum der auf $\mathcal{P}$ verschwindenden Funktionale $L_{\mathcal{P}}(\Omega)$ aus (4) bezüglich der $(\cdot, \cdot)_{\Phi}$-Norm zu $\mathcal{L}_{\mathcal{P}, \Phi}(\Omega)$ vervollständigt. Da dieser Raum nicht unbedingt Funktionen enthält, konstruieren wir den Native Space mit Hilfe einer zusätzlichen, im allgemeinen aber leicht zu erfüllenden Voraussetzung. Wir nehmen an, daß $\Omega$ einen auf $\mathcal{P}$ unisolventen Punktesatz $\Xi=\left\{\xi_{1}, \ldots, \xi_{q}\right\}$ mit $q=\operatorname{dim}(\mathcal{P})$ enthält. Nun wählen wir eine Lagrangebasis $\left\{p_{1}, \ldots, p_{q}\right\}$ von $\mathcal{P}$ mit $p_{j}\left(\xi_{k}\right)=\delta_{j k}$

$$
p(x)=\sum_{j=1}^{q} p_{j}(x) p\left(\xi_{j}\right)
$$

für alle $p \in \mathcal{P}, x \in \Omega$ und definieren das Funktional

$$
\delta_{(x)}=\delta_{x}-\sum_{j=1}^{q} p_{j}(x) \delta_{\xi_{j}}
$$


aus $L_{\mathcal{P}}(\Omega)$, das Funktionen aus $\mathcal{P}$ auf die Null abbildet. Damit finden wir eine Isometrie

$$
R_{\Phi, \Omega}: \mathcal{L}_{\mathcal{P}, \Phi}(\Omega) \rightarrow \overline{R_{\Phi, \Omega}\left(L_{\mathcal{P}}(\Omega)\right)}
$$

mit $R_{\Phi, \Omega}(\lambda)(x)=\left(\lambda, \delta_{(x)}\right)_{\Phi}$ für alle $x \in \Omega$. Wegen

$$
\mu\left(R_{\Phi, \Omega}(\lambda)\right)=\left(R_{\Phi, \Omega}(\mu), R_{\Phi, \Omega}(\lambda)\right)_{\Phi}=(\mu, \lambda)_{\Phi}
$$

für alle $\mu, \lambda \in \mathcal{L}_{\mathcal{P}, \Phi}(\Omega)$ ist $R_{\Phi, \Omega}$ die Riesz-Abbildung. Addieren wir den Raum

$$
\mathcal{F}_{\Phi, \mathcal{P}}:=\overline{R_{\Phi, \Omega}\left(L_{\mathcal{P}}(\Omega)\right)}
$$

zum Nullraum $\mathcal{P}$, so erhalten wir eine direkte Summe und schreiben

Definition 2.12 Sei $\Phi$ eine bezüglich $\mathcal{P}$ bedingt positiv definite Funktion; $\Omega$ sei ein Gebiet. Dann heißt der Raum $\mathcal{N}_{\Phi, \mathcal{P}}(\Omega)=\mathcal{F}_{\Phi, \mathcal{P}}(\Omega) \oplus \mathcal{P}$ Native Space zu $\Phi$.

Wir bezeichnen nun für alle $f: \Omega \rightarrow \mathbb{R}$ den Projektor

$$
\Pi_{\mathcal{P}}(f)(x):=\sum_{j=1}^{q} p_{j}(x) f\left(\zeta_{j}\right) .
$$

Zu jedem $f \in \mathcal{N}_{\Phi, \mathcal{P}}(\Omega)$ existiert ein $\lambda_{f} \in \mathcal{L}_{\Phi, \mathcal{P}}(\Omega)$, so daß die Gleichung

$$
f-\Pi_{\mathcal{P}}(f)=R_{\Phi, \Omega}\left(\lambda_{f}\right)
$$

erfüllt ist. Daraus folgt, daß man jedes Element $f \in \mathcal{N}_{\Phi, \mathcal{P}}(\Omega)$ für alle $x \in \Omega$ mittels

$$
f(x)=\left(\Pi_{\mathcal{P}} f\right)(x)+\left(f-\Pi_{\mathcal{P}} f, R_{\Phi, \Omega}\left(\delta_{(x)}\right)\right)_{\Phi}
$$

darstellen kann. Die Funktion $\Psi$ mit $\Psi(x, y):=R_{\Phi, \Omega}\left(\delta_{(x)}\right)(y)$, die für alle $f \in \mathcal{F}_{\Phi, \mathcal{P}}(\Omega)$ die Reproduktionseigenschaft (7) erfüllt, heißt Normalisierung von $\Phi$; für sie gilt

$$
\Psi(x, y)=\left(I d-\Pi_{\mathcal{P}}\right)^{x}\left(I d-\Pi_{\mathcal{P}}\right)^{y} \Phi(x, y) .
$$

Offenbar ist $\Psi$ die reproduzierende Kernfunktion des Hilbertraumes $\mathcal{F}_{\Phi, \mathcal{P}}(\Omega)$. 


\section{Der Smolyak-Algorithmus}

Häufig wird der Smolyak-Algorithmus in der Literatur durch seinen wichtigsten Spezialfall, den Smolyak-Tensorproduktoperator, erklärt. Wir wollen diesen Begriff etwas allgemeiner verstehen und den Smolyak-Tensorproduktoperator an späterer Stelle definieren.

Wir benötigen nun zunächst $d$ Mengen $M_{1}, \ldots, M_{d}$, auf deren jeweiligen kartesischen Produkten eine Abbildung

$$
D_{l}: M_{l} \times M_{l} \rightarrow M_{l}
$$

mit $1 \leq l \leq d$ definiert sei, die wir Differenzenabbildung nennen wollen. Weiterhin sei $W$ eine Menge, welche zusammen mit einer Verknüpfung, die wir hier symbolisch als Addition oder Summation bezeichnen, einen Monoiden bildet, und es sei

$$
P: M_{1} \times \ldots \times M_{d} \rightarrow W
$$

eine Abbildung, die Produktabbildung heißen soll.

Wir definieren nun für $q, d \in \mathbb{N}, q \geq d$, den Smolyak-Algorithmus

$$
A_{q, d}:=\sum_{\substack{|j| \leq q \\ j=\left(j_{1}, \ldots, j_{d}\right) \in \mathbb{N}^{d}}} P\left(D_{1}\left(m_{j_{1}}^{1}, m_{j_{1}-1}^{1}\right), \ldots, D_{d}\left(m_{j_{d}}^{d}, m_{j_{d}-1}^{d}\right)\right)
$$

$m_{j}^{l} \in M_{l}, 1 \leq j \leq q-d+1,1 \leq l \leq d$. Der Ausdruck 'Algorithmus' kann dadurch erklärt werden, daß sein relativ kompliziertes Bildungsgesetz einer algorithmischen Anweisungsfolge nahekommt bzw. als solche verstanden werden kann.

Sinn und Zweck des Smolyak-Algorithmus kann an dieser Stelle nur sehr inexplizit beschrieben werden und wird in Verbindung mit den später aufgeführten relevanten Beispielfällen klarer verdeutlicht. Dennoch können wir ganz allgemein die Aussage treffen, daß mit Hilfe des Smolyak-Algorithmus ein multivariater Ausdruck mit im Vergleich zum mehrfachen Produkt herabgesetzter Komplexität entsteht. Das wird durch den Einsatz der bis jetzt noch nicht näher spezifizierten Differenzenabbildungen erreicht, die deshalb wesentliche Bestandteile des Smolyak-Algorithmus sind. Die Wahl der Differenzenabbildungen geschieht häufig schon implizit durch die Mengen $M_{l}$. Handelt es sich dabei etwa um Funktionenräume, so würde man als Bild der Differenzenabbildung klassische Differenzenfunktionen wählen, betrachten wir Mengen von Untervektoräumen, so wäre das Bild der Differenzenabbildung ein Differenzenvektorraum, enthalten die Mengen Operatoren, so würden wir uns für Differenzenoperatoren entscheiden, und bestünden sie aus (Daten-)Mengen, so wäre die Bildung von Differenzmengen naheliegend.

Im folgenden beschäftigen wir uns mit einer Produktabbildung, die obligatorisch wird, sobald $M_{1}, \ldots, M_{d}$ und $W$ Vektorräume sind. 


\subsection{Tensoren und Tensorprodukte}

Es gibt eine Vielzahl von Möglichkeiten, einen Zugang zu Tensoren und Tensorprodukten zu finden, vgl. etwa [1], [13], [17], [18], [20], [36]. Ganz allgemein und umfassend definiert man sie auf folgende Art und Weise:

Definition und Satz 3.1 Sind $X_{1}, \ldots, X_{d}$ Vektorräume, so gibt es einen bis auf Isomorphie eindeutig bestimmten Vektorraum $T$ zusammen mit einer multilinearen Abbildung $\otimes: X_{1} \times \ldots \times X_{d} \rightarrow T$, so daß es für alle Vektorräume $W$ und alle multilinearen Abbildungen $f: X_{1} \times \ldots \times X_{d} \rightarrow W$ genau eine lineare Abbildung $g: T \rightarrow W$ mit $f=\phi \circ g$ gibt.

Der Vektorraum $T$ wird mit $X_{1} \otimes \ldots \otimes X_{d}$ bezeichnet und heißt Tensorproduktraum oder Tensorraum von $X_{1}, \ldots, X_{d}$; seine Elemente heißen Tensoren (der Stufe d).

Der folgende Satz aus [13] ermöglicht es uns, Tensorprodukträume induktiv zu behandeln:

Satz 3.2 Seien $X_{1}, \ldots, X_{d}$ Vektorräume. Dann gilt

$$
\left(X_{1} \otimes \ldots \otimes X_{d-1}\right) \otimes X_{d} \simeq X_{1} \otimes \ldots \otimes X_{d}
$$

Wir nennen einen solchen Tensorproduktraum der Gestalt $X_{1} \otimes \ldots \otimes X_{d} d$-variat. Im folgenden reicht es häufig aus, von einer bilinearen Abbildung $\otimes$ auszugehen. Nach [13] können wir jedes Element $z$ aus $X \otimes Y$ mit den Vektorräumen $X$ und $Y$ in abstrakter Weise als

$$
z=\sum_{j=1}^{n} x_{j} \otimes y_{j}
$$

mit $x_{j} \in X, y_{j} \in Y$ darstellen; ist $\left\{\tilde{x}_{\alpha}\right\}_{\alpha \in A}$ eine Basis von $X$, so können wir $z$ als endliche Summe

$$
z=\sum_{j \in A} \tilde{x}_{j} \otimes y_{j}^{z}
$$

mit eindeutig bestimmten $y_{j}^{z} \in Y$ schreiben. Analog formulieren wir die endliche Summe

$$
z=\sum_{j \in B} x_{j}^{z} \otimes \tilde{y}_{j}
$$


falls $\left\{\tilde{y}_{\beta}\right\}_{\beta \in B}$ eine Basis von $Y$ ist. Die Elemente $x_{j}^{z}$ aus $X$ sind dabei ebenfalls eindeutig bestimmt. Die Addition zweier Elemente wird beschrieben als

$$
\sum_{j=1}^{n} x_{j} \otimes y_{j}+\sum_{j=n+1}^{m} x_{j} \otimes y_{j}=\sum_{j=1}^{m} x_{j} \otimes y_{j}
$$

falls $m>n$; Multiplikation mit $a \in \mathbb{R}$ wird mittels

$$
a \sum_{j=1}^{n} x_{j} \otimes y_{j}=\sum_{j=1}^{n} a \cdot x_{j} \otimes y_{j}=\sum_{j=1}^{n} x_{j} \otimes a \cdot y_{j}
$$

verifiziert. Eine für spätere Zwecke nötige Folgerung ist die das Nullelement betreffende Gleichung $x \otimes 0=0 \otimes y=0 \otimes 0$ für alle $x \in X$ und $y \in Y$.

Wir betrachten nun Tensorprodukträume der Form $X_{1} \otimes \ldots \otimes X_{d}$, wobei $X_{l}, 1 \leq l \leq d$, Banachräume sind. Unter Verwendung der Normen von $X_{l}$ gibt es verschiedene Möglichkeiten, vgl. [18], eine Norm auf ihrem Tensorraum zu definieren. Dabei interessieren wir uns insbesondere für solche Tensorraumnormen mit der folgenden Eigenschaft.

Definition 3.3 Eine Norm $\|\cdot\|_{X_{1} \otimes \ldots \otimes X_{d}}$ auf dem Tensorraum $X_{1} \otimes \ldots \otimes X_{d}$ der normierten Räume $\left(X_{l},\|\cdot\|_{X_{l}}\right), 1 \leq l \leq d$, heißt Kreuznorm, falls sie für alle $x_{l} \in X_{l}$ die Bedingung

$$
\left\|x_{l} \otimes \ldots \otimes x_{d}\right\|_{X_{1} \otimes \ldots \otimes X_{d}}=\left\|x_{1}\right\|_{X_{1}} \cdot \ldots \cdot\left\|x_{d}\right\|_{X_{d}}
$$

erfüllt.

Ein Beispiel für eine Kreuznorm ist die folgende Abbildung.

Definition und Lemma 3.4 Seien $\left(X_{l},\|\cdot\|_{X_{l}}\right)$ für $1 \leq l \leq d$ Banachräume. Wir bezeichnen mit $\left(X_{l}^{*},\|\cdot\|_{X_{l}^{*}}\right)$ die Dualräume von $X_{l}$, wobei

$$
\|\phi\|_{X_{l}^{*}}=\sup _{\substack{x \in X_{l} \\\|x\|_{X_{l}}=1}}|\phi(x)|
$$

definiert wird. Dann ist die Abbildung $\|\cdot\|_{\lambda, X_{1} \otimes \ldots \otimes X_{d}}: X_{1} \otimes \ldots \otimes X_{d} \rightarrow \mathbb{R}_{+}$mit

$$
\|z\|_{\lambda, X_{1} \otimes \ldots \otimes X_{d}}:=\sup _{\substack{\phi \in X_{1}^{*} \\\|\phi\|_{X_{1}^{*}=1}}}\left\|\sum_{j=1}^{n} \phi\left(x_{j}\right) y_{j}\right\|_{\lambda, X_{2} \otimes \ldots \otimes X_{d}}
$$

für $z=\sum_{j=1}^{n} x_{j} \otimes y_{j}$ und $x_{j} \in X_{1}, y_{j} \in X_{2} \otimes \ldots \otimes X_{d}$ eine Kreuznorm auf $X_{1} \otimes \ldots \otimes X_{d}$. 
Beweis: Wir zeigen die Behauptung für $d=2$ und benutzen dann Satz 3.2. Gilt $\|z\|_{\lambda, X \otimes Y}=0$, so folgern wir für alle $\phi \in X^{*}$ mit $\|\phi\|_{X^{*}}=1$

$$
\sum_{j \in B} \phi\left(x_{j}^{z}\right) \tilde{y}_{j}=0
$$

aufgrund der linearen Unabhängigkeit der $\tilde{y}_{j}$ und der Linearität von $\phi$ gilt $x_{j}^{z}=0$ für alle $j$ und damit

$$
z=\sum_{j \in B} x_{j}^{z} \otimes \tilde{y}_{j}=\sum_{j \in B} 0 \otimes \tilde{y}_{j}=0 \otimes 0 .
$$

Die Dreiecksungleichung erhalten wir aus der Linearität von $\phi$ und daraus, daß $\|\cdot\|_{Y}$ diese Eigenschaft besitzt; alle weiteren Normeigenschaften sind offensichtlich. Wir müssen nun nur noch zeigen, daß $\|\cdot\|_{\lambda, X \otimes Y}$ die Kreuznormbedingung erfüllt. Es gilt

$$
\|x \otimes y\|_{\lambda, X \otimes Y}=\sup _{\substack{\phi \in X^{*} \\\|\phi\|_{X^{*}=1}}}\|\phi(x) y\|_{Y}=\sup _{\substack{\phi \in X^{*} \\\|\phi\|_{X^{*}=1}}}|\phi(x)|\|y\|_{Y}=\|x\|_{X}\|y\|_{Y}
$$

Der Beweis folgt einer Idee aus [18]. Ähnlich definieren wir die Dualraumnorm:

Definition und Lemma 3.5 Seien $\left(X,\|\cdot\|_{X}\right)$ und $\left(Y,\|\cdot\|_{Y}\right)$ normierte Räume; $X^{*}$ und $Y^{*}$ seien ihre Dualräume. Es sei $\|\cdot\|_{\alpha, X \otimes Y}$ eine Norm auf $X \otimes Y$. Dann definiert

$$
\left\|\sum_{k=1}^{m} \phi_{k} \otimes \psi_{k}\right\|_{\alpha^{*}, X^{*} \otimes Y^{*}}=\sup _{\| \sum_{j=1}^{n} x_{j} \otimes y_{j} \in X \otimes Y}\left|\sum_{k=1}^{m} \sum_{j=1}^{n} \phi_{k}\left(x_{j}\right) \psi_{k}\left(y_{j}\right)\right|
$$

eine Norm auf $X^{*} \otimes Y^{*}$.

Wir nennen diese Tensorraumnorm die zu $\|\cdot\|_{\alpha, X \otimes Y}$ duale Norm. Es gilt:

Lemma 3.6 Die $z u\|\cdot\|_{\lambda, X \otimes Y}$ duale Norm $\|\cdot\|_{\lambda^{*}, X^{*} \otimes Y^{*}}$ ist eine Kreuznorm.

Der Beweis findet sich in [18].

Einer Tensorraumnorm nach [18], die die verschiedenen Darstellungsmöglichkeiten der Elemente aus $X_{1} \otimes \ldots \otimes X_{d}$ ausnutzt, begegnen wir im folgenden. 
Definition und Lemma 3.7 Seien $\left(X_{l},\|\cdot\|_{X_{l}}\right), 1 \leq l \leq d$, Banachräume. Dann wird durch

$$
\|z\|_{\gamma, X_{1} \otimes \ldots \otimes X_{d}}=\inf _{\substack{z=\sum_{j=1}^{n} x_{j} \otimes y_{j} \\ x_{j} \in X_{1}, y_{j} \in X_{2} \otimes \ldots \otimes X_{d}}} \sum_{j=1}^{n}\left\|x_{j}\right\|_{X_{1}}\left\|y_{j}\right\|_{\gamma, X_{2} \otimes \ldots \otimes X_{d}}
$$

eine Kreuznorm definiert.

Bemerkung 3.8 Die besondere Eigenschaft der $\lambda$-und $\gamma$-Kreuznormen besteht darin, daß sie für jede Kreuznorm $\|\cdot\|_{\alpha, X_{1} \otimes \ldots \otimes X_{d}}$ und für jedes $z \in X_{1} \otimes \ldots \otimes X_{d}$ die Ungleichung

$$
\|z\|_{\lambda, X_{1} \otimes \ldots \otimes X_{d}} \leq\|z\|_{\alpha, X_{1} \otimes \ldots \otimes X_{d}} \leq\|z\|_{\gamma, X_{1} \otimes \ldots \otimes X_{d}}
$$

erfüllen. Der Beweis findet sich bei Schatten [36].

Die folgende Tensorraumnorm stellt eine Erweiterung der $\gamma$-Kreuznorm dar:

Definition und Lemma 3.9 Für einen Banachraum $\left(X_{l},\|\cdot\|_{X_{l}}\right), 1 \leq l \leq d$, sei die $p$-Nuklearnorm für $1 \leq p \leq \infty$ mit $\frac{1}{p}+\frac{1}{q}=1$ und $z \in X_{1} \otimes \ldots \otimes X_{d}$ definiert als

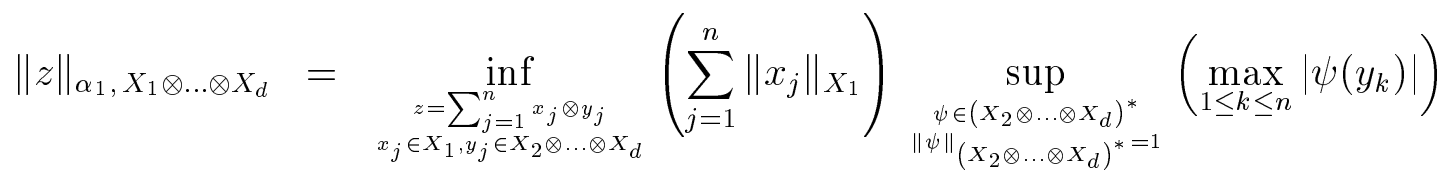

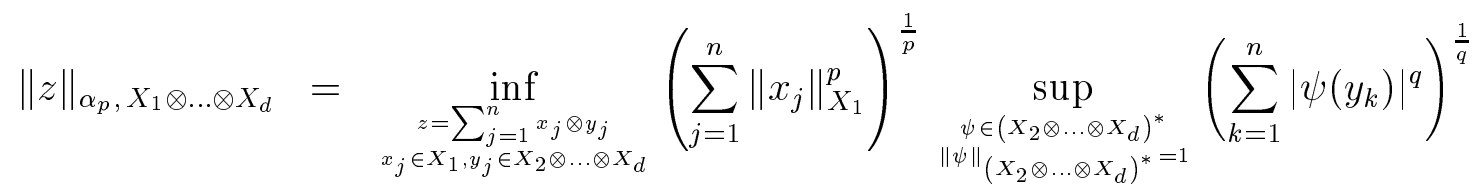

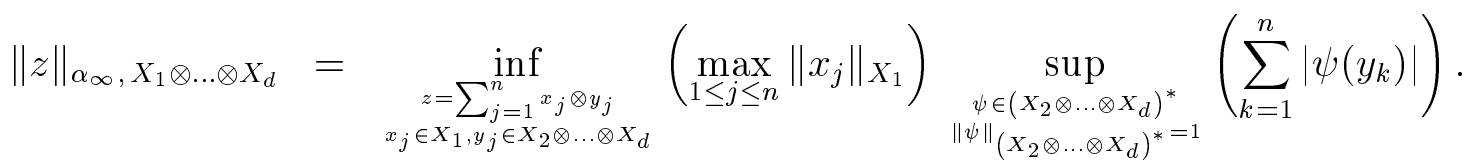

Die p-Nuklearnorm ist eine Kreuznorm.

Den Beweis finden wir bei [18]. Mit Bemerkung 3.8 wird offensichtlich, daß die $\gamma$-Tensorraumnorm und die $\alpha_{1}$-Tensorraumnorm übereinstimmen.

Eine weitere Eigenschaft von Kreuznormen spezifizieren wir mit Hilfe der folgenden 
Definition 3.10 Seien $A_{l}: X_{l} \rightarrow V_{l}, 1 \leq l \leq d$, lineare Operatoren, wobei $X_{l}$ und $V_{l}$ Vektorräume darstellen. Dann heißt der Operator $A_{1} \otimes \ldots \otimes A_{d}: X_{1} \otimes \ldots \otimes X_{d} \rightarrow V_{1} \otimes \ldots \otimes V_{d}$ mit

$$
\left(A_{1} \otimes \ldots \otimes A_{d}\right) z=\sum_{j=1}^{n}\left(A_{1} x_{j}\right) \otimes\left(\left(A_{2} \otimes \ldots \otimes A_{d}\right) y_{j}\right)
$$

und $z=\sum_{j=1}^{n} x_{j} \otimes y_{j}$ für alle $x_{j} \in X_{1}, y_{j} \in X_{2} \otimes \ldots \otimes X_{d}$ Tensorproduktoperator.

Wählen wir die nicht-rekursive Darstellung des Tensorproduktoperators, so schreiben wir

$$
\left(A_{1} \otimes \ldots \otimes A_{d}\right) z=\sum_{\substack{1 \leq j_{j} \leq n_{l} \\ 1 \leq l \leq d-1}}\left(A_{1} x_{1 j_{1}}\right)\left(A_{2} x_{2 j_{1} j_{2}}\right) \cdot \ldots \cdot\left(A_{d-1} x_{d-1 j_{1} \ldots j_{d-1}}\right)\left(A_{d} x_{d j_{1} \ldots j_{d-1}}\right)
$$

für alle $z=\sum_{\substack{1 \leq j_{l} \leq n_{l} \\ 1 \leq l \leq d}} x_{1 j_{1}} x_{2 j_{1} j_{2}} \otimes \ldots \otimes x_{d-1 j_{1} \ldots j_{d-1}} \otimes x_{d j_{1} \ldots j_{d-1}}$ mit $x_{l j_{1} \ldots j_{l}} \in X_{l}$, $1 \leq l \leq d-1$, und $x_{d j_{1} \ldots j_{d-1}} \in X_{d}$.

Da wir lineare Operatoren betrachten, folgern wir die Unabhängigkeit von der Darstellung von $z$, wenn wir die Basen der Räume $X_{l}$ benutzen. Nun formulieren wir:

Definition 3.11 Seien $\left(X_{l},\|\cdot\|_{X_{l}}\right)$ und $\left(V_{l},\|\cdot\|_{V_{l}}\right)$ für $1 \leq l \leq d$ Banachräume. Die Tensorraumnorm $\|\cdot\|_{X_{1} \otimes \ldots \otimes X_{d}}$ heißt mit der Tensorraumnorm $\|\cdot\|_{V_{1} \otimes \ldots \otimes V_{d}}$ gleichmäßig verträglich, falls sie die Ungleichung

$$
\left\|\left(A_{1} \otimes \ldots \otimes A_{d}\right) z\right\|_{V_{1} \otimes \ldots \otimes V_{d}} \leq\left\|A_{1}\right\|_{X_{1}, V_{1}} \cdot \ldots \cdot\left\|A_{d}\right\|_{X_{d}, V_{d}}\|z\|_{X_{1} \otimes \ldots \otimes X_{d}}
$$

für alle linearen Operatoren $A_{l}: X_{l} \rightarrow V_{l}, 1 \leq l \leq d$, und für alle $z \in X_{1} \otimes \ldots \otimes X_{d}$ mit

$$
\left\|A_{l}\right\|_{X_{l}, V_{l}}=\sup _{\substack{x \in X_{l} \\\|x\|_{X_{l}=1}=1}}\left\|A_{l} x\right\|_{V_{l}}
$$

erfüllt. Wir nennen eine Tensorproduktraumnorm $\|\cdot\|_{X_{1} \otimes \ldots \otimes X_{d}}$ im Fall $X_{l}=V_{l}$, $1 \leq l \leq d$, gleichmäßig, falls sie mit sich selbst gleichmäßig verträglich ist.

Definieren wir

$$
\|A \otimes B\|_{X_{1} \otimes \ldots \otimes X_{d}, V_{1} \otimes \ldots \otimes V_{d}}:=\sup _{\substack{z \in X_{1} \otimes \ldots \otimes X_{d} \\\|z\| X_{1} \otimes \ldots \otimes X_{d}=1}}\left\|\left(A_{1} \otimes \ldots \otimes A_{d}\right) z\right\|_{V_{1} \otimes \ldots \otimes V_{d}}
$$


so gilt wegen

$$
\begin{aligned}
& \left\|A_{1} \otimes \ldots \otimes A_{d}\right\|_{X_{1} \otimes \ldots \otimes X_{d}, V_{1} \otimes \ldots \otimes V_{d}} \\
& \quad \geq \quad \sup _{\substack{x_{1} \otimes \ldots \otimes x_{d} \in X_{1} \otimes \ldots \otimes X_{d} \\
\left\|x_{1} \otimes \ldots \otimes x_{d}\right\| X_{1} \otimes \ldots \otimes X_{d}=1}}\left\|\left(A_{1} \otimes \ldots \otimes A_{d}\right)\left(x_{1} \otimes \ldots \otimes x_{d}\right)\right\|_{V_{1} \otimes \ldots \otimes V_{d}} \\
& =\sup _{\substack{x_{l} \in X_{l} \\
\left\|x_{l}\right\| X_{l}=1 \\
1 \leq l \leq d}}\left\|\left(A_{1} x_{1}\right) \otimes \ldots \otimes\left(A_{d} x_{d}\right)\right\|_{V_{1} \otimes \ldots \otimes V_{d}} \\
& =\sup _{\substack{x_{l} \in X_{l} \\
\left\|x_{l}\right\| X_{l}=1 \\
1 \leq l \leq d}}\left\|A_{1} x_{1}\right\|_{V_{1}} \cdot \ldots \cdot\left\|A_{d} x_{d}\right\|_{V_{d}} \\
& =\left\|A_{1}\right\| X_{1}, V_{1} \cdot \ldots \cdot\left\|A_{d}\right\|_{X_{d}, V_{d}}
\end{aligned}
$$

für die auf gleichmäßig verträglichen Kreuznormen basierenden Operatornormen die Gleichung

$$
\left\|A_{1} \otimes \ldots \otimes A_{d}\right\|_{X_{1} \otimes \ldots \otimes X_{d}, V_{1} \otimes \ldots \otimes V_{d}}=\left\|A_{1}\right\|_{X_{1}, V_{1}} \cdot \ldots \cdot\left\|A_{d}\right\|_{X_{d}, V_{d}} .
$$

Den Abschluß eines Tensorproduktraumes $X_{1} \otimes \ldots \otimes X_{d}$ bezüglich einer Tensorraumnorm $\|\cdot\|_{\alpha, X_{1} \otimes \ldots \otimes X_{d}}$ bezeichnen wir mit $X_{1} \otimes_{\alpha} \ldots \otimes_{\alpha} X_{d}$. Ist die Kreuznorm $\|\cdot\|_{\alpha_{X}, X_{1} \otimes \ldots \otimes X_{d}}$ mit der Kreuznorm $\|\cdot\|_{\alpha_{V}, V_{1} \otimes \ldots \otimes V_{d}}$ gleichmäßig verträglich, so gilt

$$
\left\|A_{1} \otimes \ldots \otimes A_{d}\right\|_{X_{1} \otimes_{\alpha_{X}} \cdots \otimes_{\alpha_{X}} X_{d}, V_{1} \otimes_{\alpha_{V}} \ldots \otimes_{\alpha_{V}} V_{d}}=\left\|A_{1}\right\|_{X_{1}, V_{1}} \cdot \ldots \cdot\left\|A_{d}\right\|_{X_{d}, V_{d}}
$$

für alle linearen Abbildungen $A_{l}: X_{l} \rightarrow V_{l}, 1 \leq l \leq d$. Da diese Eigenschaft (8) für die kommenden Ausführungen von entscheidender Bedeutung sein wird, wollen wir im folgenden alle zu normierten Tensorräumen bzw. zu deren Abschlüssen isomorphen Räume als Tensorräume bezeichnen.

Beispiele für verträgliche bzw. gleichmäßig verträgliche Tensorraumnormen sind die bekannten Normen:

Satz 3.12 Für die Banachräume $X_{l}$ und $V_{l}, 1 \leq l \leq d$, mit zugehöriger Norm gilt

(a) $\|\cdot\|_{\lambda, X_{1} \otimes \ldots \otimes X_{d}}$ und $\|\cdot\|_{\lambda, V_{1} \otimes \ldots \otimes V_{d}}$ sind gleichmäßig verträglich;

(b) Jede Kreuznorm $\|\cdot\|_{\alpha, X_{1} \otimes \ldots \otimes X_{d}}$ ist mit $\|\cdot\|_{\lambda, V_{1} \otimes \ldots \otimes V_{d}}$ gleichmäßig verträglich;

(c) $\|\cdot\|_{\gamma, X_{1} \otimes \ldots \otimes X_{d}}$ und $\|\cdot\|_{\gamma, V_{1} \otimes \ldots \otimes V_{d}}$ sind gleichmäßig verträglich;

(d) $\|\cdot\|_{\gamma, X_{1} \otimes \ldots \otimes X_{d}}$ ist mit jeder Kreuznorm $\|\cdot\|_{\alpha, V_{1} \otimes \ldots \otimes V_{d}}$ gleichmäßig verträglich;

(e) $\|\cdot\|_{\alpha_{p}, X_{1} \otimes \ldots \otimes X_{d}}$ und $\|\cdot\|_{\alpha_{p}, V_{1} \otimes \ldots \otimes V_{d}}$ sind für $1 \leq p \leq \infty$ gleichmäßig verträglich. 
Beweis: Auch hier reicht es aus, die Behauptung für $d=2$ zu zeigen. Wir bezeichnen $A_{1}=A, A_{2}=B, X_{1}=X, X_{2}=Y, V_{1}=V$ und $V_{2}=W$. Es gilt bei (a) unter Verwendung von Definition und Kreuznormeigenschaft der dualen Norm

$$
\begin{aligned}
& \left\|(A \otimes B) \sum_{j=1}^{n} x_{j} \otimes y_{j}\right\|_{\lambda, V \otimes W} \\
& =\sup _{\substack{\phi \in V^{*} \\
\|\phi\|_{V^{*}=1}}}\left\|\sum_{j=1}^{n} \phi\left(A x_{j}\right) \otimes\left(B y_{j}\right)\right\|_{W} \\
& =\sup _{\substack{\phi \in V^{*} \\
\|\phi\|_{V^{*}=1}}} \sup _{\substack{\psi \in W^{*} \\
\|\psi\|_{W^{*}=1}}}\left|\sum_{j=1}^{n} \phi\left(A x_{j}\right) \otimes \psi\left(B y_{j}\right)\right| \\
& =\sup _{\substack{\phi \in V^{*} \\
\|\phi\|_{V^{*}=1}}} \sup _{\substack{\psi \in W^{*} \\
\|\psi\|_{W^{*}=1}}}((\phi \circ A) \otimes(\psi \circ B))\left|\sum_{j=1}^{n} x_{j} \otimes y_{j}\right| \\
& \leq \sup _{\substack{\phi \in V^{*} \\
\|\phi\|_{V^{*}=1}}} \sup _{\substack{\psi \in W^{*} \\
\|\|_{W^{*}=1}}}\|(\phi \circ A) \otimes(\psi \circ B)\|_{\lambda^{*}, X^{*} \otimes Y^{*}}\left\|\sum_{j=1}^{n} x_{j} \otimes y_{j}\right\|_{\lambda, X \otimes Y} \\
& =\sup _{\substack{\phi \in V^{*} \\
\|\phi\|_{V^{*}=1}}} \sup _{\substack{\psi \in W^{*} \\
\|\psi\|_{W^{*}=1}}}\|\phi \circ A\|_{X^{*}}\|\psi \circ B\|_{Y^{*}}\left\|\sum_{j=1}^{n} x_{j} \otimes y_{j}\right\|_{\lambda, X \otimes Y} \\
& =\sup _{\substack{\phi \in V^{*} \\
\|\phi\|_{V^{*}=1}}} \sup _{\substack{x \in X \\
\|x\|_{X}=1}}|\phi(A x)| \sup _{\substack{\psi \in W^{*} \\
\|\psi\|_{W^{*}=1}}} \sup _{\substack{y \in Y \\
\|y\|_{Y}=1}}|\psi(B y)|\left\|\sum_{j=1}^{n} x_{j} \otimes y_{j}\right\|_{\lambda, X \otimes Y} \\
& =\|A\|_{X, V}\|B\|_{Y, W}\left\|\sum_{j=1}^{n} x_{j} \otimes y_{j}\right\|_{\lambda, X \otimes Y} .
\end{aligned}
$$

Der Fall (c) ist ein Spezialfall von Fall (e). Für (e) gilt einerseits

$$
\left(\sum_{j=1}^{n}\left\|A x_{j}\right\|_{V}^{p}\right)^{\frac{1}{p}} \leq\|A\|_{X, V}\left(\sum_{j=1}^{n}\left\|x_{j}\right\|_{X}^{p}\right)^{\frac{1}{p}}
$$

und genauso

$$
\max _{1 \leq j \leq n}\left\|A x_{j}\right\|_{V} \leq\|A\|_{X, V} \max _{1 \leq j \leq n}\left\|x_{j}\right\|_{X}
$$

andererseits

$$
\sup _{\substack{\psi \in V^{*} \\\|\psi\|_{V^{*}=1}}}\left(\sum_{k=1}^{n}\left|\psi\left(B y_{k}\right)\right|^{q}\right)^{\frac{1}{q}} \leq \sup _{\substack{\psi \\\|\|_{V^{*}} \\\|\|_{V^{*}=1}}}\left(\sum_{k=1}^{n}\|\psi\|_{V^{*}}^{q}\left\|B y_{k}\right\|_{W^{*}}^{q}\right)^{\frac{1}{q}}
$$




$$
\begin{aligned}
& \leq\|B\|_{Y, W}\left(\sum_{k=1}^{n}\left\|y_{k}\right\|_{Y}^{q}\right)^{\frac{1}{q}} \\
& =\|B\|_{Y, W} \sup _{\substack{\psi \in V^{*} \\
\|\psi\|_{V^{*}=1}}}\left(\sum_{k=1}^{n}\left|\psi\left(y_{k}\right)\right|^{q}\right)^{\frac{1}{q}}
\end{aligned}
$$

und mit analogen Schritten

$$
\sup _{\substack{\psi \in V^{*} \\\|\psi\|_{V^{*}=1}}} \max _{1 \leq k \leq n}\left|\psi\left(B y_{k}\right)\right| \leq\|B\|_{Y, W} \sup _{\substack{\psi \in V^{*} \\\|\psi\|_{V^{*}=1}}} \max _{1 \leq k \leq n}\left|\psi\left(y_{k}\right)\right| .
$$

Insgesamt folgern wir in den Fällen (a), (c) und (e) durch beidseitiges Anwenden des Infimums inf $\sec _{z=\sum_{\substack{j=1 \\ x_{j} \in X, y_{j} \in Y}}^{n} x_{j} \otimes y_{j}}$ die Behauptungen. Die Fälle (b) und (d) folgen aus (a) und (c) mit Bemerkung 3.8.

Der folgende Satz aus [18] und [21] führt zu einer weiteren Kreuznorm.

Satz 3.13 Seien $H_{1}, H_{2}$ reelle Hilberträume mit Skalarprodukten $(\cdot, \cdot)_{H_{1}}$ und $(\cdot, \cdot)_{H_{2}}$. Dann wird für die Elemente $h=\sum_{j=1}^{n} h_{1 j} \otimes h_{2 j}$ bzw. $\tilde{h}=\sum_{k=1}^{m} \tilde{h}_{1 k} \otimes \tilde{h}_{2 k}$ aus dem Tensorproduktraum $\mathrm{H}_{1} \otimes \mathrm{H}_{2}$ mittels

$$
(h, \tilde{h})_{\beta, H_{1} \otimes H_{2}}:=\sum_{j=1}^{n} \sum_{k=1}^{m}\left(h_{1 j}, \tilde{h}_{1 k}\right)_{H_{1}}\left(h_{2 j}, \tilde{h}_{2 k}\right)_{H_{2}}
$$

ein Skalarprodukt auf $H_{1} \otimes H_{2}$ definiert.

Analoges gilt aufgrund von Satz 3.2 für mehrfache Tensorprodukte von Hilberträumen. Weiterhin gilt:

Lemma 3.14 Die Skalarprodukte $(\cdot, \cdot)_{\beta, H_{1} \otimes \ldots \otimes H_{d}}$ bzw. $(\cdot, \cdot)_{\beta, \tilde{H}_{1} \otimes \ldots \otimes \tilde{H}_{d}}$ auf den Tensorprodukten von Hilberträumen $H_{1}, \ldots, H_{d}$ bzw. $\tilde{H}_{1}, \ldots, \tilde{H}_{d}$ induzieren gleichmäßig verträgliche Kreuznormen $\|\cdot\|_{\beta, H_{1} \otimes \ldots \otimes H_{d}}$ bzw. $\|\cdot\|_{\beta, \tilde{H}_{1} \otimes \ldots \otimes \tilde{H}_{d}}$.Der Abschluß $H_{1} \otimes_{\beta} \ldots \otimes_{\beta} H_{d}$ bzgl. $\|\cdot\|_{\beta, H_{1} \otimes \ldots \otimes H_{d}}$ ist ein Hilbertraum.

Der Beweis folgt im wesentlichen [18] und [21].

Wir sind nun in der Lage, den Smolyak-Tensorproduktoperator zu definieren. 


\subsection{Bildung und Eigenschaften des Smolyak-Tensorprodukt- Algorithmus}

Der Smolyak-Tensorproduktoperator besteht aus einer auf eine bestimmte Weise gebildeten Linearkombination von Tensorproduktoperatoren. Wir benötigen dazu vektorräume $\mathcal{F}$ und $\mathcal{V}$, univariate lineare Operatoren $U_{1}, U_{2}, \ldots$ mit $U_{j}: \mathcal{F}_{j} \rightarrow \mathcal{V}_{j}$, wobei $\mathcal{F}_{j} \subseteq \mathcal{F}$ und $\mathcal{V}_{j} \subseteq \mathcal{V}$ Vektorräume bezeichnen und zudem die Differenzenoperatoren $\Delta_{j}:=U_{j}-U_{j-1}, j \in N_{>1}$, bzw. $\Delta_{1}:=U_{1}$, welche die Vektorräume $\mathcal{F}_{j}-\mathcal{F}_{j-1}$ auf die Vektorräume $\mathcal{V}_{j}-\mathcal{V}_{j-1}$ abbilden. Wir definieren nun für alle $q \geq d \in \mathbb{N}$ den Smolyak-Tensorproduktoperator

$$
A_{q, d}:=\sum_{\substack{|j| \leq q \\ j=\left(j_{1}, \ldots, j_{d}\right) \in \mathbb{N}^{d}}}\left(\Delta_{j_{1}} \otimes \ldots \otimes \Delta_{j_{d}}\right) ;
$$

er bildet Elemente des Vektorraumes $\mathcal{S V}_{q, d}^{\mathcal{F}_{1}, \ldots, \mathcal{F}_{q-d+1}}$ auf den Vektorraum $\mathcal{S V}_{q, d}^{\mathcal{V}_{1}, \ldots, \mathcal{V}_{q-d+1}}$ ab, wobei wir den Smolyakraum

$$
\mathcal{S}_{\mathcal{X}^{\prime}, d}^{\mathcal{X}_{1}, \ldots, \mathcal{X}_{q-d+1}}:=\sum_{\substack{|j| \leq q \\ j=\left(j_{1}, \ldots, j_{d}\right) \in \mathbb{N}^{d}}}\left(\left(\mathcal{X}_{j_{1}}-\mathcal{X}_{j_{1}-1}\right) \otimes \ldots \otimes\left(\mathcal{X}_{j_{d}}-\mathcal{X}_{j_{d}-1}\right)\right)
$$

unter Verwendung der Vektorräume $\mathcal{X}_{1}, \ldots, \mathcal{X}_{q-d+1}$ bezeichnen. Im Fall $\mathcal{X}_{1}=\ldots=\mathcal{X}_{q-d+1}=: \mathcal{X}$ gilt natürlich

$$
\mathcal{S V}_{q, d}^{\mathcal{X}_{1}, \ldots, \mathcal{X}_{q-d+1}}=\mathcal{X} \otimes \ldots \otimes \mathcal{X}
$$

Sind $\left(\mathcal{F},\|\cdot\|_{\mathcal{F}}\right)$ und $\left(\mathcal{V},\|\cdot\|_{\mathcal{V}}\right)$ normierte Vektorräume und gilt $\mathcal{F}_{j} \subseteq \mathcal{F}$ bzw. $\mathcal{V}_{j} \subseteq \mathcal{V}$, so werden auf $\mathcal{S V}_{q, d}^{\mathcal{F}_{1}, \ldots, \mathcal{F}_{q-d+1}}$ und $\mathcal{S V}_{q, d}^{\mathcal{V}_{1}, \ldots, \mathcal{V}_{q-d+1}}$ durch Nutzung der Tensorraumnormen $\|\cdot\|_{\alpha_{\mathcal{F}},\left(\mathcal{F}_{j_{1}}-\mathcal{F}_{j_{1}-1}\right) \otimes \ldots \otimes\left(\mathcal{F}_{j_{d}}-\mathcal{F}_{j_{d}-1}\right)}$ bzw. $\|\cdot\|_{\alpha_{\mathcal{V}},\left(\mathcal{V}_{j_{1}}-\mathcal{V}_{j_{1}-1}\right) \otimes \ldots \otimes\left(\mathcal{V}_{j_{d}}-\mathcal{V}_{j_{d}-1}\right)}$ zwei Normen definiert, die auf den Unterräumen $\mathcal{S V}_{q, d}^{\mathcal{F}_{1}, \ldots, \mathcal{F}_{q-d+1}}$ bzw. $\mathcal{S V}_{q, d}^{\mathcal{V}_{1}, \ldots, \mathcal{V}_{q-d+1}}$ der Tensorräume $\mathcal{F} \otimes_{\alpha_{\mathcal{F}}} \ldots \otimes_{\alpha_{\mathcal{F}}} \mathcal{F}$ bzw. $\mathcal{V} \otimes_{\alpha_{\mathcal{V}}} \ldots \otimes_{\alpha_{\mathcal{V}}} \mathcal{V}$ mit den Tensorraumnormen übereinstimmen.

Offensichtlich erhalten wir im trivialen Fall $A_{d, d}=\mathbf{U}_{d, d}=U_{1} \otimes \ldots \otimes U_{1}$. Weiterhin gilt $A_{q, 1}=U_{q}$ im Eindimensionalen und

$$
A_{q, 2}=\sum_{j=1}^{q-1}\left(U_{j} \otimes U_{q-j}\right)-\sum_{j=1}^{q-2}\left(U_{j} \otimes U_{q-1-j}\right)
$$

für $d=2$. Eine andere, für praktische Belange hilfreiche Formulierung des SmolyakTensorproduktoperators geht auf Wasilkowski und Woźniakowski [46] zurück. Wir definieren dazu den $d$-variaten Tensorproduktoperator

$$
\mathbf{U}_{j, d}:=U_{j_{1}} \otimes \ldots \otimes U_{j_{d}}
$$

mit $j=\left(j_{1}, \ldots, j_{d}\right) \in \mathbb{N}^{d}$; es gilt 
Satz 3.15 Der Smolyak-Tensorproduktoperator erfüllt die Identität

$$
A_{q, d}=\sum_{q-d+1 \leq|j|_{d} \leq q}(-1)^{q-|j|_{d}}\left(\begin{array}{c}
d-1 \\
q-|j|_{d}
\end{array}\right) \mathbf{U}_{j, d} .
$$

Wir bezeichnen dabei im folgenden immer $|j|_{d}=|j|$, falls $j=\left(j_{1}, \ldots, j_{d}\right) \in \mathbb{N}_{d}$ gilt.

Für die spätere Anwendung benötigen wir:

Lemma 3.16 Für den Smolyak-Tensorproduktoperator gilt

$$
\text { (a) } \begin{aligned}
A_{q, d} & =\sum_{l=d-1}^{q-1} A_{l, d-1} \otimes \Delta_{q-l}=\sum_{l=d-1}^{q-1} \Delta_{q-l} \otimes A_{l, d-1} ; \\
\text { (b) } A_{q, d} & =\sum_{|j|_{d-1} \leq q-1} \Delta_{j_{1}} \otimes \ldots \otimes \Delta_{j_{d-1}} \otimes U_{q-|j|_{d-1}} \\
& =\sum_{|j|_{d-1} \leq q-1} U_{q-|j|_{d-1}} \otimes \Delta_{j_{1}} \otimes \ldots \otimes \Delta_{j_{d-1}} .
\end{aligned}
$$

Beweis: Es gilt bei (a)

$$
\begin{aligned}
A_{q, d} & =\sum_{|i|_{d} \leq q} \Delta_{i_{1}} \otimes \ldots \otimes \Delta_{i_{d}} \\
& =\sum_{j=d}^{q} \sum_{l=1}^{j-d+1} \sum_{\mid i_{d-1}=j-l} \Delta_{i_{1}} \otimes \ldots \otimes \Delta_{i_{d-1}} \otimes \Delta_{l} \\
& =\sum_{l=d-1}^{q-1} \sum_{\mid i_{\left.\right|_{d-1}}}^{l} \Delta_{i_{1}} \otimes \ldots \otimes \Delta_{i_{d-1}} \otimes \Delta_{q-l} .
\end{aligned}
$$

Im Fall (b) folgern wir

$$
\begin{aligned}
A_{q, d} & =\sum_{l=d-1}^{q-1}\left(A_{l, d-1} \otimes \Delta_{q-l}\right) \\
& =\sum_{l=d-1}^{q-1}\left(A_{l, d-1} \otimes U_{q-l}\right) \sum_{l=d-2}^{q-1}\left(A_{l-1, d-1} \otimes U_{q-l}\right) \\
& =A_{d-1, d-1} \otimes U_{q-d+1}+\sum_{l=d-2}^{q-1} \sum_{|j|_{d-1}=l} \Delta_{j_{1}} \otimes \ldots \otimes \Delta_{j_{d-1}} \otimes U_{q-l} \\
& =\sum_{l=d-1}^{q-1} \sum_{|j|_{d-1}=l} \Delta_{j_{1}} \otimes \ldots \otimes \Delta_{j_{d-1}} \otimes U_{q-l} ;
\end{aligned}
$$


daraus folgt die erste Gleichheit; die zweite läßt sich für (a) und (b) völlig analog beweisen.

Häufig verwandt wird der Smolyak-Tensorprodukt-Algorithmus unter Nutzung von Operatoren $U_{j}: \mathcal{F}_{j} \rightarrow \mathcal{V}_{j}, 1 \leq j \leq q-d+1$, der Form

$$
U_{j}(f):=\sum_{l=1}^{m_{j}} a_{j l} f\left(x_{j l}\right)=\sum_{l=1}^{m_{j}} a_{j l} \delta_{x_{j l}}(f),
$$

wobei $\mathcal{F}_{j}$ und $\mathcal{V}_{j} \mathbb{R}$-Vektorräume sind und $f \in \mathcal{F}_{j}$ gilt. Die Daten $x_{j l} \in \mathbb{R}$, ihre Anzahl $m_{j} \in \mathbb{I N}$ und $a_{j l} \in \mathcal{V}_{j}$ werden dabei für alle Werte von $l$ und $j$ als gegeben vorausgesetzt. Weiterhin bezeichne $\delta$ das Punktauswertungsfunktional; es gelte $\delta_{x}(f)=f(x)$ für einen festen Punkt $x \in \mathbb{R}$ und eine Funktion $f$. Das Tensorprodukt zweier solcher Operatoren $U_{\alpha}$ und $U_{\beta}, 1 \leq \alpha, \beta \leq q-d+1$, angewandt auf das Element $f=\sum_{k=1}^{n} f_{\alpha, k} \otimes f_{\beta, k}$ eines Tensorraumes $\mathcal{F}_{\alpha} \otimes \mathcal{F}_{\beta}$, erhält dann nach Definition 3.10 die Gestalt

$$
\begin{aligned}
\left(U_{\alpha} \otimes U_{\beta}\right)\left(\sum_{k=1}^{n} f_{\alpha, k} \otimes f_{\beta, k}\right) & =\sum_{k=1}^{n} \sum_{l_{\alpha}=1}^{m_{\alpha}} f_{\alpha, k}\left(x_{\alpha l_{\alpha}}\right) \sum_{l_{\beta}=1}^{m_{\beta}} f_{\beta, k}\left(x_{\beta l_{\beta}}\right) a_{\alpha l_{\alpha}} \otimes a_{\beta l_{\beta}} \\
& =\sum_{l_{\alpha}=1}^{m_{\alpha}} \sum_{l_{\beta}=1}^{m_{\beta}} \sum_{k=1}^{n} f_{\alpha, k} \otimes f_{\beta, k}\left(x_{\alpha l_{\alpha}}, x_{\beta l_{\beta}}\right) a_{\alpha l_{\alpha}} \otimes a_{\beta l_{\beta}} \\
& =\sum_{l_{\alpha}=1}^{m_{\alpha}} \sum_{l_{\beta}=1}^{m_{\beta}} f\left(x_{\alpha l_{\alpha}}, x_{\beta l_{\beta}}\right) a_{\alpha l_{\alpha}} \otimes a_{\beta l_{\beta}} .
\end{aligned}
$$

Für die Tensorproduktoperatoren $\mathbf{U}_{j}: \mathcal{F}_{j_{1}} \otimes \ldots \otimes \mathcal{F}_{j_{d}} \rightarrow \mathcal{V}_{j_{1}} \otimes \ldots \otimes \mathcal{V}_{j_{d}}$ folgt induktiv mit $j=\left(j_{1}, \ldots, j_{d}\right)$

$$
\begin{aligned}
\mathbf{U}_{j}(f) & =\left(U_{i_{1}} \otimes \ldots \otimes U_{i_{d}}\right)(f) \\
& =\left(\sum_{l_{1}=1}^{m_{j_{1}}} a_{j_{1} l_{1}} \delta_{x_{j_{1} l_{1}}} \otimes \ldots \otimes \sum_{l_{d}=1}^{m_{j_{d}}} a_{j_{d} l_{d}} \delta_{x_{j_{d} l_{d}}}\right)(f) \\
& =\sum_{\substack{1 \leq k_{l} \leq m_{j_{l}} \\
1 \leq l \leq d}} f\left(x_{j_{1} k_{1}}, \ldots, x_{j_{d} k_{d}}\right)\left(a_{j_{1} k_{1}} \otimes \ldots \otimes a_{j_{d} k_{d}}\right)
\end{aligned}
$$

und der Smolyak-Tensorproduktoperator bekommt für $f \in \mathcal{S V}_{q, d}^{\mathcal{F}_{1}, \otimes, \mathcal{F}_{q-d+1}}$ die Gestalt

$$
\begin{aligned}
& A_{q, d}(f) \\
& =\sum_{q-d+1 \leq|j|_{d} \leq q}(-1)^{q-|j|_{d}}\left(\begin{array}{c}
d-1 \\
q-|j|_{d}
\end{array}\right) \mathbf{U}_{j}
\end{aligned}
$$




$$
=\sum_{\substack{q-d+1 \leq|j|_{d} \leq q \\
1 \leq k_{l} \leq m_{j} \\
k=\left(k_{1}, \ldots, k_{d}\right) \in \mathbb{N}^{d}}}(-1)^{q-|j|_{d}}\left(\begin{array}{c}
d-1 \\
q-|j|_{d}
\end{array}\right) f\left(x_{j_{1} k_{1}}, \ldots, x_{j_{d} k_{d}}\right)\left(a_{j_{1} k_{1}} \otimes \ldots \otimes a_{j_{d} k_{d}}\right) .
$$

In einigen Fällen wie etwa der numerischen Integration, vgl. [25], nimmt man $\mathcal{V}_{j}=\mathbb{R}$ für alle Werte von $j$ an, im Rahmen der Interpolations- und Approximationstheorie fällt die Wahl naturgemäß auf Funktionenräume.

Im folgenden Abschnitt soll die Struktur der vom Smolyak-Tensorproduktoperator (9) verwandten Daten $\left\{x_{j l}\right\}_{j, l}$ näher betrachtet werden. Dabei wird deutlich werden, daß auch sie dem Bildungsgesetz des Smolyak-Algorithmus folgen.

\subsection{Das dünne Gitter und die Anzahl seiner Punkte}

Basis für die Anwendung des Smolyak-Tensorproduktoperators bildet in vielen Fällen ein entsprechendes $d$-dimensionales sogenanntes dünnes Gitter, vgl. (9). Diese Bezeichnung ist darin begründet, daß das dünne Gitter weniger Knotenpunkte enthält als im klassischen Fall und von daher mit zunehmender Dimension auch keinen so starken Anstieg seiner Gitterpunktanzahl zu verzeichnen hat. Genauer definieren wir das dünne Gitter zunächst als

$$
H_{q, d}:=\bigcup_{|k|_{d} \leq q}\left(X_{k_{1}} \times \ldots \times X_{k_{d}}\right)
$$

mit $d, q \in I N, q \geq d$, und den reellen Punktmengen

$$
X_{j}:=\left\{x_{j 1}, \ldots, x_{j m_{j}}\right\} .
$$

Indem wir diese Mengen $X_{j}, 1 \leq j \leq q-d+1$, sukzessive ineinander verschachteln und ihre Elemente entsprechend ordnen, d.h. $X_{1} \subset X_{2} \subset \ldots \subset X_{q-d+1}$, wobei $x_{j i}=x_{k i}$ erfüllt ist, falls $j \leq k$ und $i \leq m_{i}$ gilt, fordern wir damit einerseits $m_{j} \leq m_{k}$ für $j \leq k$ und andererseits erhalten wir die vereinfachte Darstellung

$$
H_{q, d}=\bigcup_{|k|_{d}=q}\left(X_{k_{1}} \times \ldots \times X_{k_{d}}\right)
$$

Unter diesen Umständen ist es weiterhin möglich, die Anzahl der Punkte des dünnen Gitters und damit den bei Interpolationen, welche den Smolyak-Algorithmus verwenden, nötigen Datenaufwand $\mathrm{zu}$ formulieren. 


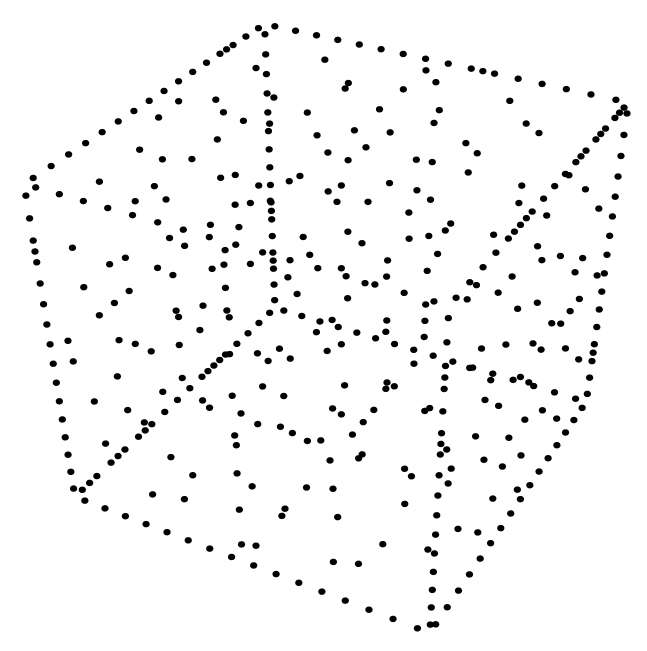

Abb.: Das dünne Gitter $H_{8,3}^{C C 2}$

Satz 3.17 Die Anzahl der Punkte $n(q, d)$ des dünnen Gitters $H_{q, d}$ mit $d, q \in I N$ und $q \geq d$ erfüllt

$$
n(q, d)=\sum_{\left(h_{1}, \ldots, h_{q-d+1}\right) \in J_{q-d+1, d}} \frac{d !}{h_{1} ! \cdot \ldots \cdot h_{q-d+1} !} \prod_{\zeta=1}^{q-d+1}\left(m_{\zeta}-m_{\zeta-1}\right)^{h_{\zeta}},
$$

wobei $m_{0}:=0$ gilt und die Indexmenge

$$
J_{k, d}:=\left\{h=\left(h_{1}, \ldots, h_{k}\right) \in\{0, \ldots, d\}^{k}: \sum_{l=1}^{k} h_{l}=d \text { und } \sum_{l=2}^{k}(l-1) h_{l} \leq k-1\right\}
$$

definiert ist.

Beweis: Zunächst soll das dünne Gitter in disjunkte Teilgitter zerlegt werden. Dazu bezeichnen wir $Y_{1}:=X_{1}$ und $Y_{l}:=X_{l} / X_{l-1}$ für $l \geq 1$. Da die Punktmengen $X_{l}$ sukzessive ineinander verschachtelt sind, sind diese Teilmengen $Y_{l}=\left\{x_{l m_{l-1}+1}, \ldots, x_{l m_{l}}\right\}$ disjunkt.

Weiterhin definieren wir die Teilgitter $G_{k_{1}, \ldots, k_{d}}:=Y_{k_{1}} \times \ldots \times Y_{k_{d}}$ für $k_{l} \in I N$; sie sind folglich ebenfalls disjunkt. Da diese Teilgitter mit Hilfe von Kreuzprodukten definiert sind, kann die Anzahl ihrer Gitterpunkte leicht bestimmt werden, sie lautet

$$
\left|G_{k_{1}, \ldots, k_{d}}\right|=\left(m_{k_{1}}-m_{k_{1}-1}\right)\left(m_{k_{2}}-m_{k_{2}-1}\right) \cdot \ldots \cdot\left(m_{k_{d}}-m_{k_{d}-1}\right) .
$$


Es gilt nun also

$$
\begin{aligned}
H_{q, d} & =\bigcup_{|k|_{d}=q}\left(X_{k_{1}} \times \ldots \times X_{k_{d}}\right) \\
& =\bigcup_{|k|_{d}=q}\left(\left(Y_{1} \cup \ldots \cup Y_{k_{1}}\right) \times \ldots \times\left(Y_{1} \cup \ldots \cup Y_{k_{d}}\right)\right) \\
& =\bigcup_{|k|_{d} \leq q}^{q}\left(Y_{k_{1}} \times \ldots \times Y_{k_{d}}\right) \\
& =\bigcup_{i=d}^{q} \bigcup_{|k|_{d}=i}\left(Y_{k_{1}} \times \ldots \times Y_{k_{d}}\right) \\
& =\bigcup_{i=d}^{q} \bigcup_{|k|_{d}=i} G_{k_{1}, \ldots, k_{d}} .
\end{aligned}
$$

Wegen (11) sollen nun diejenigen Teilgitter $G_{k_{1}, \ldots, k_{d}}$ und $G_{l_{1}, \ldots, l_{d}}$ zusammengefaßt werden, die die gleiche Anzahl von Gitterpunkten haben; also für die gilt

$$
k_{1}, \ldots, k_{d}=\sigma\left(l_{1}, \ldots, l_{d}\right)
$$

für jede Permutation $\sigma \in S_{d}$. Jede Ziffer $l$ tritt in solch einer Indexmenge mit einer bestimmten Häufigkeit $h_{l}$ auf; wir schreiben

$$
G_{k_{1}, \ldots, k_{d}}=G_{\sigma\left(h_{1} \sharp 1, h_{2} \sharp 2, \ldots, h_{q-d+1} \sharp(q-d+1)\right)} .
$$

Dabei gilt $h_{l} \in I_{0}$ mit $0 \leq h_{l} \leq d$ für alle $l$. Weiterhin handelt es sich bei den Gittern um ein kartesisches Produkt von d Punktmengen; daraus folgt also $\sum_{l=1}^{q-d+1} h_{l}=d$. Zusätzlich summieren sich in (12) die Indizes $k_{l}$ zu $i$, was auf $\sum_{l=1}^{q-d+1} l h_{l}=i$ schließen läßt. Aus kombinatorischen Überlegungen folgt schließlich, daß genau $\frac{d !}{h_{1} ! \cdot \ldots \cdot h_{q-d+1} !}$ Teilgitter die gemeinsame Anzahl von Punkten (11) besitzen. Zusammenfassend ergibt sich daraus

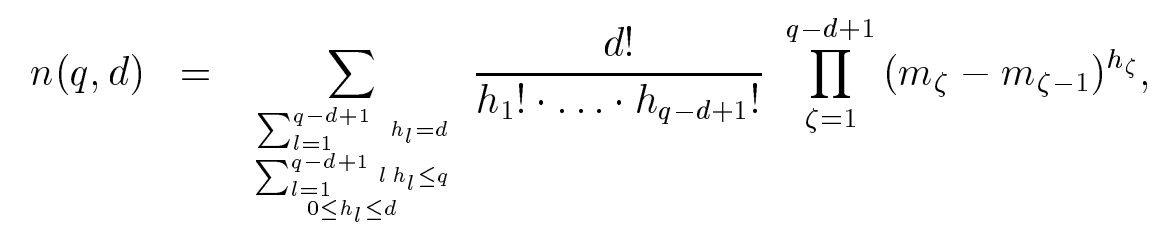

und damit folgt die Behauptung.

Bemerkung 3.18 Aus dem Beweis von Satz 3.17 wird gleichzeitig deutlich, daß wir das verschachtelte dünne Gitter auch als

$$
H_{q, d}=\bigcup_{|k|_{d} \leq q}\left(\left(X_{k_{1}} / X_{k_{1}-1}\right) \times \ldots \times\left(X_{k_{d}} / X_{k_{d}-1}\right)\right)
$$


schreiben können. Wir wenden in diesem Fall also den Smolyak-Operator - quasi als Smolyak-Kreuzproduktoperator - auf Datenmengen an, welche reellwertige (und damit eindimensionale) Datenpunkte enthalten, und erhalten auf diese Weise einen gitterartig strukturierten, $\mathbb{R}^{d}$-wertigen Datensatz.

Vielfach ist es sinnvoll, ein spezielles dünnes Gitter nach Clenshaw und Curtis zu benutzen. Hierbei gilt

$$
m_{1}=1 \text { und } m_{k}=2^{k-1}+1 \text { für } k \geq 2 ;
$$

wir nennen es das Clenshaw-Curtis-Gitter und bezeichnen es mit $H_{q, d}^{C C}$. In Anlehnung daran wird das dünne Gitter mit $m_{k}=2^{k-1}+1,1 \leq k \leq q-d+1$, mit $H_{q, d}^{C C 2}$ benannt.

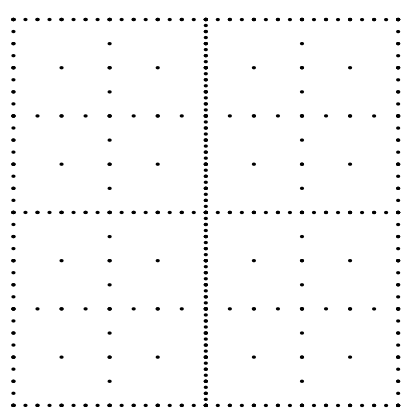

Abb.: Clenshaw-Curtis-Gitter $H_{8,2}^{C C}$

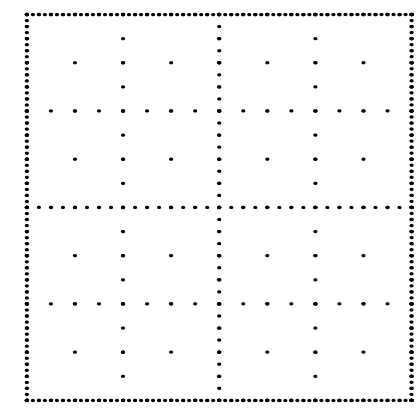

Abb.: Das dünne Gitter $H_{8,2}^{C C 2}$

Bemerkung 3.19 Wählt man das Clenshaw-Curtis-Gitter (13), so vereinfacht sich seine Gitterpunktanzahl zu

$$
n(q, d)=\sum_{i=0}^{q-d} 2^{i-d} \sum_{\left(h_{1}, \ldots, h_{q-d+1}\right) \in J_{q-d+1, d}^{i}} \frac{d !}{h_{1} ! \cdot \ldots \cdot h_{q-d+1} !} 2^{h_{1}+h_{2}}
$$

mit der Indexmenge

$$
J_{k, d}^{i}:=\left\{h=\left(h_{1}, \ldots, h_{k}\right) \in\{0, \ldots, d\}^{k}: \sum_{l=1}^{k} h_{l}=d \text { und } \sum_{l=1}^{k}(l-1) h_{l}=i\right\} .
$$


Beweis: Sei $k:=q-d$. Wir schreiben

$$
n(q, d)=\sum_{i=0}^{k} \sum_{\left(h_{1}, \ldots, h_{k+1}\right) \in J_{k+1, d}^{i}} \frac{d !}{h_{1} ! \cdot \ldots \cdot h_{k+1} !} \prod_{\zeta=1}^{k+1}\left(m_{\zeta}-m_{\zeta-1}\right)^{h_{\zeta}}
$$

und setzen ein

$$
\begin{aligned}
\prod_{\zeta=1}^{k+1}\left(m_{\zeta}-m_{\zeta-1}\right)^{h_{\zeta}} & =2^{h_{2}} \prod_{\zeta=3}^{k+1} 2^{(\zeta-2) h_{\zeta}}=2^{h_{2}+\sum_{\zeta=2}^{k+1}(\zeta-2) h_{\zeta}}=2^{h_{2}+\sum_{\zeta=2}^{k+1}(\zeta-1) h_{\zeta}-d+h_{1}} \\
& =2^{i-d+h_{1}+h_{2}} .
\end{aligned}
$$

Zur rechnergestützten Auswertung können folgende Formulierungen nützlich sein:

Korollar 3.20 Sei

$$
S_{k, d}:=\sum_{\left(h_{1}, \ldots, h_{k}\right) \in J_{k, d}^{k}} \frac{d !}{h_{1} ! \cdot \ldots \cdot h_{k} !} \prod_{\zeta=1}^{k}\left(m_{\zeta}-m_{\zeta-1}\right)^{h_{\zeta}}
$$

für alle $d, k \in \mathbb{N}$. Dann gilt für $q \in \mathbb{N}, q>d$

(1) $n(q, d)=n(q-1, d)+S_{q-d, d}+d m_{1}^{d-1}\left(m_{q-d+1}-m_{q-d}\right)$;

(2) $n(q, d)=m_{1}^{d}+\sum_{\zeta=1}^{q-d} S_{\zeta, d}+d m_{1}^{d-1}\left(m_{q-d+1}-m_{1}\right)$.

Beweis: Mit $q=k+d$ gilt

$$
\begin{aligned}
n(q, d) & =\sum_{\left(h_{1}, \ldots, h_{k+1}\right) \in J_{k+1, d}} \frac{d !}{h_{1} ! \cdot \ldots \cdot h_{k+1} !} \prod_{\zeta=1}^{k+1}\left(m_{\zeta}-m_{\zeta-1}\right)^{h_{\zeta}} \\
& =\sum_{\substack{\left(h_{1}, \ldots, h_{k+1} \in J_{k+1, d} \\
h_{k+1}=0\right.}} \frac{d !}{h_{1} ! \cdot \ldots \cdot h_{k+1} !} \prod_{\zeta=1}^{k+1}\left(m_{\zeta}-m_{\zeta-1}\right)^{h_{\zeta}} \\
& +\sum_{\substack{\left(h_{1}, \ldots, h_{k+1} \in J_{k+1, d} \\
h_{k+1} \geq 1\right.}} \frac{d !}{h_{1} ! \cdot \ldots \cdot h_{k+1} !} \prod_{\zeta=1}^{k+1}\left(m_{\zeta}-m_{\zeta-1}\right)^{h_{\zeta}} .
\end{aligned}
$$


Ist in der zweiten Summe $h_{k+1}>1$, so folgt wegen

$$
k \geq \sum_{l=1}^{k+1}(l-1) h_{l}>\sum_{l=1}^{k}(l-1) h_{l}+k \geq k
$$

ein Widerspruch. Damit kommt an dieser Stelle nur $h_{k+1}=1, h_{2}=\ldots=h_{k}=0$ und $h_{1}=d-1$ in Betracht. Der Ausdruck vereinfacht sich zu

$$
\begin{aligned}
n(k+d, d) & =n(k+d-1, d) \\
& +\sum_{\left(h_{1}, \ldots, h_{k}\right) \in J_{k, d}^{k}} \frac{d !}{h_{1} ! \cdot \ldots \cdot h_{k} !} \prod_{\zeta=1}^{k}\left(m_{\zeta}-m_{\zeta-1}\right)^{h_{\zeta}} \\
& +d m_{1}^{d-1}\left(m_{k+1}-m_{k}\right) .
\end{aligned}
$$

Induktiv erhalten wir damit auch den zweiten Teil der Behauptung.

An dieser Stelle wird die Wichtigkeit der Forderung $m_{1}=1$ deutlich, da für jede andere Wahl von $m_{1}$ die Gitterpunktanzahl mit steigender Dimension zu stark anwächst. Bereits in den Fällen $q=d$ und $q=d+1$ erhalten wir etwa die Ergebnisse $n(d, d)=m_{1}^{d}$ und $n(d+1, d)=m_{1}^{d}+d m_{1}^{d-1}\left(m_{2}-m_{1}\right)$.

Weiterhin formulieren wir wie bei Bemerkung 3.19:

Bemerkung 3.21 Im Clenshaw-Curtis-Fall $(13)$ gilt $n(d, d)=1, n(d+1, d)=1+2 d$ und für $q>d+1$

(1) $n(q, d)=n(q-1, d)+S_{q-d, d}+2^{q-d-1} d$;

(2) $n(q, d)=1+2^{q-d} d+\sum_{j=2}^{q-d} S_{j, d}$

mit

$$
S_{j, d}=2^{j-d} \sum_{\left(h_{1}, \ldots, h_{j}\right) \in J_{j, d}^{j}} \frac{d !}{h_{1} ! \cdot \ldots \cdot h_{j} !} 2^{h_{1}+h_{2}} .
$$

Daraus läßt sich das Verhalten der Gitterpunktanzahl für große Werte von $d$ folgern:

Lemma 3.22 Im Clenshaw-Curtis-Fall (13) gilt für feste $k$

$$
n(k+d, d)=\mathcal{O}\left(d^{k}\right), \quad d \rightarrow \infty .
$$


Beweis: Für große Werte von $d$ dominiert derjenige Summand von $S_{k, d}$, bei dem $h_{1}=d-k$ gilt. Dann folgt $\sum_{l=2}^{k} h_{l}=k=\sum_{l=2}^{k}(l-1) h_{l}$, durch Differenzbildung $\sum_{l=3}^{k}(l-2) h_{l}=0$ und damit $h_{2}=k$.

Das asymptotische Verhalten der Punktanzahl des dünnen Gitters für große Werte von $q$ finden wir bei Müller-Gronbach [23], dort wird das dünne Gitter $H_{q, d}^{C C 2}$ mit $m_{k}=2^{k-1}+1$ für alle $k \geq 1$, betrachtet.

Um die Gitterpunktanzahl von klassischem und dünnem Gitter vergleichen zu können, wählen wir das kleinste $d$-dimensionale klassische Gitter

$$
\tilde{H}_{q, d}=X_{q-d+1} \times \ldots \times X_{q-d+1},
$$

das unser dünnes Gitter $H_{q, d}$ noch enthält. Offensichtlich beträgt seine Knotenpunktanzahl $\left|\tilde{H}_{q, d}\right|:=m(q, d)=m_{q-d+1}^{d}$. Im Clenshaw-Curtis-Fall gilt also $m(q, d)=\left(2^{q-d}+1\right)^{d}$. Wir stellen nun beide Gitterpunktanzahlen in einigen konkreten Fällen gegenüber:

\begin{tabular}{|r|l|l|}
\hline$q-d$ & $n(q, d)$ & $m(q, d)$ \\
\hline \hline 0 & 1 & 1 \\
\hline 1 & $2 d+1$ & $3^{d}$ \\
\hline 2 & $2 d^{2}+2 d+1$ & $5^{d}$ \\
\hline 3 & $\frac{4}{3} d^{3}+2 d^{2}+\frac{14}{3} d+1$ & $9^{d}$ \\
\hline 4 & $\frac{2}{3} d^{4}+\frac{4}{3} d^{3}+\frac{22}{3} d^{2}+\frac{20}{3} d+1$ & $17^{d}$ \\
\hline 5 & $\frac{4}{15} d^{5}+\frac{2}{3} d^{4}+\frac{20}{3} d^{3}+\frac{34}{3} d^{2}+\frac{196}{15} d+1$ & $33^{d}$ \\
\hline 6 & $\frac{4}{45} d^{6}+\frac{4}{15} d^{5}+\frac{38}{9} d^{4}+\frac{32}{3} d^{3}+\frac{1246}{45} d^{2}+\frac{316}{15} d+1$ & $65^{d}$ \\
\hline 7 & $\frac{8}{315} d^{7}+\frac{4}{45} d^{6}+\frac{92}{45} d^{5}+\frac{62}{9} d^{4}+\frac{1376}{45} d^{3}+\frac{2206}{45} d^{2}+\frac{4132}{105} d+1$ & $129^{d}$ \\
\hline
\end{tabular}

Für spätere Anwendungen sind Abschätzungen der Punktanzahl eines dünnen Gitters wie etwa im folgenden Lemma von Wasilkowski und Woźniakowski [46] von Bedeutung:

Lemma 3.23 Sei $q \geq d$; die Größe des eindimensionalen Datensatzes $X_{i}$ erfülle für alle $j$ die Bedingung

$$
m_{j} \leq F_{0}\left(F^{j}-1\right)
$$

mit $F_{0}, F \in \mathbb{N}$. Dann gilt für verschränkte Datenmengen $X_{1} \subset X_{2} \subset \ldots \subset X_{q-d+1}$ die Ungleichung

$$
n(q, d) \leq F_{0}^{d}(F-1)^{d} \sum_{j=0}^{q-d} F^{j}\left(\begin{array}{c}
j+d-1 \\
d-1
\end{array}\right)
$$


mit Gleichheit im Fall $m_{j}=F_{0}\left(F^{j}-1\right)$. Daraus folgt

$$
n(q, d) \leq F_{0}^{d}\left(\frac{F-1}{F}\right)^{d} F^{q}\left(\begin{array}{l}
q-1 \\
d-1
\end{array}\right) \min \left(\frac{F}{F-1}, \frac{q}{d}\right) .
$$

Bei letzterer Abschätzung verwenden wir die Summenformel (A.1) aus dem Anhang.

Bemerkung 3.24 Da im Clenshaw-Curtis-Fall (13) Bedingung (15) mit $F_{0}=1$ und $F=2$ erfüllt ist, folgern wir also für seine Gitterpunktanzahl die obere Schranke

$$
\begin{aligned}
n(q, d) & \leq 2^{q-d}\left(\begin{array}{l}
q-1 \\
d-1
\end{array}\right) \min \left(2, \frac{q}{d}\right) \\
& \leq \frac{2^{-(d-1)}}{(d-1) !} 2^{q} q^{d-1} .
\end{aligned}
$$

Letztere Ungleichung ergibt sich aus $\left(\begin{array}{c}q-1 \\ d-1\end{array}\right) \leq \frac{1}{(d-1) !} q^{d-1}$, was man leicht induktiv beweisen kann. Eine andere Möglichkeit besteht darin, die ebenfalls induktiv beweisbare Abschätzung $n(d+k, d) \leq 2^{k+1} d^{k} z u$ wählen.

Verwenden wir ein dünnes Gitter mit $m_{j}=2^{j}, 1 \leq j \leq q-d+1$, so folgt

$$
n(q, d) \leq \frac{2^{q+1}}{(d-1) !} q^{d-1}
$$

Weiterhin folgern wir:

Korollar 3.25 Für alle $q \geq d, 0 \leq j \leq q-d$ gilt

$$
\sum_{\left(h_{1}, \ldots, h_{q-d+1}\right) \in J_{q-d+1, d}^{j}} \frac{d !}{h_{1} ! \cdot \ldots \cdot h_{q-d+1} !}=\left(\begin{array}{c}
j+d-1 \\
j
\end{array}\right) .
$$

Beweis: Wir verwenden (10) für den Spezialfall $m_{j}=F_{0}\left(F^{j}-1\right)$. Mit $\left(h_{1}, \ldots, h_{q-d+1}\right) \in J_{q-d+1, d}^{j}$ gilt $\left(m_{\zeta}-m_{\zeta-1}\right)^{h_{\zeta}}=F_{0}(F-1) F^{\zeta-1}$ und schließlich $\prod_{\zeta=1}^{q-d+1}\left(m_{\zeta}-m_{\zeta-1}\right)^{h_{\zeta}}=F_{0}^{d}(F-1)^{d} F^{j}$. Unter Verwendung von (16) folgt die Behauptung.

Damit kann die Gitterpunktanzahl eines weiteren speziellen dünnen Gitters exakt berechnet werden: 
Korollar 3.26 Die Punktanzahl eines verschachtelten dünnen Gitters $H_{q, d}$ mit $m_{j}=j \cdot m, m \in \mathbb{N}$, beträgt $m^{d}\left(\begin{array}{l}q \\ d\end{array}\right)$.

Auch erhalten wir mit Hilfe von Korollar 3.25 eine untere Schranke für das ClenshawCurtis-Gitter $H_{q, d}^{C C}$ mit (13):

Korollar 3.27 Im Clenshaw-Curtis-Fall (13) gilt $n(q, d) \geq \max \left(\left(\begin{array}{l}q \\ d\end{array}\right), 2^{q-2 d}\right)$.

Beweis: Wir verwenden Formulierung (14); mit $h_{1}, \ldots, h_{q-d+1} \in J_{q-d+1, d}^{i}$ schreiben wir

$$
2^{h_{1}+h_{2}}=2^{d-\sum_{j=3}^{q-d+1} h_{j}} \geq 2^{d-\sum_{j=3}^{q-d+1}(j-1) h_{j}}=2^{d-i+h_{2}} \geq 2^{d-i},
$$

damit gilt $n(q, d) \geq \sum_{i=0}^{q-d}\left(\begin{array}{c}d-1+i \\ i\end{array}\right)=\left(\begin{array}{l}q \\ d\end{array}\right)$. Weiterhin folgern wir mit $2^{h_{1}+h_{2}} \geq 1$ die untere Schranke

$$
n(q, d) \geq \sum_{i=0}^{q-d} 2^{i-d}\left(\begin{array}{c}
d-1+i \\
i
\end{array}\right)=2^{-d} \sum_{i=0}^{q-d} 2^{i}\left(\begin{array}{c}
d-1+i \\
i
\end{array}\right) \geq 2^{-d}\left(2^{q-d+1}-1\right) \geq 2^{q-2 d} .
$$

Ebenso ergibt sich:

Korollar 3.28 Sei $m_{j}-m_{j-1} \geq F_{0}(F-1) F^{j-1}$. Dann besitzt die Punktanzahl des zugehörigen dünnen Gitters $H_{q, d}$ die untere Schranke

$$
n(q, d) \geq F_{0}^{d}(F-1)^{d-1} \max \left(\min (1, F)^{q-d}(F-1)\left(\begin{array}{l}
q \\
d
\end{array}\right), F^{q-d+1}-1\right) .
$$

Beweis: Es gilt

$$
\begin{aligned}
n(q, d) & =\sum_{i=0}^{q-d} \sum_{\left(h_{1}, \ldots, h_{q-d+1}\right) \in J_{q-d+1, d}^{i}} \frac{d !}{h_{1} ! \cdot \ldots \cdot h_{d} !} \prod_{j=1}^{q-d+1}\left(m_{j}-m_{j-1}\right)^{h_{j}} \\
& \geq F_{0}^{d}(F-1)^{d} \sum_{i=0}^{q-d} \sum_{\left(h_{1}, \ldots, h_{q-d+1}\right) \in J_{q-d+1, d}^{i}} \frac{d !}{h_{1} ! \cdot \ldots \cdot h_{d} !} \prod_{j=1}^{q-d+1} F^{i} \\
& =F_{0}^{d}(F-1)^{d} \sum_{i=0}^{q-d}\left(\begin{array}{c}
i+d-1 \\
i
\end{array}\right) F^{i} .
\end{aligned}
$$


Einerseits folgern wir mit Formel (A.1) aus dem Anhang

$$
\begin{aligned}
n(q, d) & \geq F_{0}^{d}(F-1)^{d} \min (1, F)^{q-d} \sum_{i=0}^{q-d}\left(\begin{array}{c}
i+d-1 \\
i
\end{array}\right) \\
& =F_{0}^{d}(F-1)^{d} \min (1, F)^{q-d}\left(\begin{array}{l}
q \\
d
\end{array}\right) ;
\end{aligned}
$$

andererseits erreichen wir

$$
\begin{aligned}
n(q, d) & \geq F_{0}^{d}(F-1)^{d} \sum_{i=0}^{q-d} F^{i} \\
& =F_{0}^{d}(F-1)^{d-1}\left(F^{q-d+1}-1\right) .
\end{aligned}
$$

Fassen wir die vorangegangenen Ergebnisse zusammen, so erhalten wir untere und obere Schranken für $q$ in Abhängigkeit von der Gitterpunktanzahl $n(q, d)$ und der Raumdimension $d$ :

Korollar 3.29 Im Clenshaw-Curtis-Fall (13) gilt für die Gitterpunktanzahl $n=n(q, d)$

$$
\log ((d-1) !)+(d-1)-(d-1) \log (2 d+\log (n))+\log (n) \leq q \leq 2 d+\log (n) .
$$

Beweis: Die obere Schranke folgern wir unmittelbar aus Korollar 3.28. Mit Bemerkung 3.24 folgt

$$
\begin{aligned}
q & \geq \log \left(2^{d-1}(d-1) ! q^{-(d-1)} n\right) \\
& =d-1+\log (\ln ((d-1) !)+\log (n)-(d-1) \log (q) .
\end{aligned}
$$

Zusammen mit der oberen Schranke gilt

$$
q \geq d-1+\log (\ln ((d-1) !)+\log (n)-(d-1) \log (2 d+\log (n)) .
$$

Genauso folgern wir: 
Korollar 3.30 Im dünnen Gitter $H_{q, d}^{C C 2}$ mit $m_{j}=2^{j-1}+1,1 \leq j \leq q-d+1$ gilt für die Punktanzahl $n=n(q, d)$

$$
\log ((d-1) !)-1-(d-1) \log (2 d+\log (n))+\log (n) \leq q \leq 2 d+\log (n) .
$$

Beweis: Unter Verwendung von Korollar 3.28 folgern wir mit $F_{0}=1$ und $F=2$ die Schranke $n \geq 2^{q-d}$. Mit Bemerkung 3.24 gilt

$$
n \leq \frac{2^{q+1}}{(d-1) !}(2 d+\log (n))^{d-1}
$$

es folgt die Behauptung.

Es gilt auch:

Korollar 3.31 Gilt in einem dünnen Gitter $m_{j}=2^{j}, 1 \leq j \leq q-d+1$, so folgt für seine Punktanzahl $n:=n(q, d)$

$$
\log ((d-1) !)-1-(d-1) \log (d+\log (n))+\log (n) \leq q \leq d+\log (n) .
$$

Der Beweis ist analog zu denen von Korollar 3.29 und $3.30 \mathrm{zu}$ führen. Ebensolche Schranken gibt es in einem weiteren Fall:

Korollar 3.32 Sei $H_{q, d}$ ein dünnes Gitter mit $m_{j}=z \cdot j, 1 \leq j \leq q-d+1$. Dann gilt für seine Punktanzahl $n=n(q, d)$

$$
\frac{n^{\frac{1}{d}}}{z} \leq q \leq d \frac{n^{\frac{1}{d}}}{z}
$$

Beweis: Es gilt einerseits

$$
\left(\begin{array}{l}
q \\
d
\end{array}\right)=\prod_{j=0}^{d-1}\left(\frac{q-j}{d-j}\right)=\prod_{j=0}^{d-1}\left(1+\frac{q-d}{d-j}\right) \geq \prod_{j=0}^{d-1}\left(1+\frac{q-d}{d}\right)=\left(\frac{q}{d}\right)^{d},
$$

andererseits folgern wir auf analoge Weise

$$
\left(\begin{array}{l}
q \\
d
\end{array}\right) \leq q^{d}
$$




\subsection{Smolyakfunktionen}

Unter Verwendung des Algorithmus von Smolyak kann weiterhin eine neue Art von Funktionen konstruiert werden:

Definition 3.33 Es seien $\phi_{1}, \ldots, \phi_{q-d+1}$ reellwertige, univariate Funktionen. Dann heißt die d-variate Funktion

$$
A_{q, d}\left(\phi_{1}, \ldots, \phi_{q-d+1}\right)=\sum_{|j|_{d} \leq q}\left(\phi_{j_{1}}-\phi_{j_{1}-1}\right) \otimes \ldots \otimes\left(\phi_{j_{d}}-\phi_{j_{d}-1}\right)
$$

$q$-te Smolyakfunktion der Dimension d mit zugrundeliegenden Funktionen $\phi_{1}, \ldots, \phi_{q-d+1}$ und $\phi_{0}=0$.

Analog zu Satz 3.15 gilt offensichtlich

$$
A_{q, d}\left(\phi_{1}, \ldots, \phi_{q-d+1}\right)=\sum_{q-d+1 \leq|j|_{d} \leq q}(-1)^{q-|j|_{d}}\left(\begin{array}{c}
d-1 \\
q-|j|_{d}
\end{array}\right)\left(\phi_{j_{1}} \otimes \ldots \otimes \phi_{j_{d}}\right) .
$$

Stimmen die univariaten Funktionen überein, so wird die Smolyakfunktion zur Tensorproduktfunktion:

Lemma 3.34 Die univariate, reellwertige Funktion $\phi$ erfüllt für alle $x=\left(x_{1}, \ldots, x_{d}\right)$ $\in \mathbb{R}^{d}$ die Gleichung

$$
A_{q, d}(\phi, \ldots, \phi)(x)=\prod_{j=1}^{d} \phi\left(x_{j}\right)=A_{d, d}(\phi) .
$$

Eine weitere Smolyakfunktion wird im folgenden berechnet:

Lemma 3.35 Seien $\phi$ und $\psi$ univariate und reellwertige Funktionen. Dann gilt

$$
\begin{aligned}
A_{q, d}(\phi, \ldots, \phi, \psi)= & \psi \otimes \phi \otimes \ldots \otimes \phi+\ldots+\phi \otimes \ldots \otimes \phi \otimes \psi \\
& -(d-1) \phi \otimes \ldots \otimes \phi .
\end{aligned}
$$

Beweis: Aus praktischen Gründen schreiben wir jede Tensorproduktfunktion $\bigotimes_{j_{1}=1}^{n_{1}} \phi_{1} \otimes \ldots \otimes \bigotimes_{j_{m}=1}^{n_{m}} \phi_{m}$ als $\otimes\left(\phi_{1} \sharp n_{1}, \ldots, \phi_{m} \sharp n_{m}\right)$, wobei $\phi_{1}, \ldots, \phi_{m}$ univariate Funktionen bezeichnen. Wir zeigen nun induktiv, daß die Gleichung

$$
\begin{aligned}
& A_{q, d}(\phi, \ldots, \phi, \psi) \\
& \quad=A_{q-u, d-u}(\phi, \ldots, \phi, \psi) \otimes \bigotimes(\phi \sharp u)+\sum_{l=0}^{u-1} \bigotimes(\phi \sharp d-l-1,(\psi-\phi) \sharp 1, \phi \sharp l)
\end{aligned}
$$


für $1 \leq u \leq d-1$ gilt. Mit $\phi_{0}=0, \phi_{1}=\ldots=\phi_{q-d}=\phi$ und $\phi_{q-d+1}=\psi$ folgern wir für die Differenzfunktionen $\Delta_{1}=\phi, \Delta_{2}=\ldots=\Delta_{q-d} \equiv 0$ und $\Delta_{q-d+1}=\psi-\phi$. Aufgrund von Lemma 3.16 schreiben wir nun

$$
\begin{aligned}
A_{q, d}(\phi, \ldots, \phi, \psi) & =\sum_{l=d-1}^{q-2} A_{l, d-1}(\phi, \ldots, \phi) \otimes \Delta_{q-l}+A_{q-1, d-1}(\phi, \ldots, \phi, \psi) \otimes \Delta_{1} \\
& =A_{d-1, d-1}(\phi) \otimes(\psi-\phi)+A_{q-1, d-1}(\phi, \ldots, \phi, \psi) \otimes \phi \\
& =\bigotimes(\phi \amalg d-1) \otimes(\psi-\phi)+A_{q-1, d-1}(\phi, \ldots, \phi, \psi) \otimes \phi .
\end{aligned}
$$

Genauso folgt induktiv

$$
\begin{aligned}
A_{q, d}(\phi, \ldots, \phi, \psi)-\sum_{l=0}^{u-2} \bigotimes(\phi \sharp d-l-1, \psi-\phi \sharp 1, \phi \sharp l) \\
=A_{q-u+1, d-u+1}(\phi, \ldots, \phi, \psi) \otimes \bigotimes(\phi \sharp u-1) \\
=A_{q-u, d-u}(\phi, \ldots, \phi, \psi)(\phi, \ldots, \phi, \psi) \otimes \bigotimes(\phi \sharp u)+ \\
\quad+\bigotimes(\phi \sharp d-u) \otimes(\psi-\phi) \otimes \bigotimes(\phi \sharp u-1) .
\end{aligned}
$$

Wir schlußfolgern daraus mit $u=d-1$

$$
\begin{aligned}
& A_{q, d}(\phi, \ldots, \phi, \psi) \\
& =A_{q-d+1,1}(\phi, \ldots, \phi, \psi) \otimes \bigotimes(\phi \sharp d-1)+\sum_{l=0}^{d-2} \bigotimes(\phi \sharp d-l-1,(\psi-\phi) \sharp 1, \phi \sharp l) \\
& =\bigotimes(\psi \sharp 1, \phi \sharp d-1)+\sum_{l=0}^{d-2} \bigotimes(\phi \sharp d-l-1, \psi \sharp 1, \phi \sharp l)-(d-1) \bigotimes(\phi \sharp d) .
\end{aligned}
$$

Bemerkung 3.36 Damit gilt $A_{q, d}(\phi, \ldots, \phi, \psi)=A_{d+1, d}(\phi, \psi)$.

Lemma 3.35 läßt sich unter Beachtung der vorangehenden Bemerkung 3.36 noch weiter verallgemeinern. Im zugehörigen Beweis zeigen wir die Richtigkeit von Lemma 3.35 noch auf eine andere Weise.

Lemma 3.37 Für die reellwertigen Funktionen $\phi, \psi_{1}, \ldots, \psi_{l}$ mit $1 \leq l \leq q-d+1$ gilt

$$
A_{q, d}\left(\phi, \ldots, \phi, \psi_{1}, \ldots, \psi_{l}\right)=A_{d+l, d}\left(\phi, \psi_{1}, \ldots, \psi_{l}\right)
$$


Beweis: Mit Hilfe von Lemma 3.16 schreiben wir

$$
\begin{aligned}
A_{q, d}( & \left.\phi, \ldots, \phi, \psi_{1}, \ldots, \psi_{l}\right)-A_{d+l, d}\left(\phi, \psi_{1}, \ldots, \psi_{l}\right) \\
= & \sum_{j=d-1}^{q-1} A_{j, d-1} \otimes \Delta_{q-j}\left(\phi, \ldots, \phi, \psi_{1}, \ldots, \psi_{l}\right)-\sum_{j=d-1}^{d+l-1} A_{j, d-1} \otimes \Delta_{d+l-j}\left(\phi, \psi_{1}, \ldots, \psi_{l}\right) \\
= & A_{q-1, d-1}\left(\phi, \ldots, \phi, \psi_{1}, \ldots, \psi_{l}\right) \otimes \phi+A_{d+l-2, d-1}\left(\phi, \ldots, \phi, \psi_{1}, \ldots, \psi_{l}\right) \otimes\left(\psi_{1}-\phi\right) \\
& +\sum_{j=2}^{l} A_{d+l-j-1, d-1}(\phi, \ldots) \otimes\left(\psi_{j}-\psi_{j-1}\right) \\
& -A_{d+l-1, d-1}\left(\phi, \psi_{1}, \ldots, \psi_{l}\right) \otimes \phi-A_{d+l-2, d-1}(\phi, \ldots) \otimes\left(\psi_{1}-\phi\right) \\
& -\sum_{j=2}^{l} A_{d+l-j-1, d-1} \otimes\left(\psi_{j}-\psi_{j-1}\right) \\
= & \left(A_{q-1, d-1}\left(\phi, \ldots, \phi, \psi_{1}, \ldots, \psi_{l}\right)-A_{d+l-1, d-1}\left(\phi, \psi_{1}, \ldots, \psi_{l}\right)\right) \otimes \phi .
\end{aligned}
$$

Daraus folgern wir induktiv

$$
\begin{aligned}
& A_{q, d}\left(\phi, \ldots, \phi, \psi_{1}, \ldots, \psi_{l}\right)-A_{d+l, d}\left(\phi, \psi_{1}, \ldots, \psi_{l}\right) \\
& \quad=\left(A_{q-u, d-u}\left(\phi, \ldots, \phi, \psi_{1}, \ldots, \psi_{l}\right)-A_{d+l-u, d-u}\left(\phi, \psi_{1}, \ldots, \psi_{l}\right)\right) \otimes \bigotimes(\phi \sharp u) ;
\end{aligned}
$$

mit $u=d-1$ folgt schließlich

$$
\begin{aligned}
& A_{q, d}\left(\phi, \ldots, \phi, \psi_{1}, \ldots, \psi_{l}\right)-A_{d+l, d}\left(\phi, \psi_{1}, \ldots, \psi_{l}\right) \\
& \quad=\left(A_{q-d+1,1}\left(\phi, \ldots, \phi, \psi_{1}, \ldots, \psi_{l}\right)-A_{l+1,1}\left(\phi, \psi_{1}, \ldots, \psi_{l}\right)\right) \otimes \bigotimes(\phi \sharp d-1) \\
& =\left(\psi_{l}-\psi_{l}\right) \otimes \bigotimes(\phi \sharp d-1) \\
& =0 .
\end{aligned}
$$

Einige spezielle Smolyakfunktionen entstehen, wenn die zugrundegelegten Funktionen univariate Polynome sind. Wir definieren:

Definition 3.38 Für $q, d, m_{1}, \ldots, m_{q-d+1} \in \mathbb{N}$ und $q \geq d$ heißt

$$
\mathcal{S} \mathcal{P}_{q, d}^{m_{1}, \ldots, m_{q-d+1}}:=\bigcup_{|i|_{d} \leq q}\left(\mathbb{P}_{m_{i_{1}}} \otimes \cdots \otimes \mathbb{P}_{m_{i_{d}}}\right)
$$

Smolyak-Polynomraum. 
Offenbar gilt $\mathcal{S P}_{q, d}^{m_{1}, \ldots, m_{q-d+1}}=\mathcal{S V}_{q, d}^{\mathbb{P}_{m_{1}}, \ldots, \mathbb{P}_{m_{q-d+1}}}$. Wir folgern:

Lemma 3.39 Sei $q \geq d \geq 1, m_{j} \in \mathbb{N}$ für $1 \leq j \leq q-d+1$. Dann gilt

$$
A_{q, d}\left(p_{1}, \ldots, p_{q-d+1}\right) \in \mathcal{S P}_{q, d}^{m_{1}, \ldots, m_{q-d+1}}
$$

falls $p_{j} \in \mathbb{P}_{m_{j}}$. 


\section{Interpolation auf dem dünnen Gitter}

Mit Hilfe des Smolyak-Tensorproduktoperators ist es möglich, Interpolanten für Daten auf einem passenden dünnen Gitter zu finden. Das folgende Lemma verdeutlicht die Zusammenhänge.

Lemma 4.1 Seien $\mathcal{F}_{j} \subseteq \mathcal{F}$ und $\mathcal{V}_{j} \subseteq \mathcal{V}$ für $1 \leq j \leq q-d+1$ Vektorräume; weiterhin sei $f \in \mathcal{S V}_{q, d}^{\mathcal{F}_{1}, \ldots, \mathcal{F}_{q-d+1}}$ eine $d$-variate Funktion. Für $1 \leq j \leq q-d+1$ bezeichnen $U_{j}$ lineare Operatoren $U_{j}: \mathcal{F}_{j} \rightarrow \mathcal{V}_{j} ;$ mit $I_{l}=I_{1} \otimes \ldots \otimes I_{1}$ sei ein l-variater Tensorproduktoperator, der den Tensorraum $\mathcal{F} \otimes \ldots \otimes \mathcal{F}$ auf den Tensorraum $\mathcal{V} \otimes \ldots \otimes \mathcal{V}$ abbildet, benannt. Sei $H_{q, d}$ ein verschachteltes dünnes Gitter, welches auf den univariaten Datensätzen $X_{1} \subseteq X_{2} \subseteq \ldots \subseteq X_{q-d+1}$ basiert; es gelte

$$
U_{j} f_{j}(x)=I_{1} f_{j}(x)
$$

für alle $x \in X_{j}, f_{j} \in \mathcal{F}_{j}$. Dann folgt

$$
A_{q, d} f(x)=I_{d} f(x)
$$

für alle $x \in H_{q, d}$.

Beweis: Gilt $x=\left(\tilde{x}, x_{d}\right) \in H_{q, d}$, so folgern wir $\tilde{x} \in H_{q-k, d-1}$ und $x_{d} \in X_{k}$ für $1 \leq k \leq q-d+1$. Wir können dabei $k$ so wählen, daß $\tilde{x} \in H_{q-k, d-1} \backslash H_{q-k-1, d-1}$ gilt. Mit Lemma 3.16 folgt induktiv

$$
\begin{aligned}
A_{q, d} f(x) & \sum_{l=d-1}^{q-1}\left(A_{l, d-1} \otimes \Delta_{q-l}\right) f(x) \\
= & \sum_{l=d-1}^{q-k-1}\left(A_{l, d-1} \otimes \Delta_{q-l}\right) f(x)+\sum_{l=q-k}^{q-1}\left(A_{l, d-1} \otimes \Delta_{q-l}\right) f(x) \\
= & \sum_{l=d-1}^{q-k-1}\left(A_{l, d-1} \otimes U_{q-l}\right) f\left(\tilde{x}, x_{d}\right)-\sum_{l=d-1}^{q-k-1}\left(A_{l, d-1} \otimes U_{q-l-1}\right) f\left(\tilde{x}, x_{d}\right)+ \\
& +\sum_{l=q-k}^{q-1}\left(I_{d-1} \otimes \Delta_{q-l}\right) f\left(\tilde{x}, x_{d}\right) \sum_{l=d-1}^{q-k-1}\left(A_{l, d-1} \otimes I_{1}\right) f\left(\tilde{x}, x_{d}\right)-\sum_{l=d-1}^{q-k-1}\left(A_{l, d-1} \otimes I_{1}\right) f\left(\tilde{x}, x_{d}\right)+
\end{aligned}
$$




$$
\begin{aligned}
& +\sum_{l=q-k}^{q-1}\left(I_{d-1} \otimes \Delta_{q-l}\right) f\left(\tilde{x}, x_{d}\right) \\
= & \left(I_{d-1} \otimes U_{k}\right) f\left(\tilde{x}, x_{d}\right) \\
= & I_{d} f(x) .
\end{aligned}
$$

Ein ähnlicher Beweis findet sich unter nicht ganz so allgemeinen Bedingungen bei [5]. Ein Beispiel für einen Operator $I_{l}$ stellt die Einbettungsabbildung des Raumes $\mathcal{F} \otimes \ldots \otimes \mathcal{F}$ in den Raum $\mathcal{V} \otimes \ldots \otimes \mathcal{V}$ dar, denn unter Ausnutzung der Eindeutigkeit dieser Abbildung folgern wir, daß sie ein Tensorproduktoperator, bestehend aus den univariaten Einbettungsabbildungen, sein muß. Es folgt dann $A_{q, d} f=f$ und wir erhalten damit die Möglichkeit, aus univariaten Interpolanten auf den Datensätzen $X_{1}, \ldots, X_{q-d+1}$ multivariate Interpolanten auf dem dünnen Gitter zu konstruieren. Andere Operatoren $I_{l}$ dieser Art werden beispielsweise bei der numerischen Integration benutzt.

Um Tensorproduktoperatoren definieren zu können, benötigen wir die Tensorräume $\mathcal{F}_{j_{1}} \otimes \ldots \otimes \mathcal{F}_{j_{d}}$ bzw. $\mathcal{V}_{j_{1}} \otimes \ldots \otimes \mathcal{V}_{j_{d}}$. Der nächste Satz nach Wasilkowski und Woźniakowski [46] zeigt, daß sich für eine Fehlerabschätzung bei der Interpolation mit Hilfe des Smolyak-Algorithmus noch eine weitere Eigenschaft als nützlich erweist.

Satz 4.2 Es seien $\mathcal{F}_{j} \subseteq \mathcal{F}$ und $\mathcal{V}_{j} \subseteq \mathcal{V}$ für $1 \leq j \leq q-d+1$ Banachräume; die Norm des zugehörigen l-variaten Tensorräume $\left(\mathcal{F} \otimes \ldots \otimes \mathcal{F},\|\cdot\|_{\mathcal{F} \otimes \ldots \otimes \mathcal{F}}\right)$ sei mit

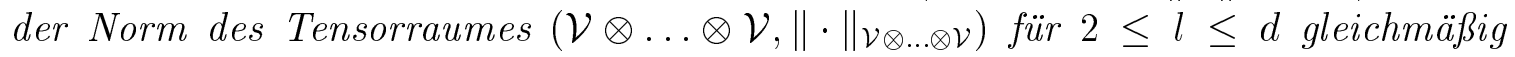
verträglich. Dann gilt für den d-variaten Tensorproduktoperator $I_{d}=I_{1} \otimes \ldots \otimes I_{1}$ die Fehlerabschätzung

$$
\begin{aligned}
\| I_{d}- & A_{q, d} \|_{\mathcal{F} \otimes \ldots \otimes \mathcal{F}, \mathcal{V} \otimes \ldots \otimes \mathcal{V}} \\
\leq & \left\|I_{1}-U_{q-d+1}\right\|_{\mathcal{F}, \mathcal{V}}\left\|I_{1}\right\|_{\mathcal{F}, \mathcal{V}}^{d-1}+ \\
& +\sum_{k=1}^{d-1} \sum_{|j|_{k} \leq q-d+k} \prod_{\mu=1}^{k}\left\|I_{1}\right\|_{\mathcal{F}, \mathcal{V}}^{d-1-k}\left(\left\|I_{1}-U_{j_{\mu}}\right\|_{\mathcal{F}, \mathcal{V}}+\left\|I_{1}-U_{j_{\mu}-1}\right\|_{\mathcal{F}, \mathcal{V}}\right) \\
& \cdot\left\|I_{1}-U_{q-d+k+1-|j|_{k}}\right\|_{\mathcal{F}, \mathcal{V} .}
\end{aligned}
$$

Beweis: Wir bezeichnen das $j$-fache Tensorprodukt $\mathcal{F} \otimes \ldots \otimes \mathcal{F}$ mit $\mathcal{F}_{\mathbf{j}}$ bzw. $\mathcal{V} \otimes \ldots \otimes \mathcal{V}$ mit $\mathcal{V}_{\mathbf{j}}$. Wegen Lemma 3.16 gilt

$$
\left\|I_{d}-A_{q, d}\right\|_{\mathcal{F}_{\mathbf{d}}, \mathcal{V}_{\mathbf{d}}}
$$




$$
\begin{aligned}
= & \left\|I_{d-1} \otimes I_{1}-\sum_{l=d-1}^{q-1} \Delta_{j_{1}} \otimes \ldots \otimes \Delta_{j_{d-1}} \otimes U_{q-l}\right\|_{\mathcal{F}_{\mathbf{d}}, \mathcal{V}_{\mathbf{d}}} \\
= & \left\|\left(I_{d-1}-A_{q-1, d-1}\right) \otimes I_{1}+\sum_{|j|_{d-1} \leq q-1} \Delta_{j_{1}} \otimes \ldots \otimes \Delta_{j_{d-1}} \otimes\left(I_{1}-U_{q-|j|_{d-1}}\right)\right\|_{\mathcal{F}_{\mathbf{d}}, \mathcal{V}_{\mathbf{d}}} \\
\leq & \left\|\left(I_{d-1}-A_{q-1, d-1}\right) \otimes I_{1}\right\|_{\mathcal{F}_{\mathbf{d}}, \mathcal{V}_{\mathbf{d}}}+\sum_{|j|_{d-1} \leq q-1}\left\|\Delta_{j_{1}} \otimes \ldots \otimes \Delta_{j_{d-1}} \otimes\left(I_{1}-U_{q-|j|_{d-1}}\right)\right\|_{\mathcal{F}_{\mathbf{d}}, \mathcal{V}_{\mathbf{d}}} \\
= & \left\|I_{d-1}-A_{q-1, d-1}\right\|_{\mathcal{F}_{\mathbf{d}-\mathbf{1}}, \mathcal{V}_{\mathbf{d}-\mathbf{1}}}\left\|I_{1}\right\|_{\mathcal{F}, \mathcal{V}}+ \\
& +\sum_{|j|_{d-1} \leq q-1}\left\|\Delta_{j_{1}}\right\|_{\mathcal{F}, \mathcal{V}} \cdot \ldots \cdot\left\|\Delta_{j_{d-1}}\right\|_{\mathcal{F}, \mathcal{V}} \cdot\left\|I_{1}-U_{q-|j|_{d-1}}\right\|_{\mathcal{F}, \mathcal{V}} \\
\leq & \left\|I_{d-1}-A_{q-1, d-1}\right\|_{\mathcal{F}_{\mathbf{d}-\mathbf{1}}, \mathcal{V}_{\mathbf{d}-\mathbf{1}}}\left\|I_{1}\right\|_{\mathcal{F}, \mathcal{V}}+ \\
& +\sum_{|j|_{d-1} \leq q-1} \prod_{\mu=1}^{d-1}\left(\left\|I_{1}-U_{j_{\mu}}\right\|_{\mathcal{F}, \mathcal{V}}+\left\|I_{1}-U_{j_{\mu}-1}\right\|_{\mathcal{F}, \mathcal{V}}\right) \cdot\left\|I_{1}-U_{q-|j|_{d-1}}\right\|_{\mathcal{F}, \mathcal{V}}
\end{aligned}
$$

aufgrund von $\left\|\Delta_{j}\right\|_{\mathcal{F}, \mathcal{V}}=\left\|I_{1}-U_{j-1}-I_{1}+U_{j}\right\|_{\mathcal{F}, \mathcal{V}} \leq\left\|I_{1}-U_{j}\right\|_{\mathcal{F}, \mathcal{V}}+\left\|I_{1}+U_{j-1}\right\|_{\mathcal{F}, \mathcal{V}}$ Induktiv folgern wir mit $1 \leq \tilde{k} \leq d-1$

$$
\begin{aligned}
\| A_{q, d} & \|_{\mathcal{F}_{\mathbf{d}}, \mathcal{V}_{\mathbf{d}}} \\
\leq & \left\|I_{d-\tilde{k}}-A_{q-\tilde{k}, d-\tilde{k}}\right\|_{\mathcal{F}_{\mathbf{d}-\tilde{\mathbf{k}}}, \mathcal{V}_{\mathbf{d}-\tilde{\mathbf{k}}}}\left\|I_{1}\right\|_{\mathcal{F}, \mathcal{V}}^{\tilde{k}}+ \\
& +\sum_{k=1}^{\tilde{k}}\left\|I_{1}\right\|_{\mathcal{F}, \mathcal{V}}^{k-1} \sum_{|j|_{d-k} \leq q-k} \prod_{\mu=1}^{d-k}\left(\left\|I_{1}-U_{j_{\mu}}\right\|_{\mathcal{F}, \mathcal{V}}+\left\|I_{1}-U_{j_{\mu}-1}\right\|_{\mathcal{F}, \mathcal{V}}\right) \cdot \\
& \cdot\left\|I_{1}-U_{q-k+1-|j|_{d-k}}\right\|_{\mathcal{F}, \mathcal{V}} \\
= & \left\|I_{1}-U_{q-d+1}\right\|_{\mathcal{F}, \mathcal{V}}\left\|I_{1}\right\|_{\mathcal{F}, \mathcal{V}}^{d-1}+ \\
& +\sum_{k=1}^{d-1}\left\|I_{1}\right\|_{\mathcal{F}, \mathcal{V}}^{k-1} \sum_{|j|_{d-k} \leq q-k} \prod_{\mu=1}^{d-k}\left(\left\|I_{1}-U_{j_{\mu}}\right\|_{\mathcal{F}, \mathcal{V}}+\left\|I_{1}-U_{j_{\mu}-1}\right\|_{\mathcal{F}, \mathcal{V}}\right) \cdot \\
& \cdot\left\|I_{1}-U_{q-k+1-|j|_{d-k}}\right\|_{\mathcal{F}, \mathcal{V}} \\
= & \left\|I_{1}-U_{q-d+1}\right\|_{\mathcal{F}, \mathcal{V}}\left\|I_{1}\right\|_{\mathcal{F}, \mathcal{V}}^{d-1}+ \\
& +\sum_{k=1}^{d-1}\left\|I_{1}\right\|_{\mathcal{F}, \mathcal{V}}^{d-k-1} \sum_{|j|_{k} \leq q-d+k} \prod_{\mu=1}^{k}\left(\left\|I_{1}-U_{j_{\mu}}\right\|_{\mathcal{F}, \mathcal{V}}+\left\|I_{1}-U_{j_{\mu}-1}\right\|_{\mathcal{F}, \mathcal{V}}\right) . \\
& \cdot\left\|I_{1}-U_{q-d+k+1-|j|_{k}}\right\|_{\mathcal{F}, \mathcal{V}}
\end{aligned}
$$

falls wir $\tilde{k}=d-1$ wählen. Es folgt die Behauptung.

Gilt $\mathcal{F} \subseteq \mathcal{V}$ und ist $I_{d}$ auf dem Unterraum $\mathcal{F} \otimes \ldots \otimes \mathcal{F}$ die Identitätsabbildung dieses Raumes auf sich selbst, so können wir diese Fehlerabschätzung für die Interpolation 
auf dem dünnen Gitter nutzen. Im Fall $\left\|I_{1}\right\|_{\mathcal{F}, \mathcal{V}}=1$ gilt

$$
\begin{aligned}
\| I_{d}- & A_{q, d} \|_{\mathcal{F} \otimes \ldots \otimes \mathcal{F}, \mathcal{V} \otimes \ldots \otimes \mathcal{V}} \\
\leq & \left\|I_{1}-U_{q-d+1}\right\|_{\mathcal{F}, \mathcal{V}}+ \\
& \quad+\sum_{k=1}^{d-1} \sum_{|j|_{k} \leq q-d+k} \prod_{\mu=1}^{k}\left(\left\|I_{1}-U_{j_{\mu}}\right\|_{\mathcal{F}, \mathcal{V}}+\left\|I_{1}-U_{j_{\mu-1}}\right\|_{\mathcal{F}, \mathcal{V}}\right) \cdot\left\|I_{1}-U_{q-d+k+1-|j|_{k}}\right\|_{\mathcal{F}, \mathcal{V}}
\end{aligned}
$$

Haben die univariaten Fehlerschranken eine spezifische Gestalt, so kann man die Interpolante auf folgenden Art und Weise darstellen:

Korollar 4.3 Es gelten neben den Voraussetzungen von Satz 4.2 die Fehlerabschätzungen

$$
\left\|I_{1}-U_{j}\right\|_{\mathcal{F}, \mathcal{V}} \leq c \cdot B^{j} \quad \text { und } \quad\left\|I_{1}\right\|_{\mathcal{F}, \mathcal{V}} \leq K
$$

Dann folgt

$$
\left\|I_{d}-A_{q, d}\right\|_{\mathcal{F} \otimes \ldots \otimes \mathcal{F}, \mathcal{V} \otimes \ldots \otimes \mathcal{V}} \leq c B^{q-d+1}\left(\begin{array}{c}
q \\
d-1
\end{array}\right) \max (K, c(1+B))^{d-1} .
$$

Beweis: Es ist mit Formel (A.3) aus dem Anhang

$$
\begin{aligned}
& \left\|I_{d}-A_{q, d}\right\|_{\mathcal{F} \otimes \ldots \otimes \mathcal{F}, \mathcal{V} \otimes \ldots \otimes \mathcal{V}} \\
& \leq c B^{q-d+1} K^{d-1}+\sum_{k=1}^{d-1} K^{d-1-k} \sum_{|j|_{k} \leq q-d+k} \prod_{\mu=1}^{k}\left(c\left(B^{j_{\mu}}+B_{j^{\mu}-1}\right)\right) c B^{q-d+k+1-|j|_{k}} \\
& =c B^{q-d+1} K^{d-1}+\sum_{k=1}^{d-1} c^{k+1} K^{d-1-k} \sum_{|j|_{k} \leq q-d+k} \prod_{\mu=1}^{k}\left(B^{j_{\mu}}+B^{j_{\mu}-1}\right) B^{q-d+k+1-|j|_{k}} \\
& =c B^{q-d+1} K^{d-1}+\sum_{k=1}^{d-1} c^{k+1} K^{d-1-k} \sum_{|j|_{k} \leq q-d+k} \prod_{\mu=1}^{k}\left(1+B^{-1}\right) B^{j_{\mu}} B^{q-d+k+1-|j|_{k}} \\
& =c B^{q-d+1}\left(1+\sum_{k=1}^{d-1}\left(\frac{c}{K}\right)^{k}(1+B)^{k}\right) \sum_{|j|_{k} \leq q-d+k} 1 \\
& =c B^{q-d+1} K^{d-1} \sum_{k=0}^{d-1}\left(\frac{c}{K}\right)^{k}(1+B)^{k}\left(\begin{array}{c}
k+q-d \\
k
\end{array}\right) \\
& \leq c B^{q-d+1} K^{d-1} \max \left(1,\left(\frac{c}{K}\right)(1+B)\right)^{d-1} \sum_{k=0}^{d-1}\left(\begin{array}{c}
k+q-d \\
k
\end{array}\right) \\
& =c B^{q-d+1}\left(\begin{array}{c}
q \\
d-1
\end{array}\right) \max (K, c(1+B))^{d-1} .
\end{aligned}
$$


In Abhängigkeit von der Gitterpunktanzahl des Clenshaw-Curtis-Gitters folgt daraus:

Korollar 4.4 Unter den Voraussetzungen von Korollar 4.3 gilt mit $0<B \leq 1$

$$
\begin{aligned}
& \left\|I_{d}-A_{q, d}\right\|_{\mathcal{F} \otimes \ldots \otimes \mathcal{F}, \mathcal{V} \otimes \ldots \otimes \mathcal{V}} \\
& \quad \leq n^{\log (B)}(2 d+\log (B))^{(d-1)(-\log (B)+1)} \frac{c}{(d-1) !} B^{\log ((d-1) !)} \max (K, c(1+B))^{d-1} .
\end{aligned}
$$

Dabei bezeichnet $n:=n(q, d)$ die Anzahl der Punkte des dünnen Gitters nach Clenshaw und Curtis. Im Fall $B=2^{-l}$ folgern wir

$$
\begin{aligned}
& \left\|I_{d}-A_{q, d}\right\|_{\mathcal{F} \otimes \ldots \otimes \mathcal{F}, \mathcal{V} \otimes \ldots \otimes \mathcal{V}} \\
& \quad \leq n^{-l}(2 d+\log (n))^{(d-1)(l+1)} \frac{c}{(d-1) ! !^{l+1}} \max \left(K, c\left(1+2^{-l}\right)\right)^{d-1} .
\end{aligned}
$$

Beweis: Mit Korollar 3.29 gilt

$$
\begin{aligned}
B^{(q-d+1) l} & \leq B^{\log ((d-1) !) l} \cdot B^{l \log \left(n(2 d+\log (n))^{-(d-1)}\right)} \\
& =B^{\log ((d-1) !) l} \cdot n^{l \log (B)}(2 d+\log (n))^{-(d-1) l \log (B)}
\end{aligned}
$$

und wir folgern wegen

$$
\left(\begin{array}{c}
q \\
d-1
\end{array}\right) \leq \frac{q^{d-1}}{(d-1) !} \leq \frac{(2 d+\log (n))^{d-1}}{(d-1) !}
$$

die Behauptung.

\subsection{Untersuchung geeigneter Funktionenräume und ihrer Nor- men}

Um Satz 4.2 bei der Fehlerabschätzung von Smolyak-Interpolanten anwenden zu können, müssen die Normen der zugrundegelegten Tensorräume gleichmäßig verträglich sein. Im folgenden Satz finden wir einen Tensorraum mit dieser Eigenschaft, der aufgrund seiner besonderen Struktur auf vielfältige Art und Weise angewandt werden kann. 
Satz 4.5 Es gilt

$$
\mathbb{R} \otimes \ldots \otimes \mathbb{R} \simeq \mathbb{R}
$$

und jede Kreuznorm $\|\cdot\|_{\alpha, \mathbb{R} \otimes \ldots \otimes \mathbb{R}}$ erfüllt für $z=\sum_{j=1}^{n} x_{j} \otimes y_{j}$ mit $x_{j} \in \mathbb{R}$, $y_{j} \in \mathbb{R} \otimes \ldots \otimes \mathbb{R}$ die Gleichung

$$
\|z\|_{\alpha, \mathbb{R} \otimes \ldots \otimes \mathbb{R}}=\left|\sum_{j=1}^{n} x_{j} y_{j}\right| .
$$

Beweis: Wir führen den Beweis für ein einfaches Tensorprodukt. Es sei $z \in \mathbb{R} \otimes \mathbb{R}$; mit Hilfe der Basis $\{1\}$ von $\mathbb{R}$ schreiben wir

$$
\begin{aligned}
z & =\sum_{j=1}^{n} x_{j} \otimes y_{j}=\sum_{j=1}^{n} x_{j} \otimes\left(1 \cdot y_{j}\right)=\sum_{j=1}^{n}\left(x_{j} \cdot y_{j}\right) \otimes 1 \\
& =\sum_{j=1}^{n}\left(x_{j} \cdot y_{j} \cdot 1\right) \otimes 1=\sum_{j=1}^{n}\left(x_{j} \cdot y_{j}\right)(1 \otimes 1) .
\end{aligned}
$$

Offenbar stellt damit $\{1 \otimes 1\}$ eine Basis von $\mathbb{R} \otimes \mathbb{R}$ dar, und wir können eine Isomorphie $F: \mathbb{R} \otimes \mathbb{R} \rightarrow \mathbb{R}$ mit

$$
F\left(\sum_{j=1}^{n} x_{j} \otimes y_{j}\right)=\sum_{j=1}^{n} x_{j} y_{j}
$$

definieren.

Nach [36] gilt mit $z \in X_{1} \otimes \ldots \otimes X_{d}$ für jede Kreuznorm $\|\cdot\|_{\alpha, X_{1}, \ldots, X_{d}}$ auf $X_{1} \otimes \ldots \otimes X_{d}$ die Ungleichung

$$
\|z\|_{\lambda, X_{1} \otimes \ldots \otimes X_{d}} \leq\|z\|_{\alpha, X_{1} \otimes \ldots \otimes X_{d}} \leq\|z\|_{\gamma, X_{1} \otimes \ldots \otimes X_{d}} .
$$

Wir zeigen nun, daß $\|z\|_{\lambda, \mathbb{R} \otimes \mathbb{R}} \geq\|z\|_{\gamma, \mathbb{R} \otimes \mathbb{R}}$ erfüllt ist und folgern damit die Behauptung. Es gilt mit $z=\sum_{j=1}^{n} x_{j} \otimes y_{j}$

$$
\begin{aligned}
\|z\|_{\lambda, \mathbb{R} \otimes \mathbb{R}} & =\sup _{\substack{\phi \in \mathbb{R}^{*} \\
\|\phi\|_{\mathbb{R}^{*}=1}}}\left|\sum_{j=1}^{n} \phi\left(x_{j}\right) y_{j}\right|=\sup _{\substack{\phi \in \mathbb{R}^{*} \\
\|\phi\|_{\mathbb{R}^{*}=1}}}|\phi(1)|\left|\sum_{j=1}^{n} x_{j} y_{j}\right| \\
& =\sup _{\substack{\phi \in \mathbb{R}^{*} \\
\text { a }(x)|=1 \\
x \in \mathbb{R},| x \mid=1}}|\phi(1)|\left|\sum_{j=1}^{n} x_{j} y_{j}\right|=\left|\sum_{j=1}^{n} x_{j} y_{j}\right|
\end{aligned}
$$




$$
\begin{aligned}
& \geq \inf _{\substack{z=\sum_{\begin{subarray}{c}{j=1 \\
x_{j} \in \mathbb{R}, y_{j} \in \mathbb{R}} }}^{n} x_{j} y_{j} \otimes 1}\end{subarray}}\left|\sum_{j=1}^{n} x_{j} y_{j}\right||1|=\left\|\sum_{j=1}^{n} x_{j} y_{j} \otimes 1\right\|_{\gamma, \mathbb{R} \otimes \mathbb{R}} \\
& =\left\|\sum_{j=1}^{n} x_{j} \otimes y_{j}\right\|_{\gamma, \mathbb{R} \otimes \mathbb{R}}=\|z\|_{\gamma, \mathbb{R} \otimes \mathbb{R}}
\end{aligned}
$$

Bemerkung 4.6 Damit gilt für jede Kreuznorm $\|\cdot\|_{\alpha, \mathbb{R} \otimes \ldots \otimes \mathbb{R}}$ die Isomorphie

$$
\mathbb{R} \otimes_{\alpha} \ldots \otimes_{\alpha} \mathbb{R} \simeq \mathbb{R}
$$

und wir können diesen Tensorraum in Verbindung mit jedem anderen, auf Basis einer gleichmäßig verträglichen Kreuznorm bestehenden Tensorraum nutzen, was für die Anwendung auf Punktauswertungen von Bedeutung ist.

Der kommende Satz stellt eine Erweiterung eines Theorems aus [18], welches Aussagen über stetige $d$-variate Funktionen beinhaltet, dar. Wir benötigen dazu zunächst

Definition 4.7 Sei $d \in \mathbb{N}, r=\left(r_{1}, \ldots, r_{d}\right) \in \mathbb{N}_{0}^{d}$ und $U \subseteq \mathbb{R}^{d}$ offen. Dann benennen wir den Raum aller Funktionen $f: U \rightarrow \mathbb{R}$, die im l-ten Argument, $1 \leq l \leq d$, $r_{l}$-fach stetig differenzierbar sind, mit $\mathcal{C}_{d}^{r}(U)$. Gilt $r_{1}=\ldots=r_{d}=: \tilde{r}$, so schreiben $\operatorname{wir} \mathcal{C}_{d}^{\tilde{r}}(U)$.

Satz 4.8 Sei der Raum

$$
\mathcal{F}_{d}^{r}(S):=\left\{f \in \mathcal{C}_{d}^{r}(S):\|f\|_{\mathcal{F}_{d}^{r}(s)}<\infty\right\}
$$

mit $S \subseteq \mathbb{R}^{d}$

$$
\|f\|_{\mathcal{F}_{d}^{r}}:=\sup _{\substack{\alpha=\left(\alpha_{1}, \ldots, \alpha_{d}\right) \in N_{0}^{d} \\ \alpha_{l} \in\left\{0, \ldots, r_{l}\right\}}}\left\|D^{\alpha} f\right\|_{\infty, S}<\infty
$$

und $r=\left\{r_{1}, \ldots, r_{d}\right\}$ bzw. $r \in N_{0}$ definiert. Es seien $S_{j} \subset \mathbb{R}^{d_{j}}, 1 \leq j \leq \tilde{d}$, kompakt. Dann gilt mit $R_{j} \in I_{0}^{d_{j}}$ und $r=\left(r_{1}, \ldots, r_{\tilde{d}}\right)$

$$
\mathcal{F}_{d_{1}}^{r_{1}}\left(S_{1}\right) \otimes_{\lambda} \ldots \otimes_{\lambda} \mathcal{F}_{d_{\tilde{d}}}^{r_{\tilde{d}}}\left(S_{\tilde{d}}\right) \simeq \mathcal{F}_{d_{1}+\ldots+d_{\tilde{d}}}^{r}\left(S_{1} \times \ldots \times S_{\tilde{d}}\right) .
$$


Beweis: Aufgrund von Satz 3.2 führen wir den Beweis für $\tilde{d}=2$. Wir zeigen zunächst, daß die Abbildung

$$
\begin{aligned}
F: \mathcal{F}_{d_{1}}^{r_{1}}\left(S_{1}\right) \otimes_{\lambda} \mathcal{F}_{d_{2}}^{r_{2}}\left(S_{2}\right) & \rightarrow \mathcal{F}_{d}^{r}\left(S_{1} \times S_{2}\right) \\
f & \mapsto F_{f}
\end{aligned}
$$

mit $f=\sum_{j=1}^{n} f_{1 j} \otimes f_{2 j}$ und

$$
F_{f}\left(s_{1}, s_{2}\right)=\sum_{j=1}^{n} f_{1 j}\left(s_{1}\right) f_{2 j}\left(s_{2}\right)
$$

für $s_{1} \in S_{1}$ und $s_{2} \in S_{2}$ isometrisch ist. Es gilt

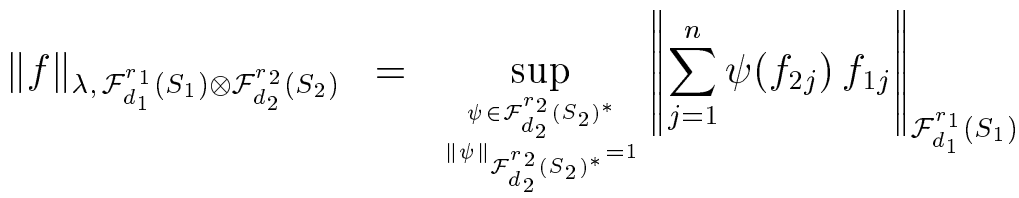

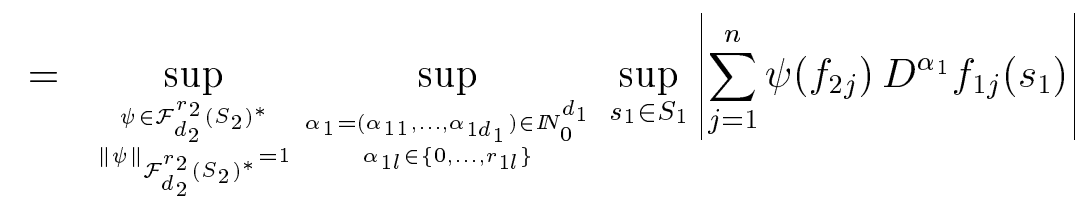

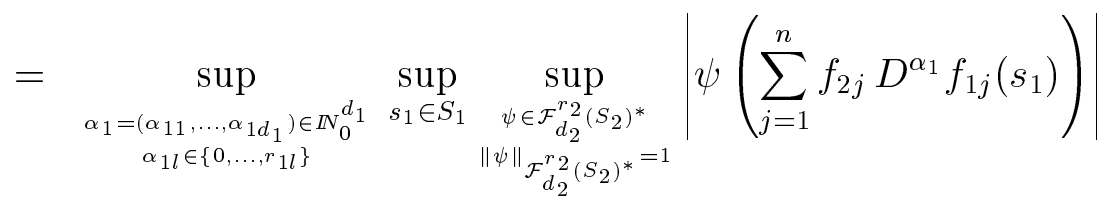

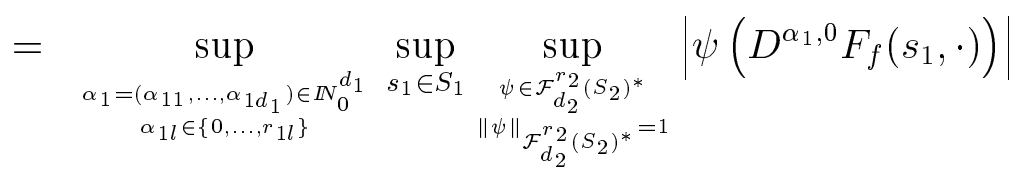

$$
\begin{aligned}
& =\sup _{\substack{\alpha_{1}=\left(\alpha_{11}, \ldots, \alpha_{1 d_{1}}\right) \in \mathbb{N}_{0}^{d_{1}} \\
\alpha_{1 l} \in\left\{0, \ldots, r_{1 l}\right\}}} \sup _{s_{1} \in S_{1}}\left\|D^{\alpha_{1}, 0} F_{f}\left(s_{1}, \cdot\right)\right\|_{\mathcal{F}_{d_{2}}^{r_{2}}\left(S_{2}\right)} \\
& =\sup _{\substack{\alpha_{j}=\left(\alpha_{j 1}, \ldots, \alpha_{j d_{j}}\right) \in \mathbb{N}_{0} d_{j} \\
\alpha_{j l} \in\left\{0, \ldots, r_{j l}\right\}, j=1,2}} \sup _{\substack{s_{1} \in S_{1} \\
s_{2} \in S_{2}}}\left|D^{\alpha_{1}, \alpha_{2}} F_{f}\left(s_{1}, s_{2}\right)\right| \\
& =\left\|F_{f}\right\|_{\mathcal{F}_{d}^{r}\left(S_{1} \times S_{2}\right)} \text {. }
\end{aligned}
$$

Wir zeigen nun die Dichtheit von $F\left(\mathcal{F}_{d_{1}}^{r_{1}}\left(S_{1}\right) \otimes \mathcal{F}_{d_{2}}^{r_{2}}\left(S_{2}\right)\right)$ in $\mathcal{F}_{d}^{r}\left(S_{1} \times S_{2}\right)$ und folgern damit die Behauptung. Wir wählen $g \in \mathcal{F}_{d}^{r}\left(S_{1} \times S_{2}\right)$. Die Funktion $\tilde{g}\left(s_{1}\right):=g\left(s_{1}, \cdot\right)$ beschreibt eine stetige Abbildung $\tilde{g}: S_{1} \rightarrow \mathcal{F}_{d_{2}}^{r_{2}}\left(S_{2}\right)$. Da $S_{1}$ kompakt ist, folgt auch die Kompaktheit von $\tilde{g}\left(S_{1}\right)$. Da $\tilde{g}\left(S_{1}\right)$ als metrischer Raum interpretiert werden kann, folgern wir damit, daß $\tilde{g}\left(S_{1}\right)$ präkompakt ist, d.h., $\tilde{g}\left(S_{1}\right)$ läßt sich durch endlich viele 
Kugeln mit beliebigem Radius $\epsilon>0$ überdecken. Wir bezeichnen diese Kugeln mit den Mittelpunktfunktionen $f_{2 j} \in \mathcal{F}_{d_{2}}^{r_{2}}\left(S_{2}\right)$ als

$$
B\left(f_{2 j}, \epsilon\right)=\left\{h \in \mathcal{F}_{d_{2}}^{r_{2}}\left(S_{2}\right):\left\|h-f_{2 j}\right\|_{\mathcal{F}_{d_{2}}^{r_{2}\left(S_{2}\right)}}<\epsilon\right\}
$$

und benennen ihre Anzahl mit $n$. Auf einem Kompaktum lassen sich nach [10] beliebig oft stetig differenzierbare Funktionen $g_{1}, \ldots, g_{n}: \tilde{g}\left(S_{1}\right) \rightarrow[0,1]$ finden, die eine Zerlegung der Eins $\sum_{j=1}^{n} g_{j}=1$ darstellen und für $1 \leq j \leq n$ die Bedingung $g_{j}(x)=0$ für alle $x \in \tilde{g}\left(S_{1}\right) \backslash B\left(f_{2 j}, \epsilon\right)$ erfüllen. Wir können nun die Funktionen

$$
f_{1 j}:=g_{j} \circ \tilde{g}: S_{1} \rightarrow[0,1]
$$

konstruieren; sie sind im $l$-ten Argument für alle $l\left(r^{T} e_{l}\right)$-fach stetig differenzierbar, wobei $e_{l}$ den $l$-ten Einheitsvektor darstellt, und erfüllen ebenfalls die Bedingung

$$
\sum_{j=1}^{n} f_{1 j}=1
$$

Weiterhin gilt $f_{1 j}\left(s_{1}\right)=0$, falls $\left\|\tilde{g}\left(s_{1}\right)-f_{2 j}\right\|_{\mathcal{F}_{d_{2}}^{r_{2}}\left(S_{2}\right)} \geq \epsilon$. Daraus folgern wir

$$
\begin{aligned}
& \left\|g-\sum_{j=1}^{n} f_{1 j} \otimes f_{2 j}\right\|_{\mathcal{F}_{d}^{r}\left(S_{1} \times S_{2}\right)}=\max _{\substack{\alpha_{1}=\left(\alpha_{11}, \ldots, \alpha_{\left.1 d_{1}\right) \in N_{0} d_{1}} \alpha_{1 j} \in\left\{0, \ldots, r_{1 j}\right\} \\
\alpha_{1} \in S_{1}\right.}} \sup _{s_{1}}\left\|g\left(s_{1}, \cdot\right)-\sum_{j=1}^{n} f_{1 j}\left(s_{1}\right) \otimes f_{2 j}\right\|_{\mathcal{F}_{d_{2}}^{r_{2}}\left(S_{2}\right)}
\end{aligned}
$$

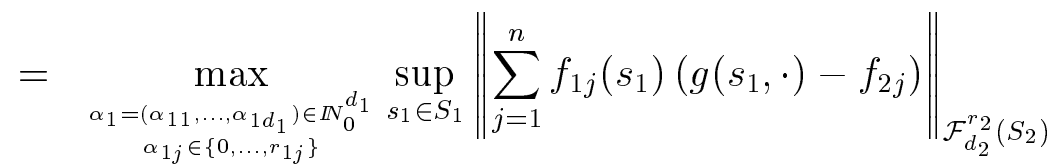

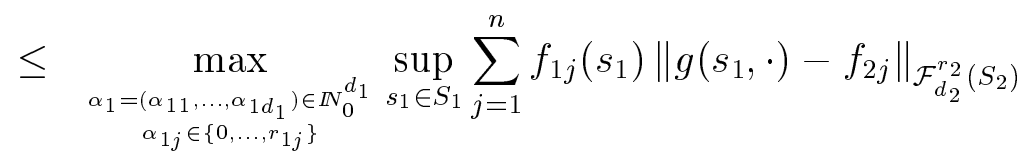

$$
\begin{aligned}
& <\max _{\substack{\alpha_{1}=\left(\alpha_{11}, \ldots, \alpha_{1 d_{1}}\right) \in N_{N}^{d_{1}} \\
\alpha_{1 j} \in\left\{0, \ldots, r_{1 j}\right\}}} \sup _{s_{1} \in S_{1}} \sum_{j=1}^{n} f_{1 j}\left(s_{1}\right) \epsilon \\
& =\epsilon
\end{aligned}
$$

und das Bild von $\mathcal{F}_{d_{1}}^{r_{1}}\left(S_{1}\right) \otimes \mathcal{F}_{d_{2}}^{r_{2}}\left(S_{2}\right)$ ist dicht in $\mathcal{F}_{d_{2}}^{r_{2}}\left(S_{2}\right)$.

Nun können wir also Satz 4.2 mit $S_{j} \subseteq S, \tilde{S}_{j} \subseteq \tilde{S}, \mathcal{F}_{j}=\mathcal{F}_{d_{j}}^{r_{j}}\left(S_{j}\right)$ und $\mathcal{V}_{j}=\mathcal{F}_{d_{j}}^{s_{j}}\left(\tilde{S}_{j}\right)$ benutzen. Bei der numerischen Integration mit dem Smolyak-Algorithmus, vgl. [25], wendet man diese Räume für $n=d, r=r_{1}=\ldots=r_{d}$ und $S_{j}=\tilde{S}_{j}=[-1,1]$, $1 \leq j \leq d$, unter Nutzung von Satz 4.5 an. 
Bemerkung 4.9 Genauso können wir für den Funktionenraum $\mathcal{F}_{d}^{r}(S)$ die Norm

$$
\|f\|_{\mathcal{F}_{d}^{r, k}}:=\left(\sum_{\substack{\alpha=\left(\alpha_{1}, \ldots, \alpha_{d}\right) \in N_{0}^{d} \\ \alpha_{l} \in\left\{0, \ldots, r_{l}\right\}}}\left\|D^{\alpha} f\right\|_{\infty, S}^{k}\right)^{\frac{1}{k}}
$$

mit $f \in \mathcal{F}_{d}^{r}(S)$ wählen.

Auf analoge Weise kann man auch das folgende Ergebnis zeigen.

Satz 4.10 Sei $U$ eine offene Menge; seien $S_{j}, 1 \leq j \leq \tilde{d}$, offene Teilmengen des $\mathbb{R}^{d_{j}}$. Bezeichne $\mathcal{C}_{d, c}^{r}(U)$ den Raum der Funktionen aus $\mathcal{C}_{d}^{r}(U)$ mit kompaktem Träger. Dann ist der Tensorraum $\mathcal{C}_{d_{1}, c}^{r_{1}}\left(S_{1}\right) \otimes_{\lambda} \ldots \otimes_{\lambda} \mathcal{C}_{d_{\tilde{d}}, c}^{r_{\tilde{d}}}\left(S_{\tilde{d}}\right)$ mit $d=d_{1}+\ldots+d_{\tilde{d}}$ und $r=\left\{r_{1}, \ldots, r_{\tilde{d}}\right\}$ dicht in $\mathcal{C}_{d}^{r}\left(S_{1} \times \ldots \times S_{\tilde{d}}\right)$. Ist $\mathcal{D}(U)$ der Raum der unendlich oft differenzierbaren Funktionen auf $U$ mit kompakten, in $U$ enthaltenen Trägern, so ist $\mathcal{D}\left(S_{1}\right) \otimes_{\lambda} \ldots \otimes_{\lambda} \mathcal{D}\left(S_{\tilde{d}}\right)$ dicht in $\mathcal{D}\left(S_{1} \times \ldots \times S_{\tilde{d}}\right)$.

Der Beweis ist auch in [45] zu finden. Daraus folgt das für spätere Anwendungen bedeutende

Korollar 4.11 Sei

$$
\mathcal{S}_{d}=\left\{\gamma \in C^{\infty}\left(\mathbb{R}^{d}\right) \quad: \quad \lim _{\|x\| \rightarrow \infty}\left|x^{k} D^{l} \gamma(x)\right|=0 \quad \text { für alle } k, l \in N_{0}^{d}\right\}
$$

der Schwartzsche Raum. Dann ist der Tensorraum $\mathcal{S}_{d_{1}} \otimes_{\lambda} \ldots \otimes_{\lambda} \mathcal{S}_{d_{\tilde{d}}}$ dicht in $\mathcal{S}_{d_{1}+\ldots+d_{\tilde{d}}}$.

Beweis: Wir definieren eine Funktion

$$
\gamma=\sum_{j=1}^{n} \gamma_{1 j} \otimes \gamma_{2 j} \quad \in \mathcal{S}_{d_{1}} \otimes \mathcal{S}_{d_{2}}
$$

mit $\gamma_{1 j} \in \mathcal{S}_{d_{1}}$ und $\gamma_{2 j} \in \mathcal{S}_{d_{2}}$. Offenbar gilt

$$
\lim _{\|x\| \rightarrow \infty}\left|x^{k} D^{l} \gamma_{z}(x)\right|=\lim _{\substack{\left\|x_{1}\right\| \rightarrow \infty \\\left\|x_{2}\right\| \rightarrow \infty}}\left|\sum_{n=1}^{j} x_{1}^{k_{1}} D^{l_{1}} \gamma_{1 j}\left(x_{1}\right) \cdot x_{2}^{k_{2}} D^{l_{2}} \gamma_{2 j}\left(x_{2}\right)\right|=0
$$

und es ist $\mathcal{S}_{d_{1}} \otimes \mathcal{S}_{d_{2}} \subseteq \mathcal{S}_{d}$. Nach [2] ist $\mathcal{D}(S)$ für alle $S \subset \mathbb{R}^{d}$ im Schwartzschen Raum $\mathcal{S}_{d}$ enthalten und ist dort dicht, weiterhin gilt Satz 4.10. Damit ist einerseits also auch der Tensorraum $\mathcal{D}\left(S_{1}\right) \otimes_{\lambda} \mathcal{D}\left(S_{2}\right)$ mit $S_{1} \subset \mathbb{R}^{d_{1}}$ und $S_{2} \subset \mathbb{R}^{d_{2}}$ dicht in $\mathcal{S}_{d}$, 
andererseits folgt die Dichtheit von $\mathcal{D}\left(S_{1}\right) \otimes \mathcal{D}\left(S_{2}\right)$ in $\mathcal{S}_{d_{1}} \otimes_{\lambda} \mathcal{S}_{d_{2}}$. Insgesamt folgern wir die Behauptung.

Weitere Beispiele finden wir, wenn wir Tensorprodukt-Hilberträume mit der HilbertraumKreuznorm aus Lemma 3.14 verwenden.

Satz 4.12 Seien $S_{j} \subseteq \mathbb{R}^{d_{j}}$ Mengen, die aus abzählbaren meßbaren Mengen bestehen. Dann folgt

$$
L_{2}\left(S_{1}\right) \otimes_{\beta} \ldots \otimes_{\beta} L_{2}\left(S_{d}\right) \simeq L_{2}\left(S_{1} \times \ldots \times S_{d}\right)
$$

Der Satz wird in [18] bewiesen. Ähnliche Resultate gelten auch für Räume vom Sobolev-Typ. Dazu benötigen wir zunächst

Definition 4.13 Sei $S \subseteq \mathbb{R}^{d}$ und $r \in \mathbb{N}_{0}^{d}$. Dann definieren wir

$$
\mathcal{F}_{d, L_{2}}^{r}(S)=\left\{f \in L_{2}(S):\|f\|_{\mathcal{F}_{d, L_{2}}^{r}(S)}<\infty\right\}
$$

mit

$$
\|f\|_{\mathcal{F}_{d, L_{2}}^{r}(S)}=\sup _{\substack{\alpha=\left(\alpha_{1}, \ldots, \alpha_{d}\right) \in \mathbb{N}_{0}^{d} \\ \alpha_{j} \in\left\{0, \ldots, r_{j}\right\}}}\left\|D^{\alpha} f\right\|_{L_{2}(S)}
$$

für alle $f \in \mathcal{F}_{d, L_{2}}^{r}(S)$.

Satz 4.14 Es gilt für aus abzählbaren meßbaren Mengen bestehende $S_{j} \subseteq \mathbb{R}^{d_{j}}$, $1 \leq j \leq \tilde{d}$, und $r=\left(r_{1}, \ldots, r_{\tilde{d}}\right)$

$$
\mathcal{F}_{d_{1}, L_{2}}^{r_{1}}\left(S_{1}\right) \otimes_{\beta} \ldots \otimes_{\beta} \mathcal{F}_{d_{\tilde{d}}, L_{2}}^{r_{\tilde{d}}}\left(S_{\tilde{d}}\right) \simeq \mathcal{F}_{d_{1}+\ldots+d_{\tilde{d}}, L_{2}}^{r}\left(S_{1} \times \ldots \times S_{\tilde{d}}\right) .
$$

Beweis: Wir zeigen zunächst, daß $\mathcal{F}_{d, L_{2}}^{r}$ ein Hilbertraum mit Skalarprodukt

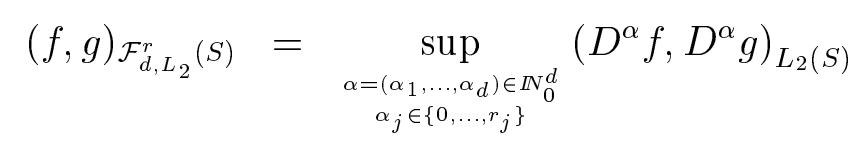

ist. Da sich Symmetrie, Bilinearität und positive Definitheit vom $L_{2}$-Skalarprodukt übertragen, muß nur noch die Vollständigkeit gezeigt werden. Es sei also $\left\{f_{k}\right\}_{k \in \mathbb{N}}$ eine Cauchyfolge in $\mathcal{F}_{d, L_{2}}^{r}(S)$. Dann ist $\left\{D^{\alpha} f_{k}\right\}_{k \in \mathbb{N}}$ für alle $\alpha \in \mathbb{N}_{0}^{d}$ eine Cauchyfolge in $L_{2}(S)$. Da $L_{2}(S)$ vollständig ist, existiert $f^{\alpha} \in L_{2}(S)$ mit

$$
D^{\alpha} f_{k} \rightarrow\|\cdot\|_{L_{2}(S)} \quad f^{\alpha}
$$


insbesondere gilt $f_{k} \rightarrow\|\cdot\|_{L_{2}(S)} f^{0}=: \quad f$; und wegen Abgeschlossenheit der schwachen Ableitungsabbildung folgt $D^{\alpha} f=f^{\alpha}$. Damit gilt $f_{k} \rightarrow_{\mathcal{F}_{d, L_{2}}^{r}(S)} f$.

Ohne Einschränkung sei $\tilde{d}=2$. Wir definieren nun eine Abbildung $F$ auf $\mathcal{F}_{d_{1}, L_{2}}^{r_{1}}\left(S_{1}\right) \otimes \mathcal{F}_{d_{2}, L_{2}}^{r_{2}}\left(S_{2}\right)$ mit $F(z)=f_{z}$, wobei

$$
f_{z}\left(s_{1}, s_{2}\right)=\sum_{j=1}^{n} x_{j}\left(s_{1}\right) y_{j}\left(s_{2}\right)
$$

für alle $s_{1} \in S_{1}, s_{2} \in S_{2}$ und $z=\sum_{j=1}^{n} x_{j} \otimes y_{j} \in \mathcal{F}_{d_{1}, L_{2}}^{r_{1}}\left(S_{1}\right) \otimes \mathcal{F}_{d_{2}, L_{2}}^{r_{2}}\left(S_{2}\right)$ gelte. Da der Satz von Fubini angewandt werden kann, folgern wir

$$
\begin{aligned}
& \left\|f_{z}\right\|_{\mathcal{F}_{d_{1}+d_{2}, L_{2}}^{r}}=\sup _{\substack{\alpha=\left(\alpha_{1}, \ldots, \alpha_{d}\right) \in \mathbb{N}_{0}^{d} \\
\alpha_{j} \in\left\{0, \ldots, r_{e_{j}}\right\}}} \int_{S_{1} \times S_{2}} D^{\alpha} f^{2}\left(s_{1}, s_{2}\right) d\left(s_{1}, s_{2}\right) \\
& =\sup _{\substack{\alpha=\left(\alpha_{1}, \ldots, \alpha_{d}\right) \in \mathbb{N}_{0}^{d} \\
\alpha_{j} \in\left\{0, \ldots, r^{T} e_{j}\right\}}} \int_{S_{1}} \int_{S_{2}}\left(D^{\alpha} f\left(s_{1}, s_{2}\right)\right)^{2} d s_{2} d s_{1} \\
& =\sup _{\substack{\alpha=\left(\alpha_{1}, \ldots, \alpha_{d}\right) \in N_{0}^{d} \\
\alpha_{j} \in\left\{0, \ldots, r T^{T}\right\}}} \int_{\left.S_{1}\right\}} \int_{S_{2}} \sum_{\substack{1 \leq j \leq n \\
1 \leq k \leq n}} D^{\alpha}\left(x_{j}\left(s_{1}\right) y_{j}\left(s_{2}\right)\right) D^{\alpha}\left(x_{k}\left(s_{1}\right) y_{k}\left(s_{2}\right)\right) d s_{2} d s_{1} \\
& =\sum_{\substack{1 \leq j \leq n \\
1 \leq k \leq n}} \sup _{\substack{\tilde{\alpha}_{1}=\left(\alpha_{1}, \ldots, \alpha_{d_{1}}\right) \in N_{0}^{d_{1}} \\
\alpha_{j} \in\left\{0, \ldots, r T_{\left.e_{j}\right\}}\right\}}} \int_{S_{1}} D^{\tilde{\alpha}_{1}} x_{j}\left(s_{1}\right) D^{\tilde{\alpha}_{1}} x_{k}\left(s_{1}\right) d s_{1} .
\end{aligned}
$$

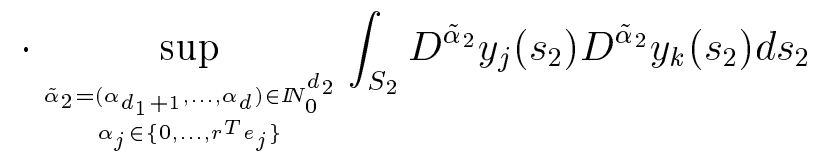

$$
\begin{aligned}
& =\sum_{\substack{1 \leq j \leq n \\
1 \leq k \leq n}}\left(x_{j}, x_{k}\right)_{\mathcal{F}_{d_{1}, L_{2}}^{r_{1}}\left(S_{1}\right)}\left(y_{j}, y_{k}\right)_{\mathcal{F}_{d_{2}, L_{2}}^{r_{2}}}\left(S_{2}\right) \\
& =\|z\|_{\beta, \mathcal{F}_{d_{1}, L_{2}}^{r_{1}}\left(S_{1}\right) \otimes \mathcal{F}_{d_{2}, L_{2}}^{r_{2}}\left(S_{2}\right)}
\end{aligned}
$$

und das Bild $F\left(\mathcal{F}_{d_{1}, L_{2}}^{r_{1}}\left(S_{1}\right) \otimes \mathcal{F}_{d_{2}, L_{2}}^{r_{2}}\left(S_{2}\right)\right)$ ist enthalten in $\mathcal{F}_{d, L_{2}}^{r}\left(S_{1} \times S_{2}\right)$. Die Abbildung ist wohldefiniert, denn ist $z=\sum_{j=1}^{n} x_{j} \otimes y_{j} \in \mathcal{F}_{d_{1}, L_{2}}^{r_{1}}\left(S_{1}\right) \otimes \mathcal{F}_{d_{2}, L_{2}}^{r_{2}}\left(S_{2}\right)$ eine Darstellung der Null in diesem Raum, so folgern wir für jedes $\tilde{z} \in \mathcal{F}_{d_{1}, L_{2}}^{r_{1}}\left(S_{1}\right) \otimes \mathcal{F}_{d_{2}, L_{2}}^{r_{2}}\left(S_{2}\right)$ die Gleichung $z+\tilde{z}=\tilde{z}$, damit gilt

$$
\begin{aligned}
\|\tilde{z}\|_{\mathcal{F}_{d_{1}, L_{2}}^{r_{1}}\left(S_{1}\right) \otimes \mathcal{F}_{d_{2}, L_{2}}^{r_{2}}\left(S_{2}\right)}^{2} & =(\tilde{z}, \tilde{z})_{\mathcal{F}_{d_{1}, L_{2}}^{r_{1}}\left(S_{1}\right) \otimes \mathcal{F}_{d_{2}, L_{2}}^{r_{2}}\left(S_{2}\right)} \\
& =(z+\tilde{z}, \tilde{z})_{\mathcal{F}_{d_{1}, L_{2}}^{r_{1}}}\left(S_{1}\right) \otimes \mathcal{F}_{d_{2}, L_{2}}^{r_{2}}\left(S_{2}\right) \\
& =(z, \tilde{z})_{\mathcal{F}_{d_{1}, L_{2}}^{r_{1}}\left(S_{1}\right) \otimes \mathcal{F}_{d_{2}, L_{2}}^{r_{2}}\left(S_{2}\right)}+\|\tilde{z}\|_{\mathcal{F}_{d_{1}, L_{2}}^{r_{1}}}^{2}\left(S_{1}\right) \otimes \mathcal{F}_{d_{2}, L_{2}}^{r_{2}}\left(S_{2}\right)
\end{aligned}
$$


und schließlich folgt

$$
0=(z, z)_{\mathcal{F}_{d_{1}, L_{2}}^{r_{1}}\left(S_{1}\right) \otimes \mathcal{F}_{d_{2}, L_{2}}^{r_{2}}\left(S_{2}\right)}=\|z\|_{\mathcal{F}_{d_{1}, L_{2}}^{r_{1}}\left(S_{1}\right) \otimes \mathcal{F}_{d_{2}, L_{2}}^{r_{2}}\left(S_{2}\right)}^{2}=\left\|f_{z}\right\|_{\mathcal{F}_{d_{1}+d_{2}, L_{2}}^{r_{2}}} .
$$

$F$ ist also eine Isometrie, und der Abschluß $\overline{F\left(\mathcal{F}_{d_{1}, L_{2}}^{r_{1}}\left(S_{1}\right) \otimes \mathcal{F}_{d_{2}, L_{2}}^{r_{2}}\left(S_{2}\right)\right)}$ ist die Vervollständigung des Raumes $\mathcal{F}_{d_{1}, L_{2}}^{r_{1}}\left(S_{1}\right) \otimes \mathcal{F}_{d_{2}, L_{2}}^{r_{2}}\left(S_{2}\right)$ bezüglich seiner Norm $\|\cdot\|_{\beta, \mathcal{F}_{d_{1}, L_{2}}^{r_{1}}\left(S_{1}\right) \otimes \mathcal{F}_{d_{2}, L_{2}}^{r_{2}}\left(S_{2}\right)}$. Da der Abschluß eines metrischen Raumes der Menge aller Grenzwerte konvergenter Folgen aus diesem Raum entspricht, folgern wir aus der Linearität von $F$ die Inklusion

$$
\overline{F\left(\mathcal{F}_{d_{1}, L_{2}}^{r_{1}}\left(S_{1}\right) \otimes \mathcal{F}_{d_{2}, L_{2}}^{r_{2}}\left(S_{2}\right)\right)} \subseteq \mathcal{F}_{d, L_{2}}^{r}\left(S_{1} \times S_{2}\right)
$$

es gilt also

$$
\left.\mathcal{F}_{d, L_{2}}^{r}\left(S_{1} \times S_{2}\right)=\overline{F\left(\mathcal{F}_{d_{1}, L_{2}}^{r_{1}}\left(S_{1}\right) \otimes \mathcal{F}_{d_{2}, L_{2}}^{r_{2}}\left(S_{2}\right)\right)} \quad \perp \quad \overline{F\left(\mathcal{F}_{d_{1}, L_{2}}^{r_{1}}\left(S_{1}\right) \otimes \mathcal{F}_{d_{2}, L_{2}}^{r_{2}}\left(S_{2}\right)\right.}\right)^{\perp} .
$$

Für $f \in{\overline{F\left(\mathcal{F}_{d_{1}, L_{2}}^{r_{1}}\left(S_{1}\right) \otimes \mathcal{F}_{d_{2}, L_{2}}^{r_{2}}\left(S_{2}\right)\right)}}^{\perp}$ folgern wir aufgrund des Satzes von Fubini mit $x \in \mathcal{F}_{d_{1}, L_{2}}^{r_{1}}\left(S_{1}\right), y \in \mathcal{F}_{d_{2}, L_{2}}^{r_{2}}\left(S_{2}\right)$

$$
\begin{aligned}
& 0=(x \otimes y, f)_{\mathcal{F}_{d, L_{2}}^{r}\left(S_{1} \times S_{2}\right)} \\
& =\sup _{\substack{\alpha=\left(\alpha_{1}, \ldots, \alpha_{d}\right) \in \mathbb{N}_{0}^{d} \\
\alpha_{j} \in\left\{0, \ldots, r T_{j}\right\}}} \int_{S_{1}} \int_{S_{2}}\left(D^{\alpha} x\left(s_{1}\right) y\left(s_{2}\right)\right)\left(D^{\alpha} f\right)\left(s_{1}, s_{2}\right) d s_{2} d s_{1} \\
& =\sup _{\substack{\tilde{\alpha}_{1}=\left(\alpha_{1}, \ldots, \alpha_{d_{1}}\right) \in N_{0}^{d_{1}} \\
\alpha_{j} \in\left\{0, \ldots, r T_{j}\right\}}} \int_{S_{1}} D_{s_{1}}^{\tilde{\alpha_{1}}} x\left(s_{1}\right) D_{s_{1}}^{\alpha_{1}} \sup _{\substack{\tilde{\alpha}_{2}=\left(\alpha_{d_{1}+1}, \ldots, \alpha_{d}\right) \in N_{0}^{d_{2}} \\
\alpha_{j} \in\left\{0, \ldots, r e_{j}\right\}}} \int_{S_{2}} D^{\tilde{\alpha}_{2}} y\left(s_{2}\right) D^{\tilde{\alpha}_{2}} f\left(s_{1}, s_{2}\right) d s_{2} d s_{1}
\end{aligned}
$$

und damit

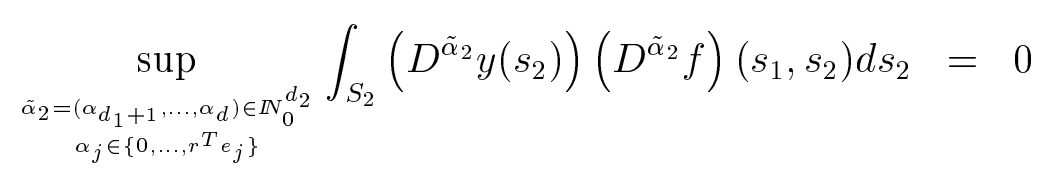

für fast alle $s_{1}$ und genauso $f\left(s_{1}, s_{2}\right)=0$ fast überall in $S_{1} \times S_{2}$.

Bemerkung 4.15 Aus Gründen der Normäquivalenz können wir auch

$$
\mathcal{F}_{d, L_{2}}^{r}(S)=\left\{f \in L_{2}(S):\|f\|_{\mathcal{F}_{d, L_{2}}^{r}(S), 2}<\infty\right\}
$$


mit

$$
\|f\|_{\mathcal{F}_{d, L_{2}}^{r}(S), 2}=\left(\sum_{\substack{\alpha=\left(\alpha_{1}, \ldots, \alpha_{d}\right) \in \mathbb{N}_{0}^{d} \\ \alpha_{j} \in\left\{0, \ldots, r_{j}\right\}}}\left\|D^{\alpha} f\right\|_{L_{2}(S)}^{2}\right)^{\frac{1}{2}}
$$

schreiben.

Ähnliches folgern wir unter anderem für das Tensorprodukt von klassischen eindimensionalen Sobolev-Räumen.

Definition 4.16 Für $S \subseteq \mathbb{R}^{d}$ und $r \in \mathbb{N}_{0}^{d}$ gilt

$$
\mathcal{W}_{d, L_{p}}^{r}(S):=\left\{f \in L_{p}(S):\|f\|_{\mathcal{W}_{d, L_{p}}^{r}(S)}<\infty\right\}
$$

mit

$$
\|f\|_{\mathcal{W}_{d, L_{p}}^{r}(S)}=\left(\|f\|_{L_{p}(S)}^{2}+\left\|D^{r_{1}, \ldots, r_{\tilde{d}}} f\right\|_{L_{p}(S)}^{2}\right)^{\frac{1}{2}}
$$

Damit gilt:

Satz 4.17 Für aus abzählbaren meßbaren Mengen bestehende $S_{j} \subseteq \mathbb{R}^{d_{j}}, 1 \leq j \leq \tilde{d}$, und $r=\left(r_{1}, \ldots, r_{\tilde{d}}\right)$ ist die Isomorphie

$$
\mathcal{W}_{d_{1}, L_{2}}^{r_{1}}\left(S_{1}\right) \otimes_{\beta} \ldots \otimes_{\beta} \mathcal{W}_{d_{\tilde{d}}, L_{2}}^{r_{\tilde{d}}}\left(S_{\tilde{d}}\right) \simeq \mathcal{W}_{d_{1}+\ldots+d_{\tilde{d}}, L_{2}}^{r}\left(S_{1} \times \ldots \times S_{\tilde{d}}\right)
$$

erfüllt.

Der Satz kann in völliger Analogie zum Beweis von Satz 4.14 geführt werden.

Der folgende Satz ist ein Resultat aus [18]. Er verwendet den Begriff des Atoms in einem meßbaren Raum, welcher eine meßbare Menge $A$ mit positivem Maß definiert, wobei jede meßbare Untermenge entweder das Maß 0 oder das von $A$ besitzt.

Satz 4.18 Seien $S_{1}, \ldots, S_{d}$ endliche meßbare atomfreie Räume. Dann gilt für $1 \leq p<\infty$

$$
L_{p}\left(S_{1}\right) \otimes_{\alpha_{p}} \ldots \otimes_{\alpha_{p}} L_{p}\left(S_{d}\right) \simeq L_{p}\left(S_{1} \times \ldots \times S_{d}\right)
$$


Da Lebesgue-meßbare Räume atomfrei sind, kann der Satz dort angewandt werden. Im Fall $p=\infty$ wird im allgemeinen keine Gleichheit erlangt; dort ist der Tensorproduktraum lediglich im Raum $L_{\infty}\left(S_{1} \times \ldots \times S_{d}\right)$ enthalten. Wir verallgemeinern:

Satz 4.19 Sind $S_{1}, \ldots, S_{\tilde{d}}$ endliche meßbare atomfreie Räume, so gilt für $1 \leq p<\infty$, $1 \leq j \leq \tilde{d}$ und $r=\left(r_{1}, \ldots, r_{\tilde{d}}\right)$

$$
\mathcal{F}_{d_{1}, L_{p}}^{r_{1}}\left(S_{1}\right) \otimes_{\alpha_{p}} \ldots \otimes_{\alpha_{p}} \mathcal{F}_{d_{\tilde{d}}, L_{p}}^{r_{\tilde{d}}}\left(S_{\tilde{d}}\right) \simeq \mathcal{F}_{d_{1}+\ldots+d_{\tilde{d}}, L_{p}}^{r}\left(S_{1} \times \ldots \times S_{\tilde{d}}\right)
$$

Um den Satz beweisen zu können, formulieren wir zunächst

Definition 4.20 Sei $S$ meßbar. Mit $\mathcal{F}_{d, L_{p}}^{r}(S, Y)$ sei für $d \in \mathbb{N}$ und $r=\left(r_{1}, \ldots, r_{d}\right)$ der Raum der Funktionen $f: S \rightarrow Y$ bezeichnet, für die

$$
\|f\|_{\mathcal{F}_{d, L_{p}}^{r}(S, Y)}=\sup _{\substack{\alpha=\left(\alpha_{1}, \ldots, \alpha_{d}\right) \in \mathbb{N}_{0}^{d} \\ \alpha_{l} \in\left\{0, \ldots, r_{l}\right\}}}\left(\int\left\|D^{\alpha} f(s)\right\|_{Y}^{p} d s\right)^{\frac{1}{p}}<\infty
$$

gilt.

Satz 4.21 Sei $S$ ein endlicher meßbarer atomfreier Raum, Y sei ein Banachraum mit

$$
\mathcal{F}_{d, L_{p}}^{r}(S) \otimes_{\alpha_{p}} Y \simeq Y \otimes_{\alpha_{p}} \mathcal{F}_{d, L_{p}}^{r}(S)
$$

Dann folgt für $1 \leq p<\infty$

$$
\mathcal{F}_{d, L_{p}}^{r}(S) \otimes_{\alpha_{p}} Y \simeq \mathcal{F}_{d, L_{p}}^{r}(S, Y)
$$

Beweis von Satz 4.21: Grundlage dieses Beweises ist die Verwendung von Elementarfunktionen, d.h. von meßbaren Funktionen, die nur endlich viele Werte annehmen, und ihrer Dichtheit in den $L_{p}$-Räumen für $1 \leq p<\infty$, vgl. [4]. Nach [18] gibt es solche Elementarfunktionen $\tilde{f}_{1}, \ldots, \tilde{f}_{n} \in L_{p}(S)$ mit $\left\|\tilde{f}_{j}-f_{j}\right\|<\epsilon$ zu gegebenen $f_{1}, \ldots, f_{n} \in L_{p}(S), \epsilon>0$ und

$$
\tilde{f}_{j} \in \operatorname{span}\left\{g_{1}, \ldots, g_{m}\right\}
$$

mit $g_{j} \cdot g_{k}=0$ für $j \neq k$ und $\sum_{k=1}^{m} g_{k}=1$. Die Funktionen $g_{j}$ bestehen dabei aus Produkten von charakteristischen Funktionen. Aus der Abgeschlossenheit der schwachen Ableitungsabbildung folgern wir die Dichtheit der Elementarfunktionen aus $\mathcal{F}_{d, L_{p}}^{r}(S)$ im selben Raum. Damit existieren zu Funktionen $f_{1}, \ldots, f_{n} \in \mathcal{F}_{d, L_{p}}^{r}(S)$ 
Elementarfunktionen $\tilde{f}_{1}, \ldots, \tilde{f}_{n} \in \mathcal{F}_{d, L_{p}}^{r}(S)$ mit den oben genannten Eigenschaften. Weiterhin benutzen wir im Beweis, daß die Normen der Einbettungsoperatoren

$$
A: \mathcal{F}_{d, L_{p}}^{r}(S, Y) \rightarrow Y \otimes_{\alpha_{p}} \mathcal{F}_{d, L_{p}}^{r}(S)
$$

und

$$
B: \mathcal{F}_{d, L_{p}}^{r}(S) \otimes_{\alpha_{p}} Y \rightarrow \mathcal{F}_{d, L_{p}}^{r}(S, Y)
$$

den Wert 1 besitzen, was im folgenden noch zu zeigen ist. Bezeichnen wir die Isometrie zwischen $\mathcal{F}_{d, L_{p}}^{r}(S) \otimes_{\alpha_{p}} Y$ und $Y \otimes_{\alpha_{p}} \mathcal{F}_{d, L_{p}}^{r}(S)$ mit $F$, so folgern wir für jede Elementarfunktion $f \in \mathcal{F}_{d, L_{p}}^{r}(S, Y)$ der Gestalt $f(s)=\sum_{j=1}^{n} g_{j}(s) y_{j}$

$$
\begin{aligned}
(B \circ F \circ A) f(s) & =(B \circ F \circ A) \sum_{j=1}^{n} g_{j}(s) y_{j} \\
& =(B \circ F) \sum_{j=1}^{n} y_{j} \otimes g_{j} \\
& =B \sum_{j=1}^{n} g_{j} \otimes y_{j} \\
& =\sum_{j=1}^{n} g_{j}(s) y_{j} \\
& =f(s) .
\end{aligned}
$$

Wegen $\|A\|_{\mathcal{F}_{d, L_{p}}^{r}(S, Y), Y \otimes_{\alpha_{p}} \mathcal{F}_{d, L_{p}}^{r}(S)}=\|B\|_{\mathcal{F}_{d, L_{p}}^{r}(S) \otimes_{\alpha_{p}} Y, \mathcal{F}_{d, L_{p}}^{r}(S, Y)}=1$ folgern wir

$$
\|f\|_{\mathcal{F}_{d, L_{p}}^{r}(S, Y)} \geq\|A f\|_{Y \otimes_{\alpha_{p}} \mathcal{F}_{d, L_{p}}^{r}(S)}
$$

und

$$
\|(F \circ A) f\|_{\mathcal{F}_{d, L_{p}}^{r}(S) \otimes_{\alpha_{p}} Y} \geq\|(B \circ F \circ A) f\|_{\mathcal{F}_{d, L_{p}}^{r}(S, Y)}=\|f\|_{\mathcal{F}_{d, L_{p}}^{r}(S, Y)}
$$

und damit

$$
\begin{aligned}
\|f\|_{\mathcal{F}_{d, L_{p}}^{r}(S, Y)} & \geq\|A f\|_{Y \otimes_{\alpha_{p}} \mathcal{F}_{d, L_{p}}^{r}(S)} \\
& =\|F\|_{\mathcal{F}_{d, L_{p}}^{r}(S) \otimes_{\alpha_{p}} Y, Y \otimes_{\alpha_{p}} \mathcal{F}_{d, L_{p}}^{r}(S)}\|A f\|_{Y \otimes_{\alpha_{p}} \mathcal{F}_{d, L_{p}}^{r}(S)} \\
& \geq\|(F \circ A) f\|_{\mathcal{F}_{d, L_{p}}^{r}(S) \otimes_{\alpha_{p}} Y} \\
& \geq\|f\|_{\mathcal{F}_{d, L_{p}}^{r}(S, Y) .}
\end{aligned}
$$

Es folgt $\|(F \circ A) f\|_{\mathcal{F}_{d, L_{p}}^{r}(S) \otimes_{\alpha_{p}} Y}=\|f\|_{\mathcal{F}_{d, L_{p}}^{r}(S, Y)} ; F \circ A$ ist also eine Isometrie und $\mathcal{F}_{d, L_{p}}^{r}(S, Y)$ die Vervollständigung von $\mathcal{F}_{d, L_{p}}^{r}(S) \otimes Y$ unter der $\alpha_{p}$-Norm. 
Es bleibt noch zu zeigen, daß die Normen von $A$ und $B$ den Wert 1 haben. Im Fall von $A$ wählen wir eine Elementarfunktion aus $\mathcal{F}_{d, L_{p}}^{r}(S, Y)$, die wir in der Form

$$
f(s)=\sum_{j=1}^{n} g_{j}(s) y_{j}
$$

mit $y_{j} \in Y$ und $g_{j} \cdot g_{k}=0$ für $j \neq k$ darstellen können. Da die Funktionen $g_{j}$ Produkte von charakteristischen Funktionen sind, gilt $g_{j} \neq 0$ und wir können $\left\|g_{j}\right\|_{\mathcal{F}_{d, L_{p}}^{r}(S)}=1$ annehmen. Wir wollen nun zeigen, daß

$$
\|A f\|_{\alpha_{p}, Y \otimes \mathcal{F}_{d, L_{p}}^{r}(S)} \leq\|f\|_{\mathcal{F}_{d, L_{p}}^{r}(S, Y)}
$$

erfüllt ist. Nach [18] gilt mit $\frac{1}{p}+\frac{1}{q}=1$

$$
\sup _{\substack{\psi \in X^{*} \\\|\psi\|_{X^{*}=1}}}\left(\sum_{j=1}^{n}\left|\psi\left(g_{j}\right)\right|^{q}\right)^{\frac{1}{q}}=\sup _{\substack{\sum_{j=1}^{n}\left|\lambda_{j}\right|^{p}=1 \\\left(\lambda_{1}, \ldots, \lambda_{n}\right) \in \mathbb{R}^{n}}}\left\|\sum_{j=1}^{n} \lambda_{j} g_{j}\right\|_{X}
$$

für jeden Banachraum $X$, und im Fall $g_{1}, \ldots, g_{n} \in L_{p}(S)$ nimmt dieser Ausdruck den Wert $\max _{1 \leq j \leq n}\left\|g_{j}\right\|_{L_{p}(S)}$ an. Daraus folgt

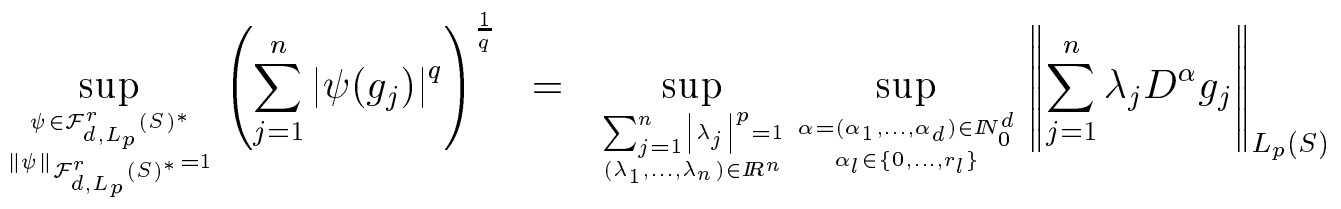

$$
\begin{aligned}
& =\sup _{\substack{\alpha=\left(\alpha_{1}, \ldots, \alpha_{d}\right) \in N_{0}^{d} \\
\alpha_{l} \in\left\{0, \ldots, r_{l}\right\}}} \max _{1 \leq j \leq n}\left\|D^{\alpha} g_{j}\right\|_{L_{p}(S)} \\
& =\max _{1 \leq j \leq n}\left\|g_{j}\right\|_{\mathcal{F}_{d, L_{p}}^{r}(S)} \\
& =1 \text {. }
\end{aligned}
$$

Damit gilt

$$
\begin{aligned}
\|A f\|_{\alpha_{p}, Y \otimes \mathcal{F}_{d, L_{p}}^{r}(S)} & =\inf _{A f=\sum_{j=1}^{n} y_{j} \otimes g_{j}}\left(\sum_{j=1}^{n}\left\|y_{j}\right\|_{Y}^{p}\right)^{\frac{1}{p}} \\
& \leq\left(\sum_{j=1}^{n}\left\|y_{j}\right\|_{Y}^{p}\right)^{\frac{1}{p}} \\
& =\left(\sum_{j=1}^{n}\left\|y_{j}\right\|_{Y}^{p}\right)^{\frac{1}{p}}\left\|g_{j}\right\|_{\mathcal{F}_{d, L_{p}}^{r}(S)}
\end{aligned}
$$




$$
\begin{aligned}
& =\left(\sum_{j=1}^{n}\left\|y_{j}\right\|_{Y}^{p}\left\|g_{j}\right\|_{\mathcal{F}_{d, L_{p}}^{r}(S)}^{p}\right)^{\frac{1}{p}} \\
& =\left(\sum_{j=1}^{n}\left\|y_{j}\right\|_{Y}^{p} \sup _{\substack{\alpha=\left(\alpha_{1}, \ldots, \alpha_{d}\right) \in \mathbb{N}_{0}^{d} \\
\alpha_{l} \in\left\{0, \ldots, r_{l}\right\}}} \int_{S}\left|D^{\alpha} g_{j}(s)\right|^{p} d s\right)^{\frac{1}{p}} \\
& =\sup _{\substack{\alpha=\left(\alpha_{1}, \ldots, \alpha_{d}\right) \in \mathbb{N}_{0}^{d} \\
\alpha_{l} \in\left\{0, \ldots, r_{l}\right\}}}\left(\int_{S} \sum_{j=1}^{n}\left\|y_{j} D^{\alpha} g_{j}(s)\right\|_{Y}^{p} d s\right)^{\frac{1}{p}} \\
& =\sup _{\substack{\alpha=\left(\alpha_{1}, \ldots, \alpha_{d}\right) \in \mathbb{N}_{0}^{d} \\
\alpha_{l} \in\left\{0, \ldots, r_{l}\right\}}}\left(\sum_{k=1}^{n} \int_{\left\{t: D^{\alpha} g_{k}(t) \neq 0\right\}} \sum_{j=1}^{n}\left\|y_{j} D^{\alpha} g_{j}(s)\right\|_{Y}^{p} d s\right)^{\frac{1}{p}} \\
& =\sup _{\substack{\alpha=\left(\alpha_{1}, \ldots, \alpha_{d}\right) \in \mathbb{N}_{0}^{d} \\
\alpha_{l} \in\left\{0, \ldots, r_{l}\right\}}}\left(\sum_{k=1}^{n} \int_{\left\{t: D^{\alpha} g_{k}(t) \neq 0\right\}}\left\|y_{k} D^{\alpha} g_{k}(s)\right\|_{Y}^{p} d s\right)^{\frac{1}{p}} \\
& =\sup _{\substack{\alpha=\left(\alpha_{1}, \ldots, \alpha_{d}\right) \in \mathbb{N}_{0}^{d} \\
\alpha_{l} \in\left\{0, \ldots, r_{l}\right\}}}\left(\int_{S}\left\|\sum_{k=1}^{n} y_{k} D^{\alpha} g_{k}(s)\right\|_{Y}^{p} d s\right)^{\frac{1}{p}} \\
& =\|f\|_{\mathcal{F}_{d, L_{p}}^{r}(S, Y)} .
\end{aligned}
$$

Für $f_{0}(s)=c \cdot y$ mit der auf $S$ konstanten Funktion $c$, wobei $\|c\|_{\mathcal{F}_{d, L_{p}}^{r}(S)}=1$ und $y \in Y$ erfüllt seien, gilt wegen (19)

$$
\left\|f_{0}\right\|_{\mathcal{F}_{d, L_{p}}^{r}(S, Y)}=\|y\|_{Y}=\left\|A f_{0}\right\|_{\alpha_{p}, Y \otimes \mathcal{F}_{d, L p}^{r}(S)}
$$

und wir folgern $\|A\|_{\mathcal{F}_{d, L p}^{r}(S, Y), \mathcal{F}_{d, L p}^{r}(S)}=1$.

Im Fall $B$ schreiben wir $B z(s)=\sum_{j=1}^{n} g_{j}(s) y_{j}=: f_{z}(s)$ für ein Element $z=\sum_{j=1}^{n} g_{j} \otimes y_{j}$ aus $\mathcal{F}_{d, L_{p}}^{r}(S) \otimes Y$. Es gilt für $2 \leq q<\infty$

$$
\begin{aligned}
\left\|f_{z}\right\|_{\mathcal{F}_{d, L_{p}}^{r}(S, Y)} & =\sup _{\substack{\alpha=\left(\alpha_{1}, \ldots, \alpha_{d}\right) \in N_{N}^{d} \\
\alpha_{l} \in\left\{0, \ldots, r_{l}\right\}}}\left(\int_{S}\left\|\sum_{j=1}^{n} y_{j} D^{\alpha} g_{j}(s)\right\|_{Y}^{p} d s\right)^{\frac{1}{p}} \\
& =\sup _{\substack{\alpha=\left(\alpha_{1}, \ldots, \alpha_{d}\right) \in N_{N}^{d} \\
\alpha_{l} \in\left\{0, \ldots, r_{l}\right\}}}\left(\int_{S} \sup _{\substack{\psi \in Y^{*} \\
\|\|_{Y^{*}=1}}}\left|\sum_{j=1}^{n} \psi\left(y_{j}\right) D^{\alpha} g_{j}(s)\right|^{p} d s\right)^{\frac{1}{p}} \\
& \leq \sum_{j=1}^{n}\left\|g_{j}\right\|_{\mathcal{F}_{d, L_{p}}^{r}(S)} \sup _{\substack{\psi \in Y^{*} \\
\|\psi\|_{Y^{*}=1}}}\left(\sum_{k=1}^{n}\left|\psi\left(y_{k}\right)\right|^{q}\right)^{\frac{1}{q}}
\end{aligned}
$$


und genauso

$$
\left\|f_{z}\right\|_{\mathcal{F}_{d, L_{1}}^{r}(S, Y)} \leq \sum_{j=1}^{n}\left\|g_{j}\right\|_{\mathcal{F}_{d, L_{1}}^{r}(S)} \sup _{\substack{\psi \in Y^{*} \\\|\psi\|_{Y^{*}=1}}}\left(\max _{1 \leq k \leq n}\left|\psi\left(y_{k}\right)\right|\right) .
$$

Damit folgt

$$
\left\|f_{z}\right\|_{\mathcal{F}_{d, L_{p}}^{r}(S, Y)} \leq\|z\|_{\alpha_{p}, \mathcal{F}_{d, L_{p}}^{r}(S) \otimes Y}
$$

und für $z_{0}=g \otimes y$ mit $g \in \mathcal{F}_{d, L_{p}}^{r}(S)$ und $y \in Y$ die Gleichheit $\left\|f_{z_{0}}\right\|_{\mathcal{F}_{d, L_{p}}^{r}(S, Y)}=\|g\|_{\mathcal{F}_{d, L_{p}}^{r}(S)}\|y\|_{Y}$.

Beweis von Satz 4.19: Um Satz 4.21 anwenden zu können, müssen wir zeigen, daß die Räume $\mathcal{F}_{d_{1}, L_{p}}^{r_{1}}\left(S_{1}\right) \otimes \mathcal{F}_{d_{2}, L_{p}}^{r_{2}}\left(S_{2}\right)$ und $\mathcal{F}_{d_{2}, L_{p}}^{r_{2}}\left(S_{2}\right) \otimes \mathcal{F}_{d_{1}, L_{p}}^{r_{1}}\left(S_{1}\right)$ isometrisch sind. Die Abbildung

$$
F: \mathcal{F}_{d_{1}, L_{p}}^{r_{1}}\left(S_{1}\right) \otimes \mathcal{F}_{d_{2}, L_{p}}^{r_{2}}\left(S_{2}\right) \rightarrow \mathcal{F}_{d_{2}, L_{p}}^{r_{2}}\left(S_{2}\right) \otimes \mathcal{F}_{d_{1}, L_{p}}^{r_{1}}\left(S_{1}\right)
$$

mit $F(z)=\tilde{z}$ für $z=\sum_{j=1}^{n} f_{1 j} \otimes f_{2 j}, \tilde{z}=\sum_{j=1}^{n} f_{2 j} \otimes f_{1 j}$ und $f_{i j} \in \mathcal{F}_{d_{i}, L_{p}}^{r_{i}}\left(S_{i}\right)$ für $i=1,2$ erfüllt diese Bedingung, denn nennen wir die Funktion

$$
f\left(s_{1}\right)=\sum_{j=1}^{n} f_{1 j}\left(s_{1}\right) f_{2 j} \in \mathcal{F}_{d_{1}, L_{p}}^{r_{1}}\left(S_{1}, \mathcal{F}_{d_{2}, L_{p}}^{r_{2}}\left(S_{2}\right)\right),
$$

so folgern wir mit (17) und (18)

$$
\|z\|_{\mathcal{F}_{d_{1}, L_{p}}^{r_{1}}\left(S_{1}\right) \otimes \mathcal{F}_{d_{2}, L_{p}}^{r_{2}}\left(S_{2}\right)} \leq\|f\|_{\mathcal{F}_{d_{1}, L_{p}}^{r_{1}}\left(S_{1}, \mathcal{F}_{d_{2}, L_{p}}^{r_{2}}\left(S_{2}\right)\right)} \leq\|F(z)\|_{\mathcal{F}_{d_{2}, L_{p}}^{r_{2}}\left(S_{2}\right) \otimes \mathcal{F}_{d_{1}, L_{p}}^{r_{1}}\left(S_{1}\right)}
$$

und aus Symmetriegründen die Behauptung. Nun muß noch bewiesen werden, daß auch die Räume $\mathcal{F}_{d_{1}+d_{2}, L_{p}}^{r_{1}, r_{2}}\left(S_{1} \times S_{2}\right)$ und $\mathcal{F}_{d_{1}, L_{p}}^{r_{1}}\left(S_{1}, \mathcal{F}_{d_{2}, L_{p}}^{r_{2}}\left(S_{2}\right)\right)$ isometrisch sind. Dazu definieren wir eine Abbildung $\tilde{f} \rightarrow f$, wobei

$$
\tilde{f}\left(s_{1}, s_{2}\right)=f\left(s_{1}\right)\left(s_{2}\right)=\sum_{j=1}^{n} f_{1 j}\left(s_{1}\right) f_{2 j}\left(s_{2}\right)
$$

benannt sei. Mit dem Satz von Fubini gilt

$$
\begin{aligned}
\|\tilde{f}\|_{\mathcal{F}_{d_{1}+d_{2}, L_{p}}^{r_{1}, r_{2}}\left(S_{1} \times S_{2}\right)} & =\sup _{\substack{\left(\alpha_{1}, \ldots, \alpha_{d}\right) \in \mathbb{N}_{0}^{d} \\
\alpha_{l} \in\left\{0, \ldots, r_{l}\right\}}}\left(\int_{S_{1} \times S_{2}}\left|D^{\alpha} \tilde{f}\left(s_{1}, s_{2}\right)\right|^{p} d\left(s_{1}, s_{2}\right)\right)^{\frac{1}{p}} \\
& =\sup _{\substack{\left(\alpha_{1}, \ldots, \alpha_{d_{1}}\right) \in \mathbb{N}_{0}^{d_{1}} \\
\alpha_{l} \in\left\{0, \ldots, r_{l}\right\}}}\left(\int_{S_{1}}\left\|D^{\alpha} f\left(s_{1}\right)\right\|_{\mathcal{F}_{d_{2}, L_{p}}^{r_{2}}\left(S_{2}\right)}^{p} d s_{1}\right)^{\frac{1}{p}} \\
& =\|f\|_{\mathcal{F}_{d_{1}, L_{p}}^{r_{1}, p_{p}}}\left(S_{1}, \mathcal{F}_{d_{2}, L_{p}}^{r_{2}}\left(S_{2}\right)\right.
\end{aligned}
$$


Genauso beweisen wir:

Satz 4.22 Sind $S_{1}, \ldots, S_{d}$ meßbare atomfreie Räume und gilt $r=\left(r_{1}, \ldots, r_{\tilde{d}}\right)$, so ist die Isomorphie

$$
\mathcal{W}_{d_{1}, L_{p}}^{r_{1}}\left(S_{1}\right) \otimes_{\beta} \ldots \otimes_{\beta} \mathcal{W}_{d_{\tilde{d}}, L_{p}}^{r_{\tilde{d}}}\left(S_{\tilde{d}}\right) \simeq \mathcal{W}_{d_{1}+\ldots+d_{\tilde{d}}, L_{p}}^{r}\left(S_{1} \times \ldots \times S_{\tilde{d}}\right)
$$

erfüllt.

\subsection{Untersuchung geeigneter Native Spaces}

In diesem Abschnitt werden Native Spaces in Hinblick auf ihre Anwendbarkeit bei der Interpolation mit Unterstützung des Smolyak-Algorithmus betrachtet. Dazu müssen wir vorbereitend noch ein anderes Ergebnis formulieren.

Satz 4.23 Seien $\phi_{j}$ für $1 \leq j \leq \tilde{d}$ stetige, bedingt positiv definite Funktionen der Ordnung $m_{j}$ auf $\Omega_{j}$. Jede Funktion $\phi_{j}$ besitze eine verallgemeinerte Fouriertransformierte $\hat{\phi}_{j}$ mit der zusätzlichen Eigenschaft, daß $\hat{\phi}_{j}\left(\omega_{j}\right)\left\|\omega_{j}\right\|^{s_{j}}$ für $s_{j} \leq 2 m_{j}$ mit $\omega_{j} \in \Omega_{j}$ um Null integrierbar ist. Dann ist die Funktion $\Phi=\phi_{1} \otimes \ldots \otimes \phi_{\tilde{d}}$ bedingt positiv definit der Ordnung $m=\sum_{j=1}^{\tilde{d}} m_{j}$.

Beweis: Wir benutzen Satz 2.4 von Iske. Dazu müssen wir zunächst überprüfen, ob $\Phi$ eine verallgemeinerte Fouriertransformierte $\hat{\Phi}$ besitzt. Für jede Funktion $\gamma \in \mathcal{S}_{d}$ müßte die Gleichung

$$
(\Phi, \hat{\gamma})_{L_{2}\left(\mathbb{R}^{d}\right)}=(\hat{\Phi}, \gamma)_{L_{2}\left(\mathbb{R}^{d}\right)}
$$

erfüllt sein. Aufgrund von Korollar 4.11 reicht es aus, Funktionen aus dem Tensorraum von passenden Schwartzschen Räumen $\mathrm{zu}$ betrachten. Es gilt also mit $\gamma=\sum_{j=1}^{n} \gamma_{1 j} \otimes \gamma_{2 j} \in \mathcal{S}_{d_{1}} \otimes \mathcal{S}_{d_{2}}$ und dem Satz von Fubini

$$
\begin{aligned}
(\Phi, \hat{\gamma})_{L_{2}\left(\mathbb{R}^{d}\right)} & =\left(\Phi, \sum_{j=1}^{n} \hat{\gamma}_{1 j} \otimes \hat{\gamma}_{2 j}\right)_{L_{2}\left(\mathbb{R}^{d}\right)} \\
& =\int_{\mathbb{R}^{d}} \Phi(x) \sum_{j=1}^{n} \hat{\gamma}_{1 j} \otimes \hat{\gamma}_{2 j}(x) d x \\
& =\int_{\mathbb{R}^{d_{1}}} \int_{\mathbb{R}^{d_{2}}} \phi_{1}\left(x_{1}\right) \phi_{2}\left(x_{2}\right) \sum_{j=1}^{n} \hat{\gamma}_{1 j}\left(x_{1}\right) \hat{\gamma}_{2 j}\left(x_{2}\right) d x
\end{aligned}
$$




$$
\begin{aligned}
& =\sum_{j=1}^{n} \int_{\mathbb{R}^{d_{1}}} \phi_{1}\left(x_{1}\right) \hat{\gamma}_{1 j}\left(x_{1}\right) d x_{1} \int_{\mathbb{R}^{d_{2}}} \phi_{2}\left(x_{2}\right) \hat{\gamma}_{2 j}\left(x_{2}\right) d x_{2} \\
& =\sum_{j=1}^{n} \int_{\mathbb{R}^{d_{1}}} \hat{\phi}_{1}\left(x_{1}\right) \gamma_{1 j}\left(x_{1}\right) d x_{1} \int_{\mathbb{R}^{d_{2}}} \hat{\phi}_{2}\left(x_{2}\right) \gamma_{2 j}\left(x_{2}\right) d x_{2} \\
& =\int_{\mathbb{R}^{d}} \hat{\phi}_{1} \otimes \hat{\phi}_{2}(x) \sum_{j=1}^{n} \gamma_{1 j} \otimes \gamma_{2 j}(x) d x \\
& =\left(\hat{\phi}_{1} \otimes \hat{\phi}_{2}, \gamma\right)_{L_{2}\left(\mathbb{R}^{d}\right)},
\end{aligned}
$$

und $\hat{\phi}_{1} \otimes \hat{\phi}_{2}$ ist die verallgemeinerte Fouriertransformierte von $\Phi$. Genauso ist also $\hat{\phi}_{1} \otimes \ldots \otimes \hat{\phi}_{\tilde{d}}$ die verallgemeinerte Fouriertransformierte von $\phi_{1} \otimes \ldots \otimes \phi_{\tilde{d}}$. Weiterhin muß $\hat{\Phi}(\omega)\|\omega\|^{s}$ für $s \leq 2 m$ um Null integrierbar sein. Wir betrachten dazu für ein beliebig kleines $\epsilon>0$ mit $s_{j} \leq 2 m_{j}$ und $s=\sum_{j=1}^{\tilde{d}} s_{j}$

$$
\begin{aligned}
& \int_{\left\|\omega_{1}\right\|=\ldots=\left\|\omega_{\tilde{d}}\right\|<\epsilon} \hat{\Phi}(\omega)\|\omega\|^{s} d \omega \\
& =\int_{\left\|\omega_{1}\right\|=\ldots=\left\|\omega_{\tilde{d}}\right\|<\epsilon} \hat{\phi}_{1}\left(\omega_{1}\right) \cdot \ldots \cdot \hat{\phi}_{\tilde{d}}\left(\omega_{\tilde{d}}\right)\left(\left\|\omega_{1}\right\|^{2}+\ldots+\left\|\omega_{\tilde{d}}\right\|^{2}\right)^{\frac{s}{2}} d \omega \\
& =\int_{\left\|\omega_{1}\right\|=\ldots=\left\|\omega_{\tilde{d}}\right\|<\epsilon} \hat{\phi}_{1}\left(\omega_{1}\right) \cdot \ldots \cdot \hat{\phi}_{\tilde{d}}\left(\omega_{\tilde{d}}\right) \prod_{j=1}^{\tilde{d}}\left(\left\|\omega_{1}\right\|^{2}+\ldots+\left\|\omega_{\tilde{d}}\right\|^{2}\right)^{\frac{s_{j}}{2}} d \omega \\
& =\int_{\left\|\omega_{1}\right\|=\ldots=\left\|\omega_{\tilde{d}}\right\|<\epsilon} \hat{\phi}_{1}\left(\omega_{1}\right) \cdot \ldots \cdot \hat{\phi}_{\tilde{d}}\left(\omega_{\tilde{d}}\right) \tilde{d}^{\frac{s}{2}} \prod_{j=1}^{\tilde{d}}\left\|\omega_{j}\right\|^{s_{j}} d \omega \\
& =\tilde{d}^{\frac{s}{2}} \prod_{j=1}^{\tilde{d}} \int_{\left\|\omega_{j}\right\|<\epsilon} \hat{\phi}_{j}\left(\omega_{j}\right)\left\|\omega_{j}\right\|^{s_{j}} d \omega_{j} \\
& <\infty
\end{aligned}
$$

dabei wurde der Satz von Fubini benutzt. Wegen

$$
\int_{\|\omega\|<\delta}|\hat{\Phi}(\omega)|\|\omega\|^{s} d \omega \leq \int_{\left\|\omega_{1}\right\|=\ldots=\left\|\omega_{\hat{d}}\right\|<\epsilon}|\hat{\Phi}(\omega)|\|\omega\|^{s} d \omega
$$

bei passender Wahl von $\delta$ ist $\hat{\Phi}(\omega)\|\omega\|^{s}$ für $s \leq 2 m$ um Null integrierbar. Aus der bedingten positiven Definitheit der Funktionen $\phi_{j}$ schließen wir $\hat{\phi}_{j}\left(\omega_{j}\right) \geq 0$ für alle $\omega_{j} \neq 0$ und $\hat{\phi}_{j} \not \equiv 0$ und damit $\hat{\Phi}(\omega) \geq 0$ für alle $\omega \neq 0$ und $\hat{\Phi} \not \equiv 0$.

Nun steuern wir zunächst auf ein Ergebnis für positiv definite Funktionen zu.

Satz 4.24 Seien $H_{K_{1}}, \ldots, H_{K_{d}}$ reelle Hilberträume mit Kernfunktionen $K_{1}, \ldots, K_{d}$. Dann besitzt auch ihr Tensorraum bzw. seine Vervollständigung die Kernfunktion 
$K_{1} \otimes \ldots \otimes K_{d}$. Genauer gilt

$$
H_{K_{1}} \otimes_{\beta} \ldots \otimes_{\beta} H_{K_{d}} \simeq H_{K_{1} \otimes \ldots \otimes K_{d}} .
$$

Der Beweis findet sich bei [21]. Damit folgern wir

Satz 4.25 Seien $\mathcal{N}_{\phi_{j}}\left(\Omega_{j}\right)$ für $1 \leq j \leq \tilde{d}$ Native Spaces von stetigen, symmetrischen, positiv definiten Funktionen $\phi_{j}$, welche eine verallgemeinerte Fouriertransformierte besitzen. Dann gilt

$$
\mathcal{N}_{\phi_{1}}\left(\Omega_{1}\right) \otimes_{\beta} \ldots \otimes_{\beta} \mathcal{N}_{\phi_{\tilde{d}}}\left(\Omega_{\tilde{d}}\right) \simeq \mathcal{N}_{\phi_{1} \otimes \ldots \otimes \phi_{\tilde{d}}}\left(\Omega_{1} \times \ldots \times \Omega_{\tilde{d}}\right) .
$$

Der Beweis folgt sofort mit Satz 4.23. Für bedingt positiv definite Funktionen folgern wir

Satz 4.26 Es seien $\phi_{j}$ für $j=1,2$ bezüglich $\mathcal{P}_{j}$ bedingt positiv definit; ihre Tensorproduktfunktion $\phi_{1} \otimes \phi_{2}$ sei bedingt positiv definit bezüglich $\mathcal{P}_{1} \otimes \mathcal{P}_{2}$. Dann erfüllen ihre Native Spaces $\mathcal{N}_{\phi_{j}, \mathcal{P}_{j}}\left(\Omega_{j}\right)$ folgende Eigenschaften:

(1) $\mathcal{N}_{\phi_{1}, \mathcal{P}_{1}}\left(\Omega_{1}\right) \otimes \mathcal{N}_{\phi_{2}, \mathcal{P}_{2}}\left(\Omega_{2}\right)=\mathcal{F}_{\phi_{1}, \mathcal{P}_{1}}\left(\Omega_{1}\right) \otimes \mathcal{F}_{\phi_{2}, \mathcal{P}_{2}}\left(\Omega_{2}\right) \oplus \mathcal{P}_{\phi_{1}, \phi_{2}}\left(\Omega_{1} \times \Omega_{2}\right)$,

(2) $\mathcal{F}_{\phi_{1}, \mathcal{P}_{1}}\left(\Omega_{1}\right) \otimes_{\beta} \mathcal{F}_{\phi_{2}, \mathcal{P}_{2}}\left(\Omega_{2}\right) \subseteq \mathcal{F}_{\phi_{1} \otimes \phi_{2}, \mathcal{P}_{1} \otimes \mathcal{P}_{2}}\left(\Omega_{1} \times \Omega_{2}\right)$

mit

$$
\mathcal{P}_{\phi_{1}, \phi_{2}}\left(\Omega_{1} \times \Omega_{2}\right):=\left(\mathcal{P}_{1} \otimes \mathcal{F}_{\phi_{2}, \mathcal{P}_{2}}\left(\Omega_{2}\right)\right) \oplus\left(\mathcal{F}_{\phi_{1}, \mathcal{P}_{1}}\left(\Omega_{1}\right) \otimes \mathcal{P}_{2}\right) \oplus\left(\mathcal{P}_{1} \otimes \mathcal{P}_{2}\right)
$$

Beweis: Die Identität

$$
\mathcal{N}_{\phi_{1}, \mathcal{P}_{1}}\left(\Omega_{1}\right) \otimes \mathcal{N}_{\phi_{2}, \mathcal{P}_{2}}\left(\Omega_{2}\right)=\mathcal{F}_{\phi_{1}, \mathcal{P}_{1}}\left(\Omega_{1}\right) \otimes \mathcal{F}_{\phi_{2}, \mathcal{P}_{2}}\left(\Omega_{2}\right) \oplus \mathcal{P}_{\phi_{1}, \phi_{2}}\left(\Omega_{1} \times \Omega_{2}\right)
$$

ist offensichtlich, wenn man die Darstellung jedes Elementes

$$
\sum_{j=1}^{n} f_{1 j} \otimes f_{2 j} \in \mathcal{N}_{\phi_{1}, \mathcal{P}_{1}}\left(\Omega_{1}\right) \otimes \mathcal{N}_{\phi_{2}, \mathcal{P}_{2}}\left(\Omega_{2}\right)
$$

mit $f_{l j}=\Pi_{\mathcal{P}_{j}} f_{l j}+\left(I d-\Pi_{\mathcal{P}_{j}}\right) f_{l j}$ betrachtet. Für das Funktional

$$
\lambda=\sum_{j=1}^{n} \lambda_{X_{1 j}, M_{1 j}}^{1} \otimes \lambda_{X_{2 j}, M_{2 j}}^{2} \in L_{\mathcal{P}_{1}}\left(\Omega_{1}\right) \otimes L_{\mathcal{P}_{2}}\left(\Omega_{2}\right)
$$


mit $\lambda_{X_{l j}, M_{l j}}^{l} \in L_{\mathcal{P}_{l}}\left(\Omega_{l}\right)$ und den Punktesätzen $X_{l}=\left\{x_{l 1}, \ldots, x_{l M_{l}}\right\}$ folgern wir

$$
\lambda(f)=\sum_{j=1}^{n} \sum_{k_{1}=1}^{M_{1 j}} \sum_{k_{2}=1}^{M_{2 j}} \lambda_{1 k_{1}} \lambda_{2 k_{2}} f\left(x_{1 k_{1}}, x_{2 k_{2}}\right) \in L_{\mathcal{P}_{1} \otimes \mathcal{P}_{2}}\left(\Omega_{1} \times \Omega_{2}\right),
$$

da zusätzlich $\lambda\left(\mathcal{P}_{1} \otimes \mathcal{P}_{2}\right)=\{0\}$ erfüllt ist. Damit gilt auch

$$
L_{\mathcal{P}_{1}}\left(\Omega_{1}\right) \otimes_{\beta} L_{\mathcal{P}_{2}}\left(\Omega_{2}\right) \subseteq \mathcal{L}_{\Phi, \mathcal{P}}(\Omega)
$$

mit $\Phi:=\phi_{1} \otimes \phi_{2}, \mathcal{P}:=\mathcal{P}_{1} \otimes \mathcal{P}_{2}$ und $\Omega:=\Omega_{1} \times \Omega_{2}$. Wir bezeichnen das Skalarprodukt von $L_{\mathcal{P}_{1}}\left(\Omega_{1}\right) \otimes_{\beta} L_{\mathcal{P}_{2}}\left(\Omega_{2}\right)$ mit $(\cdot, \cdot)_{\phi_{1}, \phi_{2}, \beta}$. Es stimmt mit dem Skalarprodukt von $\mathcal{L}_{\Phi, \mathcal{P}}(\Omega)$ aufgrund von

$$
\begin{aligned}
(\lambda, \mu)_{\phi_{1}, \phi_{2}, \beta} & =\sum_{j=1}^{M} \sum_{k=1}^{N}\left(\lambda_{1 j}, \mu_{1 k}\right)_{\phi_{1}}\left(\lambda_{2 j}, \mu_{2 k}\right)_{\phi_{2}} \\
& =\sum_{j=1}^{M} \sum_{k=1}^{N} \lambda_{1 j}^{x_{1}} \mu_{1 k}^{y_{1}} \phi_{1}\left(x_{1}, y_{1}\right) \lambda_{2 j}^{x_{2}} \mu_{2 k}^{y_{2}} \phi_{2}\left(x_{2}, y_{2}\right) \\
& =\sum_{j=1}^{M} \sum_{k=1}^{N}\left(\lambda_{1 j} \otimes \lambda_{2 j}\right)^{x}\left(\mu_{1 k} \otimes \mu_{2 k}\right)^{y} \Phi(x, y) \\
& =\lambda^{x} \mu^{y} \Phi(x, y) \\
& =(\lambda, \mu)_{\Phi}
\end{aligned}
$$

mit $\lambda=\sum_{j=1}^{M} \lambda_{1 j} \otimes \lambda_{2 j}$ und $\mu=\sum_{k=1}^{N} \mu_{1 k} \otimes \mu_{2 k} \in L_{\mathcal{P}_{1}}\left(\Omega_{1}\right) \otimes_{\beta} L_{\mathcal{P}_{2}}\left(\Omega_{2}\right)$ überein. Wir wählen nun

$$
f=\sum_{j=1}^{n} f_{1 j} \otimes f_{2 j} \in \mathcal{F}_{\phi_{1}, \mathcal{P}_{1}}\left(\Omega_{1}\right) \otimes \mathcal{F}_{\phi_{2}, \mathcal{P}_{2}}\left(\Omega_{2}\right)
$$

mit $f_{l j} \in \mathcal{F}_{\phi_{l}, \mathcal{P}_{l}}\left(\Omega_{l}\right)$. Da zu $f_{l j}$ Funktionale $\lambda_{f_{l j}}$ aus $\mathcal{L}_{\phi_{l}, \mathcal{P}_{l}}\left(\Omega_{l}\right)$ existieren mit $R_{\phi_{l}, \Omega_{l}}\left(\lambda_{f_{l j}}\right)=f_{l j}$, schreiben wir für $x \in \Omega_{1} \times \Omega_{2}$

$$
\begin{aligned}
f(x) & =\sum_{j=1}^{n} R_{\phi_{1}, \Omega_{1}}\left(\lambda_{f_{1 j}}\right) \otimes R_{\phi_{2}, \Omega_{2}}\left(\lambda_{f_{2 j}}\right)(x) \\
& =\sum_{j=1}^{n}\left(\lambda_{f_{1 j}}, \delta_{\left(x_{1}\right)}\right)_{\phi_{1}}\left(\lambda_{f_{2 j}}, \delta_{\left(x_{2}\right)}\right)_{\phi_{2}} \\
& =\sum_{j=1}^{n} \lambda_{f_{1 j}}^{y_{1}} \delta_{\left(x_{1}\right)} \phi_{1}\left(x_{1}, y_{1}\right) \lambda_{f_{2 j}}^{y_{2}} \delta_{\left(x_{2}\right)} \phi_{2}\left(x_{2}, y_{2}\right) \\
& =\left(\sum_{j=1}^{n} \lambda_{f_{1 j}}^{y_{1}} \otimes \lambda_{f_{2 j}}^{y_{2}}\right)\left(\delta_{\left(x_{1}\right)} \otimes \delta_{\left(x_{2}\right)}\right) \phi_{1}\left(x_{1}, y_{1}\right) \phi_{2}\left(x_{2}, y_{2}\right)
\end{aligned}
$$




$$
\begin{aligned}
& =\left(\sum_{j=1}^{n} \lambda_{f_{1 j}} \otimes \lambda_{f_{2 j}}\right)^{y} \delta_{(x)} \Phi(x, y) \\
& =\left(\sum_{j=1}^{n} \lambda_{f_{1 j}} \otimes \lambda_{f_{2 j}}, \delta_{(x)}\right)_{\Phi} \\
& =R_{\Phi, \Omega}\left(\sum_{j=1}^{n} \lambda_{f_{1 j}} \otimes \lambda_{f_{2 j}}\right) ;
\end{aligned}
$$

damit gilt $f \in \mathcal{F}_{\Phi, \mathcal{P}}(\Omega)$ und

$$
\mathcal{F}_{\phi_{1}, \mathcal{P}_{1}}\left(\Omega_{1}\right) \otimes \mathcal{F}_{\phi_{2}, \mathcal{P}_{2}}\left(\Omega_{2}\right) \subseteq R_{\Phi, \Omega}\left(L_{\mathcal{P}}(\Omega)\right)
$$

Wegen

$$
\|f\|_{\phi_{1}, \phi_{2}, \beta}^{2}=(f, f)_{\phi_{1}, \phi_{2}, \beta}=\left(\lambda_{f}, \lambda_{f}\right)_{\phi_{1}, \phi_{2}, \beta}=\left(\lambda_{f}, \lambda_{f}\right)_{\Phi}=\|f\|_{\Phi}^{2}
$$

mit $\lambda_{f}=\sum_{j=1}^{n} \lambda_{f_{1 j}} \otimes \lambda_{f_{2 j}}$ folgern wir $\mathcal{F}_{\phi_{1}, \mathcal{P}_{1}}\left(\Omega_{1}\right) \otimes_{\beta} \mathcal{F}_{\phi_{2}, \mathcal{P}_{2}}\left(\Omega_{2}\right) \subseteq \mathcal{F}_{\Phi, \mathcal{P}}(\Omega)$.

Ein Analogon kann offensichtlich auch für $d$-variate Tensorprodukte formuliert werden.

Wir wählen folgende

Definition 4.27 Wir nennen den Raum

$$
\mathcal{N}_{\phi_{1}, \phi_{2}, \mathcal{P}_{1}, \mathcal{P}_{2}}^{\otimes}\left(\Omega_{1} \times \Omega_{2}\right):=\mathcal{F}_{\phi_{1}, \mathcal{P}_{1}}\left(\Omega_{1}\right) \otimes_{\beta} \mathcal{F}_{\phi_{2}, \mathcal{P}_{2}}\left(\Omega_{2}\right) \oplus \mathcal{P}_{\phi_{1}, \phi_{2}}\left(\Omega_{1} \times \Omega_{2}\right)
$$

Tensor Native Space der Native Spaces $\mathcal{N}_{\phi_{1}, \mathcal{P}_{1}}\left(\Omega_{1}\right)$ und $\mathcal{N}_{\phi_{2}, \mathcal{P}_{2}}\left(\Omega_{2}\right)$. Im d-variaten Fall heißt

$$
\begin{aligned}
& \mathcal{N}_{\phi_{1}, \ldots, \phi_{d}, \mathcal{P}_{1}, \ldots, \mathcal{P}_{d}}^{\otimes}\left(\Omega_{1} \times \ldots \times \Omega_{d}\right) \\
& \quad:=\mathcal{F}_{\phi_{1}, \mathcal{P}_{1}}\left(\Omega_{1}\right) \otimes_{\beta} \ldots \otimes_{\beta} \mathcal{F}_{\phi_{d}, \mathcal{P}_{d}}\left(\Omega_{d}\right) \oplus \mathcal{P}_{\phi_{1}, \ldots, \phi_{d}}\left(\Omega_{1} \times \ldots \times \Omega_{d}\right)
\end{aligned}
$$

bei entsprechender Wahl des Nullraumes $\mathcal{P}_{\phi_{1}, \ldots, \phi_{d}}\left(\Omega_{1} \times \ldots \times \Omega_{d}\right)$ Tensor Native Space der Native Spaces $\mathcal{N}_{\phi_{1}, \mathcal{P}_{1}}\left(\Omega_{1}\right), \ldots, \mathcal{N}_{\phi_{d}}, \mathcal{P}_{d}\left(\Omega_{d}\right)$.

\subsection{Polynom-Interpolation}

Aufgrund von Lemma 4.1 sehen wir, daß wir für eine Funktion $f$ aus einem SmolyakRaum $\mathcal{S}_{q, d}^{\mathcal{F}_{1}, \ldots, \mathcal{F}_{q-d+1}}$ immer ein Smolyak-Polynom finden können, so daß es $f$ in den Punkten eines passenden verschachtelten dünnen Gitters interpoliert. Es gilt sogar: 
Lemma 4.28 Die Interpolation im Smolyak-Polynomraum $\mathcal{S P}_{q, d}^{m_{1}, \ldots, m_{q-d+1}}$ mit $q, d \in \mathbb{N}, q \geq d$ und $m_{j} \in \mathbb{N}$ falls $1 \leq j \leq q-d+1$ ist auf den Datenpunkten des dünnen Gitters

$$
H_{q, d}^{m_{1}, \ldots, m_{q-d+1}}=\bigcup_{|i|_{d}=q}\left(X_{i_{1}} \otimes \cdots \otimes X_{i_{d}}\right)
$$

mit den verschachtelten Datensätzen $X_{j}$ mit $\left|X_{j}\right|=m_{j}$ für $1 \leq j \leq q-d+1$ stets eindeutig möglich.

Beweis: Da das dünne Gitter aus dem kartesischen Produkt von verschachtelten Datensätzen besteht, können wir auch $H_{q, d}^{m_{1}, \ldots, m_{q-d+1}}=\bigcup_{|i|_{d} \leq q}\left(X_{i_{1}} \times \ldots \times X_{i_{d}}\right)$ schreiben; damit wird offensichtlich, daß die Dimension des Smolyak-Polynomraumes $\mathcal{S P}_{q, d}^{m_{1}, \ldots, m_{q-d+1}}$ der Anzahl der Punkte des dünnen Gitters $H_{q, d}^{m_{1}, \ldots, m_{q-d+1}}$ entspricht. Der Rest folgt mit Lemma 4.1.

Wir betrachten im folgenden Smolyak-Polynominterpolanten der Gestalt $A_{q, d} f \in \mathcal{S P}_{q, d}^{m_{1}, \ldots, m_{q-d+1}}$ für alle $f \in \mathcal{F} \otimes \ldots \otimes \mathcal{F}$, wobei die grundlegenden univariaten Operatoren $U_{j}, 1 \leq j \leq q-d+1$, auf den Banachraum $\mathcal{F}$ wirken.

Eine Fehlerabschätzung für ein spezielles verschachteltes dünnes Gitter finden wir in [5]. Barthelmann, Novak und Ritter wählen dort als Gitterpunkte die Extremwerte der Tschebyscheffpolynome und erhalten mit Hilfe der für diese Wahl der Punkte bekannten Lebesgueschen Konstanten eine Fehlerschranke für eine solche Polynominterpolation. Das folgende Ergebnis folgt im wesentlichen [5]:

Satz 4.29 Sei $\mathcal{V}=\mathcal{C}_{c}^{\nu}$ der Raum der stetigen Funktionen mit kompaktem Träger. Im Tensorproduktraum $\mathcal{F} \otimes \ldots \otimes \mathcal{F}$ mit dem Banachraum $\mathcal{F} \subseteq C^{\nu}([a, b]), \nu \in N_{0}$, dessen Norm äquivalent zur $\|\cdot\|_{\infty}$-Norm ist, und zu $\mathcal{V} \otimes \ldots \otimes \mathcal{V}$ gleichmäßig verträglicher Norm gilt für $m_{j}=2^{j-1}+1$ und $1 \leq j \leq q-d+1$ die Ungleichung

$$
\left\|I_{d}-A_{q, d}\right\|_{\mathcal{F} \otimes \ldots \otimes \mathcal{F}, \mathcal{V} \otimes \ldots \otimes \mathcal{V}} \leq c d \max \left(2^{\nu}, 2 c\right)^{\frac{d-1}{2}} \cdot 2^{-\nu q} q^{2 d-1} .
$$

Im Clenshaw-Curtis-Fall folgt

$$
\left\|I_{d}-A_{q, d}\right\|_{\mathcal{F} \otimes \ldots \otimes \mathcal{F}, \mathcal{V} \otimes \ldots \otimes \mathcal{V}} \leq c 2^{\nu d} d \max (1,2 c)^{\frac{d-1}{2}} \cdot 2^{-\nu q} q^{2 d-1} .
$$

Dabei benutzt der auf den univariaten Polynominterpolationsoperatoren $U_{j}: \mathcal{F} \rightarrow \mathbb{P}_{m_{j}}$ aufbauende Smolyak-Operator $A_{q, d}$ ein spezielles dünnes Gitter

$$
H_{q, d}=\bigcup_{q-d+1 \leq|i|_{d} \leq q}\left(X_{i_{1}} \times \ldots \times X_{i_{d}}\right)
$$


wobei

$$
X_{j}=\left\{x_{j k} \quad: \quad x_{j k}=-\cos \left(\frac{\pi(k-1)}{m_{j}-1}\right), 1 \leq k \leq m_{j}\right\}
$$

gilt.

Der Beweis für $m_{j}=2^{j-1}+1,1 \leq j \leq q-d+1$, kann bei [5] nachgelesen werden. Die Abschätzung für den Clenshaw-Curtis-Fall erhalten wir, wenn wir die Konstante der univariaten Fehlerabschätzungen in [5] mit $2^{\nu}$ multiplizieren und dann das Resultat entsprechend korrigieren.

Korollar 4.30 Es gelten die Voraussetzungen von Satz 4.29. Dann folgern wir in Abhängigkeit von der Anzahl der Punkte des dünnen Gitters $n:=n(q, d)$ die Fehlerabschätzung

$$
\left\|I_{d}-A_{q, d}\right\|_{\mathcal{F} \otimes \ldots \otimes \mathcal{F}, \mathcal{V} \otimes \ldots \otimes \mathcal{V}} \leq C_{d, \nu} n^{-\nu}(2 d+\log (n))^{(\nu+2)(d-1)+1}
$$

mit

$$
C_{d, \nu}=\frac{c d 2^{\nu}}{(d-1) ! \nu} \max \left(2 c, c_{\nu}\right)^{d-1}
$$

und $c_{\nu}=1$ im Clenshaw-Curtis-Fall und $c_{\nu}=2^{\nu}$ im Fall $m_{j}=2^{j-1}+1$.

\subsection{Interpolation mit Splines}

Wir betrachten in diesem Abschnitt zunächst univariate Interpolanten aus dem linearen Raum der polynomialen Splines

$$
\mathcal{S}_{k}(X):=\left\{s \in C^{k-1}(\Omega):\left.s\right|_{\left[x_{i-1}, x_{i}\right]} \in \mathbb{P}_{k-1}^{1}, 1 \leq i \leq N+1\right\},
$$

wobei $X=\left\{x_{1}, \ldots, x_{N+1}\right\} \subseteq \Omega$ eine Knotenfolge mit

$$
a=x_{1}<\ldots<x_{N+1}=b
$$

und $\Omega=[a, b]$ bezeichnet. Stellt

$$
U_{j, X_{j}} f=s_{j}
$$

eine Spline-Interpolante an eine Funktion $f \in \mathcal{F}_{j} \subseteq \mathcal{F}$ auf der Knotenfolge $X_{j}$ dar, so ist $U_{j, X_{j}}$ ein linearer Operator, der den $\operatorname{Raum} \mathcal{F}_{j}$ auf einen $\operatorname{Raum} \mathcal{V}_{j} \subseteq \mathcal{V}$ mit 
$\mathcal{S}_{k_{j}}\left(X_{j}\right), \mathcal{F}_{j} \subseteq \mathcal{V}_{j}$ abbildet. Gilt zusätzlich $X_{1} \subseteq \ldots \subseteq X_{q-d+1}$ für $q \geq d$, so können wir nach Lemma $4.1 \mathrm{zu}$ einem $d$-variaten $f \in \mathcal{S}_{q, d}^{\mathcal{F}_{1}, \ldots, \mathcal{F}_{q-d+1}}$ eine Smolyak-SplineInterpolante der Gestalt $A_{q, d} f$ mit zugehörigen univariaten Interpolationsoperatoren $U_{j}:=U_{j, X_{j}}$ auf einer $d$-dimensionalen Knotenfolge

$$
H_{q, d}=\bigcup_{|j|_{d}=q}\left(X_{j_{1}} \times \ldots \times X_{j_{d}}\right)
$$

in Form eines dünnen Gitters mit $m_{j}=N_{j}+1$ für alle $j$ finden. Ist die Norm des Raumes $\mathcal{F} \otimes \ldots \otimes \mathcal{F}$ mit der Norm von $\mathcal{V} \otimes \ldots \otimes \mathcal{V}$ gleichmäßig verträglich, so kann der Fehler der multivariaten Interpolation unter Verwendung des univariaten mit Hilfe von Satz 4.2 bestimmt werden. Im folgenden werden wir nun solche Fehlerabschätzungen für einige konkrete Fälle bereitstellen. Dabei bezeichne $h_{j}:=\max _{1 \leq i \leq N_{j}}\left|x_{j i+1}-x_{j i}\right|$ den Abstand der Knotenpunkte der $j$-ten Knotenfolge; die betrachteten Knotenpunkte seien $X_{j}=\left\{x_{j 1}, \ldots, x_{j N_{j}+1}\right\}$ für $1 \leq j \leq q-d+1$. Wir starten mit dem einfachsten Fall.

Lemma 4.31 Zu jedem $f \in C^{2}\left(\Omega_{j}\right)$ sei $U_{j, X_{j}} f:=s_{j} \in \mathcal{S}_{1}\left(X_{j}\right)$ der eindeutig bestimmte Polygonzug mit $s_{j}\left(x_{j l_{j}}\right)=f\left(x_{j l_{j}}\right)$ für $1 \leq j \leq q-d+1$ und $1 \leq l_{j} \leq N_{j}+1$. Sei $A_{q, d}$ der zugehörige Smolyak-Spline-Interpolationsoperator. Dann gilt mit $\Omega:=\Omega_{1} \times \ldots \times \Omega_{d}$

$$
\left\|I_{d}-A_{q, d}\right\|_{\mathcal{F}_{d}^{r}(\Omega), C(\Omega)} \leq \frac{h^{2} \beta^{-2(q-d+1)}}{8}\left(\begin{array}{c}
q \\
d-1
\end{array}\right) \begin{cases}1 & : h \leq \frac{2 \sqrt{2}}{\sqrt{1+\beta^{-2}}} \\
\left(\frac{\left(1+\beta^{-2}\right) h^{2}}{8}\right)^{d-1} & : \text { sonst }\end{cases}
$$

für alle $r \geq 2$ und $h_{j}=h \beta^{-j}$.

Beweis: Wegen

$$
\left|f(x)-s_{j}(x)\right| \leq \frac{1}{8}\left\|f^{\prime \prime}\right\|_{\infty} h_{j}^{2}
$$

vgl. [35], folgern wir

$$
\left\|I_{1}-U_{j}\right\|_{\mathcal{F}_{1}^{r}\left(\Omega_{j}\right), C\left(\Omega_{j}\right)} \leq \frac{h_{j}^{2}}{8}
$$

und nutzen dann Korollar 4.3 aufgrund der gleichmäßigen Verträglichkeit von $\|\cdot\|_{\mathcal{F}_{d}^{r}(\Omega)}$ mit $\|\cdot\|_{C(\Omega)}$.

Damit folgt: 
Korollar 4.32 Unter den Voraussetzungen von Lemma 4.31 gilt für $\beta \geq 1$

$$
\left\|I_{d}-A_{q, d}\right\|_{\mathcal{F}_{d}^{r}(\Omega), C(\Omega)} \leq n^{-2 \log (\beta)}(2 d+\log (n))^{(d-1)(2 \log (\beta)+1)} K_{d}(\beta, h)
$$

mit

$$
K_{d}(\beta, h):=\frac{h^{2} \beta^{-2 \log ((d-1) !)}}{8(d-1) !}\left\{\begin{array}{ll}
1 & : \quad h \leq \frac{2 \sqrt{2}}{\sqrt{1+\beta^{-2}}} \\
\left(\frac{\left(1+\beta^{-2}\right) h^{2}}{8}\right)^{d-1} & : \text { sonst }
\end{array} .\right.
$$

Dabei bezeichnet $n:=n(q, d)$ die Anzahl der Punkte des dünnen Gitters $H_{q, d}^{C C}$.

Für kubische Splines gilt folgende Fehlerabschätzung:

Lemma 4.33 Für $f \in C^{4}\left(\Omega_{j}\right)$ mit $\Omega_{j}=\left[a_{j}, b_{j}\right]$ sei $U_{j, X_{j}} f$ der eindeutig bestimmte Spline $s_{j} \in \mathcal{S}_{3}\left(X_{j}\right)$, wobei $s_{j}\left(x_{j l_{j}}\right)=f\left(x_{j l_{j}}\right)$ für $1 \leq j \leq q-d+1$ und $1 \leq l_{j} \leq N_{j}+1$ gelte. Zudem sei entweder

$$
\begin{array}{rlr}
s_{j}^{\prime}\left(a_{j}\right)-f^{\prime}\left(a_{j}\right) & =s_{j}^{\prime}\left(b_{j}\right)-f^{\prime}\left(b_{j}\right)=0 & \text { oder } \\
s_{j}^{\prime \prime}\left(a_{j}\right)-f^{\prime \prime}\left(a_{j}\right) & =s_{j}^{\prime \prime}\left(b_{j}\right)-f^{\prime \prime}\left(b_{j}\right)=0 &
\end{array}
$$

erfüllt. Dann gilt für den zugehörigen Smolyak-Spline-Interpolationsoperator $A_{q, d}$ mit $r \geq 4, h_{j}=h \beta^{-j}$ und $\Omega:=\Omega_{1} \times \ldots \times \Omega_{d}$ die Ungleichung

$$
\left\|I_{d}-A_{q, d}\right\|_{\mathcal{F}_{d}^{r}(\Omega), C(\Omega)} \leq \frac{5 h^{4} \beta^{-4(q-d+1)}}{384}\left(\begin{array}{c}
q \\
d-1
\end{array}\right) \begin{cases}1 & : h \leq \frac{3 \sqrt{2}}{\left(5+5 \beta^{-4}\right)^{\frac{1}{4}}} \\
\left(\frac{5\left(1+\beta^{-4}\right) h^{4}}{384}\right)^{d-1} & : \text { sonst }\end{cases}
$$

Beweis: Nach [14] gilt

$$
\left\|f-s_{j}\right\|_{\infty} \leq \frac{5}{384} h_{j}^{4}\left\|f^{(4)}\right\|_{\infty}
$$

Daraus folgern wir

$$
\left\|I_{1}-U_{j}\right\|_{\mathcal{F}_{1}^{r}(\Omega), C(\Omega)} \leq \frac{5}{384} h_{j}^{4},
$$

und es ergibt sich mit Korollar 4.3

$$
\left\|I_{d}-A_{q, d}\right\|_{\mathcal{F}_{d}^{r}(\Omega), C(\Omega)} \leq \frac{5 h^{4}}{384} \beta^{-4(q-d+1)}\left(\begin{array}{c}
q \\
d-1
\end{array}\right) \max \left(1,\left(1+\beta^{-4}\right) \frac{5 h^{4}}{384}\right)^{d-1} .
$$

Die Schranke erhält in Abhängigkeit von der Punktanzahl des dünnen Gitters $H_{q, d}^{C C}$ die folgende Gestalt. 
Korollar 4.34 Es gelten die Voraussetzungen von Lemma 4.33. Dann folgt mit $\beta \geq 1$

$$
\left\|I_{d}-A_{q, d}\right\|_{\mathcal{F}_{d}^{r}(\Omega), C(\Omega)} \leq n^{-4 \log (\beta)}(2 d+\log (n))^{(d-1)(4 \log (\beta)+1)} K_{d}(\beta, h),
$$

wobei

$$
K_{d}(\beta, h):=\frac{5 h^{4} \beta^{-4 \log ((d-1) !)}}{384(d-1) !}\left\{\begin{array}{ll}
1 & : \quad h \leq \frac{3 \sqrt{2}}{\left(5+5 \beta^{-4}\right)^{\frac{1}{4}}}
\end{array},\right.
$$

und $n:=n(q, d)$ die Anzahl der Punkte des Clenshaw-Curtis-Gitters bezeichnet.

Eine allgemeinere, aber weniger konkrete Schranke ergibt sich aus

Lemma 4.35 Zu $f \in \mathcal{F}_{1, L_{2}}^{2 m}\left(\Omega_{j}\right)$ gelten für das eindeutig bestimmte Element $U_{j, X_{j}} f=s_{j} \in \mathcal{S}_{2 m}\left(X_{j}\right)$ die Gleichungen $f\left(x_{j l_{j}}\right)=s_{j}\left(x_{j l_{j}}\right)$ und

$$
f^{(k)}\left(a_{j}\right)-s_{j}^{(k)}\left(a_{j}\right)=f^{(k)}\left(b_{j}\right)-s_{j}^{(k)}\left(b_{j}\right)=0
$$

mit $\Omega_{j}=\left[a_{j}, b_{j}\right], 0 \leq k \leq m-1,1 \leq j \leq q-d+1$ und $1 \leq l_{j} \leq N_{j}+1$. Dann folgt mit $h_{j}=h \cdot \beta^{-j}$ für den entsprechenden Smolyak-Spline-Interpolationsoperator $A_{q, d}$

$$
\left\|I_{d}-A_{q, d}\right\|_{\mathcal{F}_{d, L_{2}}^{2 m}(\Omega), L_{2}(\Omega)} \leq K h^{2 m} \beta^{-2 m(q-d+1)}\left(\begin{array}{c}
q \\
d-1
\end{array}\right) \max \left(1,\left(1+\beta^{-2 m}\right) K h^{2 m}\right) .
$$

Beweis: Nach [44] gilt für $0 \leq k \leq 2 m-1$

$$
\|f-s\|_{L_{2}\left(\Omega_{j}\right)} \leq K h_{j}^{2 m}\|f\|_{\mathcal{F}_{1, L_{2}}^{2 m}\left(\Omega_{j}\right)} .
$$

Wir schlußfolgern daraus

$$
\left\|I_{1}-U_{j}\right\|_{\mathcal{F}_{1, L_{2}}^{2 m}\left(\Omega_{j}\right), L_{2}\left(\Omega_{j}\right)} \leq K h_{j}^{2 m}
$$

und damit ergibt sich aufgrund der gleichmäßigen Verträglichkeit von $\|\cdot\|_{\mathcal{F}_{d, L_{2}}^{2 m}(\Omega)}$ und $\|\cdot\|_{L_{2}(\Omega)}$ mit Korollar 4.3 die Behauptung.

Es folgt: 
Korollar 4.36 Unter den Voraussetzungen von Lemma 4.35 gilt mit $n:=n(q, d)=\left|H_{q, d}^{C C}\right|$

$$
\left\|I_{d}-A_{q, d}\right\|_{\mathcal{F}_{d, L_{2}}^{2 m}(\Omega), L_{2}(\Omega)} \leq n^{-2 m \log (\beta)}(2 d+\log (n))^{(d-1)(2 m \log (\beta)+1)} K_{d, m}(\beta, h) .
$$

Dabei sei

$$
K_{d, m}(\beta, h):=\frac{K h^{2 m}}{(d-1) !} \beta^{-2 m \log ((d-1) !)} \max \left(1,\left(1+\beta^{-2 m}\right) K h^{2 m}\right)^{d-1} .
$$

In den folgenden zwei Lemmata benutzen wir explizit äquidistante Knotenfolgen.

Lemma 4.37 Es gelte $f \in \mathcal{F}_{1, L_{p}}^{r+1}\left(\Omega_{j}\right), \Omega_{j}=\left[a_{j}, b_{j}\right]$; sei $U_{j, X_{j}} f=s_{j} \in \mathcal{S}_{2 m}\left(X_{j}\right)$ der eindeutig bestimmte Spline mit $f\left(x_{j l_{j}}\right)=s_{j}\left(x_{j l_{j}}\right)$ für $1 \leq l_{j} \leq N_{j}$ und $1 \leq j \leq q-d+1$,

$$
\begin{aligned}
& f^{(2 k-1)}\left(a_{j}\right)-s_{j}^{(2 k-1)}\left(a_{j}\right)=f^{(2 k-1)}\left(b_{j}\right)-s_{j}^{(2 k-1)}\left(b_{j}\right)=0 \quad \text { für } \quad 1 \leq 2 k-1 \leq r, \\
& s_{j}^{(2 k-1)}\left(a_{j}\right)=s_{j}^{(2 k-1)}\left(b_{j}\right)=0 \quad \text { für } \quad r<2 k-1 \leq 2 m-3
\end{aligned}
$$

und äquidistanter Knotenfolge $X_{j}$. Dann gilt mit $h_{j}=h \beta^{-j}$

$$
\begin{aligned}
& \left\|I_{d}-A_{q, d}\right\|_{\mathcal{F}_{d, L_{p}}^{\mu}(\Omega), L_{p}(\Omega)} \\
& \leq K h^{r+1} \beta^{-(r+1)(q-d+1)}\left(\begin{array}{c}
q \\
d-1
\end{array}\right) \max \left(1,\left(1+\beta^{-(r+1)}\right) K h^{r+1}\right)^{d-1}
\end{aligned}
$$

für alle $\mu \geq r+1$.

Beweis: Wir wenden die Ergebnisse von [44] an. Danach gilt

$$
\left\|I_{1}-U_{j}\right\|_{\mathcal{F}_{1, L_{p}}^{\mu}(\Omega), L_{p}(\Omega)} \leq K h_{j}^{r+1}
$$

und wir folgern mit Korollar 4.3 die Behauptung.

Lemma 4.38 Für $0 \leq r<2 m$ sei $f \in \mathcal{F}_{d, L_{p}}^{r+1}(\Omega)$. Sei $U_{j, X_{j}} f=s_{j} \in \mathcal{S}_{2 m}(X)$ der eindeutig bestimmte Spline mit $f\left(x_{j l_{j}}\right)=s_{j}\left(x_{j l_{j}}\right)$ für $1 \leq l_{j} \leq N_{j}+1,1 \leq j \leq q-d+1$,

$$
\begin{array}{rlll}
f^{(k)}\left(a_{j}\right)-s_{j}^{(k)}\left(a_{j}\right)= & f^{(k)}\left(b_{j}\right)-s_{j}^{(k)}\left(b_{j}\right)=0 & \text { für } \quad 0 \leq k \leq \min (r, m-1), \\
s_{j}^{(k)}\left(a_{j}\right)=s_{j}^{(k)}\left(b_{j}\right)=0 & \text { für } \quad \min (r, m-1)<k \leq m-1
\end{array}
$$


mit äquidistanter Knotenfolge $X_{j}$. Dann gilt für den Smolyak-Spline-Interpolationsoperator $A_{q, d}$

$$
\begin{aligned}
& \left\|I_{d}-A_{q, d}\right\|_{\mathcal{F}_{d, L_{p}}^{\mu}(\Omega), L_{p}(\Omega)} \\
& \quad \leq K h^{r+1} \beta^{-(r+1)(q-d+1)}\left(\begin{array}{c}
q \\
d-1
\end{array}\right) \max \left(1,\left(1+\beta^{-(r+1)}\right) K h^{r+1}\right)^{d-1}
\end{aligned}
$$

für alle $\mu \geq r+1$ und $h_{j}=h \beta^{-j}$.

Beweis: Mit [44] folgern wir $\left\|I_{1}-U_{j}\right\|_{\mathcal{F}_{1, L_{p}}^{\mu}(\Omega), L_{p}(\Omega)} \leq K h_{j}^{r+1}$ und mit Korollar 4.3 die Behauptung.

In Abhängigkeit von den Gitterpunkten eines äquidistanten dünnen Gitters gilt:

Korollar 4.39 Unter den Voraussetzungen von Lemma 4.37 bzw. von Lemma 4.38 folgt mit $n:=n(q, d)=\left|H_{q, d}^{C C}\right|$

$$
\left\|I_{d}-A_{q, d}\right\|_{\mathcal{F}_{d, L_{p}}^{\mu}(\Omega), L_{p}(\Omega)} \leq n^{-(r+1) \log (\beta)}(2 d+\log (n))^{(d-1)((r+1) \log (\beta)+1)} K_{d, r}(\beta, h)
$$

und

$$
K_{d, r}(\beta, h):=\frac{K h^{r+1}}{(d-1) !} \beta^{-(r+1) \log ((d-1) !)} \max \left(1,\left(1+\beta^{-(r+1)}\right) K h^{r+1}\right)^{d-1} .
$$

\subsection{Interpolation mit Basisfunktionen}

In diesem Abschnitt wollen wir den Smolyak-Algorithmus auf die Interpolation mit Basisfunktionen auf dem dünnen Gitter anwenden. Um die vorangegangenen Ergebnisse benutzen zu können, benötigen wir eine univariate, positiv definite Basisfunktion $\phi$ und Datensätze $X_{1}, \ldots, X_{q-d+1}$ mit $X_{j}=\left\{x_{j 1}, \ldots, x_{j m_{j}}\right\}$ und $X_{j} \subseteq X_{j+1}$. Wir definieren mit $f \in \mathcal{N}_{\phi}\left(\Omega_{j}\right) \subset C\left(\Omega_{j}\right)$ und $a_{j k} \in \mathbb{R}$ für alle $j, k$

$$
U_{j} f=s_{f, \phi, X_{j}}:=\sum_{i_{j}=1}^{m_{j}} a_{j i_{j}} \phi\left(\cdot, x_{j i_{j}}\right) .
$$

Nach Satz 2.2 sind die Gleichungssysteme

$$
U_{j} f(x)=f(x) \text { für alle } x \in X_{j}
$$


eindeutig lösbar. Mit Lemma 4.1 folgern wir, daß

$$
A_{q, d} f(x)=I_{d} f(x)
$$

für alle $x \in H_{q, d}$ und für alle $f \in \mathcal{N}_{\phi}\left(\Omega_{1}\right) \otimes \ldots \otimes \mathcal{N}_{\phi}\left(\Omega_{d}\right)=\mathcal{N}_{\phi \otimes \ldots \otimes \phi}(\Omega)$ mit $\Omega:=\Omega_{1} \times \ldots \times \Omega_{d}$ gilt. Da die Norm des Native Space $\|\cdot\|_{\beta, \mathcal{N}_{\phi}\left(\Omega_{1}\right) \otimes \ldots \otimes \mathcal{N}_{\phi}\left(\Omega_{d}\right)}=\|\cdot\|_{\phi \otimes \ldots \otimes \phi}$ mit der Norm $\|\cdot\|_{\infty, \Omega}=\|\cdot\|_{\lambda, \mathcal{C}_{k}\left(\Omega_{1}\right) \otimes \ldots \otimes \mathcal{C}_{k}\left(\Omega_{d}\right)}$ gleichmäßig verträglich ist, läßt sich der Interpolationsfehler mit Satz 4.2 abschätzen.

Wir benötigen dazu aber zunächst noch folgenden

Satz 4.40 Sei $I_{1}: \mathcal{N}_{\phi}(\Omega) \rightarrow \mathcal{V}$ für $\mathcal{V}=C(\Omega)$ der Einbettungsoperator; im Fall $\mathcal{V}=\mathbb{R}$ sei $I_{1}$ der Punktauswertungsoperator. Es gelte $\|\phi\|_{\infty}<\infty$. Dann folgt

$$
\left\|I_{1}\right\|_{\phi, \mathcal{V}} \leq c^{*}\|\phi\|_{\infty}
$$

mit $c^{*} \in \mathbb{R}_{+}$.

Beweis: Wir wählen zunächst

$$
f=\sum_{j=1}^{N} \lambda_{j} \phi\left(\cdot, x_{j}\right) \quad \in F_{\phi}(\Omega) .
$$

Es folgt mit $\lambda=\left(\lambda_{1}, \ldots, \lambda_{N}\right)$

$$
\|f\|_{\infty} \leq \sum_{j=1}^{N}\left|\lambda_{j}\right|\|\phi\|_{\infty}=|\lambda|\|\phi\|_{\infty} \leq c\left(\lambda^{T} \lambda\right)^{\frac{1}{2}}\|\phi\|_{\infty} ;
$$

aufgrund der positiven Definitheit von $\phi$

$$
\|f\|_{\phi}^{2}=\sum_{j, k=1}^{N} \lambda_{j} \lambda_{k} \phi\left(x_{j}, x_{k}\right) \geq \tilde{c} \lambda^{T} \lambda
$$

und damit

$$
\|f\|_{\infty} \leq C\|\phi\|_{\infty}\|f\|_{\phi} .
$$

Sei nun $f_{n} \in F_{\phi}(\Omega)$ eine Cauchyfolge mit Grenzwert $f$. Es existiert dann zu jedem $\epsilon>0, \epsilon \in \mathbb{R}$ ein $M(\epsilon) \in \mathbb{N}$ mit

$$
\left\|f_{n}-f\right\|_{\phi} \leq \epsilon .
$$


Es gilt

$$
\begin{aligned}
f(x) & =(f, \phi(\cdot, x))_{\phi} \\
& =\left(f-f_{n}, \phi(\cdot, x)\right)_{\phi}+\left(f_{n}, \phi(\cdot, x)\right)_{\phi} \\
& \leq\left\|f-f_{n}\right\|_{\phi}\|\phi(\cdot, x)\|_{\phi}+f_{n}(x) \\
& <\epsilon(\phi(\cdot, x), \phi(\cdot, x))_{\phi}^{\frac{1}{2}}+f_{n}(x) \\
& =\epsilon \phi(x, x)^{\frac{1}{2}}+f_{n}(x)
\end{aligned}
$$

und daraus folgern wir

$$
\begin{aligned}
\|f\|_{\infty} & <\epsilon\|\phi\|_{\infty}^{\frac{1}{2}}+\left\|f_{n}\right\|_{\infty} \\
& \leq \epsilon\|\phi\|_{\infty}^{\frac{1}{2}}+C\|\phi\|_{\infty}\|f\|_{\phi} \\
& \leq(\tilde{\epsilon}+C)\|\phi\|_{\infty}\|f\|_{\phi},
\end{aligned}
$$

und es folgt die Behauptung für den Einbettungsoperator; offenbar verläuft der Beweis für den Punktauswertungsoperator analog.

Wir werden im folgenden nun eine Fehlerabschätzung für eine Smolyak-Interpolation mit Wendlandfunktionen angeben.

Satz 4.41 Seien $\phi:=\phi_{k+1, k}$ univariate Wendlandfunktionen; wir bezeichnen die zugehörigen Basisfunktionen-Interpolanten an $f \in \mathcal{N}_{\phi}\left(\Omega_{j}\right)$ mit $s_{f, \phi, X_{j}}=U_{j} f$. Unter den Voraussetzungen von Satz 2.9 folgt dann für den zugehörigen Smolyak-Interpolationsoperator $A_{q, d}$

$$
\begin{aligned}
& \left\|I_{d}-A_{q, d}\right\|_{\Phi, \infty} \\
& \quad \leq c h^{k+\frac{1}{2}} \beta^{-(q-d+1)\left(k+\frac{1}{2}\right)}\left(\begin{array}{c}
q \\
d-1
\end{array}\right) \max \left(c^{*} C_{k}, c h^{k+\frac{1}{2}}\left(1+\beta^{-\left(k+\frac{1}{2}\right)}\right)\right)^{d-1}
\end{aligned}
$$

mit der d-variaten Basisfunktion $\Phi:=\phi \otimes \ldots \otimes \phi$, den Fülldistanzen $h_{j}:=h \beta^{-j}$, $\beta \geq 1$ und $C_{k}=\left\{\begin{array}{lll}1 & : & k=0 \\ \frac{B(2 k, k+1)}{2^{k-1} \Gamma(k)} & : & \text { sonst }\end{array}\right.$

Beweis: Wir folgern das Ergebnis mit Korollar 4.3 aufgrund von

$$
\left\|I_{1}-U_{j}\right\| \leq c h_{j}^{k+\frac{1}{2}}
$$


aus [47]; weiterhin schätzen wir die Norm der Wendlandfunktion

$$
\|\phi\|_{\infty} \leq C_{k}
$$

ab, da die Funktion nach [37] im Ursprung ihr Maximum $C_{k}$ annimmt.

Korollar 4.42 Unter den Voraussetzungen von Satz 4.41 gilt mit der Punktanzahl $n:=n(q, d)$ des Clenshaw-Curtis-Gitters

$$
\left\|I_{d}-A_{q, d}\right\|_{\Phi, \infty} \leq n^{-\log (\beta)\left(k+\frac{1}{2}\right)}(2 d+\log (n))^{(d-1)\left(\left(k+\frac{1}{2}\right) \log (\beta)+1\right)} K_{d, k}(\beta, h)
$$

mit

$$
K_{d, k}(\beta, h):=\frac{c h^{k+\frac{1}{2}}}{(d-1) !} \beta^{-\left(k+\frac{1}{2}\right) \log ((d-1) !)} \max \left(c^{*} C_{k}, c h^{k+\frac{1}{2}}\left(1+\beta^{-\left(k+\frac{1}{2}\right)}\right)\right)^{d-1} .
$$

Im Fall $h_{j}=2^{-j}$ folgern wir

$$
\left\|I_{d}-A_{q, d}\right\|_{\Phi, \infty} \leq n^{-\left(k+\frac{1}{2}\right)}(2 d+\log (n))^{(d-1)\left(k+\frac{3}{2}\right)} K_{d, k}
$$

mit

$$
K_{d, k}:=\frac{c}{(d-1) !^{k+\frac{3}{2}}} \max \left(c^{*} C_{k}, c\left(1+2^{-\left(k+\frac{1}{2}\right)}\right)\right)^{d-1} .
$$

Im Rahmen der Interpolation mit radialen Wendlandfunktionen $\Phi$ erhalten wir bei Nutzung eines quasi-regulären Datensatzes $X$ mit $|X|=N$ auf dem beschränkten Gebiet eine Fehlerabschätzung vom Typ

$$
\left\|f-s_{f, \Phi, X}\right\|_{\infty} \leq c_{d, k} N^{-\left(\frac{k+\frac{1}{2}}{d}\right)} .
$$

Dabei bezeichnet $s_{f, \Phi, X}$ die Interpolante an $f$ auf dem Datensatz $X$. Offensichtlich wird unter Verwendung einer Smolyak-Interpolante der Gestalt

$$
\sum_{\substack{q-d+1 \leq i \mid \leq q \\
i \in \mathbb{N}^{d}}}(-1)^{q-|i|}\left(\begin{array}{c}
q-|i| \\
d-1
\end{array}\right)\left(s_{f, \phi, X_{i_{1}}} \otimes \ldots \otimes s_{f, \phi, X_{i_{d}}}\right)
$$

der Fluch der Dimension beseitigt.

Um weitere Beispiele zu finden, formulieren wir zunächst die folgende 
Definition 4.43 Ein abgeschlossenes kompaktes Gebiet $\Omega \subseteq \mathbb{R}^{d}$ mit nichtleerem Inneren genügt einer inneren Kegelbedingung, falls es einen festen Winkel $\gamma>0$ und eine feste Höhe $\delta$ gibt, so daß jeder beschränkte Punkt $x$ Scheitelpunkt eines in $\Omega$ liegenden zylindrischen Kegels mit Winkel $\gamma$ und Höhe $\delta$ ist.

Wir wenden uns nun den Gaußglocken zu. Hier wählen wir $\mathcal{V}=\mathbb{R}$ und damit $\mathcal{V} \otimes \ldots \otimes \mathcal{V}=\mathbb{R}$, da die Gaußglocke keinen kompakten Träger besitzt und wir Satz 4.5 anstelle von Satz 4.8 anwenden.

Satz 4.44 Sei $U_{j}: \mathcal{N}_{\phi}\left(\Omega_{j}\right) \rightarrow C\left(\Omega_{j}\right)$ der zur Basisfunktion $\phi$ mit $\phi(x):=\phi_{\alpha}(x)=$ $e^{-\alpha|x|^{2}}, x \in \Omega_{j}$, gehörige Interpolationsoperator, wobei die Gebiete $\Omega_{j}$ einer inneren Kegelbedingung genügen. Dann erfüllt der entsprechende Smolyak-Interpolationsoperator $A_{q, d}$ unter den Voraussetzungen von Satz 2.9 für $\Phi:=\phi \otimes \ldots \otimes \phi$ mit $h_{j}=h 2^{-j}, h \leq 1, q-d \rightarrow \infty$ und $x \in \Omega_{1} \times \ldots \times \Omega_{d}$ die Fehlerabschätzung

$$
\left|f(x)-A_{q, d} f(x)\right| \leq C e^{-2^{(q-d+1)} \frac{c}{h}}\left(\begin{array}{c}
q \\
d-1
\end{array}\right) \max \left(c^{*}, 2 c_{\phi}\right)^{d-1}\|f\|_{\Phi} .
$$

Beweis: Zunächst nutzen wir aus, daß nach [33] die Fehlerabschätzung

$$
\left\|I_{1}-U_{j}\right\|_{\phi, I R} \leq c_{\phi} h_{j}^{l}
$$

für alle $l \in I N_{0}$ gilt. Dabei erfüllt die Konstante $c_{\phi}$ für alle $h_{j}, h_{j}^{-1} \rightarrow \infty$ die Bedingung

$$
c_{\phi} h_{j}^{l} \leq C e^{-\frac{c}{h_{j}}}
$$

Aus Korollar 4.3 folgern wir

$$
\begin{aligned}
\left\|I_{d}-A_{q, d}\right\|_{\Phi, \mathbb{R}} & \leq c_{\phi} h^{l} 2^{-l(q-d+1)}\left(\begin{array}{c}
q \\
d-1
\end{array}\right) \max \left(c^{*}, c_{\phi} h^{l}\left(1+2^{-l}\right)\right)^{d-1} \\
& \leq c_{\phi} h_{q-d+1}^{l}\left(\begin{array}{c}
q \\
d-1
\end{array}\right) \max \left(c^{*}, c_{\phi} h^{l}\left(1+2^{-l}\right)\right)^{d-1} \\
& \leq C e^{-c h_{q-d+1}^{-1}}\left(\begin{array}{c}
q \\
d-1
\end{array}\right) \max \left(c^{*}, c_{\phi} h^{l}\left(1+2^{-l}\right)\right)^{d-1} \\
& \leq C e^{-2^{(q-d+1)} c h^{-1}}\left(\begin{array}{c}
q \\
d-1
\end{array}\right) \max \left(c^{*}, 2 c_{\phi}\right)^{d-1} .
\end{aligned}
$$


Korollar 4.45 Unter den Voraussetzungen von Satz 4.44 gilt für $n \rightarrow \infty$ für alle $x \in \mathbb{R}^{d}$

$$
\left|f(x)-A_{q, d} f(x)\right| \leq e^{-\frac{c}{h}(d-1) ! n(2 d+\log (n))^{-(d-1)}}(2 d+\log (n))^{d-1} K_{d, \phi}\|f\|_{\Phi}
$$

mit

$$
K_{d, \phi}=\frac{C}{(d-1) !} \max \left(c^{*}, 2 c_{\phi}\right)^{d-1}
$$

Dabei bezeichnet $n:=n(q, d)$ die Gitterpunktanzahl des dünnen Gitters $H_{q, d}^{C C}$.

Beweis: Wir benutzen die Abschätzung

$$
e^{-2^{(q-d+1)} \frac{c}{h}} \leq e^{-\frac{c}{h}(d-1) ! n(2 d+\log (n))^{-(d-1)}}
$$

und

$$
\left(\begin{array}{c}
q \\
d-1
\end{array}\right) \leq \frac{1}{(d-1) !}(2 d+\log (n))^{d-1}
$$

Bei der klassischen Interpolation mit Gaußglocken bekommen wir Fehlerschranken der Form

$$
\left\|f-s_{f, \Phi, X}\right\|_{\infty} \leq c_{d} e^{-c N^{\frac{1}{d}}}
$$

wobei $N=|X|$ die Anzahl der Daten eines quasi-regulären Datensatzes darstellt. Das Ergebnis von Korollar 4.45 zeigt, daß wir durch die Verwendung des SmolyakAlgorithmus bei der Interpolation mit Gaußglocken den Fluch der Dimension deutlich besänftigt haben.

Ein ähnliches Ergebnis erreichen wir für Inverse Multiquadrics:

Satz 4.46 Es sei $\phi(x):=\phi_{c, \beta}(x):=\left(c^{2}+x^{2}\right)^{-\frac{\beta}{2}}$ für $c \in \mathbb{R}_{+}, \beta \in \mathbb{R}_{+} \backslash 2 \mathbb{N}$ die Basisfunktion zur Interpolante $s_{f, \phi, X_{j}}=U_{j}(f)$ mit $f \in \mathcal{N}_{\phi}\left(\Omega_{j}\right)$ auf dem Datensatz $X_{j}$. Die Gebiete $\Omega_{j}$ genügen einer inneren Kegelbedingung; es seien die Voraussetzungen von Satz 2.9 erfüllt. Dann gilt mit $q-d \rightarrow \infty$ für den zugehörigen SmolyakInterpolationsoperator und für alle $x \in \Omega_{1} \times \ldots \times \Omega_{d}$

$$
\left|f(x)-A_{q, d} f(x)\right| \leq C e^{-2^{q-d+1} \frac{c}{h}}\left(\begin{array}{c}
q \\
d-1
\end{array}\right) \max \left(c^{-\beta}, 2 c_{\phi}\right)^{d-1}\|f\|_{\Phi} .
$$

mit $\Phi:=\phi \otimes \ldots \otimes \phi$. 
Der Beweis verläuft analog zu dem von Satz 4.44. Wir folgern:

Korollar 4.47 Es gelten die Voraussetzungen von Satz 4.46. Dann folgt

$$
\left|f(x)-A_{q, d} f(x)\right| \leq e^{-\frac{c}{h}(d-1) ! n(2 d+\log (n))^{-(d-1)}}(2 d+\log (n))^{d-1} K_{d, \phi}\|f\|_{\Phi}
$$

mit

$$
K_{d, \phi}:=\frac{C}{(d-1) !} \max \left(c^{-\beta}, 2 c_{\phi}\right)^{d-1} .
$$

Auch in diesem Fall haben wir durch Verwendung des Smolyak-Algorithmus den Fluch der Dimension entscheidend abgemildert, denn bei der klassischen Interpolation mit radialen Multiquadrics erzielen wir Fehlerabschätzungen der Form

$$
\left\|f-s_{f, \Phi, X}\right\|_{\infty} \leq c_{d} e^{-N^{\frac{1}{d}}}
$$

$X$ ist dabei wieder ein quasi-regulärer Datensatz mit $|X|=N$.

Wir fassen zusammen, daß die Methode von Smolyak auch bei der Interpolation mit radialen Basisfunktionen angewandt werden kann und dort Wirkung zeigt. Besonders hervorzuheben ist, daß der Fluch der Dimension nicht nur im Fall polynomialer, sondern auch bei exponentieller Konvergenz deutlich besänftigt werden kann. Allerdings ist bei Anwendung des Smolyak-Algorithmus nach der in diesem Abschnitt dargestellten klassischen Methode lediglich eine Nutzung von solchen Basisfunktionen, die sich als Tensorprodukt einer positiv definiten, univariaten Funktion schreiben lassen, möglich. Das wird sich im kommenden Abschnitt, in dem der Gebrauch allgemeiner Basisfunktionen möglich sein wird, ändern.

Ausblickend stellen wir weiterhin fest, daß eine ähnliche Vorgehensweise auch in allgemeineren Fällen, insbesondere bei bedingt positiv definiten Funktionen, möglich wäre. Wir betrachten Funktionen, die dem Tensorproduktraum univariater Native Spaces entstammen. Dazu wählen wir verschiedene Tensorprodukt-Basisfunktion $\Phi^{i}=\phi_{i_{1}} \otimes \ldots \otimes \phi_{i_{d}}$ mit $i \in \mathbb{N}^{d},|i| \leq q$, wobei die univariaten Basisfunktionen $\phi_{i_{l}}$ bedingt positiv definit bezüglich $\mathcal{P}_{i_{l}}$ sind. Die Funktionen $\Phi^{i}$ seien positiv definit bezüglich $\mathcal{P}_{i_{1}} \otimes \ldots \otimes \mathcal{P}_{i_{d}}$. Zu den univariaten Funktionen $f_{j} \in \mathcal{N}_{\phi_{j}, \mathcal{P}_{j}}\left(\Omega_{j}\right)$ gibt es univariate Basisfunktionen-Interpolanten $S_{\phi_{i_{j}}, X_{i_{j}}} f_{j}=s_{f_{j}, \phi_{i_{j}}, X_{i_{j}}}$ der Gestalt

$$
S_{\phi_{i_{l}}, X_{i_{l}}} f_{l}=\sum_{j_{l}=1}^{N_{i_{l}}} \alpha_{i_{l} j_{l}} \phi_{i_{l}}\left(\cdot, x_{i_{l} j_{l}}\right)+p_{i_{l}}
$$


für die das Gleichungssystem

$$
S_{\phi_{i_{l}}, X_{i_{l}}} f_{l}\left(x_{r_{l} s_{l}}\right)=f_{l}\left(x_{r_{l} s_{l}}\right)
$$

mit $p_{i_{l}} \in \mathcal{P}_{i_{l}}$ und dem Datensatz $X_{i_{l}}=\left\{x_{i_{1} j_{1}}, \ldots, x_{i_{d} j_{d}}\right\}$ für alle $l=1, \ldots, d$, $|i|,|r| \leq q, 1 \leq j_{l} \leq N_{i_{l}}$ und $1 \leq s_{l} \leq N_{r_{l}}$ lösbar ist. Deshalb folgern wir auch die Lösbarkeit der Systeme

$$
\left(S_{\phi_{i_{1}}, X_{i_{1}}} f_{1} \otimes \ldots \otimes S_{\phi_{i_{d}}, X_{i}} f_{d}\right)\left(x_{r_{1} s_{1}}, \ldots, x_{r_{d} s_{d}}\right)=f\left(x_{r_{1} s_{1}}, \ldots, x_{r_{d} s_{d}}\right)
$$

mit $f=f_{1} \otimes \ldots \otimes f_{d}$, welche sich unter Verwendung der Lagrangedarstellungen der Gleichungen auch mittels

$$
\left(S_{\phi_{i_{1}}, X_{i_{1}}} \otimes \ldots \otimes S_{\phi_{i_{d}}, X_{i_{d}}}\right) f\left(x_{r_{1} s_{1}}, \ldots, x_{r_{d} s_{d}}\right)=f\left(x_{r_{1} s_{1}}, \ldots, x_{r_{d} s_{d}}\right)
$$

schreiben lassen; es folgt (20) für alle $f \in \mathcal{N}_{\phi_{i_{1}}, \mathcal{P}_{i_{1}}}\left(\Omega_{i_{1}}\right) \otimes \ldots \otimes \mathcal{N}_{\phi_{i_{d}}, \mathcal{P}_{i_{d}}}\left(\Omega_{i_{d}}\right)$ und schließlich auch auf dem entsprechenden Tensor Native Space

$$
\mathcal{F}_{\phi_{i_{1}}, \mathcal{P}_{i_{1}}}\left(\Omega_{i_{1}}\right) \otimes_{\beta} \ldots \otimes_{\beta} \mathcal{F}_{\phi_{i_{d}}, \mathcal{P}_{i_{d}}}\left(\Omega_{i_{d}}\right)+\mathcal{P}_{\phi_{i_{1}}, \ldots, \phi_{i_{d}}}\left(\Omega_{i_{1}} \times \ldots \times \Omega_{i_{d}}\right) .
$$

Damit gilt auch

$$
\begin{aligned}
& f\left(x_{r_{1} s_{1}}, \ldots, x_{r_{d} s_{d}}\right) \\
& \quad=\sum_{\substack{|i| \leq q \\
i \in N^{d}}}(-1)^{q-|i|}\left(\begin{array}{c}
d-1 \\
q-|i|
\end{array}\right)\left(S_{\phi_{i_{1}}, X_{i_{1}}} \otimes \ldots \otimes S_{\phi_{i_{d}}, X_{i_{d}}}\right) f\left(x_{r_{1} s_{1}}, \ldots, x_{r_{d} s_{d}}\right)
\end{aligned}
$$

aufgrund von (A.4) für $|r| \leq q$ und $1 \leq s_{l} \leq N_{r_{l}}$ für alle $f \in \mathcal{S}_{q, d}^{\mathcal{N}_{\phi_{1}}, \mathcal{P}_{1}, \ldots, \mathcal{N}_{\phi_{d}}, \mathcal{P}_{d}}$. Wir haben damit eine Interpolante an $f$ auf dem dünnen Gitter $H_{q, d}$, welches aus den univariaten Datensätzen $X_{j}=\left\{x_{j 1}, \ldots, x_{j N_{j}}\right\}$ für $1 \leq j \leq q-d+1$ besteht, gefunden.

Da die gleichmäßige Verträglichkeit von Tensorraumnormen nicht unbedingt für Tensorräume mit Seminormen gilt, sind die Fehlerabschätzungen dieses Abschnittes in dieser Form nicht unmittelbar übertragbar. Wir gehen deshalb im folgenden Abschnitt auf eine Methode über, die solche Kern- bzw. Polynomräume benutzt, für die wir eine Aussage treffen können. 


\section{$5 \quad$ Interpolation auf dem gestörten dünnen Gitter}

Neben dem Ansatz in Abschnitt 4 gibt es noch eine weitere Möglichkeit, den SmolyakAlgorithmus für die Interpolation mit Basisfunktionen zu verwenden. Diese Vorgehensweise soll in diesem Abschnitt realisiert werden. Wir gehen von einer Situation aus, wie sie für die klassische Interpolation mit radialen Basisfunktionen entwickelt wurde, vgl. Abschnitt 2. Dabei verwenden wir den Smolyak-Algorithmus bei der Interpolation mit Basisfunktionen so, daß der zugehörige Polynomraum einer SmolyakStruktur folgt. Zu einer allgemeinen, 2d-variaten Basisfunktion $\Phi$ formulieren wir für jede Funktion $f \in \mathcal{N}_{\Phi}$ auf einem $\mathbb{R}^{d}$-wertigen Datensatz $X=\left\{x_{1}, \ldots, x_{n}\right\}$ die Interpolante

$$
s_{f, \Phi, X}=\sum_{j=1}^{n} \alpha_{j} \Phi\left(\cdot, x_{j}\right)+p
$$

mit $\alpha_{1}, \ldots, \alpha_{n} \in \mathbb{R}$ und $p \in \mathcal{S P}_{q, d}^{m_{1}, \ldots, m_{q-d+1}}$, wobei

$$
\sum_{j=1}^{n} \alpha_{j} \tilde{p}\left(x_{j}\right)=0
$$

für alle $\tilde{p} \in \mathcal{S P}_{q, d}^{m_{1}, \ldots, m_{q-d+1}}$ gelte. Ist nun die Basisfunktion $\Phi$ bedingt positiv definit bzgl. des Smolyak-Polynomraumes $\mathcal{S P}_{q, d}^{m_{1}, \ldots, m_{q-d+1}}$, so gibt es immer eine Lösung für das lineare Gleichungssystem. Die Lösung ist nach Satz 2.2 eindeutig, wenn $X$ einen unisolventen Datensatz auf $\mathcal{S P}_{q, d}^{m_{1}, \ldots, m_{q-d+1}}$ enthält bzw. wenn die Matrix $P$ injektiv ist. Die Gleichungssysteme lassen sich für jeden Datensatz $X$ mit $n=|X|=\left|H_{q, d}\right|$ auf eine weitere Weise beschreiben. Wir bezeichnen dabei den Datensatz mittels

$$
X=\left\{x_{i j}\right\}_{\substack{i \in I_{q, d} \\ j \in \tilde{J}_{q, d}}}
$$

mit den Indexmengen

$$
\begin{aligned}
& I_{q, d}=\left\{i=\left(i_{1}, \ldots, i_{d}\right): \quad|i| \leq q\right\} \text { und } \\
& \tilde{J}_{q, d}=\left\{j=\left(j_{1}, \ldots, j_{d}\right): \quad 1 \leq j_{l} \leq m_{i_{l}}, l=1, \ldots, d \text { und } i \in I_{q, d}\right\} .
\end{aligned}
$$

Zudem definieren wir uns eine weitere Indexmenge

$$
K_{\tilde{q}, d}=\left\{k=\left(k_{1}, \ldots, k_{d}\right) \quad: \quad 1 \leq k_{l} \leq \tilde{m}_{i_{l}}, l=1, \ldots, d \text { und } i \in I_{\tilde{q}, d}\right\}
$$


Nun formulieren wir die Interpolante mittels

$$
s_{f, \Phi, X}=\sum_{\substack{i \in I_{q, d} \\ j \in \tilde{J}_{q, d}}} \alpha_{j} \Phi\left(\cdot, x_{i j}\right)+\sum_{\substack{i \in I_{\tilde{q}, d} \\ k \in K_{\tilde{q}, d}}} \beta_{k} p_{k}
$$

mit $p_{k}=p_{k_{1}, \ldots, k_{d}} \in\left(\mathbb{P}_{k_{1}} \otimes \ldots \otimes \mathbb{P}_{k_{d}}\right)$, den reellen Zahlen $\alpha_{j}=\alpha_{j_{1}, \ldots, j_{d}}, \beta_{k}=\beta_{k_{1}, \ldots, k_{d}}$ und

$$
\sum_{\substack{i \in I_{q, d} \\ j \in \tilde{J}_{q, d}}} \alpha_{j} \tilde{p}\left(x_{i j}\right)=0
$$

für alle $\tilde{p} \in \mathcal{S P}_{\tilde{q}, d}^{\tilde{m}_{1}, \ldots, \tilde{m}_{\tilde{q}-d+1}}$. Wir stellen das Gleichungssystem wie auch im klassischen Fall üblich mit Hilfe von Matrizen dar:

$$
\left(\begin{array}{cc}
A_{\Phi, X} & P \\
P^{T} & 0
\end{array}\right)\left(\begin{array}{l}
\alpha \\
\beta
\end{array}\right)=\left(\begin{array}{l}
y \\
0
\end{array}\right) .
$$

Dabei definieren wir die Matrizen $A_{\Phi, X}=\left(\Phi\left(x_{i j}-x_{r s}\right)\right)_{\substack{i, r \in I_{q, d} \\ j, s \in \tilde{J}_{q, d}}}, P_{k}=\left(p_{k}\left(x_{r s}\right)\right)_{\substack{k \in K_{\tilde{q}, d} \\ r \in I_{q, d} \\ s \in \tilde{J}_{q, d}}}$, die Vektoren $\alpha=\left(\alpha_{j}\right)_{j \in \tilde{J}_{q, d}}, \beta=\left(\beta_{k}\right)_{k \in K_{\tilde{q}, d}}$ und $y=\left(f\left(x_{i j}\right)\right)_{\substack{i \in I_{q, d} \\ j \in \tilde{J}_{q, d}}}$. Die Matrix $A_{\Phi, X}$ hat $n(q, d)$ Zeilen und Spalten, wenn wir mit $n(q, d)$ die Anzahl der Punkte des dünnen Gitters $H_{q, d}$ bezeichnen. Die Matrix $P$ besitzt ebenfalls $n(q, d)$ Zeilen; die Anzahl ihrer Spalten entspricht der Dimension $\tilde{n}(\tilde{q}, d)$ des Smolyak-Polynomraumes $\mathcal{S P}_{\tilde{q}, d}^{\tilde{m}_{1}, \ldots, \tilde{m}_{q-d+1}}$. Entsprechend sind $\alpha$ und $y$ Vektoren der Länge $n(q, d)$; der Vektor $\beta$ ist $\tilde{n}(\tilde{q}, d)$-zeilig.

\subsection{Bedingte positive Definitheit im Smolyak-Polynomraum}

Um eine Interpolation mit Basisfunktionen mit Smolyak-Polynomraum durchführen zu können, muß die zugrundegelegte Basisfunktion bedingt positiv definit bezüglich dieses Polynomraumes sein. Eine solche Bedingung erfüllt jede Funktion, die bedingt positiv definit bezüglich eines im Smolyak-Polynomraum enthaltenen Raumes ist. Wir formulieren deshalb:

Lemma 5.1 Seien $\tilde{q}, d, m \in \mathbb{N}$ mit $\tilde{q}=d+\max (0, m-2)$. Dann gilt mit $m_{1} \geq 1$, $m_{j} \geq 2^{j-1}+1$ für $2 \leq j \leq \tilde{q}-d+1$

$$
\mathbb{P}_{m}^{d} \subseteq \mathcal{S P}_{\tilde{q}, d}^{m_{1}, \ldots, m_{\tilde{q}-d+1}}
$$


Beweis: Für $m=1$ kann die Inklusion einfach nachgerechnet werden. Im folgenden sei also $m \geq 2$. Es sei $x^{j}=x_{1}^{j_{1}} \cdot \ldots \cdot x_{d}^{j_{d}}$ ein Basiselement von $\mathbb{P}_{m}^{d}$. An ein Basiselement $x^{j}=x_{1}^{j_{1}} \cdot \ldots \cdot x_{d}^{j_{d}} \in \mathcal{S P}_{\tilde{q}, \boldsymbol{d}}^{m_{1}, \ldots, m_{\tilde{q}-d+1}}$ sind die Nebenbedingungen

$$
\begin{aligned}
& 0 \leq j_{l} \leq m_{i_{l}}-1 \quad \text { und } \\
& i_{1}+\ldots+i_{d} \leq \tilde{q}
\end{aligned}
$$

geknüpft. Von daher ist $x^{j}$ gleichzeitig Basiselement von $\mathcal{S P}_{\tilde{q}, d}^{m_{1}, \ldots, m_{\tilde{q}-d+1}}$, falls $\left(i_{1}, \ldots, i_{d}\right) \in \mathbb{N}_{0}$ existieren mit

$$
\begin{aligned}
& i_{1}+\ldots+i_{d} \leq \tilde{q} \quad \text { und } \\
& 0 \leq j_{l} \leq 2^{i_{l}-1}
\end{aligned}
$$

für alle $1 \leq l \leq d$.

Wäre also $x^{j}$ ein Basiselement von $\mathbb{P}_{m-1}^{d} \subseteq \mathcal{S P} \mathcal{P}_{q(m-1), d}^{m_{1}, \ldots, m_{q(m-1)-d+1}}$, so wäre $x^{\tilde{j}}$ mit $\tilde{j}=$ $j+e_{k}$ und $e_{k}=\left(\delta_{l k}\right)_{1 \leq l \leq d}$ für alle $l$ ein Element der Monombasis des $\mathbb{P}_{m}^{d}$, und jedes Basiselement des $\mathbb{P}_{m}^{d}$ ließe sich auf diese Weise beschreiben. Das Monom $x^{\tilde{j}}$ ist kein Basiselement von $\mathcal{S P}_{q(m-1), d}^{m_{1}, \ldots, m_{q(m-1)-d+1}}$, falls $\tilde{j}_{k}=2^{i_{k}-1}+1$ für $i_{k}>1$ bzw. falls $\tilde{j}_{k}=1$ für $i_{k}=1$ gilt. Dann folgt aber $\tilde{j}_{k} \leq 2^{\left(i_{k}+1\right)-1}$ und $x^{\tilde{j}}$ ist ein Basiselement von $\mathcal{S P}_{q(m-1)+1, d}^{m_{1}, \ldots, m_{q(m-1)-d+2}}$. Daraus schließen wir, daß

$$
\tilde{q} \geq q(m)=q(m-1)+1=\ldots=q(2)+m-2=d+m-2
$$

gelten muß.

Zusammen mit Satz 4.23 folgt

Korollar 5.2 Die stetigen Funktionen $\phi_{j}, 1 \leq j \leq d$, seien bedingt positiv definit der Ordnung $m_{j}$. Dann ist ihre Tensorproduktfunktion $\Phi=\phi_{1} \otimes \ldots \otimes \phi_{d}$ bedingt positiv definit bezüglich des Smolyak-Polynomraumes $\mathcal{S P}_{\tilde{q}, d}^{m_{1}, \ldots, m_{\tilde{q}-d+1}}$ mit $\tilde{q}=d+\max \left(0, \sum_{j=1}^{d} m_{j}-2\right), m_{1} \geq 1$ und $m_{l} \geq 2^{l-1}+1$.

Wie schon im Beweis zu erahnen ist, erhalten wir mit Lemma 5.1 nur eine grobe Schranke. Konkret können wir diesen Sachverhalt etwa im Fall $d=1$ überprüfen, wo offensichtlich $q \geq 1+\max (0, \log (m-1))$ gelten muß. Auch für $d=2$ können wir eine genauere Schranke finden:

Lemma 5.3 Sei $m \in \mathbb{N}, m_{1}=1, m_{j}=2^{j-1}+1,2 \leq j \leq q(m)-1$. Dann gilt

$$
\mathbb{P}_{m}^{2} \subseteq \mathcal{S P}_{q(m), 2}^{m_{1}, \ldots, m_{q(m)-2}}
$$


falls

$$
q(m) \geq \begin{cases}m+1 & : m=1,2 \\ m & : m=3,4,5 \\ 3+\log (m-3) & : m=6,7,8 \\ 1+2 \log (m-2) & : m \geq 9\end{cases}
$$

Beweis: Die Fälle $m=1, \ldots, 5$ können direkt nachgeprüft werden. Ein Basiselement des $\mathbb{P}_{m}^{d} x_{1}^{j_{1}} x_{2}^{j_{2}}$ mit $j_{1}+j_{2} \leq m-1$ ist ebenfalls ein Basiselement von $\mathcal{S P}_{q, d}^{m_{1}, \ldots, m_{q-d+1}}$, falls $i_{1}, i_{2} \in \mathbb{N}_{0}$ existieren mit

$$
\begin{aligned}
i_{1}+i_{2} & =q-2, \\
j_{1} & \leq 2^{i_{1}}-\delta_{i_{1}, 0} \\
j_{2} & \leq 2^{i_{2}}-\delta_{i_{2}, 0} .
\end{aligned}
$$

Von daher betrachten wir im folgenden nur noch Basiselemente des $\mathbb{P}_{m}^{d}$ der Form $x_{1}^{j_{1}} x_{2}^{j_{2}}$ mit $j_{1}+j_{2}=m-1$. Gilt nun $\left(j_{1}, j_{2}\right)=(0, m-1)$, so folgern wir mit $\left(i_{1}, i_{2}\right)=\left(0,2^{q-2}\right)$ wegen $m-2 \geq 3$

$$
\begin{aligned}
& j_{1}=0=2^{i_{1}}-\delta_{i_{1}, 0} \quad \text { und } \\
& j_{2}=m-1 \leq \frac{(m-2)^{2}}{2}=2^{2 \log (m-2)-1} \leq 2^{q(m)-2} .
\end{aligned}
$$

Die letzte Abschätzung folgern wir aus

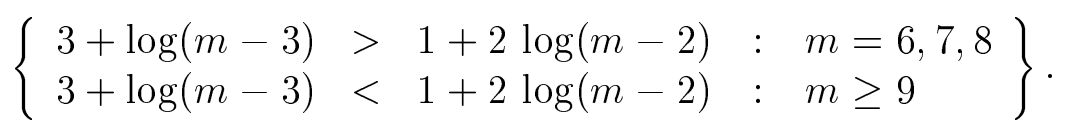

Für die Basiselemente $x_{1}^{j_{1}-1} x_{2}^{j_{2}+1}$ des $\mathbb{P}_{m}^{d}$ gebe es $\left(i_{1}, i_{2}\right) \in \mathbb{N}_{0}$ mit $i_{1}+i_{2}=q-2$,

$$
\begin{aligned}
& j_{1}-1 \leq 2^{i_{1}}-\delta_{i_{1}, 0} \\
& j_{2}+1 \leq 2^{i_{2}}-\delta_{i_{2}, 0} .
\end{aligned}
$$

Wir betrachten nun das Basiselement $x_{1}^{j_{1}} x_{2}^{j_{2}}$. Gilt $j_{1} \leq 2^{i_{1}}-\delta_{i_{1}, 0}$, so haben wir für $\left(j_{1}, j_{2}\right)$ das Zahlenpaar $\left(i_{1}, i_{2}\right)$ gefunden. Ansonsten gilt $j_{1}=2^{i_{1}}+1-\delta_{i_{1}, 0}$. Wir bezeichnen $\tilde{i}_{1}=i_{1}+1$. Es gilt dann

$$
j_{1}=2^{i_{1}}+1-\delta_{i_{1}, 0} \leq 2^{i_{1}+1}=2^{\tilde{i}_{1}} .
$$

Wir wollen nun zeigen, daß

$$
j_{2} \leq 2^{q-2-\tilde{i}_{1}}-\delta_{q-2-\tilde{i}_{1}, 0}=2^{q-3-i_{1}}-\delta_{q-3-i_{1}, 0}
$$


erfüllt ist. Sei zunächst $i_{1}<q-3$. Aufgrund von $j_{2}=m-1-j_{1}=m-2-2^{i_{1}}$ ist die Ungleichung genau dann erfüllt, wenn

$$
m-1=(m-2)+1 \leq 2^{q-3-i_{1}}+2^{i_{1}}+1=\frac{2^{q-3}}{j_{1}-1}+j_{1}
$$

gilt. Wegen

$$
2^{q(m)-3} \geq \frac{(m-2)^{2}}{4} \geq\left(j_{1}-1\right)\left(m-2-j_{1}\right)
$$

ist dieser Sachverhalt durch $q(m)=1+2 \log (m-2)$ gegeben. Letztere Ungleichung erhalten wir, wenn wir die Funktion $f_{m}(x):=(x-1)(m-1-x)$ näher untersuchen. Die Funktion ist auf dem Intervall $\left[1, \frac{m}{2}\right)$ monoton steigend, nimmt an der Stelle $x=\frac{m}{2}$ ihr Maximum an, um dann auf dem Intervall $\left(\frac{m}{2}, m-1\right]$ monoton abzufallen. Im Fall $i_{1}=q-3$ muß die Ungleichung $j_{2} \leq 0$ bzw. $m-1 \leq 2+2^{q-3}$ gelten. Es folgt die Behauptung.

In einem weiteren Spezialfall folgern wir

Lemma 5.4 Gilt $m_{j}=z \cdot j$ für $z \in \mathbb{R}_{\geq 1}$ und $1 \leq j \leq q-\tilde{d}+1$, so ist mit

$$
\tilde{q} \geq \max (d, d+m-z)
$$

der klassische Polynomraum $\mathbb{P}_{m}^{d}$ im Smolyak-Polynomraum $\mathcal{S P}_{\tilde{q}, d}^{m_{1}, \ldots, m_{\tilde{q}-d+1}}$ enthalten.

Beweis: Unter Beachtung von $\mathbb{P}_{z}^{d} \subseteq \mathcal{S P}_{d, d}^{m_{1}}$ gehen wir analog zum Beweis von Lemma 5.1 vor.

Ein weiteres Resultat erhalten wir, wenn wir Smolyak-Funktionen, bestehend aus univariaten positiv definiten Funktionen, betrachten:

Lemma 5.5 Es seien $\phi_{j}, 1 \leq j \leq q-d+1$, paarweise verschiedene, stetige, positiv definite Funktionen mit verallgemeinerten Fouriertransformierten $\hat{\phi}_{j}$, für die die Ungleichung $\hat{\phi}_{j}(\omega) \geq \hat{\phi}_{j-1}(\omega)$ für alle $\omega \neq 0$ erfüllt sei. Dann ist die Smolyakfunktion $A_{q_{2} d}\left(\phi_{1}, \ldots, \phi_{q-d+1}\right)$ bedingt positiv definit bezüglich des Smolyak-Polynomraumes $\mathcal{S P}_{q, d}^{\tilde{m}_{1}, \ldots, \tilde{m}_{q-d+1}}$ mit $\tilde{m}_{1}:=m_{1}$ und $\tilde{m}_{j}=\max \left(m_{j}, m_{j-1}\right)$ für $j \geq 2$. 


\section{Beweis:}

Da $\hat{\phi}_{j}$ um Null integrierbar ist, folgern wir dieselbe Eigenschaft für die Funktion $\hat{\phi}_{j}-\hat{\phi}_{j-1}$ und es gilt wegen $\hat{\phi}_{j}-\hat{\phi}_{j-1} \geq 0$ mit dem Satz 2.4 von Iske die positive Definitheit von $\phi_{j}-\phi_{j-1}$. Aufgrund von Satz 4.23 sind also die Funktionen

$$
\left(\phi_{j_{1}}-\phi_{j_{1}-1}\right) \otimes \ldots \otimes\left(\phi_{j_{d}}-\phi_{j_{d}-1}\right)
$$

positiv definit. Damit folgt

$$
\begin{aligned}
& \sum_{l=1}^{n} \sum_{k=1}^{n} \alpha_{l} \alpha_{k} A_{q, d}\left(\phi_{1}, \ldots, \phi_{q-d+1}\right)\left(x_{l}, x_{k}\right) \\
& \quad=\sum_{\substack{|j|_{1, d} \leq q \\
j \in \mathbb{N}^{d}}} \sum_{l=1}^{n} \sum_{k=1}^{n} \alpha_{l} \alpha_{k}\left(\phi_{j_{1}}-\phi_{j_{1}-1}\right) \otimes \ldots \otimes\left(\phi_{j_{d}}-\phi_{j_{d}-1}\right)\left(x_{l}, x_{k}\right)>0 .
\end{aligned}
$$

\subsection{Das gestörte dünne Gitter}

In diesem Abschnitt werden wir klären, welchen chaotisch verteilten Datensatz wir verwenden wollen. Wir werden diesen Datensatz ein gestörtes dünnes ClenshawCurtis-Gitter nennen und beziehen uns dabei sowohl auf das klassische ClenshawCurtis-Gitter $H_{q, d}^{C C}$ mit $m_{1}=1, m_{j}=2^{j-1}+1,2 \leq j \leq q-d+1$, als auch auf das dünne Gitter $H_{q, d}^{C C 2}$ mit $m_{j}=2^{j-1}+1,1 \leq j \leq q-d+1$. In Analogie zum gestörten vollbesetzten Gitter bzw. quasi-regulärem Datensatz formulieren wir:

Definition 5.6 Sei $\Omega$ kompakt, sei $q \geq d$. Wir nennen den Datensatz $X \subset \Omega$ mit $|X|=\left|H_{q, d}^{C C}\right|$ oder $|X|=\left|H_{q, d}^{C C 2}\right|$ ein gestörtes (dünnes) Clenshaw-Curtis-Gitter auf $\Omega$, falls er für

$$
\begin{aligned}
\check{q} & =\min _{\substack{x, \tilde{x} \in X \\
x \neq \tilde{x}}}\|x-\tilde{x}\| \quad \text { und } \\
h & =h_{X, \Omega}=\sup _{y \in \Omega} \min _{x \in X}\|x-y\|
\end{aligned}
$$

die Bedingung

$$
\check{q} \leq h \leq c_{1} d 2^{(q-d) \frac{d-1}{d}} \check{q}
$$

mit $c_{1} \in \mathbb{R}_{+}$erfüllt. Wir bezeichnen $X$ mit $L_{q, d}^{C C}$ bzw. $L_{q, d}^{C C 2}$. 
Ein Clenshaw-Curtis-Gitter ist unter folgender Bedingung auch gleichzeitig ein gestörtes:

Satz 5.7 Sei $H_{q, d}^{C C}$ ein Clenshaw-Curtis-Gitter mit $q>d$, und sei $H_{q, d}^{C C 2}$ ein dünnes Gitter mit $m_{j}=2^{j-1}+1$. Bei beiden dünnen Gittern seien die univariaten Datensätze $X_{j}=\left\{x_{j 1}, \ldots, x_{j m_{j}}\right\}, 1 \leq j \leq q-d+1$, verschachtelt, es gelte

$$
x_{j+1 k}=\frac{x_{j\left\lfloor\frac{k+1}{2}\right\rfloor}+x_{j\left\lceil\frac{k+1}{2}\right\rceil}}{2}
$$

im Clenshaw-Curtis-Fall sei $x_{12}:=x_{11}$. Dann sind beide Gitter gestörte ClenshawCurtis-Gitter.

Bedingung (23) bedeutet, daß die Datenpunkte des univariaten Datensatzes $X_{j+1} \backslash X_{j}$ genau zwischen den Punkten des Datensatzes $X_{j}$ liegen. Die Daten sind damit äquidistant verteilt, und die Schrittweite $\left|x_{j k}-x_{j k+1}\right|, 2 \leq j \leq q-d+1,1 \leq k \leq 2^{j-1}$, halbiert sich mit jeder Verfeinerung des Datensatzes.

Beweis: Die Ungleichung $\check{q} \leq h_{H_{q, d}^{C C}, \Omega}$ ist trivial. Wir benennen im folgenden beide dünnen Gitter mit $H_{q, d}$. Bezeichne $G_{q, d}$ das vollbesetzte Gitter mit

$$
G_{q, d}=X_{q-d+1} \times \ldots \times X_{q-d+1}
$$

es erfüllt

$$
h_{G_{q, d}, \Omega} \leq \check{q}
$$

Sei $\tilde{x} \in G_{q, d}$ der Gitterpunkt, für den

$$
h_{G_{q, d}, \Omega}=\sup _{y \in \Omega}\|\tilde{x}-y\|
$$

angenommen wird. Offenbar gilt $\tilde{x}=\left(\tilde{x}_{1}, \ldots, \tilde{x}_{d}\right) \in X_{l_{1}} \times \ldots \times X_{l_{d}}$ mit $d \leq|l|_{d} \leq d(q-d+1)$. Wir wählen nun einen Punkt $B_{l_{j}}\left(\tilde{x}_{j}\right)=: x_{j}$ nach folgender Abbildungsvorschrift: Für $\tilde{x}_{j} \in X_{l_{j}} \backslash X_{l_{j}-1}$ sei $x_{j}=x_{l_{j}-1 k} \in \mathbb{R}$ der Datenpunkt aus $X_{l_{j}-1}$, für den $\tilde{x}_{j}$ Mittelpunkt der Strecke zwischen $x_{l_{j}-1 k}$ und einem Nachbarpunkt $x_{l_{j}-1 k-1}$ oder $x_{l_{j}-1 k+1}$ darstellt. Im Clenshaw-Curtis-Fall nehmen wir den Nachbarpunkt $x_{l_{j}-1 k-1}$ falls $k \geq 2^{l_{j}-3}+1$; andernfalls entscheiden wir uns für den Nachbarpunkt $x_{l_{j}-1 k+1}$. Im Fall des zweiten dünnen Gitters $H_{q, d}^{C C 2}$ wählen wir genau umgekehrt. Nun definieren wir die Abbildung

$$
B_{l i}:=B_{i+1} \circ B_{i+2} \circ \ldots \circ B_{l}
$$


die komponentenweise Anwendung dieser Punktauswahl wird mit Hilfe des Symbols $\otimes$ formuliert. Wir folgern mit $z=\left(B_{l_{1} i_{1}} \otimes \ldots \otimes B_{l_{d} i_{d}}\right) \tilde{x} \in X_{i_{1}} \times \ldots \times X_{i_{d}}$ für $|i|_{d} \leq q$

$$
\begin{aligned}
h_{H_{q, d}, \Omega} & \leq \sup _{y \in \Omega}\|z-y\| \\
& \leq \sup _{y \in \Omega}(\|z-\tilde{x}\|+\|\tilde{x}-y\|) \\
& \leq\|z-\tilde{x}\|+\frac{\sqrt{d}}{2} \check{q} .
\end{aligned}
$$

Jetzt untersuchen wir

$$
\|z-\tilde{x}\|=\left\|\left(B_{l_{1} i_{1}} \otimes \ldots \otimes B_{l_{d} i_{d}}\right) \tilde{x}-\tilde{x}\right\| .
$$

Bezeichnen wir mit $I_{1}$ die Identitätsabbildung, so gilt für entsprechende $\tilde{z}$ offenbar

$$
\left\|\left(I_{1} \otimes \ldots \otimes I_{1} \otimes B_{l} \otimes I_{1} \otimes \ldots \otimes I_{1}\right) \tilde{z}-\tilde{z}\right\| \leq 2^{q-d+1-l} \check{q} .
$$

Damit folgern wir für $l>i$ unter Verwendung der Dreiecksungleichung

$$
\begin{aligned}
\left\|\left(I_{1} \otimes \ldots \otimes I_{1} \otimes B_{l i} \otimes I_{1} \otimes \ldots \otimes I_{1}\right) \tilde{z}-\tilde{z}\right\| & \leq \sum_{\mu=i+1}^{l} 2^{q-d+1-\mu} \check{q} \\
& =2^{q-d+1-l} \sum_{\mu=0}^{l-i-1} 2^{\mu} \\
& =2^{q-d+1-l}\left(2^{l-i}-1\right) \\
& \leq 2^{q-d+1-i}
\end{aligned}
$$

und schließlich für $l_{j}>i_{j}, 1 \leq j \leq d$, wieder mit Hilfe der Dreiecksungleichung

$$
\left\|\left(B_{l_{1} i_{1}} \otimes \ldots \otimes B_{l_{d} i_{d}}\right) \tilde{x}-\tilde{x}\right\| \leq \sum_{j=1}^{d} 2^{q-d+1-i_{j}} .
$$

Wir wählen nun $i_{1}=\ldots=i_{d\left(1+\left\lfloor\frac{q}{d}\right\rfloor\right)-q}=\left\lfloor\frac{q}{d}\right\rfloor$ und $i_{d\left(1+\left\lfloor\frac{q}{d}\right\rfloor\right)-q+1}=\ldots=i_{d}=1+\left\lfloor\frac{q}{d}\right\rfloor$. Es gilt dann

$$
\begin{aligned}
\| & \left(B_{l_{1} i_{1}} \otimes \ldots \otimes B_{l_{d} i_{d}}\right) \tilde{x}-\tilde{x} \| \\
& \leq\left(\left(d\left(1+\left\lfloor\frac{q}{d}\right\rfloor\right)-q\right) 2^{q-d+1-\left\lfloor\frac{q}{d}\right\rfloor}+\left(q-\left\lfloor\frac{q}{d}\right\rfloor d\right) 2^{\left.q-d+1-\left(1+\left\lfloor\frac{q}{d}\right\rfloor\right)\right) \check{q}}\right. \\
& \leq\left(2 d\left(1+\left\lfloor\frac{q}{d}\right\rfloor\right)-2 q+\left(q-\left\lfloor\frac{q}{d}\right\rfloor d\right)\right) 2^{q-d-\left\lfloor\frac{q}{d}\right\rfloor} \check{q} \\
& \leq\left(2 d+d\left\lfloor\frac{q}{d}\right\rfloor-q\right) 2^{q-d-\frac{q}{d}+1} \check{q} \\
& \leq d 2^{(q-d) \frac{d-1}{d}+1} \check{q}
\end{aligned}
$$


und wir folgern

$$
h_{H_{q, d}, \Omega} \leq\left(d 2^{(q-d) \frac{d-1}{d}+1}+\frac{\sqrt{d}}{2}\right) \check{q} \leq \frac{5}{2} d 2^{(q-d) \frac{d-1}{d}} \check{q} .
$$

Auf einem solchen gestörten dünnen Gitter gilt:

Satz 5.8 Sei $\Omega$ beschränkt. Ist $X=L_{q, d}^{C C}$ bzw. $L_{q, d}^{C C 2}$ ein gestörtes dünnes Gitter auf $\Omega$, so folgern wir mit $\check{c} \in \mathbb{R}_{+}$

$$
h_{X, \Omega} \leq \check{c} \frac{2^{\frac{1}{d}} d}{(d-1) ! \frac{1}{d}}(2 d+\log (n))^{\frac{d-1}{d}} n^{-\frac{1}{d}} .
$$

Dabei bezeichnet $n$ die Anzahl der Punkte des Datensatzes.

Beweis: Sei $N_{q, d}=2^{(q-d+1) d}$ die Anzahl der Punkte auf dem vollbesetzten Gitter $G_{q, d}=X_{q-d+1} \times \ldots \times X_{q-d+1}$. Aufgrund der Kompaktheit von $\Omega$ schlußfolgern wir $N \check{q}^{d} \leq C$. Das ist einzusehen, wenn man um jeden Gitterpunkt von $G_{q, d}$ eine $d$-dimensionale Kugel mit Radius $\frac{\breve{q}}{2}$ legt und dann das Volumen dieser Kugelmenge gegen das von $\Omega$ abschätzt. Es gilt also

$$
\check{q} \leq C N^{-\frac{1}{d}}=C 2^{-(q-d+1)}
$$

und wir folgern unter Nutzung der Korollare 3.29 und 3.31

$$
\begin{aligned}
h_{X, \Omega} & \leq c_{1} d 2^{(q-d) \frac{d-1}{d}} \check{q} \\
& \leq c_{3} d 2^{-\frac{q}{d}} \\
& \leq 2 c_{3} \frac{2^{\frac{1}{d}} d}{(d-1) ! \frac{1}{d}} n^{-\frac{1}{d}}(2 d+\log (n))^{\frac{d-1}{d}} .
\end{aligned}
$$

Vergleichen wir die Fülldistanz eines vollbesetzten Gitters mit der Fülldistanz eines gestörten dünnen Gitters bei gleicher Punktanzahl, so erhalten wir folgendes Ergebnis: 
Korollar 5.9 Es gelten die Voraussetzungen von Satz 5.8. Dann folgt für den Quotienten der Fülldistanz $h_{X, \Omega}$ eines gestörten dünnen Gitters $X$ und der Fülldistanz $h_{G_{d}, \Omega}$ eines vollbesetzten d-dimensionalen Gitters $G_{d}$ bei fester Punktanzahl $|X|=\left|G_{d}\right|=n$ die Ungleichung

$$
\frac{h_{X, \Omega}}{h_{G_{d}, \Omega}} \leq \mathcal{O}(d), \quad d \rightarrow \infty
$$

Beweis: Wegen $h_{G_{d}, \Omega} \approx n^{-\frac{1}{d}}$ betrachten wir den Faktor

$$
K_{n}(d):=\frac{2^{\frac{1}{d}} d}{(d-1) ! !^{\frac{1}{d}}}(2 d+\log (n))^{\frac{d-1}{d}} .
$$

Unter Verwendung der Stirlingschen Formel folgt für große Werte von $d$

$$
\begin{aligned}
\frac{2^{\frac{1}{d}} d}{(d-1) ! !^{\frac{1}{d}}} & =\frac{2^{\frac{1}{d}} d^{1+\frac{1}{d}}}{d !^{\frac{1}{d}}}=\mathcal{O}\left(\frac{2^{\frac{1}{d}} d^{1+\frac{1}{d}}}{(2 \pi d)^{\frac{1}{2 d}} d e^{-1}}\right)=\mathcal{O}\left(\left(2 d \pi^{-1}\right)^{\frac{1}{2 d}}\right) \\
& =\mathcal{O}\left(\exp \left(\frac{1}{2 d} \ln \left(2 d \pi^{-1}\right)\right)\right)=\mathcal{O}(1), \quad d \rightarrow \infty
\end{aligned}
$$

es gilt auch

$$
(2 d+\log (n))^{\frac{1}{d}}=\exp \left(\frac{1}{d} \ln (2 d+\log (n))\right)=\mathcal{O}(1), d \rightarrow \infty
$$

und zusammengefaßt folgern wir $K_{n}(d)=\mathcal{O}(d), d \rightarrow \infty$.

Im folgenden Unterabschnitt werden zur Bestimmung des Interpolationsfehlers vorbereitende Ergebnisse ermittelt.

\subsection{Fehlerabschätzungen}

Bei der Fehlerabschätzung im Rahmen der Interpolation mit Basisfunktionen wird darauf abgezielt, eine obere Schranke für die entsprechende Gütefunktion zu finden. Dabei wird, wie bereits in Abschnitt 2 erwähnt, auf Ergebnisse der Polynominterpolation zurückgegriffen. Deshalb notieren wir an dieser Stelle einige Folgerungen aus Lemma 4.28, die für derartige Zwecke nutzbar sind. Wie auch im klassischen Fall formulieren wir aufgrund der Stetigkeit der Determinante 
Korollar 5.10 Für die Punkte des speziellen dünnen Gitters

$$
H_{q, d}^{\mathbb{Z}}=\bigcup_{|i|_{d}=q}\left(X_{i_{1}}^{\mathbb{Z}} \otimes \cdots \otimes X_{i_{d}}^{\mathbb{Z}}\right)
$$

mit $X_{i}^{\mathbb{Z}}=\left\{0, \ldots, m_{i}-1\right\}$ gilt

(1) $\operatorname{det}\left(k^{l}\right)_{k, l \in H_{q, d}^{\mathbb{Z}}} \neq 0$ und

(2) es existiert $\epsilon_{0}>0, \epsilon_{0} \in \mathbb{R}$, so daß für alle $x_{k}$ mit $\left\|x_{k}-k\right\| \leq \epsilon_{0}$ und $k \in H_{q, d}^{\mathbb{Z}}$ die Polynominterpolation bezüglich $\mathcal{S P}_{q, d}^{m_{1}, \ldots, m_{q-d+1}}$ eindeutig lösbar ist.

Lemma 5.11 Für alle $q \geq d, m_{l} \geq 1,1 \leq l \leq q-d+1$ und zu jedem $\zeta>0$ existieren $h_{0}>0, c_{1}, c_{2}>0$, so daß es für alle $X=\left\{x_{1}, \ldots, x_{N}\right\} \subset \mathbb{R}^{d}$ und

$$
h_{\zeta}(x):=\sup _{\|y-x\| \leq \zeta} \min _{z \in X}\|z-y\| \leq h_{0}
$$

$\tilde{u}_{1}(x), \ldots, \tilde{u}_{N}(x) \in \mathbb{R}$ gibt mit

(1) $p(x)=\sum_{j=1}^{N} \tilde{u}_{j}(x) p\left(x_{j}\right)$ für alle $p \in \mathcal{S P}_{q, d}^{m_{1}, \ldots, m_{q-d+1}}$,

(2) $\tilde{u}_{j}(x)=0$ für alle $j$ mit $\left\|x_{j}-x\right\|>c_{1} h_{\zeta}(x)$ und

(3) $\sum_{j=1}^{N}\left|\tilde{u}_{j}(x)\right| \leq c_{2}$.

Beweis: Sei $\alpha \in H_{q, d}^{\mathbb{Z}}$ gegeben. Bezeichne $y_{\alpha}:=x+\alpha c_{0} h_{\zeta}(x)$. Es gilt

$$
\begin{aligned}
\left\|y_{\alpha}-x\right\| & =\left\|\alpha c_{0} h_{\zeta}(x)\right\|=|\alpha|\left\|c_{0} h_{\zeta}(x)\right\| \leq \max _{\substack{1 \leq j \leq d \\
i_{1}+\cdots+i_{d}=q}}\left(m_{i_{j}}-1\right)\left\|c_{0} h_{\zeta}(x)\right\| \\
& \leq m_{q, d} c_{0} h_{0}=m_{q, d} c_{0} \frac{\zeta}{m_{q, d} c_{0}}=\zeta
\end{aligned}
$$

mit $m_{q, d}:=\max _{i_{1}+\ldots+i_{d}=q}\left(m_{i_{j}}-1\right)$ und $h_{0}:=\frac{\zeta}{m_{q, d} c_{0}}$. Damit folgt $h_{\zeta}(x) \geq \min _{z \in X}\left\|y_{\alpha}-z\right\|$ und $\mathrm{zu}$ jedem $\alpha \in H_{q, d}^{\mathbb{Z}}$ existiert $z_{\alpha} \in X$ mit $\left\|y_{\alpha}-z_{\alpha}\right\| \leq h_{\zeta}(x)$. In diesen Punkten $\left\{z_{\alpha}\right\}_{\alpha \in H_{q, d}^{\mathbb{Z}}}$ ist eine Polynominterpolation möglich, denn mit $w_{\alpha}:=z_{\alpha}-x$ gilt $\left\|w_{\alpha}-\alpha c_{0} h_{\zeta}(x)\right\| \leq h_{\zeta}(x) \leq h_{0}$ und folglich $\left\|\frac{w_{\alpha}}{c_{0} h_{\zeta}(x)}-\alpha\right\| \leq \frac{1}{c_{0}}$. Unter Anwendung von Korollar 5.10 kann also in den Punkten $\frac{w_{\alpha}}{c_{0} h_{\zeta}(x)}{ }_{\alpha \in H_{q, d}^{\mathbb{Z}}}^{\mathbb{Z}}$ auf dem Polynomraum $\mathcal{S P}_{q, d}^{m_{1}, \ldots, m_{q-d+1}}$ mit $\epsilon_{0}=\frac{1}{c_{0}}$ interpoliert werden. 
Wählen wir also eine Lagrangebasis $\left\{u_{\beta}(x)\right\}_{\beta \in H_{q, d}^{\mathbb{Z}}} \in \mathcal{S P}_{q, d}^{m_{1}, \ldots, m_{q-d+1}}$ mit $u_{\beta}\left(\frac{w_{\alpha}}{c_{0} h_{\zeta}(x)}\right)=\delta_{\alpha, \beta}$, so ist $u_{\beta}\left(\frac{\cdot}{c_{0} h_{\zeta}(x)}\right)$ Lagrangebasis zu $\left\{w_{\alpha}\right\}_{\alpha \in H_{q, d}^{\mathbb{Z}}}$ und damit erhalten wir eine Lagrangebasis $u_{\beta}\left(\frac{\cdot-x}{c_{0} h_{\zeta}(x)}\right) \mathrm{zu}\left\{z_{\alpha}\right\}_{\alpha \in H_{q, d}^{\mathbb{Z}}}$. Nun sind wir in der Lage, die im Lemma beschriebene Lagrangebasis zu definieren. Wir bezeichnen

$$
\tilde{u}_{l}(y):=u_{\beta}\left(\frac{y-x}{c_{0} h_{\zeta}(x)}\right) \delta_{x_{l}, z_{\beta}}
$$

mit $1 \leq l \leq N, y \in \mathbb{R}^{d}$ und $\beta \in H_{q, d}^{\mathbb{Z}}$. Hiermit gilt für alle $p \in \mathcal{S P}_{q, d}^{m_{1}, \ldots, m_{q-d+1}}$

$$
\begin{aligned}
p(y) & =\sum_{\substack{0 \leq \beta_{j} \leq m_{i_{j}}-1 \\
\beta=\left(\beta_{1}, \ldots, \beta_{d}\right) \in \mathbb{N}_{0}^{d} \\
|i|_{d} \leq q}} u_{\beta}\left(\frac{y-x}{c_{0} h_{\zeta}(x)}\right) p\left(z_{\beta}\right) \\
& =\sum_{l=1}^{N} \tilde{u}_{l}(y) p\left(x_{l}\right) .
\end{aligned}
$$

Weiterhin schließen wir vom Nichtverschwinden von $\tilde{u}_{l}(y)$ auf $x_{l}=z_{\beta}$; von daher ist die Ungleichung

$$
\left\|x-\beta c_{0} h_{\zeta}(x)-x_{l}\right\| \leq h_{\zeta}(x)
$$

erfüllt. Damit folgt auch

$$
\left\|x-x_{l}\right\| \leq h_{\zeta}(x)+|\beta| c_{0} h_{\zeta}(x) \leq h_{\zeta}(x) c_{1}
$$

wobei $c_{1}:=\left(1+c_{0} m_{q, d}\right)$ definiert ist. Zudem gilt

$$
\sum_{l=1}^{N}\left|\tilde{u}_{l}(x)\right|=\sum_{l=1}^{N}\left|u_{\beta}(0)\right| \delta_{x_{l}, z_{\beta}}=\sum_{\beta \in H_{q, d}^{\mathbb{Z}}}\left|u_{\beta}(0)\right| \leq c_{2}
$$

mit $c_{2} \in \mathbb{R}_{+}$. Zuletzt muß noch die Eindeutigkeit der Darstellung der Datenpunkte angemerkt werden; aus $x_{l}=z_{\alpha}=z_{\beta}$ und der Wahl von $c_{0}>2$ folgt

$$
\left\|x+\alpha c_{0} h_{\zeta}(x)-z_{\alpha}\right\|+\left\|x+\beta c_{0} h_{\zeta}(x)-z_{\beta}\right\| \leq 2 h_{\zeta}(x)
$$

und deshalb $\left\|(\alpha-\beta) c_{0} h_{\zeta}(x)\right\| \leq 2 h_{\zeta}(x)<1$.

Bemerkung 5.12 Die Konstanten $c_{1}$ und $c_{2}$ aus Lemma 5.11 unterscheiden sich deutlich von denen aus Lemma 2.8. 


\subsubsection{Allgemeine Basisfunktionen}

Nun wollen wir die Gütefunktion im allgemeinen Fall abschätzen.

Satz 5.13 Seien $q, \tilde{q}, d, k, \tilde{m}_{1}, \ldots, \tilde{m}_{\tilde{q}-d+1}, m_{1}, \ldots, m_{q-d+1} \in \mathbb{N}$ natürliche Zahlen, welche die Bedingungen $\mathcal{P} \subseteq \mathcal{S P}_{\tilde{q}, d}^{\tilde{m}_{1}, \ldots, \tilde{m}_{\tilde{q}-d+1}}, \tilde{m}_{j} \leq m_{j}$ und $q \geq \tilde{q}, k \geq d$ für einen $d$-variaten Polynomraum $\mathcal{P}$ erfüllen. Dann gilt für eine $d$-variate, bezüglich $\mathcal{P}$ bedingt positiv definite Basisfunktion $\Phi$, für jeden Datensatz $X=\left\{x_{1}, \ldots, x_{N}\right\}$ mit $N=\left|H_{q, d}^{C C}\right|$ oder $\left|H_{q, d}^{C C 2}\right|$ und jeden Punkt $x \in \mathbb{R}^{d}$ mit

$$
h_{\zeta}(x)=\sup _{\|y-x\| \leq \zeta} \min _{z \in X}\|z-y\| \leq h_{0}=\frac{\zeta \epsilon_{0}}{m_{q, d}}
$$

und $\epsilon_{0}<\frac{1}{2}$ die Abschätzung

$$
P_{X, \tilde{u}, x}^{2} \leq\left(1+c_{2}\right)^{2} E_{d}^{\mathcal{S}}(k, \Phi, \epsilon)
$$

mit $\epsilon \geq 2 c_{1} h_{\zeta}(x), c_{1}=1+\frac{m_{q, d}}{\epsilon_{0}}$, der ebenfalls von $\epsilon_{0}$ und $m_{q, d}=\max _{|i|_{d}=q}\left(m_{i_{j}}-1\right)$ abhängigen Lebesgueschen Konstanten $c_{2} \in \mathbb{R}_{+}$und dem Approximationsfehler

$$
E_{d}^{\mathcal{S}}(k, \Phi, \epsilon):=\inf _{p \in \mathcal{S} \mathcal{P}_{k, d}^{m_{1}, \ldots, m_{k-d+1}}} \max _{\|x\| \leq \epsilon}|\Phi(x)-p(x)| .
$$

Beweis: Wir bezeichnen die Lösung der Approximationsaufgabe (25) mit $p_{k, d}^{\mathcal{S}, *}$ und definieren für alle $y \in \mathbb{R}^{d}$

$$
Q_{x, \tilde{u}}^{\mathcal{S}}(y):=p_{k, d}^{\mathcal{S}, *}(x-y)-\sum_{j=1}^{N} \tilde{u}_{j}(x) p_{k, d}^{\mathcal{S}, *}\left(x_{j}-y\right) .
$$

Wegen $k \leq q$ wird $p_{k, d}^{\mathcal{S}, *}(x-y)$ durch die Lagrangefunktionen exakt reproduziert und es gilt $Q_{X, \tilde{u}}^{\mathcal{S}}(y)=0$. Daraus können wir

$$
\begin{aligned}
0 & =Q_{x, \tilde{u}}^{\mathcal{S}}(x)-\sum_{j=1}^{N} Q_{x, \tilde{u}}^{\mathcal{S}}\left(x_{j}\right) \\
& =p_{k, d}^{\mathcal{S}, *}(0)-2 \sum_{j=1}^{N} \tilde{u}_{j}(x) p_{k, d}^{\mathcal{S}, *}\left(x_{j}-x\right)+\sum_{i, j=1}^{N} \tilde{u}_{j}(x) p_{k, d}^{\mathcal{S}, *}\left(x_{i}-x_{j}\right)
\end{aligned}
$$


schließen. Wir betrachten nun

$$
\begin{aligned}
P_{X, \tilde{u}, x}^{2}= & \Phi(0)-2 \sum_{j=1}^{N} \tilde{u}_{j}(x) \Phi\left(x_{i}-x\right)+\sum_{i, j=1}^{N} \tilde{u}_{i}(x) \tilde{u}_{j}(x) \Phi\left(x_{i}-x_{j}\right) \\
= & \left(\Phi(0)-p_{k, \boldsymbol{d}}^{\mathcal{S}, *}(0)\right)-\left(2 \sum_{j=1}^{N} \tilde{u}_{j}(x) \Phi\left(x_{i}-x\right)-2 \sum_{j=1}^{N} \tilde{u}_{j}(x) p_{k, d}^{\mathcal{S}, *}\left(x_{i}-x\right)\right)+ \\
& +\left(\sum_{i, j=1}^{N} \tilde{u}_{i}(x) \tilde{u}_{j}(x) \Phi\left(x_{i}-x_{j}\right)-\sum_{i, j=1}^{N} \tilde{u}_{i}(x) \tilde{u}_{j}(x) p_{k, d}^{\mathcal{S}, *}\left(x_{i}-x_{j}\right)\right) .
\end{aligned}
$$

Unter Anwendung von Teil (2) von Lemma 5.11 folgern wir wegen

$$
\epsilon \geq 2 c_{1} h_{\zeta}(x) \geq 2 \max _{\substack{1 \leq j \leq N \\ \tilde{u}_{j}(x) \neq 0}}\left\|x_{j}-x\right\| \geq\left\|x_{i}-x\right\|+\left\|x_{j}-x\right\|
$$

und genauso $\epsilon \geq\left\|x_{j}-x\right\|$ für alle $i, j$ mit $\tilde{u}_{i}(x), \tilde{u}_{j}(x) \neq 0$ die Abschätzung

$$
\begin{aligned}
P_{X, \tilde{u}, x}^{2} & \leq E_{d}^{\mathcal{S}}(k, \Phi, \epsilon)+2 \sum_{j=1}^{N}\left|\tilde{u}_{j}(x)\right| E_{d}^{\mathcal{S}}(k, \Phi, \epsilon)+\left(\sum_{j=1}^{N}\left|\tilde{u}_{j}(x)\right|\right)^{2} E_{d}^{\mathcal{S}}(k, \Phi, \epsilon) \\
& \leq\left(1+2 c_{2}+c_{2}^{2}\right) E_{d}^{\mathcal{S}}(k, \Phi, \epsilon)=\left(1+c_{2}\right)^{2} E_{d}^{\mathcal{S}}(k, \Phi, \epsilon) .
\end{aligned}
$$

Durch Anwendung von Satz 4.29 erhalten wir für bestimmte Basisfunktionen eine konkretere Fehlerschranke.

Satz 5.14 Sei $\mathcal{F} \subseteq C^{\nu}(\Omega)$ mit $\Omega \subseteq \mathbb{R}$ ein Banachraum. Das dünne Gitter $H_{q, d}^{C C}$ mit den Gitterpunkten $x_{i_{l} j_{l}}=-\cos \left(\pi\left(j_{l}-1\right)\left(m_{i_{l}}-1\right)^{-1}\right)$ für $1 \leq j_{l} \leq m_{i_{l}}$, $i=\left(i_{1}, \ldots, i_{d}\right) \in \mathbb{I N}^{d}, k-d+1 \leq|i| \leq k$ und $1 \leq l \leq d$ sei in $\Omega$ enthalten. Unter den Voraussetzungen von Satz 5.13 gilt für $h \leq 1,[0, h] \subseteq \Omega$ und $\Phi \in \mathcal{F} \otimes \ldots \otimes \mathcal{F}$ mit Tensorraumnorm $\|\cdot\|_{\mathcal{F} \otimes \ldots \otimes \mathcal{F}}$

$$
E_{d}^{\mathcal{S}}(k, \Phi, h) \leq c d \max \left(2 c, c_{\nu}\right)^{d-1} 2^{-\nu k} k^{2 d-1}\|\Phi\|_{\mathcal{F} \otimes \ldots \otimes \mathcal{F}}
$$

mit $c_{\nu}=1 \mathrm{im}$ Clenshaw-Curtis-Fall und $c_{\nu}=2^{\nu}$ im Fall $m_{j}=2^{j-1}+1$, $1 \leq j \leq q-d+1$

Beweis: Es gilt

$$
\inf _{p \in \mathcal{S P}_{k, d}^{m_{1}, \ldots, m_{k-d+1}}} \max _{\|x\| \leq h}|\Phi(x)-p(x)| \leq \max _{\|x\| \leq h}|\Phi(x)-\tilde{p}(x)|
$$


für alle $\tilde{p} \in \mathcal{S P}_{k, d}^{m_{1}, \ldots, m_{k-d+1}}$, also auch, falls wir

$$
\begin{aligned}
\tilde{p}(x)=\tilde{p}_{\Phi}(x) & =\sum_{\substack{1 \leq j_{l} \leq m_{i_{l}} \leq k \\
k-d+1 \leq\left. i\right|_{1, d} \leq k}}(-1)^{k-|i|_{d}}\left(\begin{array}{c}
d-1 \\
k-|i|_{1, d}
\end{array}\right) \Phi\left(x_{i_{1}, j_{1}}, \ldots, x_{i_{d}, j_{d}}\right) x^{j} \\
& =\sum_{k-d+1 \leq|i|_{1, d} \leq k}(-1)^{k-|i|_{d}}\left(\begin{array}{c}
d-1 \\
k-|i|_{1, d}
\end{array}\right) \mathbf{U}_{i, d}(\Phi)(x)
\end{aligned}
$$

wählen mit

$$
x_{i j}=-\cos \left(\frac{\pi(j-1)}{m_{i}-1}\right)
$$

und

$$
\mathbf{U}_{i, d}(\Phi)(x):=\sum_{\substack{1 \leq j_{l} \leq m_{i_{l}} \\ 1 \leq l \leq d}} \Phi\left(x_{i_{1} j_{1}}, \ldots, x_{i_{d} j_{d}}\right) x^{j}
$$

wobei $i=\left(i_{1}, \ldots, i_{d}\right)$ gilt. Unter diesen Voraussetzungen schreiben wir $\tilde{p}_{\Phi} \equiv A_{q, d}(\Phi)$ gemäß (9) aus Abschnitt 3 und folgern

$$
\begin{aligned}
E_{d}^{\mathcal{S}}(k, \phi, h) & \leq \max _{\|x\| \leq h}\left|I_{d}(\Phi)(x)-A_{q, d}(\Phi)(x)\right| \\
& \leq\left\|I_{d}(\Phi)-A_{q, d}(\Phi)\right\|_{[0, h]^{d}, \infty} \\
& \leq c d \max \left(2 c, c_{\nu}\right)^{d-1} 2^{-\nu k} k^{2 d-1}\|\Phi\|_{\mathcal{F} \otimes \ldots \otimes \mathcal{F}}
\end{aligned}
$$

Die letzte Ungleichung erhalten wir unter Nutzung von Satz 4.29.

Wir verdeutlichen nun die Zusammenhänge zwischen Fehlerabschätzung und Gitterpunktanzahl $n:=n(q, d)$ :

Korollar 5.15 Mit den Voraussetzungen von Satz 5.14 folgern wir für jede Funktion $f \in \mathcal{N}_{\Phi}$ und ihre Interpolante $s_{f, \Phi, X}$ auf dem gestörten dünnen Gitter $X=L_{q, d}^{C C}$ oder $L_{q, d}^{C C 2}$ mit $|X|=n$

$$
\left\|f-s_{f, \Phi, X}\right\|_{\infty} \leq C_{\nu, d} n^{-\frac{\nu}{2}}(2 d+\log (n))^{\frac{(\nu+2)(d-1)+1}{2}}\|f\|_{\Phi}
$$

mit

$$
C_{\nu, d}:=\left(1+c_{2}\right) \frac{c \sqrt{d} 2^{\frac{\nu}{2}}}{(d-1) !^{\frac{\nu}{2}}} \max \left(2 c^{2}, c_{\nu}\right)^{\frac{d-1}{2}}\|\Phi\|_{\mathcal{F} \otimes \ldots \otimes \mathcal{F}}^{\frac{1}{2}}
$$

Dabei gilt $c_{\nu}=1$ im Clenshaw-Curtis-Fall und $c_{\nu}=2^{\nu}$ falls $n=\left|L_{q, d}^{C C 2}\right|$. 


\subsubsection{Tensorprodukt-Basisfunktionen}

Naturgemäß kommt in diesem Zusammenhang solchen Basisfunktionen, welche sich als Tensorprodukt eindimensionaler Basisfunktionen schreiben lassen, besondere Bedeutung zu. In diesem Fall können die Ergebnisse von Lemma 5.11 auf weitere, verschiedenste Weisen angewandt werden. Wir erhalten damit zunächst folgenden

Satz 5.16 Für die natürlichen Zahlen $q, \tilde{q}, d, k, \tilde{m}_{1}, \ldots, \tilde{m}_{\tilde{q}-d+1}, m_{1}, \ldots, m_{q-d+1}$ gelte $\mathcal{P} \subset \mathcal{S P}_{\tilde{q}, d}^{\tilde{m}_{1}, \ldots, \tilde{m}_{\tilde{q}-d+1}}, \tilde{m}_{j} \leq m_{j}$ und $q \geq \tilde{q}, k \geq d$, wobei $\mathcal{P}$ einen d-variaten Polynomraum darstelle. Die bezüglich $\mathcal{P}$ bedingt positiv definite Funktion $\Phi$ sei eine d-variate Basisfunktion in Tensorproduktform; d.h. $\Phi=\phi_{1} \otimes \ldots \otimes \phi_{d}$ besteht aus dem Produkt von univariaten, um den Nullpunkt symmetrischen Funktionen $\phi_{j}, 1 \leq j \leq d$. Es gilt dann für alle Datensätze $X=\left\{x_{1}, \ldots, x_{N}\right\}$ mit $N=\left|H_{q, d}^{C C}\right|$ oder $\left|H_{q, d}^{C C 2}\right|$ und jeden Punkt $x \in \mathbb{R}^{d}$ mit

$$
h_{\zeta}(x)=\sup _{\|y-x\| \leq \zeta} \min _{z \in X}\|z-y\| \leq h_{0}=\frac{\zeta \epsilon_{0}}{m_{q, d}}
$$

und $\epsilon_{0}<\frac{1}{2}$ die Abschätzung

$$
P_{X, \tilde{u}, x}^{2} \leq\left(1+c_{2}\right)^{2} E_{d}^{\mathcal{S}, \otimes}(k, \Phi, \epsilon)
$$

mit $\epsilon \geq 2 c_{1} h_{\zeta}(x), c_{1}=1+\frac{m_{q, d}}{\epsilon_{0}}$, der von $\epsilon_{0}$ und $m_{q, d}=\max _{\substack{1 \leq j \leq d \\ i_{1}+\cdots+i_{d}=q}}\left(m_{i_{j}}-1\right)$ abhängigen Lebesgueschen Konstanten $c_{2} \in \mathbb{R}_{+}$, weiterhin

$$
\begin{aligned}
E_{d}^{\mathcal{S}, \otimes}(k, \Phi, \epsilon) & :=\prod_{\alpha=2}^{d}\left\|\phi_{\alpha}\right\|_{[0, \epsilon], \infty} E\left(m_{k-d+1}, \phi_{1}, \epsilon\right)+ \\
& +\sum_{\rho=1}^{d-1} \prod_{\alpha=0}^{\rho-2}\left\|\phi_{d-\alpha}\right\|_{[0, \epsilon], \infty} \sum_{|i|_{d-\rho} \leq k-\rho} \prod_{j=1}^{d-\rho}\left(E\left(m_{i_{j}}, \phi_{j}, \epsilon\right)+E\left(m_{i_{j}-1}, \phi_{j}, \epsilon\right)\right) . \\
& \cdot E\left(m_{k-\rho+1-|i|_{d-\rho}}, \phi_{d-\rho+1}, \epsilon\right),
\end{aligned}
$$

$E\left(0, \phi_{j}, \epsilon\right):=\left\|\phi_{j}\right\|_{[0, \epsilon], \infty}$, und

$$
E(l, \psi, \epsilon):=\inf _{p \in \mathbb{P}_{l}}\|\psi-p\|_{[0, \epsilon], \infty}
$$

für eine auf dem Intervall $[0, \epsilon]$ definierte univariate Funktion $\psi$ und für $1 \leq \beta \leq q-d+1$ 
Beweis: Es sei $p_{l}^{*, \psi} \in \mathbb{P}_{m_{l}}$ Lösung der Approximationsaufgabe (26), auch bezeichne für $i \in N^{d}$ das Polynom $p_{i}^{*}:=p_{i_{1}}^{*, \phi_{1}} \otimes \ldots \otimes p_{i_{d}}^{*, \phi_{d}}$, und es gelte

$$
p_{k, d}^{*}:=\sum_{|i|_{d} \leq k}(-1)^{k-|i|_{d}}\left(\begin{array}{c}
d-1 \\
k-|i|_{d}
\end{array}\right) p_{i}^{*} .
$$

Damit definieren wir für alle $y \in \mathbb{R}^{d}$

$$
Q_{x, \tilde{u}}(y):=p_{k, d}^{*}(x-y)-\sum_{j=1}^{N} \tilde{u}_{j}(x) p_{k, d}^{*}\left(x_{j}-y\right)
$$

Offenbar gilt $p_{k, d}^{*} \in \mathcal{S P}_{k, d}^{m_{1}, \ldots, m_{k-d+1}} \subset \mathcal{S P}_{q, d}^{m_{1}, \ldots, m_{q-d+1}}$ für $k \leq q$; deshalb verschwindet $Q_{x, \tilde{u}}(y)$ und wir folgern wie im Beweis von Satz 5.13

$$
p_{k, d}^{*}(0)-2 \sum_{j=1}^{N} \tilde{u}_{j}(x) p_{k, d}^{*}\left(x_{j}-x\right)+\sum_{i, j=1}^{N} \tilde{u}_{j}(x) p_{k, d}^{*}\left(x_{i}-x_{j}\right)=0 .
$$

Weiterhin stellen wir nun das Smolyak-Polynom $p_{k, d}^{*}$ mit Hilfe von Lemma 3.16 auf eine andere Art dar; es gilt

$$
\begin{aligned}
p_{k, d}^{*} & =\sum_{|i|_{d} \leq k}\left(p_{i_{1}}^{*, \phi_{1}}-p_{i_{1}-1}^{*, \phi_{1}}\right) \otimes \ldots \otimes\left(p_{i_{d}}^{*, \phi_{d}}-p_{i_{d}-1}^{*, \phi_{d}}\right) \\
& =\sum_{|i|_{d-1} \leq k-1}\left(p_{i_{1}}^{*, \phi_{1}}-p_{i_{1}-1}^{*, \phi_{1}}\right) \otimes \ldots \otimes\left(p_{i_{d-1}}^{*, \phi_{d-1}}-p_{i_{d-1}-1}^{*, \phi_{d-1}}\right) \otimes p_{q-|i|_{d-1}}^{*, \phi_{d}} .
\end{aligned}
$$

Wir schätzen nun in Analogie zur Methode von Wasilkowski und Woźniakowski [46] für alle $y=\left(y_{1}, \ldots, y_{d}\right) \in \mathbb{R}^{d}$ mit $\|y\| \leq \epsilon$ den Betrag der Differenz $\left|\Phi(y)-p_{k, d}^{*}(y)\right|$ ab. Es gilt mit $\tilde{y}=\left(y_{1}, \ldots, y_{d-1}\right)$

$$
\begin{aligned}
& \left|\Phi(y)-p_{k, d}^{*}(y)\right| \\
& \leq\left|\left(\left(\phi_{1} \otimes \ldots \otimes \phi_{d-1}-p_{k-1, d-1}^{*}\right) \otimes \phi_{d}\right)(y)\right|+ \\
& \quad+\left|\sum_{|i|_{d-1} \leq k-1} \prod_{j=1}^{d-1}\left(p_{i_{j}}^{*, \phi_{j}}-p_{i_{j}-1}^{*, \phi_{j}}\right)\left(y_{j}\right)\left(\phi_{d}-p_{k-|i|_{d-1}}^{*, \phi_{d}}\right)\left(y_{d}\right)\right| \\
& \leq\left\|\phi_{d}\right\|_{[0, \epsilon], \infty}\left|\left(\phi_{1} \otimes \ldots \otimes \phi_{d-1}-p_{k-1, d-1}^{*}\right)(\tilde{y})\right|+ \\
& \quad+\sum_{|i|_{d-1} \leq k-1} \prod_{j=1}^{d-1}\left|\left(p_{i_{j}}^{*, \phi_{j}}-p_{i_{j}-1}^{*, \phi_{j}}\right)\left(y_{j}\right)\right|\left|\phi_{d}\left(y_{d}\right)-p_{k-|i|_{d-1}}^{*, \phi_{d}}\left(y_{d}\right)\right|
\end{aligned}
$$




$$
\begin{aligned}
\leq & \left\|\phi_{d}\right\|_{[0, \epsilon], \infty}\left|\left(\phi_{1} \otimes \ldots \otimes \phi_{d-1}-p_{k-1, d-1}^{*}\right)(\tilde{y})\right|+ \\
& +\sum_{|i|_{d-1} \leq k-1} \prod_{j=1}^{d-1}\left(\left|\left(\phi_{j}-p_{i_{j}}^{*, \phi_{j}}\right)\left(y_{j}\right)\right|+\left|\left(\phi_{j}-p_{i_{j}-1}^{*, \phi_{j}}\right)\left(y_{j}\right)\right|\right)\left|\left(\phi_{d}-p_{k-|i|_{d-1}}^{*, \phi_{d}}\right)\left(y_{d}\right)\right| \\
\leq & \left\|\phi_{d}\right\|_{[0, \epsilon], \infty}\left|\left(\phi_{1} \otimes \ldots \otimes \phi_{d-1}-p_{k-1, d-1}^{*}\right)(\tilde{y})\right|+ \\
& +\sum_{|i|_{d-1} \leq k-1} \prod_{j=1}^{d-1}\left(E\left(m_{i_{j}}, \phi_{j}, \epsilon\right)+E\left(m_{i_{j}-1}, \phi_{j}, \epsilon\right)\right) E\left(m_{k-|i|_{d-1}}, \phi_{d}, \epsilon\right)
\end{aligned}
$$

mit $E\left(0, \phi_{j}, \epsilon\right)=\left\|\phi_{j}\right\|_{[0, \epsilon], \infty}$ und induktiv folgern wir für $1 \leq l \leq d-1$

$$
\begin{aligned}
& \left|\Phi(y)-p_{k, d}^{*}(y)\right| \\
& \leq \prod_{\alpha=0}^{l-1}\left\|\phi_{d-\alpha}\right\|_{[0, \epsilon], \infty}\left|\left(\phi_{1} \otimes \ldots \otimes \phi_{d-l}-p_{k-l, d-l}^{*}\right)(\tilde{y})\right|+ \\
& \quad+\sum_{\rho=1}^{l} \prod_{\alpha=0}^{\rho-2}\left\|\phi_{d-\alpha}\right\|_{[0, \epsilon], \infty} \sum_{|i|_{d-\rho} \leq k-\rho} \prod_{j=1}^{d-\rho}\left(E\left(m_{i_{j}}, \phi_{j}, \epsilon\right)+E\left(m_{i_{j}-1}, \phi_{j}, \epsilon\right)\right) . \\
& \quad \cdot E\left(m_{k-\rho+1-|i|_{d-\rho}}, \phi_{d-\rho+1}, \epsilon\right)
\end{aligned}
$$

mit $l=d-1$ folgt $\left|\Phi(y)-p_{k, d}^{*}(y)\right| \leq E_{d}^{\mathcal{S}, \otimes}(k, \Phi, \epsilon)$. Zudem gilt ebenfalls wie in Satz 5.13

$$
\begin{aligned}
\epsilon & \geq 2 c_{1} h_{\zeta}(x) \geq 2 \max _{\substack{1 \leq j \leq N \\
\tilde{u}_{j}(x) \neq 0}}\left\|x_{j}-x\right\| \geq\left\|x_{i}-x\right\|+\left\|x_{j}-x\right\| \\
& \geq\left|\left(x_{i}-x\right)^{T} e_{l}\right|+\left|\left(x_{j}-x\right)^{T} e_{l}\right|
\end{aligned}
$$

wobei mit $e_{l}, 1 \leq l \leq d$, der $l$-te Einheitsvektor gemeint ist, und ebenso $\epsilon \geq\left\|x_{j}-x\right\| \geq\left|\left(x_{j}-x\right)^{T} e_{l}\right|$ für alle $i, j$ mit $\tilde{u}_{i}(x), \tilde{u}_{j}(x) \neq 0$. Insgesamt folgern wir nun

$$
\begin{aligned}
P_{X, \tilde{u}, x}^{2}= & \left(\Phi(0)-p_{k, d}^{*}(0)\right)-\left(2 \sum_{j=1}^{N} \tilde{u}_{j}(x) \Phi\left(x_{i}-x\right)-2 \sum_{j=1}^{N} \tilde{u}_{j}(x) p_{k, d}^{*}\left(x_{i}-x\right)\right)+ \\
& +\left(\sum_{i, j=1}^{N} \tilde{u}_{i}(x) \tilde{u}_{j}(x) \Phi\left(x_{i}-x_{j}\right)-\sum_{i, j=1}^{N} \tilde{u}_{i}(x) \tilde{u}_{j}(x) p_{k, d}^{*}\left(x_{i}-x_{j}\right)\right) \\
\leq & \left(1+c_{2}\right)^{2} E_{d}^{\mathcal{S}, \otimes}(k, \Phi, \epsilon) .
\end{aligned}
$$


Bemerkung 5.17 Die Lebesgueschen Konstanten $c_{2}$ stimmen in beiden vorangehenden Sätzen 5.13 und 5.16 überein.

Bemerkung 5.18 Aufgrund von $\mathbb{P}_{m}^{d}=\mathcal{S P}_{m+d-1, d}^{1, \ldots, m}$ ist Satz 5.16 auch zur klassischen Fehlerabschätzung bei der Interpolation mit Tensorprodukt-Basisfunktionen verwendbar.

\subsubsection{Tensorprodukt-Basisfunktionen auf dem dünnen Gitter}

Im folgenden betrachten wir nun noch eine weitere Methode, den Interpolationsfehler von Tensorprodukt-Basisfunktionen abzuschätzen, welche allerdings nur auf einem dünnen Gitter funktioniert. Wir benutzen dazu das Störungslemma 2.8 nur im eindimensionalen, also klassischen, Fall. Auch hier resultieren im Vergleich zu den Sätzen 5.13 und 5.16 veränderte Lebesguesche Konstanten $c_{2}$ :

Satz 5.19 Die um den Nullpunkt symmetrischen univariaten Basisfunktionen $\phi_{j}$ mit $1 \leq j \leq d$ seien die Faktoren der d-variaten, bezüglich $\mathcal{P}$ bedingt positiv definiten Basisfunktion in Tensorproduktform $\Phi:=\phi_{1} \otimes \ldots \otimes \phi_{d}$. Für $q, \tilde{q}, d, k \in \mathbb{N}$, $\tilde{k}=\left(k_{1}, \ldots, k_{d}\right) \in \mathbb{N}^{d}$ mit $q \geq \tilde{q}, k, k_{\beta} \geq d$ und $\tilde{m}_{1}, \ldots, \tilde{m}_{\tilde{q}-d+1}, m_{1}, \ldots, m_{q-d+1} \in \mathbb{I}$ gelte $\mathcal{P} \subseteq \mathcal{S P}_{\tilde{q}, d}^{\tilde{m}_{1}, \ldots, \tilde{m}_{\tilde{q}-d+1}}$. Dann folgt für alle Datensätze auf einem dünnen Gitter $H_{q, d}=\bigcup_{|i|_{d}=q}\left(X_{i_{1}} \otimes \cdots \otimes X_{i_{d}}\right)$ mit $X_{j}=\left\{x_{j 1}, \ldots, x_{j m_{j}}\right\}$ und $x_{j l} \in \mathbb{R}$ für alle möglichen Werte von $j$ und $l$ und jeden Punkt $x=\left(x_{1}, \ldots, x_{d}\right) \in \mathbb{R}^{d}$ mit

$$
h_{\zeta}(x)=\sup _{\|y-x\| \leq \zeta} \min _{z \in H_{q, d}}\|z-y\| \leq h_{0}=\frac{\zeta \epsilon_{0}}{m_{q-d+1}-1},
$$

$\epsilon_{0}<\frac{1}{2}$ und mit $\epsilon \geq 2 c_{1} h_{\zeta}(x), c_{1}=\max _{1 \leq \beta \leq q-d+1}\left(1+\frac{m_{\beta}-1}{\epsilon_{0}}\right)$, der von $\epsilon_{0}$ und $m_{\beta}$, $1 \leq \beta \leq q-d+1$, abhängigen Lebesgueschen Konstanten $c_{2}^{*} \in \mathbb{R}_{+}$die Abschätzung

$$
P_{X, \tilde{u}, x}^{2} \leq\left(1+c_{2}^{*}\right)^{2} E_{d}^{\mathcal{S}, \otimes}(k, \Phi, \epsilon) .
$$

Beweis: Nach Satz 2.8 existieren zu allen $\beta \in \mathbb{N}, m_{\beta} \geq 1$ und zu allen $\zeta>0$ reelle Zahlen $h_{0, \beta}>0, c_{\beta, 1}, c_{\beta, 2}>0$, so daß es für alle $X_{\beta}=\left\{x_{\beta 1}, \ldots, x_{\beta m_{\beta}}\right\}, z \in \mathbb{R}$ und

$$
h_{\zeta, \beta}(z):=\sup _{\substack{|z-y| \leq \zeta \\ y \in \mathbb{R}}} \min _{1 \leq j \leq m_{\beta}}\left|x_{\beta j}-y\right| \leq h_{0, \beta}
$$


reelle $\tilde{u}_{\beta 1}(z), \ldots, \tilde{u}_{\beta m_{\beta}}(z)$ gibt mit

(1) $\sum_{j=1}^{m_{\beta}} \tilde{u}_{\beta j}(z) p\left(x_{\beta j}\right)=p(z)$ für alle $p \in \mathbb{P}_{m_{\beta}}$,

(2) $\tilde{u}_{\beta j}(z)=0$ für alle $j$ mit $\left|x_{\beta j}-z\right|>c_{\beta, 1} h_{\zeta}(z)$ wobei $c_{\beta, 1}=\left(1+\frac{m_{\beta}-1}{\epsilon_{0}}\right)$ und

(3) $\sum_{j=1}^{m_{\beta}}\left|\tilde{u}_{\beta j}(z)\right| \leq c_{\beta, 2}$.

Es existieren also zu vorgegebenen $\zeta, l, i_{l}, m_{i_{l}}$, zum dünnen Gitter $H_{q, d}$ und $x=\left(x_{1}, \ldots, x_{d}\right) \in \mathbb{R}^{d}$ reelle $\left\{\tilde{u}_{i_{l} j_{l}}\left(x_{l}\right)\right\}_{1 \leq j_{l} \leq m_{i_{l}}}$ mit

$$
\sum_{\substack{1 \leq j_{l} \leq m_{i_{l}} \\ 1 \leq l \leq d}}\left(\tilde{u}_{i_{1} j_{1}} \otimes \ldots \otimes \tilde{u}_{i_{d} j_{d}}\right)(x) p\left(x_{i_{1} j_{1}}, \ldots, x_{i_{d} j_{d}}\right)=p(x)
$$

für alle $p \in \mathbb{P}_{m_{i_{1}}} \otimes \ldots \otimes \mathbb{P}_{m_{i_{d}}}$; damit folgt unter Ausnutzung von Formel (A.4)

$$
\sum_{|i|_{d} \leq k}(-1)^{k-|i|_{d}}\left(\begin{array}{c}
d-1 \\
k-|i|_{d}
\end{array}\right) \sum_{\substack{1 \leq j_{l} \leq m_{i_{l}} \\
1 \leq l \leq d}} \tilde{u}_{i j}(x) p\left(x_{i j}\right)=p(x)
$$

$\operatorname{mit} i=\left(i_{1}, \ldots, i_{d}\right) \in \mathbb{N}^{d}, j=\left(j_{1}, \ldots, j_{d}\right) \in \mathbb{N}^{d}, \tilde{u}_{i, j}:=\tilde{u}_{i_{1}, j_{1}} \otimes \ldots \otimes \tilde{u}_{i_{d}, j_{d}}$ und $x_{i j}:=\left(x_{i_{1} j_{1}}, \ldots, x_{i_{d} j_{d}}\right)$ für alle $p \in \mathcal{S} \mathcal{P}_{k, d}^{m_{1}, \ldots, m_{k-d+1}}$. Weiterhin gelten die Eigenschaften (2) und (3) für alle möglichen Werte von $\beta$; deshalb folgt aus $\tilde{u}_{\beta j} \neq 0$ die Ungleichung $c_{1} h_{\zeta}(z) \geq\left|x_{\beta j}-z\right|$. Bezeichne nun $p_{l}^{*, j}$ die Lösung der Approximationsaufgabe

$$
\inf _{p \in \mathbb{P}_{l}}\left\|\phi_{j}-p\right\|_{[0, \epsilon], \infty}
$$

es gelte

$$
p_{k, d}^{*}:=\sum_{|i|_{d} \leq k}(-1)^{k-|i|_{d}}\left(\begin{array}{c}
d-1 \\
k-|i|_{d}
\end{array}\right)\left(p_{i_{1}}^{*, 1} \otimes \ldots \otimes p_{i_{d}}^{*, d}\right) .
$$

Mit $y \in \mathbb{R}^{d}$ definieren wir

$$
Q_{x, \tilde{u}}^{\delta}(y):=p_{k, d}^{*, \delta}(x-y)-\sum_{\substack{|i|_{d} \leq k \\ 1 \leq j_{l} \leq m_{i_{l}} \\ 1 \leq l \leq d}} \tilde{u}_{i j}(x) p_{k, d}^{*, \delta}\left(x_{i j}-y\right)=0
$$

für $k \leq q$ wegen $p_{k, d}^{*, \delta} \in \mathcal{S P}_{k, d}^{m_{1}, \ldots, m_{k-d+1}}$. Es gilt auch $\epsilon \geq 2 c_{1} h_{\zeta}(x)$, und unter der Bezeichnung $H_{q, d}=:\left\{x_{1}, \ldots, x_{N}\right\}$ mit $N=\left|H_{q, d}\right|$ folgern wir

$$
\epsilon \geq 2 c_{1} h_{\zeta}(x) \geq 2 c_{\beta, 1} h_{\zeta, \beta}\left(x^{T} e_{l}\right) \geq 2 \max _{\substack{1 \leq j \leq N \\ \tilde{u}_{l j} \neq 0}}\left|x^{T} e_{l}-x_{j}^{T} e_{l}\right|
$$




$$
\geq\left|x^{T} e_{l}-x_{i}^{T} e_{l}\right|+\left|x^{T} e_{l}-x_{j}^{T} e_{l}\right|
$$

mit für $1 \leq k \leq d, 1 \leq \beta \leq q-d+1$ und $1 \leq i, j \leq N$; dabei wird mit $e_{l}$ der $l$-te Einheitsvektor bezeichnet. Damit folgt analog zum Beweis von Satz 5.16 für alle $y \in \mathbb{R}^{d}$ mit $\|y\| \leq \epsilon$

$$
\left|\Phi(y)-p_{k, d}^{*, a}(y)\right| \leq E_{d}^{\mathcal{S}, \otimes}(k, \Phi, \epsilon)
$$

Daraus resultiert

$$
\begin{aligned}
P_{X, \tilde{u}, x}^{2}= & \left(\Phi(0)-p_{k, d}^{*, \delta}(0)\right)+ \\
& +2 \sum_{\substack{|i|_{d} \leq k \\
i \in N^{d} \\
1 \leq j_{l} \leq m_{i_{l}} \\
1 \leq l \leq d}} \tilde{u}_{i j}(x)\left(\Phi\left(x_{i j}-x\right)-p_{k, d}^{*, \delta}\left(x_{i j}-x\right)\right)+ \\
& +\sum_{\substack{|i|_{d} \leq k \\
1 \leq j_{l} \leq m_{i_{l}} \\
1 \leq l \leq d}} \sum_{\substack{|r|_{d} \leq k \\
1 \leq s_{l} \leq m_{r} l \\
1 \leq l \leq d}} \tilde{u}_{i j}(x) \tilde{u}_{r s}(x)\left(\Phi\left(x_{i j}-x_{r s}\right)-p_{k, d}^{*, \delta}\left(x_{i j}-x_{r s}\right)\right) .
\end{aligned}
$$

Wir folgern damit schließlich

$$
P_{X, \tilde{u}, x}^{2} \leq\left(1+c_{2}^{*}\right)^{2} E_{d}^{\mathcal{S}, \otimes}(k, \Phi, \epsilon)
$$

mit

$$
c_{2}^{*}:=\sum_{\substack{|i|_{d} \leq k \\ 1 \leq j_{l} \leq m_{i_{l}} \\ 1 \leq l \leq d}}\left|\tilde{u}_{i j}(x)\right|
$$

Bemerkung 5.20 Beim üblicherweise verwendeten sukzessive verschachtelten dünnen Gitter

$$
H_{q, d}=\bigcup_{|i|_{d}=q}\left(X_{i_{1}} \otimes \cdots \otimes X_{i_{d}}\right)
$$

mit $X_{1} \subset X_{2} \subset \ldots \subset X_{q-d+1}$ gilt natürlich $c_{1}=1+\frac{m_{q-d+1}-1}{\epsilon_{0}}$. Anders als bei Verwendung von Lemma 5.11 ist die Konstante $c_{1}$ hier in den meisten Fällen wesentlich kleiner. 


\subsubsection{Smolyak-Basisfunktionen}

Aufgrund ihrer besonderen Struktur bietet es sich an, Smolyak-BasisfunktionenInterpolanten näher zu untersuchen.

Satz 5.21 Es sei $\Phi$ Q-te Smolyakfunktion der Dimension d mit zugrundeliegenden univariaten Funktionen $\phi_{1}, \ldots, \phi_{Q-d+1} . \Phi$ sei bedingt positiv definit bezüglich des Polynomraumes $\mathcal{P} \subseteq \mathcal{S} \mathcal{P}_{\tilde{q}, d}^{\tilde{m}_{1}, \ldots, \tilde{m}_{\tilde{q}-d+1}}$. Dann gilt für jeden Datensatz $X=\left\{x_{1}, \ldots, x_{N}\right\}$ mit $N=\left|H_{Q, d}^{C C}\right|$ oder $\left|H_{Q, d}^{C C 2}\right|$ und jeden Punkt $x \in \mathbb{R}^{d}$ mit

$$
h_{\zeta}(x)=\sup _{\|x-y\| \leq \zeta} \min _{z \in X}\|z-y\| \leq h_{0}=\frac{\zeta \epsilon_{0}}{m_{q, d}}
$$

und $\epsilon_{0}<\frac{1}{2}$ die Abschätzung

$$
P_{X, \tilde{u}, x}^{2} \leq\left(1+c_{2}\right)^{2} E_{Q, d}^{\mathcal{S}, \Delta}(k, \Phi, \epsilon)
$$

mit $q \geq \tilde{q}, k \geq d, \epsilon \geq 2 c_{1} h_{\zeta}(x), c_{1}=1+\frac{m_{q, d}}{\epsilon_{0}}$, der von $\epsilon_{0}$ und $m_{Q, d}=\sum_{|i|_{d}=Q}\left(m_{i_{j}}-1\right)$ abhängigen Lebesgueschen Konstanten $c_{2} \in \mathbb{R}_{+}$,

$$
\begin{aligned}
& E_{Q, d}^{\mathcal{S}, \Delta}(k, \Phi, \epsilon):=M_{\Phi, \epsilon, Q-d+1}^{d-1} E\left(m_{k-d+1}, \phi_{Q-d+1}, \epsilon\right)+ \\
& \quad+\sum_{\alpha=1}^{d-1} M_{\Phi, \epsilon, Q-d+1}^{\alpha-1} \sum_{\substack{|l|_{d-\alpha} \leq Q-\alpha \\
|i|_{d-\alpha} \leq k-\alpha}} \prod_{\zeta=1}^{d-\alpha}\left(E\left(m_{i_{\zeta}}, \phi_{l_{\zeta}}, \epsilon\right)+E\left(m_{i_{\zeta}}, \phi_{l_{\zeta}-1}, \epsilon\right)+E\left(m_{i_{\zeta}-1}, \phi_{l_{\zeta}}, \epsilon\right)+\right. \\
& \left.\quad+E\left(m_{i_{\zeta}-1}, \phi_{l_{\zeta}-1}, \epsilon\right)\right) E\left(m_{k-\alpha+1-|i|_{d-\alpha}}, \phi_{Q-\alpha+1-|l|_{d-\alpha}}, \epsilon\right), \\
& E\left(m_{0}, \phi_{j}, \epsilon\right):=\left\|\phi_{j}\right\|_{[0, \epsilon], \infty} \text { und } M_{\Phi, \epsilon, J}:=\max _{1 \leq j \leq J}\left\|\phi_{j}\right\|_{[0, \epsilon], \infty} .
\end{aligned}
$$

Beweis: Wir gehen analog zum Beweis von Satz 5.16 vor; $p_{l}^{*, \psi}$ sei wie dort definiert. Wir schreiben $p_{j}^{*, \Delta, \phi_{l}}:=p_{j}^{*, \phi_{l}}-p_{j}^{*, \phi_{l-1}}$ für $1 \leq j \leq k-d+1$ und $1 \leq l \leq Q-d+1$. Weiterhin sei für $l \in \mathbb{N}^{d}$

$$
p_{k, d, l}^{*}:=\sum_{\substack{|i|_{d} \leq k \\ i \in \mathbb{N}^{d}}}\left(p_{i_{1}}^{*, \Delta, \phi_{l_{1}}}-p_{i_{1}-1}^{*, \Delta, \phi_{l_{1}}}\right) \otimes \ldots \otimes\left(p_{i_{d}}^{*, \Delta, \phi_{l}}-p_{i_{d}-1}^{*, \Delta, \phi_{l_{d}}}\right)
$$

und

$$
\tilde{p}_{k, d, Q}^{*}:=\sum_{\substack{|l|_{d} \leq Q \\ l \in N^{d}}} p_{k, d, l}^{*} \in \mathcal{S P}_{k, d}^{m_{1}, \ldots, m_{k-d+1}} \subset \mathcal{S} \mathcal{P}_{q, d}^{m_{1}, \ldots, m_{q-d+1}}
$$


Es gilt weiterhin wie bei $(27)$

$$
\begin{aligned}
\tilde{p}_{k, d, Q}^{*} & =\sum_{\substack{|l|_{d} \leq Q \\
|i|_{d-1} \leq k-1}}\left(p_{i_{1}}^{*, \Delta, \phi_{l_{1}}}-p_{i_{1}-1}^{*, \Delta, \phi_{l_{1}}}\right) \otimes \ldots \otimes\left(p_{i_{d-1}}^{*, \Delta, \phi_{l_{d}}}-p_{i_{d-1}-1}^{*, \Delta, \phi_{l_{d}}}\right) \otimes p_{k-|i|_{d-1}}^{*, \Delta, \phi_{l_{d}}} \\
& =\sum_{\substack{|l|_{d} \leq Q \\
|i|_{d-1} \leq k-1}}\left(p_{i_{1}}^{*, \Delta, \phi_{l_{1}}}-p_{i_{1}-1}^{*, \Delta, \phi_{l_{1}}}\right) \otimes \ldots \otimes\left(p_{i_{d-1}}^{*, \Delta, \phi_{l_{d}}}-p_{i_{d-1}-1}^{*, \Delta, \phi_{l_{d}}}\right) \otimes\left(p_{k-|i|_{d-1}}^{*, \phi_{l_{d}}}-p_{k-|i|_{d-1}}^{*, \phi_{l_{d}-1}}\right) \\
& =\sum_{\substack{|l|_{d-1} \leq Q-1 \\
|i|_{d-1} \leq k-1}}\left(p_{i_{1}}^{*, \Delta, \phi_{l_{1}}}-p_{i_{1}-1}^{*, \Delta, \phi_{l_{1}}}\right) \otimes \ldots \otimes\left(p_{i_{d-1}}^{*, \Delta, \phi_{l}}-p_{i_{d-1}-1}^{*, \Delta, \phi_{l_{d}}}\right) \otimes p_{k-|i|_{d-1}}^{*, \phi_{Q-|l|}} ;
\end{aligned}
$$

damit folgt für $y=\left(\tilde{y}, y_{d}\right) \in \mathbb{R}^{d}$ mit $\Delta_{1}:=\phi_{1}$ und $\Delta_{j}:=\phi_{j}-\phi_{j-1}, 2 \leq j \leq Q-d+1$

$$
\begin{aligned}
& \left|\sum_{|l|_{d} \leq Q}\left(\Delta_{l_{1}} \otimes \ldots \otimes \Delta_{l_{d}}-\tilde{p}_{k, d, l}^{*}\right)(y)\right| \\
& =\mid \sum_{|l|_{d-1} \leq Q-1}\left(\Delta_{l_{1}} \otimes \ldots \otimes \Delta_{l_{d-1}} \otimes \phi_{Q-|l|_{d-1}}\right)(y)- \\
& -\sum_{\substack{|l|_{d-1} \leq Q-1 \\
|i|_{d-1} \leq k-1}}\left(p_{i_{1}}^{*, \Delta, \phi_{l_{1}}}-p_{i_{1}-1}^{*, \Delta, \phi_{l_{1}}}\right) \otimes \ldots \otimes\left(p_{i_{d-1}}^{*, \Delta, \phi_{l_{d-1}}}-p_{i_{d-1}-1}^{*, \Delta, \phi_{l_{d-1}}}\right) \otimes p_{k-|i|_{d-1}}^{*,\left.\phi_{Q-|l|}\right|_{d-1}}(y) \mid \\
& \leq \mid \sum_{|l|_{d-1} \leq Q-1}\left(\Delta_{l_{1}} \otimes \ldots \otimes \Delta_{l_{d-1}} \otimes \phi_{Q-|l|_{d-1}}\right)(y)- \\
& -\sum_{\substack{|l|_{d-1} \leq Q-1 \\
|i|_{d-1} \leq k-1}}\left(p_{i_{1}}^{*, \Delta, \phi_{l_{1}}}-p_{i_{1}-1}^{*, \Delta, \phi_{l_{1}}}\right) \otimes \ldots \otimes\left(p_{i_{d-1}}^{*, \Delta, \phi_{l_{d}}}-p_{i_{d-1}-1}^{*, \Delta, \phi_{l_{d-1}}}\right) \otimes \phi_{Q-|l|_{d-1}}(y) \mid \\
& +\mid \sum_{\substack{\left.\left|l l_{d-1} \leq Q-1\\
\right| i\right|_{d-1} \leq k-1}}\left(p_{i_{1}}^{*, \Delta, \phi_{l_{1}}}-p_{i_{1}-1}^{*, \Delta, \phi_{l_{1}}}\right) \otimes \ldots \otimes\left(p_{i_{d-1}, \Delta, \phi_{l_{d-1}}}^{*}-p_{i_{d-1}-1}^{*, \Delta, \phi_{l}}\right) \otimes \\
& \otimes\left(p_{k-|i|_{d-1}^{*}}^{*, \phi_{Q-|l|_{d-1}}}-\phi_{Q-|l|_{d-1}}\right)(y) \mid \\
& \leq M_{\Phi, \epsilon, Q-d+1}\left|\sum_{|l|_{d-1} \leq Q-1}\left(\Delta_{l_{1}} \otimes \ldots \otimes \Delta_{l_{d-1}}-\tilde{p}_{k-1, d-1, l}^{*}\right)(\tilde{y})\right|+ \\
& +\sum_{\substack{|l|_{d-1} \leq Q-\left.1 \\
||_{d}\right|_{d-1} \leq k-1}} \prod_{\zeta=1}^{d-1}\left(E\left(m_{i_{\zeta}}, \phi_{l_{\zeta}}, \epsilon\right)+E\left(m_{i_{\zeta}}, \phi_{l_{\zeta}-1}, \epsilon\right)+E\left(m_{i_{\zeta}-1}, \phi_{l_{\zeta}}, \epsilon\right)+E\left(m_{i_{\zeta}-1}, \phi_{l_{\zeta}-1}, \epsilon\right)\right) . \\
& \text { - } E\left(m_{k-1-|i|_{d-1}}, \phi_{Q-1-|l|_{d-1}}, \epsilon\right) \text {; }
\end{aligned}
$$


induktiv folgt mit

$\left|\sum_{l_{1}=1}^{Q-d+1}\left(\Delta_{l_{1}}-\tilde{p}_{k-d+1,1, l_{1}}^{*}\right)\left(y_{1}\right)\right|=\left|\left(\phi_{Q-d+1}-p_{k-d+1}^{*, \phi_{Q-d+1}}\right)\left(y_{1}\right)\right| \leq E\left(k-d+1, \phi_{Q-d+1}, \epsilon\right)$

für $\left\|y_{1}\right\| \leq \epsilon$ analog zum Beweis von Satz 5.16 die Behauptung.

Im Fall $Q=k$ läßt sich eine weitere, einfachere obere Schranke für das Quadrat der Powerfunktion finden:

Satz 5.22 Unter den Voraussetzungen von Satz 5.21 gilt

$$
P_{X, \tilde{u}, x}^{2} \leq\left(1+c_{2}\right)^{2} \tilde{E}_{Q, d}^{\mathcal{S}, \Delta}(Q, \Phi, \epsilon)
$$

mit

$$
\begin{aligned}
\tilde{E}_{d}^{\mathcal{S}, \Delta}(Q, \Phi, \epsilon):= & M_{\Phi, \epsilon, Q-d+1}^{d-1} E\left(m_{Q-d+1}, \phi_{Q-d+1}, \epsilon\right)+ \\
& +\sum_{\alpha=1}^{d-1} M_{\Phi, \epsilon}^{\alpha-1} \sum_{\mid l_{d-\alpha} \leq Q-\alpha} \prod_{\zeta=1}^{d-\alpha}\left(E\left(m_{l_{\zeta}}, \phi_{l_{\zeta}}, \epsilon\right)+E\left(m_{l_{\zeta}}, \phi_{l_{\zeta}-1}, \epsilon\right)\right) . \\
& \cdot E\left(m_{Q-\alpha+1-|| l_{d-\alpha}},\left.\phi_{Q-\alpha+1-|l|}\right|_{d-\alpha}, \epsilon\right) .
\end{aligned}
$$

Beweis: Wir wählen $p_{j}^{*, \Delta, \phi_{l}}$ wie im Beweis von Satz 5.21 und definieren

$$
\tilde{p}_{Q, d}^{*}:=\sum_{\substack{\mid l l_{d} \leq Q \\ l \in \mathbb{N}^{d}}} p_{l_{1}}^{*, \Delta, \phi_{l_{1}}} \otimes \ldots \otimes p_{l_{d}}^{*, \Delta, \phi_{l_{d}}} .
$$

Wir folgern daraus

$$
\begin{aligned}
& \left|\sum_{|l|_{d} \leq Q}\left(\Delta_{l_{1}} \otimes \ldots \otimes \Delta_{l_{d}}\right)-\tilde{p}_{Q, d}^{*}\right|=\mid \sum_{\mid l l_{d-1} \leq Q-1}\left(\Delta_{l_{1}} \otimes \ldots \otimes \Delta_{l_{d-1}} \otimes \phi_{Q-\mid l l_{d-1}}\right)- \\
& \quad-\sum_{|l|_{d-1} \leq Q-1}\left(p^{*, \Delta, \phi_{l_{1}}} \otimes \ldots \otimes p^{\left.*, \Delta, \phi_{l_{d-1}} \otimes p_{Q-\mid l l_{d-1}}^{\phi_{Q-1}}\right) \mid}\right. \\
& \leq M_{\Phi, \epsilon}\left|\sum_{\mid l l_{d-1} \leq Q-1}\left(\Delta_{l_{1}} \otimes \ldots \otimes \Delta_{l_{d-1}}-p^{*, \Delta, \phi_{l_{1}}} \otimes \ldots \otimes p^{*, \Delta, \phi_{l_{d-1}}}\right)\right|+ \\
& \quad+\left|\sum_{\mid l l_{d-1} \leq Q-1} \prod_{\zeta=1}^{d-1}\left(E\left(m_{l_{\zeta}}, \phi_{l_{\zeta}}, \epsilon\right)+E\left(m_{l_{\zeta}}, \phi_{l_{\zeta}-1}, \epsilon\right)\right) E\left(m_{Q-|l|_{d-1}}, \phi_{Q-\mid l l_{d-1}}, \epsilon\right)\right| \\
& \leq \tilde{E}_{Q, d}^{\mathcal{S}, \Delta}(Q, \Phi, \epsilon)
\end{aligned}
$$


analog zum Beweis von Satz 5.21.

Bemerkung 5.23 Aufgrund von Lemma 3.37 gilt

$$
A_{\tilde{Q}, d}\left(\phi_{1}, \ldots, \phi_{\tilde{Q}-d+1}\right)=A_{Q, d}\left(\phi_{1}, \ldots, \phi_{1}, \phi_{2}, \ldots, \phi_{\tilde{Q}-d+1}\right)
$$

damit ist Satz 5.22 auch für $\tilde{Q}$-te Smolyakfunktionen mit $\tilde{Q} \leq Q$ anwendbar.

\subsubsection{Praktische Fehlerschranken}

Wir wollen nun die vorangegangenen Ergebnisse auf bestimmte Typen von Fehlern, welche bei der Bestapproximation univariater Funktionen entstehen, anwenden. Bisher hatten wir aus praktischen Gründen die Norm der univariaten Funktionen $\left\|\phi_{j}\right\|_{[0, h], \infty}$ auch mit $E\left(m_{0}, \phi_{j}, h\right)$ bzw. $E\left(0, \phi_{j}, h\right)$ bezeichnet. Diese Schreibweise müssen wir nun zugunsten der besseren Anwendbarkeit aufgeben und wir formulieren deshalb:

Satz 5.24 Unter den Voraussetzungen von Satz 5.16 oder Satz 5.19 gilt

$$
\begin{aligned}
& E_{d}^{\mathcal{S}, \otimes}(k, \Phi, h) \\
& =\sum_{\rho=0}^{d-1} \frac{1}{\rho !} \prod_{\alpha=\rho+2}^{d}\left\|\phi_{\alpha}\right\|_{[0, h], \infty}\left(\sum_{u=0}^{\rho-1}\left(\begin{array}{l}
\rho \\
u
\end{array}\right) \sum_{l=\rho-u}^{k-d} E\left(m_{k-d+1-l}, \phi_{\rho+1}, h\right) \sum_{\sigma \in S_{\rho}} .\right. \\
& \cdot \sum_{j=0}^{u}\left(\begin{array}{l}
u \\
j
\end{array}\right) \prod_{r=1}^{j}\left\|\phi_{\sigma(r)}\right\|_{[0, h], \infty} \prod_{p=j+1}^{u} E\left(m_{1}, \phi_{\sigma(p)}, h\right) \cdot \\
& \cdot \sum_{\substack{\sum_{\begin{subarray}{c}{\beta \\
i_{\sigma(j)} \in I^{\prime}} }}^{\rho} i_{\sigma(\beta)}=l} \\
{\prod_{j=u(j)}}\end{subarray}}^{\rho}\left(E\left(m_{i_{\sigma(\tilde{j})}+1}, \phi_{\sigma(\tilde{j})}, h\right)+E\left(m_{i_{\sigma(\tilde{j})}}, \phi_{\sigma(\tilde{j})}, h\right)\right)+ \\
& \left.+E\left(m_{k-d+1}, \phi_{\rho+1}, h\right) \sum_{\sigma \in S_{\rho}} \sum_{j=0}^{\rho}\left(\begin{array}{l}
\rho \\
j
\end{array}\right) \prod_{r=1}^{j}\left\|\phi_{\sigma(r)}\right\|_{[0, h], \infty} \prod_{p=j+1}^{\rho} E\left(m_{1}, \phi_{\sigma(p)}, h\right)\right) .
\end{aligned}
$$

Beweis: Sei $S_{k}$ die Gruppe der Permutationen der natürlichen Zahlen $\{1, \ldots, k\}$. Wir betrachten

$\sum_{|i|_{\rho} \leq k-d+\rho} \prod_{j=1}^{\rho}\left(E\left(m_{i_{j}}, \phi_{j}, h\right)+E\left(m_{i_{j}-1}, \phi_{j}, h\right)\right) E\left(m_{k-d+\rho+1-|i|_{\rho}}, \phi_{\rho+1}, h\right)$ 


$$
\begin{aligned}
& =\sum_{l=\rho}^{k-d+\rho} \sum_{|i|_{\rho}=l} \prod_{j=1}^{\rho}\left(E\left(m_{i_{j}}, \phi_{j}, h\right)+E\left(m_{i_{j}-1}, \phi_{j}, h\right)\right) E\left(m_{\left.k-d+\rho+1-l, \phi_{\rho+1}, h\right)}\right. \\
& =\sum_{l=2 \rho}^{k-d+\rho} \sum_{\substack{|i| \rho=l \\
i_{j}>1 \\
1 \leq j \leq \rho}} \prod_{j=1}^{\rho}\left(E\left(m_{i_{j}}, \phi_{j}, h\right)+E\left(m_{i_{j}-1}, \phi_{j}, h\right)\right) E\left(m_{k-d+\rho+1-l}, \phi_{\rho+1}, h\right)+
\end{aligned}
$$

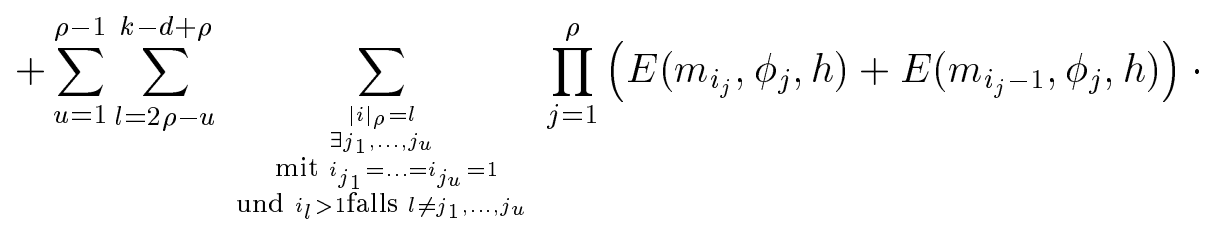

$$
\begin{aligned}
& \cdot E\left(m_{k-d+\rho+1-l}, \phi_{\rho+1}, h\right)+\prod_{j=1}^{\rho}\left(E\left(m_{1}, \phi_{j}, h\right)+E\left(m_{0}, \phi_{j}, h\right)\right) E\left(m_{k-d+1}, \phi_{\rho+1}, h\right) \\
& =\sum_{l=2 \rho}^{k-d+\rho} \sum_{\mid i_{\rho}=l-\rho} \prod_{j=1}^{\rho}\left(E\left(m_{i_{j}+1}, \phi_{j}, h\right)+E\left(m_{i_{j}}, \phi_{j}, h\right)\right) E\left(m_{\left.k-d+\rho+1-l, \phi_{\rho+1}, h\right)}+\right.
\end{aligned}
$$

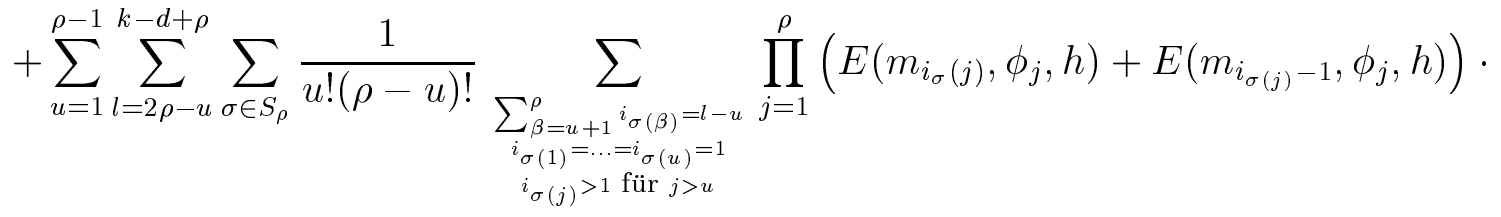

$$
\begin{aligned}
& \cdot E\left(m_{k-d+\rho+1-l}, \phi_{\rho+1}, h\right)+ \\
& +\prod_{j=1}^{\rho}\left(E\left(m_{1}, \phi_{j}, h\right)+E\left(m_{0}, \phi_{j}, h\right)\right) E\left(m_{k-d+1}, \phi_{\rho+1}, h\right) \\
& =\sum_{l=2 \rho}^{k-d+\rho} \sum_{|i|_{\rho}=l-\rho} \prod_{j=1}^{\rho}\left(E\left(m_{i_{j}}, \phi_{j}, h\right)+E\left(m_{i_{j}-1}, \phi_{j}, h\right)\right) E\left(m_{k-d+\rho+1-l}, \phi_{\rho+1}, h\right)+
\end{aligned}
$$

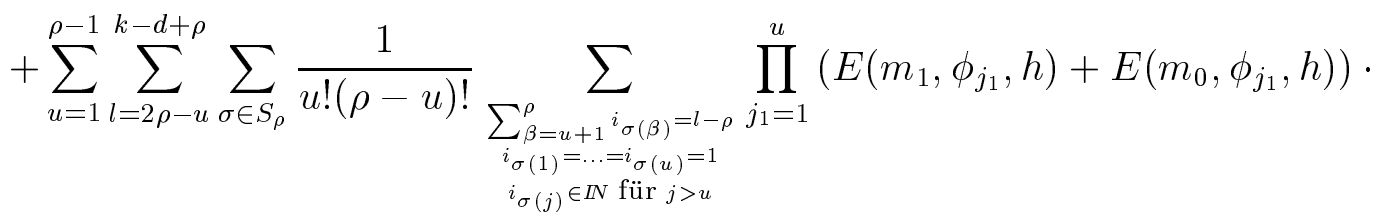

$$
\begin{aligned}
& \text {. } \prod_{j_{2}=u+1}^{\rho}\left(E\left(m_{i_{\sigma\left(j_{2}\right)}+1}, \phi_{\sigma\left(j_{2}\right)}, h\right)+E\left(m_{i_{\sigma\left(j_{2}\right)}}, \phi_{\sigma\left(j_{2}\right)}, h\right)\right) E\left(m_{k-d+\rho+1-l}, \phi_{\rho+1}, h\right)+ \\
& +\sum_{\sigma \in S_{\rho}} \frac{1}{\rho !} \prod_{j=1}^{\rho}\left(E\left(m_{1}, \phi_{j}, h\right)+E\left(m_{0}, \phi_{j}, h\right)\right) E\left(m_{k-d+1}, \phi_{\rho+1}, h\right)
\end{aligned}
$$

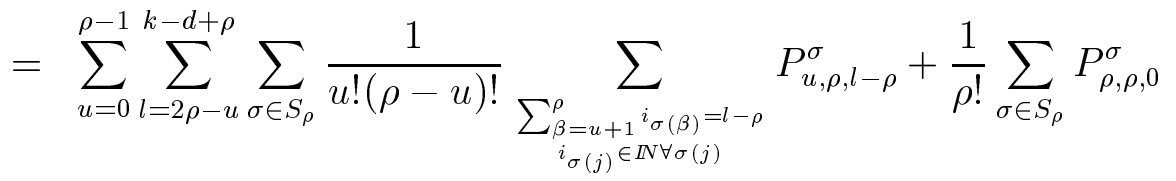




$$
=\sum_{u=0}^{\rho-1} \sum_{l=\rho-u}^{k-d} \sum_{\sigma \in S_{\rho}} \frac{1}{u !(\rho-u) !} \sum_{\substack{\sum_{\beta=u+1}^{\rho} i_{\sigma(\beta)}=l \\ i_{\sigma(j)} \in \mathbb{N} \forall \sigma(j)}} P_{u, \rho, l}^{\sigma}+\frac{1}{\rho !} \sum_{\sigma \in S_{\rho}} P_{\rho, \rho, 0}^{\sigma}
$$

mit

$$
\begin{aligned}
P_{u, \rho, l}^{\sigma}:= & \prod_{j_{1}=1}^{u}\left(E\left(m_{1}, \phi_{j_{1}}, h\right)+E\left(m_{0}, \phi_{j_{1}}, h\right)\right) \cdot \\
& \cdot \prod_{j_{2}=u+1}^{\rho}\left(E\left(m_{i_{\sigma\left(j_{2}\right)}+1}, \phi_{\sigma\left(j_{2}\right)}, h\right)+E\left(m_{i_{\sigma\left(j_{2}\right)}}, \phi_{\sigma\left(j_{2}\right)}, h\right)\right) E\left(m_{k-d+1-l}, \phi_{\rho+1}, h\right)
\end{aligned}
$$

für alle in Frage kommenden $u$ und $\rho$. Wir wenden uns nun $P_{u, \rho, l}^{\sigma}$ zu. Zunächst gilt für das Produkt von verschiedenen Summanden $a_{j}, b_{j}$

$$
\prod_{j=1}^{n}\left(a_{j}+b_{j}\right)=\sum_{j=0}^{n} \frac{1}{j !(n-j) !} \sum_{\sigma \in S_{n}} \prod_{p=1}^{j} a_{\sigma(p)} \prod_{r=j+1}^{n} b_{\sigma(r)},
$$

was man leicht induktiv beweisen kann. Wir wenden dieses Ergebnis nur auf das erste Produkt von $P_{u, \rho, l}^{\sigma}$ an, da eine Umformulierung des zweiten zwar möglich ist, aber keine praktische Relevanz besitzt. Die Menge der Permutationen auf $\{\sigma(j)\}_{1 \leq j \leq b}$ für $\sigma \in S_{n}, 1 \leq b \leq n$ nennen wir nun $S_{b}^{\sigma}$ und wir folgern damit

$$
\begin{aligned}
P_{u, \rho, l}^{\sigma}= & \sum_{j=0}^{u} \frac{1}{j !(u-j) !} \sum_{\sigma^{*} \in S_{u}^{\sigma}} \prod_{p=1}^{j}\left\|\phi_{\sigma^{*} \circ \sigma(p)}\right\|_{[0, h], \infty} \prod_{r=j+1}^{u} E\left(m_{1}, \phi_{\sigma^{*} \circ \sigma(r)}, h\right) \cdot \\
& \cdot \prod_{\tilde{j}=u+1}^{\rho}\left(E\left(m_{i_{\sigma(\tilde{j})}+1}, \phi_{\sigma(\tilde{j})}, h\right)+E\left(m_{i_{\sigma(\tilde{j})}}, \phi_{\sigma(\tilde{j})}, h\right)\right) E\left(m_{k-d+1-l}, \phi_{\rho+1}, h\right)
\end{aligned}
$$

und

$$
\begin{aligned}
\sum_{\sigma \in S_{\rho}} P_{u, \rho, l}^{\sigma}= & u ! \sum_{\sigma \in S_{\rho}} \sum_{j=0}^{u} \frac{1}{j !(u-j) !} \prod_{p=1}^{j}\left\|\phi_{\sigma(p)}\right\|_{[0, h], \infty} \prod_{r=j+1}^{u} E\left(m_{1}, \phi_{\sigma(r)}, h\right) \cdot \\
& \cdot \prod_{\tilde{j}=u+1}^{\rho}\left(E\left(m_{i_{\sigma(\tilde{j})}+1}, \phi_{\sigma(\tilde{j})}, h\right)+E\left(m_{i_{\sigma(\tilde{j})}}, \phi_{\sigma(\tilde{j})}, h\right)\right) E\left(m_{k-d+1-l}, \phi_{\rho+1}, h\right) .
\end{aligned}
$$

Die letzte Gleichung gilt deshalb, da jede Permutation

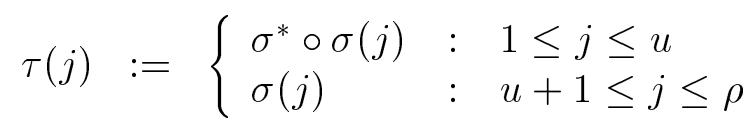


ein Element aus $S_{\rho}$ ist, welches auf $u$ ! möglichen Wegen entstehen kann.

Wir wollen nun dieses Ergebnis auf verschiedene Fälle anwenden. Wir starten mit den einfachsten Voraussetzungen:

Korollar 5.25 Es gelten die Voraussetzungen von Satz 5.16 oder Satz 5.19; weiterhin sei für alle $l \in \mathbb{N}$

$$
E\left(l, \phi_{\rho}, h\right) \leq K_{\phi_{\rho}}(h)
$$

Dann folgt für alle $m_{j}, 1 \leq j \leq q-d+1$

$$
E_{d}^{\mathcal{S}, \otimes}(k, \Phi, h) \leq \max _{0 \leq \rho \leq d-1}\left(2^{\rho} \tilde{K}_{\rho, d}(h)\right)\left(\begin{array}{c}
k \\
d-1
\end{array}\right)
$$

mit

$$
\tilde{K}_{\rho, d}(h):=\frac{1}{\rho !} \sum_{\sigma \in S_{\rho}} \max _{0 \leq j \leq \rho} \prod_{r=1}^{j}\left\|\phi_{\sigma(r)}\right\|_{[0, h], \infty} \prod_{p=j+1}^{\rho} K_{\phi_{\sigma(p)}}(h) K_{\phi_{\rho+1}}(h) \prod_{\alpha=\rho+2}^{d}\left\|\phi_{\alpha}\right\|_{[0, h], \infty} .
$$

Gilt $\phi_{1}=\ldots=\phi_{d}=: \phi$, so ist

$$
E_{d}^{\mathcal{S}, \otimes}(k, \Phi, h) \leq K_{\phi}(h)\left(\max \left(K_{\phi}(h),\|\phi\|_{[0, h], \infty}\right)+K_{\phi}(h)\right)^{d-1}\left(\begin{array}{c}
k \\
d-1
\end{array}\right) .
$$

Bemerkung 5.26 Da Korollar 5.25 für allgemeine $m_{j}$ Gültigkeit besitzt, kann es wegen $\mathbb{P}_{m}^{d}=\mathcal{S P}_{m+d-1, d}^{1, \ldots, m}$ zur klassischen Fehlerabschätzung bei der Interpolation mit Tensorprodukt-Basisfunktionen genutzt werden.

Beweis des Korollars: Es gilt mit

$$
\begin{aligned}
& \frac{1}{\rho !} \sum_{\sigma \in S_{\rho}} 2^{-u} \sum_{j=0}^{u}\left(\begin{array}{c}
u \\
j
\end{array}\right) \prod_{r=1}^{j}\left\|\phi_{\sigma(r)}\right\|_{[0, h], \infty} \prod_{p=j+1}^{\rho} K_{\phi_{\sigma(p)}}(h) K_{\phi_{\rho+1}}(h) \prod_{\alpha=\rho+2}^{d}\left\|\phi_{\alpha}\right\|_{[0, h], \infty} \\
& \quad \leq \frac{1}{\rho !} \sum_{\sigma \in S_{\rho}} \max _{1 \leq j \leq u} \prod_{r=1}^{j}\left\|\phi_{\sigma(r)}\right\|_{[0, h], \infty} \prod_{p=j+1}^{\rho} K_{\phi_{\sigma(p)}}(h) K_{\phi_{\rho+1}}(h) \prod_{\alpha=\rho+2}^{d}\left\|\phi_{\alpha}\right\|_{[0, h], \infty} \\
& \quad \leq \frac{1}{\rho !} \sum_{\sigma \in S_{\rho}} \max _{1 \leq j \leq \rho} \prod_{r=1}^{j}\left\|\phi_{\sigma(r)}\right\|_{[0, h], \infty} \prod_{p=j+1}^{\rho} K_{\phi_{\sigma(p)}}(h) K_{\phi_{\rho+1}}(h) \prod_{\alpha=\rho+2}^{d}\left\|\phi_{\alpha}\right\|_{[0, h], \infty} \\
& =: \tilde{K}_{\rho, d}(h)
\end{aligned}
$$


die Ungleichung

$$
\begin{aligned}
E_{q, d}^{\mathcal{S}, \otimes}(k, \Phi, h) & \leq \sum_{\rho=0}^{d-1} 2^{\rho}\left(\sum_{u=0}^{\rho-1}\left(\begin{array}{l}
\rho \\
u
\end{array}\right) \tilde{K}_{\rho, d}(h) \sum_{l=\rho-u}^{k-d}\left(\begin{array}{c}
l-1 \\
\rho-u-1
\end{array}\right)+\tilde{K}_{\rho, d}(h)\right) \\
& =\sum_{\rho=0}^{d-1} 2^{\rho} \tilde{K}_{\rho, d}(h)\left(\sum_{u=0}^{\rho-1}\left(\begin{array}{l}
\rho \\
u
\end{array}\right)\left(\begin{array}{c}
k-d \\
\rho-u
\end{array}\right)+1\right) \\
& =\sum_{\rho=0}^{d-1} 2^{\rho} \tilde{K}_{\rho, d}(h) \sum_{u=0}^{\rho}\left(\begin{array}{l}
\rho \\
u
\end{array}\right)\left(\begin{array}{c}
k-d \\
\rho-u
\end{array}\right) \\
& =\sum_{\rho=0}^{d-1} 2^{\rho} \tilde{K}_{\rho, d}(h)\left(\begin{array}{c}
k-d+\rho \\
\rho
\end{array}\right)
\end{aligned}
$$

wegen der Formeln (A.2) und (A.5) aus dem Anhang; es folgt die Behauptung. Bei Übereinstimmung aller univariater Funktionen $\phi_{j}$ gilt

$$
\begin{aligned}
\frac{1}{\rho !} \sum_{\sigma \in S_{\rho}} 2^{\rho-u} \sum_{j=0}^{u}\left(\begin{array}{l}
u \\
j
\end{array}\right) & \prod_{r=1}^{j}\left\|\phi_{\sigma(r)}\right\|_{[0, h], \infty} \prod_{p=j+1}^{\rho} K_{\phi_{\sigma(p)}}(h) K_{\phi_{\rho+1}}(h) \prod_{\alpha=\rho+2}^{d}\left\|\phi_{\alpha}\right\|_{[0, h], \infty} \\
& =2^{\rho}\|\phi\|_{[0, h], \infty}^{d-\rho-1} K_{\phi}(h)^{\rho-u+1}\left(\frac{\|\phi\|_{[0, h], \infty}+K_{\phi}(h)}{2}\right)^{u} \\
& =2^{\rho}\|\phi\|_{[0, h], \infty}^{d-\rho-1} K_{\phi}(h)^{\rho+1}\left(\frac{\|\phi\|_{[0, h], \infty}}{2 K_{\phi}(h)}+\frac{1}{2}\right)^{u} \\
& \leq 2^{\rho}\|\phi\|_{[0, h], \infty}^{d-\rho-1} K_{\phi}(h)^{\rho+1} \max _{0 \leq u \leq \rho}\left(\frac{\|\phi\|_{[0, h], \infty}}{2 K_{\phi}(h)}+\frac{1}{2}\right)^{u} \\
& =2^{\rho}\|\phi\|_{[0, h], \infty}^{d-\rho-1} K_{\phi}(h)^{\rho+1}\left(\frac{C_{\phi}(h)}{2 K_{\phi}(h)}+\frac{1}{2}\right)^{\rho} \\
& \leq K_{\phi}(h)_{0 \leq \rho \leq d-1}\|\phi\|_{[0, h], \infty}^{d-1-\rho}\left(C_{\phi}(h)+K_{\phi}(h)\right)^{\rho} \\
& =K_{\phi}(h) \max _{\left.0 . \phi \phi \|_{[0, h], \infty}, C_{\phi}(h)+K_{\phi}(h)\right)^{d-1}} \\
& =K_{\phi}(h)\left(C_{\phi}(h)+K_{\phi}(h)\right)^{d-1}
\end{aligned}
$$

$\operatorname{mit} C_{\phi}(h):=\max \left(K_{\phi}(h),\|\phi\|_{[0, h], \infty}\right)$. 
Wir bekommen eine andere Formulierung von Korollar 5.25, falls wir die Fehlerschranke in Abhängigkeit von der Gitterpunktanzahl $n:=n(q, d)$ des jeweiligen dünnen Gitters formulieren:

Korollar 5.27 Sei $n:=n(q, d)$ die Anzahl der Punkte des Clenshaw-Curtis-Gitters $H_{q, d}^{C C}$ bzw. $H_{q, d}^{C C 2}$. Unter den Voraussetzungen von Korollar 5.25 gilt

$$
E_{d}^{\mathcal{S}, \otimes}(k, \phi, h) \leq \frac{c_{\nu}^{d}}{(d-1) !} \max _{0 \leq \rho \leq d-1}\left(2^{\rho} \tilde{K}_{\rho, d}(h)\right)(2 d+\log (n))^{d-1}
$$

$b z w$.

$$
E_{d}^{\mathcal{S}, \otimes}(q, \phi, h) \leq \frac{K_{\phi}(h) c_{\nu}^{d}}{(d-1) !}\left(\max \left(K_{\phi}(h),\|\phi\|_{[0, h], \infty}\right)+K_{\phi}(h)\right)^{d-1}(2 d+\log (n))^{d-1}
$$

im Fall $\phi_{1}=\ldots=\phi_{d}=\phi$. Wir bezeichnen dabei $c_{\nu}=1$ im Clenshaw-Curtis-Fall und $c_{\nu}=2^{\nu}$ für $n=\left|H_{q, d}^{C C 2}\right|$. Ist $n:=n(q, d)$ die Anzahl der Punkte des dünnen Gitters $H_{q, d}$ mit $m_{j}=z \cdot j$ für $1 \leq j \leq q-d+1$, so folgern wir unter den Voraussetzungen von Korollar 5.25

$$
E_{d}^{\mathcal{S}, \otimes}(q, \phi, h) \leq \max _{0 \leq \rho \leq d-1}\left(2^{\rho} \tilde{K}_{\rho, d}(h)\right) \frac{n}{d z^{d}}
$$

und für $\phi_{1}=\ldots=\phi_{d}=\phi$ gilt

$$
E_{d}^{\mathcal{S}, \otimes}(q, \phi, h) \leq K_{\phi}(h)\left(\max \left(K_{\phi}(h),\|\phi\|_{[0, h], \infty}\right)+K_{\phi}(h)\right)^{d-1} \frac{n}{d z^{d}} .
$$

Offenbar kann mit univariaten Fehlerschranken wie in Korollar 5.25 der Fluch der Dimension nicht behoben werden. Deshalb gehen wir im folgenden zu besser anwendbaren eindimensionalen Schranken über. Das folgende Ergebnis stellt eine Verallgemeinerung von Korollar 5.25 dar:

Korollar 5.28 Die Voraussetzungen von Satz 5.16 oder Satz 5.19 seien erfüllt. Es gelte

$$
E\left(l, \phi_{j}, h\right) \leq K_{\phi_{j}}(h)(f(h))^{l}
$$

mit $l \in \mathbb{N}, 1 \leq l \leq q-d+1$. Dann folgern wir für $m_{1}=1,2, m_{j}=2^{j-1}+1$, $2 \leq j \leq q-d+1$ und $f(h) \leq 1$

$$
E_{d}^{\mathcal{S}, \otimes}(k, \Phi, h) \leq(f(h))^{2(k-d)} \max _{0 \leq \rho \leq d-1}\left(\frac{\left(1+f^{2}(h)\right)^{\rho}}{g(h)^{\rho+1}} \tilde{K}_{\rho, d}(h)\right)\left(\begin{array}{c}
k \\
d-1
\end{array}\right),
$$


wobei $\tilde{K}_{\rho, d}(h)$ wie in Korollar 5.25 definiert ist. Im Fall $\phi_{1}=\ldots=\phi_{d}=$ : $\phi$ gilt

$$
\begin{aligned}
& E_{d}^{\mathcal{S}, \otimes}(k, \Phi, h) \\
& \quad \leq \frac{(f(h))^{2(k-d)}}{g(h)} K_{\phi}(h)\left(\max \left(\frac{K_{\phi}(h)}{g(h)},\|\phi\|_{[0, h], \infty}\right)+\frac{f^{2}(h)}{g(h)} K_{\phi}(h)\right)^{d-1}\left(\begin{array}{c}
k \\
d-1
\end{array}\right)
\end{aligned}
$$

mit $g(h)=\left\{\begin{array}{lll}f(h) & : & X=L_{q, d}^{C C} \\ 1 & : & X=L_{q, d}^{C C 2}\end{array}\right.$.

Beweis: Wir führen den Beweis für $n=\left|H_{q, d}^{C C 2}\right|$ durch. Im Clenshaw-Curtis-Fall nutzen wir dieses Ergebnis, wobei wir die Konstanten $K_{\phi_{j}}, 1 \leq j \leq d$, durch $f(h)$ dividieren. Es gilt

$$
\begin{aligned}
& \prod_{\tilde{j}=u+1}^{\rho}\left(E\left(m_{i_{\sigma(\tilde{j})}+1}, \phi_{\sigma(\tilde{j})}, h\right)+E\left(m_{i_{\sigma(\tilde{j})}}, \phi_{\sigma(\tilde{j})}, h\right)\right) E\left(m_{k-d+1-l}, \phi_{\rho+1}, h\right) \\
& \leq\left(\prod_{\tilde{j}=u+1}^{\rho} K_{\phi_{\sigma(\tilde{j})}}(h)\right) K_{\phi_{\rho+1}}(h)(f(h))^{\sum_{w=u+1}^{\rho} 2^{i} \sigma(w)}\left(\frac{1+f^{2}(h)}{f(h)}\right)^{\rho-u}(f(h))^{2^{k-d-l}} \\
& \leq\left(\prod_{\tilde{j}=u+1}^{\rho} K_{\phi_{\sigma(\tilde{j})}}(h)\right) K_{\phi_{\rho+1}}(h)(f(h))^{2 \sum_{w=u+1}^{\rho} i_{\sigma(w)}}\left(1+f^{2}(h)\right)^{\rho-u}(f(h))^{2(k-d-l)} \\
& \quad=\left(\prod_{\tilde{j}=u+1}^{\rho} K_{\phi_{\sigma(\tilde{j})}}(h)\right) K_{\phi_{\rho+1}}(h)(f(h))^{2(k-d)}\left(1+f^{2}(h)\right)^{\rho-u} .
\end{aligned}
$$

Analog zum Beweis von Korollar 5.25 folgern wir

$$
\begin{aligned}
\left(1+f^{2}(h)\right)^{u} \tilde{K}_{\rho, d}(h) & \\
\geq & \frac{1}{\rho !} \sum_{\sigma \in S_{\rho}}(f(h))^{2 u} \sum_{j=0}^{u}\left(\begin{array}{l}
u \\
j
\end{array}\right)(f(h))^{-2 j} \prod_{r=1}^{j}\left\|\phi_{\sigma(r)}\right\|_{[0, h], \infty} \cdot \\
& \cdot \prod_{p=j+1}^{\rho} K_{\phi_{\sigma(p)}}(h) K_{\phi_{\rho+1}}(h) \prod_{\alpha=\rho+2}^{d}\left\|\phi_{\alpha}\right\|_{[0, h], \infty},
\end{aligned}
$$

damit gilt

$$
E_{d}^{\mathcal{S}, \otimes}(k, \Phi, h) \leq(f(h))^{2(k-d)} \sum_{\rho=0}^{d-1} \tilde{K}_{\rho, d}(h)\left(1+f^{2}(h)\right)^{\rho}\left(\sum_{u=0}^{\rho-1}\left(\begin{array}{l}
\rho \\
u
\end{array}\right) \sum_{l=\rho-u}^{k-d}\left(\begin{array}{c}
l-1 \\
\rho-u-1
\end{array}\right)+1\right)
$$


wie im Beweis von Korollar 5.25 erhalten wir das Behauptete. Falls $\phi_{j}=\phi$ für alle $j$ gilt mit $C_{\phi}(h):=\max \left(K_{\phi}(h),\|\phi\|_{[0, h], \infty}\right)$

$$
\begin{aligned}
& \left(1+f^{2}(h)\right)^{\rho-u} \frac{1}{\rho !} \sum_{\sigma \in S_{\rho}}(f(h))^{2 u} \sum_{j=0}^{u}\left(\begin{array}{l}
u \\
j
\end{array}\right)(f(h))^{-2 j} \prod_{r=1}^{j}\left\|\phi_{\sigma(r)}\right\|_{[0, h], \infty} \prod_{p=j+1}^{\rho} K_{\phi_{\sigma(p)}}(h) \cdot \\
& \cdot K_{\phi_{\rho+1}}(h) \prod_{\alpha=\rho+2}^{d}\left\|\phi_{\alpha}\right\|_{[0, h], \infty} \\
& =\left(1+f^{2}(h)\right)^{\rho-u}(f(h))^{2 u} \sum_{j=0}^{u}\left(\begin{array}{c}
u \\
j
\end{array}\right)(f(h))^{-2 j} K_{\phi}(h)^{\rho-j+1}\|\phi\|_{[0, h], \infty}^{d+j-\rho-1} \\
& =K_{\phi}(h)^{\rho+1}\left(1+f^{2}(h)\right)^{\rho}\|\phi\|_{[0, h], \infty}^{d-\rho-1}\left(\frac{f(h)^{2} K_{\phi}(h)+\|\phi\|_{[0, h], \infty}}{K_{\phi}(h)\left(1+f^{2}(h)\right)}\right)^{u} \\
& =K_{\phi}(h)^{\rho+1}\left(1+f^{2}(h)\right)^{\rho}\|\phi\|_{[0, h], \infty}^{d-\rho-1}\left(1+\frac{\|\phi\|_{[0, h], \infty}-K_{\phi}(h)}{K_{\phi}(h)\left(1+f^{2}(h)\right)}\right)^{u} \\
& \leq K_{\phi}(h)^{\rho+1}\left(1+f^{2}(h)\right)^{\rho}\|\phi\|_{[0, h], \infty}^{d-\rho-1}\left(1+\frac{C_{\phi}(h)-K_{\phi}(h)}{K_{\phi}(h)\left(1+f^{2}(h)\right)}\right)^{\rho} \\
& =K_{\phi}(h)\|\phi\|_{[0, h], \infty}^{d-1}\left(\frac{K_{\phi}(h)\left(1+f^{2}(h)\right)+C_{\phi}(h)-K_{\phi}(h)}{\|\phi\|_{[0, h], \infty}}\right)^{\rho} \\
& =K_{\phi}(h)\|\phi\|_{[0, h], \infty}^{d-1}\left(\frac{K_{\phi}(h) f^{2}(h)+C_{\phi}(h)}{\|\phi\|_{[0, h], \infty}^{\rho}}\right)^{d-1} \cdot \\
& \leq K_{\phi}(h)\left(K_{\phi}(h) f^{2}(h)+C_{\phi}(h)\right)^{d} \cdot
\end{aligned}
$$

Es folgt damit

Korollar 5.29 Unter den Voraussetzungen von Korollar 5.28 gilt für die Punktanzahl $n=n(q, d)$ des dünnen Gitters mit $m_{j}=2^{j-1}+1,1 \leq j \leq q-d+1$,

$$
\begin{aligned}
& E_{d}^{\mathcal{S}, \otimes}(q, \Phi, h) \\
& \quad \leq \frac{(2 d+\log (n))^{d}}{(d-1) !}(f(h))^{2(d-1) !-2(d-1)+n-2(d-1)(2 d+\log (n))} \max _{0 \leq \rho \leq d-1}\left(\left(1+f^{2}(h)\right)^{\rho} \tilde{K}_{\rho, d}(h)\right)
\end{aligned}
$$

bzw.

$$
\begin{aligned}
& E_{d}^{\mathcal{S}, \otimes}(q, \Phi, h) \\
& \quad \leq \frac{(2 d+\log (n))^{d}}{(d-1) !}(f(h))^{2(d-1) !+n-2(d-1)(2 d+\log (n))} \max _{0 \leq \rho \leq d-1}\left(\left(\frac{1+f^{2}(h)}{f(h)}\right)^{\rho} \tilde{K}_{\rho, d}(h)\right)
\end{aligned}
$$


im Clenshaw-Curtis-Fall.

Bemerkung 5.30 Möglich wird der Beweis von Korollar 5.28 durch die Abschätzung $f(h)^{2^{j}} \leq f(h)^{2 \cdot j}$; tatsächlich bekommen wir eine ähnliche Schranke im Fall $m_{j}=2 \cdot j$. Die Bestimmung schärferer Schranken ist schon im Fall $d=2$ relativ kompliziert. Ersetzen wir nun unter ansonsten gleichbleibenden Voraussetzungen die Bedingung $m_{j}=2^{j-1}+1$ durch $m_{j}=z \cdot j, z \in \mathbb{N}$, so erhalten wir bei fast analogem Beweis die Fehlerabschätzung

$$
E_{d}^{\mathcal{S}, \otimes}(k, \Phi, h) \leq(f(h))^{z(k-d+1)} \max _{0 \leq \rho \leq d-1}\left(\left(1+f^{z}(h)\right)^{\rho} \tilde{K}_{\rho, d}(h)\right)\left(\begin{array}{c}
k \\
d-1
\end{array}\right)
$$

für beliebige $\phi_{j}, 1 \leq j \leq d$, und

$$
\begin{aligned}
& E_{d}^{\mathcal{S}, \otimes}(k, \Phi, h) \\
& \quad \leq(f(h))^{z(k-d+1)} K_{\phi}(h)\left(\max \left(K_{\phi}(h),\|\phi\|_{[0, h], \infty}\right)+f^{z}(h) K_{\phi}(h)\right)^{d-1}\left(\begin{array}{c}
k \\
d-1
\end{array}\right)
\end{aligned}
$$

falls $\phi_{1}=\ldots=\phi_{d}=: \phi$. In Abhängigkeit von der Punktanzahl des entsprechenden dünnen Gitters $H_{q, d}$ mit $m_{j}=z \cdot j$ gilt aber $n:=n(q, d)=z^{d}\left(\begin{array}{l}q \\ d\end{array}\right)$ und wir folgern

$$
E_{d}^{\mathcal{S}, \otimes}(q, \Phi, h) \leq \frac{n}{d z^{d}}(f(h))^{\frac{n \frac{1}{d}}{d}}(f(h))^{-z(d-1)} \max _{0 \leq \rho \leq d-1}\left(\left(1+f^{z}(h)\right)^{\rho} \tilde{K}_{\rho, d}(h)\right)
$$

im allgemeinen Fall und

$$
\begin{aligned}
& E_{d}^{\mathcal{S}, \otimes}(q, \Phi, h) \\
& \quad \leq \frac{n}{d z^{d}}(f(h))^{\frac{n^{\frac{1}{d}}}{d}}(f(h))^{-z(d-1)} K_{\phi}(h)\left(\max \left(K_{\phi}(h),\|\phi\|_{[0, h], \infty}\right)+f^{z}(h) K_{\phi}(h)\right)^{d-1}
\end{aligned}
$$

für $\phi_{1}=\ldots=\phi_{d}=: \phi$.

Für $z=1$ und $q=m+d-1$ befinden wir uns im Fall der Interpolation mit Tensorprodukt-Basisfunktionen und dem klassischen Polynomraum $\mathbb{P}_{m}^{d}$, vgl. Bemerkung 5.18 .

Korollar 5.31 Unter den Voraussetzungen von Satz 5.16 oder Satz 5.19 gelte für $l \in I N$

$$
E\left(l, \phi_{j}, h\right) \leq K_{\phi_{j}}(h) l^{-\nu}
$$

mit $\nu \in \mathbb{R}$. Dann folgt

$$
E_{d}^{\mathcal{S}, \otimes}(k, \Phi, h) \leq 2^{-(k-d+1) \nu} \max _{0 \leq \rho \leq d-1}\left(M_{\rho, \nu}\left(\frac{2^{\nu}}{c_{\nu}}\right)^{\rho+1} \tilde{K}_{\rho, d}(h)\right)\left(\begin{array}{c}
k \\
d-1
\end{array}\right)
$$


mit

$$
M_{\rho, \nu}=\left\{\begin{array}{ll}
2^{\rho} & : \quad \nu \geq 0 \\
\left(1+2^{\nu}\right)^{\rho} & : \quad \nu<0
\end{array} .\right.
$$

Weiterhin gilt

$$
E_{d}^{\mathcal{S}, \otimes}(k, \Phi, h) \leq 2^{-(k-d+1) \nu}\left(\frac{2^{\nu}}{c_{\nu}}\right)^{d} K_{\phi}(h)\left(K_{\phi}(h)+2^{\nu} C_{\phi, \nu}\right)^{d-1}\left(\begin{array}{c}
k \\
d-1
\end{array}\right)
$$

falls $\phi_{1}=\ldots=\phi_{d}=: \phi$ und $C_{\phi, \nu}:=\max \left(K_{\phi}(h), \frac{c_{\nu}}{2^{2 \nu}}\|\phi\|_{[0, h], \infty}\right)$ und $c_{\nu}=1 \mathrm{im}$ Clenshaw-Curtis-Fall und $c_{\nu}=2^{\nu}$ im Fall $m_{j}=2^{j-1}+1,1 \leq j \leq q-d+1$, definiert ist.

Beweis: Wir berechnen für den Fall $m_{1}=2$ zunächst

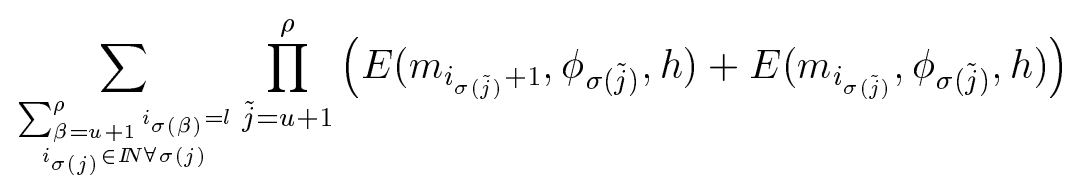

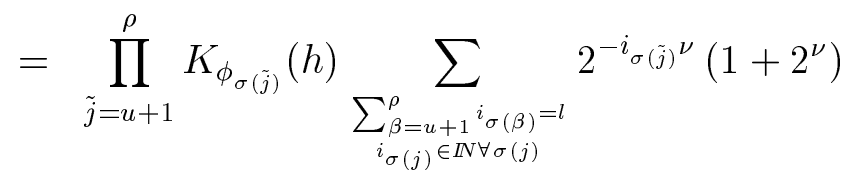

$$
\begin{aligned}
& =\prod_{\tilde{j}=u+1}^{\rho} K_{\phi_{\sigma(\tilde{j})}}(h)\left(1+2^{\nu}\right)^{\rho-u} 2^{-l \nu}\left(\begin{array}{c}
l-1 \\
\rho-u-1
\end{array}\right) .
\end{aligned}
$$

Damit gilt

$$
\begin{aligned}
& \sum_{\rho=0}^{d-1} \frac{1}{\rho !} \prod_{\alpha=\rho+2}^{d}\left\|\phi_{\alpha}\right\|_{[0, h], \infty} \sum_{u=0}^{\rho-1}\left(\begin{array}{l}
\rho \\
u
\end{array}\right) \sum_{l=\rho-u}^{k-d} E\left(m_{k-d+1-l}, \phi_{\rho+1}, h\right) \sum_{\sigma \in S_{\rho}} . \\
& \cdot \sum_{j=0}^{u}\left(\begin{array}{l}
u \\
j
\end{array}\right) \prod_{r=1}^{j}\left\|\phi_{\sigma(r)}\right\|_{[0, h], \infty} \prod_{p=j+1}^{u} E\left(m_{1}, \phi_{\sigma(p)}, h\right) \cdot \\
& \cdot \sum_{\substack{\sum_{\beta=u+1}^{\rho} i_{\sigma(\beta)}=l \\
i_{\sigma(j)} \in \mathbb{N} \forall \forall(j)}} \prod_{\tilde{j}=u+1}^{\rho}\left(E\left(m_{i_{\sigma(\tilde{j})}+1}, \phi_{\sigma(\tilde{j})}, h\right)+E\left(m_{i_{\sigma(\tilde{j})}}, \phi_{\sigma(\tilde{j})}, h\right)\right) \\
& =2^{-(k-d+1) \nu} \sum_{\rho=0}^{d-1} \tilde{K}_{\phi, \rho} \sum_{u=0}^{\rho-1}\left(\begin{array}{l}
\rho \\
u
\end{array}\right) \sum_{l=\rho-u}^{k-d} \sum_{j=0}^{u}\left(\begin{array}{l}
u \\
j
\end{array}\right)\left(1+2^{\nu}\right)^{\rho-u}\left(\begin{array}{c}
l-1 \\
\rho-u-1
\end{array}\right) \\
& =2^{-(k-d+1) \nu} \sum_{\rho=0}^{d-1} \tilde{K}_{\phi, \rho} \sum_{u=0}^{\rho-1}\left(\begin{array}{l}
\rho \\
u
\end{array}\right) \sum_{l=\rho-u}^{k-d} 2^{u}\left(1+2^{\nu}\right)^{\rho-u}\left(\begin{array}{c}
l-1 \\
\rho-u-1
\end{array}\right) \\
& =2^{-(k-d+1) \nu} \sum_{\rho=0}^{d-1} \tilde{K}_{\phi, \rho} \sum_{u=0}^{\rho-1}\left(\begin{array}{l}
\rho \\
u
\end{array}\right)\left(\begin{array}{c}
k-d \\
\rho-u
\end{array}\right) 2^{u}\left(1+2^{\nu}\right)^{\rho-u}
\end{aligned}
$$


und wir folgern

$$
\begin{aligned}
E_{d}^{\mathcal{S}, \otimes}(k, \Phi, h) & \leq 2^{-(k-d+1) \nu} \sum_{\rho=0}^{d-1} \tilde{K}_{\phi, \rho} \sum_{u=0}^{\rho}\left(\begin{array}{c}
\rho \\
u
\end{array}\right)\left(\begin{array}{c}
k-d \\
\rho-u
\end{array}\right) 2^{u}\left(1+2^{\nu}\right)^{\rho-u} \\
& \leq 2^{-(k-d+1) \nu} \sum_{\rho=0}^{d-1} \tilde{K}_{\phi, \rho} M_{\rho, \nu} \sum_{u=0}^{\rho}\left(\begin{array}{c}
\rho \\
u
\end{array}\right)\left(\begin{array}{c}
k-d \\
\rho-u
\end{array}\right) \\
& =2^{-(k-d+1) \nu} \sum_{\rho=0}^{d-1} \tilde{K}_{\phi, \rho} M_{\rho, \nu}\left(\begin{array}{c}
k-d+\rho \\
\rho
\end{array}\right) \\
& \leq 2^{-(k-d+1) \nu} \max _{0 \leq \rho \leq d-1}\left(M_{\rho, \nu} \tilde{K}_{\phi, \rho}\right) \sum_{\rho=0}^{d-1}\left(\begin{array}{c}
k-d+\rho \\
\rho
\end{array}\right) .
\end{aligned}
$$

Den zweiten Teil beweisen wir anfangs analog, es ist

$$
\begin{aligned}
& E_{d}^{\mathcal{S}, \otimes}(k, \Phi, h) \\
& \leq \sum_{\rho=0}^{d-1}\|\phi\|_{[0, h], \infty}^{d-\rho-1} 2^{-(k-d+1) \nu}\left(\sum_{u=0}^{\rho-1}\left(\begin{array}{l}
\rho \\
u
\end{array}\right)\left(1+2^{\nu}\right)^{\rho-u} \sum_{j=0}^{u}\left(\begin{array}{l}
u \\
j
\end{array}\right) K_{\phi}^{\rho-j+1}(h)\|\phi\|_{[0, h], \infty}^{j}+\right. \\
& \left.+\sum_{l=\rho-u}^{k-d}\left(\begin{array}{c}
l-1 \\
\rho-u-1
\end{array}\right) \sum_{j=0}^{\rho}\left(\begin{array}{l}
\rho \\
j
\end{array}\right) K_{\phi}^{\rho-j+1}(h)\|\phi\|_{[0, h], \infty}^{j}\right) \\
& =\sum_{\rho=0}^{d-1}\|\phi\|_{[0, h], \infty}^{d-\rho-1} 2^{-(k-d+1) \nu}\left(\sum_{u=0}^{\rho}\left(\begin{array}{l}
\rho \\
u
\end{array}\right)\left(\begin{array}{c}
k-d \\
\rho-u
\end{array}\right)\left(1+2^{\nu}\right)^{\rho-u} \sum_{j=0}^{u}\left(\begin{array}{l}
u \\
j
\end{array}\right) K_{\phi}^{\rho-j+1}(h)\|\phi\|_{[0, h], \infty}^{j}\right) \\
& =\sum_{\rho=0}^{d-1}\|\phi\|_{[0, h], \infty}^{d-\rho-1} 2^{-(k-d+1) \nu} K_{\phi}^{\rho+1}(h)\left(\sum_{u=0}^{\rho}\left(\begin{array}{c}
\rho \\
u
\end{array}\right)\left(\begin{array}{c}
k-d \\
\rho-u
\end{array}\right)\left(1+2^{\nu}\right)^{\rho-u}\left(1+\frac{\|\phi\|_{[0, h], \infty}}{K_{\phi}(h)}\right)^{u}\right) ;
\end{aligned}
$$

weiterhin gilt

$$
\begin{aligned}
& \sum_{u=0}^{\rho}\left(\begin{array}{l}
\rho \\
u
\end{array}\right)\left(\begin{array}{l}
k-d \\
\rho-u
\end{array}\right)\left(1+2^{\nu}\right)^{\rho-u}\left(1+\frac{\|\phi\|_{[0, h], \infty}}{K_{\phi}(h)}\right)^{u} \\
& \quad=\sum_{u=0}^{\rho}\left(\begin{array}{l}
\rho \\
u
\end{array}\right)\left(1+2^{\nu}\right)^{\rho}\left(\begin{array}{l}
k-d \\
\rho-u
\end{array}\right) \max _{0 \leq u_{0} \leq \rho}\left(\frac{K_{\phi}(h)+\|\phi\|_{[0, h], \infty}}{K_{\phi}(h)\left(1+2^{\nu}\right)}\right)^{u_{0}} \\
& \quad \leq \sum_{u=0}^{\rho}\left(\begin{array}{l}
\rho \\
u
\end{array}\right)\left(1+2^{\nu}\right)^{\rho}\left(\begin{array}{l}
k-d \\
\rho-u
\end{array}\right)\left(\frac{K_{\phi}(h)+2^{\nu} C_{\phi, \nu}}{K_{\phi}(h)\left(1+2^{\nu}\right)}\right)^{\rho} \\
& \quad=K_{\phi}^{-\rho}(h) \sum_{u=0}^{\rho}\left(\begin{array}{l}
\rho \\
u
\end{array}\right)\left(\begin{array}{l}
k-d \\
\rho-u
\end{array}\right)\left(\frac{K_{\phi}(h)+2^{\nu} C_{\phi, \nu}}{\|\phi\|_{[0, h], \infty}}\right)^{\rho}
\end{aligned}
$$

und wir folgern

$$
E_{d}^{\mathcal{S}, \otimes}(k, \Phi, h) \leq \sum_{\rho=0}^{d-1}\|\phi\|_{[0, h], \infty}^{d-1} 2^{-(k-d+1) \nu} K_{\phi}(h)\left(\begin{array}{c}
k-d+\rho \\
\rho
\end{array}\right)\left(\frac{K_{\phi}(h)+2^{\nu} C_{\phi, \nu}}{\|\phi\|_{[0, h], \infty}}\right)^{d-1}
$$




$$
=\sum_{\rho=0}^{d-1} 2^{-(k-d+1) \nu} K_{\phi}(h)\left(\begin{array}{c}
k-d+\rho \\
\rho
\end{array}\right)\left(K_{\phi}(h)+2^{\nu} C_{\phi, \nu}\right)^{d-1} .
$$

Der Clenshaw-Curtis-Fall folgt durch Multiplikation der Konstanten $K_{\phi_{j}}, 1 \leq j \leq d$, des vorangegangenen Ergebnisses mit $2^{\nu}$.

Die Schranken bekommen in Abhängigkeit von der Punktanzahl des dünnen Gitters folgende Gestalt:

Korollar 5.32 Sei $n=n(q, d)$ die Anzahl der Datenpunkte des dünnen Gitters $H_{q, d}^{C C}$ oder $H_{q, d}^{C C 2}$. Dann gilt unter den Voraussetzungen von Korollar 5.31

$$
E_{d}^{\mathcal{S}, \otimes}(q, \Phi, h) \leq \frac{K_{d, \Phi}(h)}{(d-1) !^{(\nu+1}} n^{-\nu}(2 d+\log (n))^{(d-1)(\nu+1)}
$$

mit

$$
K_{d, \Phi}(h):=\left\{\begin{array}{ll}
K_{\phi}(h)\left(K_{\phi}(h)+2^{\nu} C_{\phi, \nu}\right)^{d-1} & : \quad \phi_{1}=\ldots=\phi_{d}=\phi \\
\max _{0 \leq \rho \leq d-1}\left(M_{\rho, \nu} \tilde{K}_{\rho, d}(h)\right) & : \quad \text { sonst }
\end{array} .\right.
$$

Dieses Ergebnis ist auf jede glatte Funktion anwendbar:

Satz 5.33 Es gelten die Voraussetzungen von Korollar 5.31. Dann folgt für jede Basisfunktion $\Phi=\phi_{1} \otimes \ldots \otimes \phi_{d}$ mit $\phi_{j} \in C^{\nu}$ für $1 \leq j \leq d$ auf dem gestörten dünnen Clenshaw-Curtis-Gitter $L_{q, d}^{C C 2}$ und für alle $f \in \mathcal{N}_{\Phi}$ die Fehlerabschätzung

$$
\left\|f-s_{f, \Phi, X}\right\|_{\infty} \leq C_{\nu, d, \Phi}(h) n^{-\frac{\nu}{2}}(2 d+\log (n))^{\frac{(d-1)(\nu+1)}{2}}\|f\|_{\Phi}
$$

mit

$$
C_{\nu, d, \Phi}(h)=\frac{\left(1+c_{2}\right) c}{(d-1) !^{\frac{\nu+1}{2}}}\left(c^{2}+\max \left(c^{2}, \tilde{c}_{\nu} \max _{1 \leq j \leq d}\left\|\phi_{j}\right\|_{[0, h], \infty}\right)\right)^{\frac{d-1}{2}} .
$$

Gilt $\nu \geq 1$, so folgern wir

$$
\left\|f-s_{f, \Phi, X}\right\|_{\infty} \leq \tilde{C}_{\nu, d, \Phi}(h) n^{-\frac{\nu}{2}-\frac{1}{2 d}}(2 d+\log (n))^{\frac{(d-1)(\nu+1)}{2}+\frac{d-1}{2 d}}\|f\|_{\Phi}
$$

mit

$$
\tilde{C}_{\nu, d, \Phi}(h)=\frac{\left(1+c_{2}\right) c 2^{\frac{1}{2 d}} \sqrt{d}}{(d-1) !^{\frac{\nu+1}{2}}+\frac{1}{2 d}}\left(c^{2} h+\max \left(c^{2} h, \tilde{c}_{\nu} \max _{1 \leq j \leq d}\left\|\phi_{j}\right\|_{[0, h], \infty}\right)\right)^{\frac{d-1}{2}}
$$

mit

$$
\tilde{c}_{\nu}= \begin{cases}2^{-2 \nu} & : \quad X=L_{q, d}^{C C} \\ 1 & : \quad X=L_{q, d}^{C C 2}\end{cases}
$$


Beweis: Wir nutzen Korollar 5.31 und ein Resultat von Jackson. Es gilt für $l \geq \nu$

$$
E\left(l, \phi_{j}, h\right) \leq c_{1, \phi_{j}}\left(\frac{h}{l}\right)^{\nu}
$$

und damit auch für $l<\nu$

$$
E\left(l, \phi_{j}, h\right) \leq c_{2, \phi_{j}}\left(\frac{h}{l}\right)^{l} \leq c_{2, \phi_{j}} l^{-\nu} l^{\nu-l} \leq c_{2, \phi_{j}} l^{-\nu} \nu^{\nu} .
$$

Insgesamt folgt

$$
E\left(l, \phi_{j}, h\right) \leq c_{3, \phi_{j}} l^{-\nu}
$$

für allgemeine $\nu$ und

$$
E\left(l, \phi_{j}, h\right) \leq c_{3, \phi_{j}} h l^{-\nu}
$$

für $\nu \geq 1$.

Bemerkung 5.34 Unter Verwendung von Satz 5.33 erhalten wir im Vergleich mit Korollar 5.15 höhere Approximationsgüten. Interpolieren wir also mit TensorproduktBasisfunktionen, so bietet sich in jedem Fall die Nutzung von Satz 5.33 an.

\subsection{Auswertung}

\subsubsection{Wendlandfunktionen}

Wir wenden uns Tensorprodukt-Wendlandfunktionen zu. Dazu formulieren wir:

Definition 5.35 Seien $\phi_{s_{i}}:=\phi_{s_{i}+1, s_{i}}$ univariate Wendlandfunktionen. Dann bezeichnet $\Phi_{\mathbf{s}}=\phi_{s_{1}} \otimes \ldots \otimes \phi_{s_{d}}$ mit $\mathbf{s}=\left\{s_{1}, \ldots, s_{d}\right\} \in I_{0}^{d}$ die TensorproduktWendlandfunktion.

Diese Funktionen sind nach Satz 4.23 positiv definit. Es folgt 
Satz 5.36 Sei $\Phi:=\Phi_{\mathrm{s}}$ eine Tensorprodukt-Wendlandfunktion. Es gelten die Voraussetzungen von Satz 5.16 und 5.19. Dann folgern wir für jede Funktion $f \in \mathcal{N}_{\Phi}$

$$
\left\|f-s_{f, \Phi, X}\right\|_{\infty} \leq C_{\Phi, d} n^{-\left(s+\frac{1}{2 d}\right)}(2 d+\log (n))^{\left(s+\frac{1}{2}\right)(d-1)+\frac{d-1}{2 d}}\|f\|_{\Phi}
$$

mit der Basisfunktionen-Interpolante $s_{f, \Phi, X}$ auf dem Datensatz $X=L_{q, d}^{C C}$ oder $L_{q, d}^{C C 2}$ mit $|X|=n, s=\min _{1 \leq i \leq d} s_{i}, h \leq 1$

$$
\begin{aligned}
& C_{\Phi, d}(h)=\frac{\left(1+c_{2}\right) c 2^{\frac{1}{2 d}} \sqrt{d}}{(d-1) !^{s+\frac{1}{2}+\frac{1}{2 d}}}\left(c^{2} h+\max \left(c^{2} h, \tilde{c}_{s} \max _{1 \leq j \leq d} C_{s_{j}}\right)\right)^{\frac{d-1}{2}},
\end{aligned}
$$

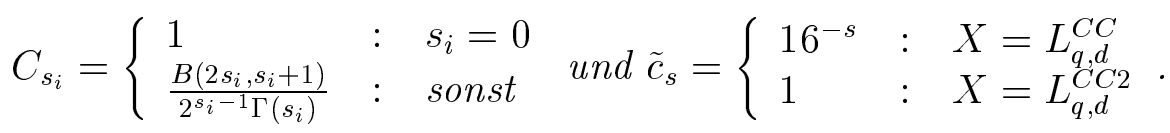

Beweis: Wir verwenden Satz 5.33 mit $\nu=2 s$ wegen $\phi_{s_{j}} \in C^{s_{j}}$. Da die Wendlandfunktion im Ursprung ihr Maximum $C_{s_{i}}$ annimmt, vgl. [37], folgern wir sofort die Behauptung.

Dieses Ergebnis ist der klassischen Fehlerschranke hinsichtlich der Komplexitätsproblematik deutlich überlegen. Allerdings erhalten wir auf dem gestörten dünnen Gitter eine etwas geringere Approximationsgüte als direkt auf dem dünnen Gitter, vgl. Satz 4.41 .

\subsubsection{Gaußglocken}

Aufgrund ihrer Funktionalgleichung stellen die Gaußglocken die einzigen Funktionen dar, welche gleichzeitig radial und tensoriert sind. Von daher ist jede Gaußglocke im Raum $\mathcal{F}_{1}^{l} \otimes \ldots \otimes \mathcal{F}_{1}^{l}$ für alle $l \in N_{0}$ enthalten und wir können Satz 5.33 auch im radialen Fall anwenden.

Satz 5.37 Es gelten die Voraussetzungen der Sätze 5.16 bzw. 5.19. Sei $\Phi:=\Phi_{\alpha}=e^{-\alpha\|\cdot\|^{2}}$ mit $\alpha \in \mathbb{R}_{+}$die Gaußglocke. Dann gilt für alle $l \in \mathbb{N}_{0}$, für alle $f \in \mathcal{N}_{\Phi}$ und ihre Interpolanten $s_{f, \Phi, X}$ auf dem gestörten dünnen Clenshaw-CurtisGitter $X=L_{q, d}^{C C}$ oder $L_{q, d}^{C C 2}$ die Fehlerschranke

$$
\left\|f-s_{f, \Phi, X}\right\|_{\infty} \leq \tilde{C}_{d, \Phi} n^{-l-\frac{1}{2 d}}(2 d+\log (n))^{(d-1)\left(l+\frac{1}{2}\right)+\frac{d-1}{2 d}}\|f\|_{\Phi}
$$


mit

$$
\tilde{C}_{d, \Phi}=\frac{\left(1+c_{2}\right) c 2^{\frac{1}{2 d}} \sqrt{d}}{(d-1) !^{l+\frac{1}{2}+\frac{1}{2 d}}}\left(c^{2} h+\max \left(c^{2} h, \tilde{c}_{l} \max _{1 \leq j \leq d}\left\|\phi_{j}\right\|_{[0, h], \infty}\right)\right)^{\frac{d-1}{2}}
$$

und $\tilde{c}_{l}=\left\{\begin{array}{ll}16^{-l} & : \quad X=L_{q, d}^{C C} \\ 1 & : \quad X=L_{q, d}^{C C 2}\end{array}\right.$.

Mit Satz 5.37 bekommen wir zwar eine Fehlerschranke, bei der der Fluch der Dimension erfolgreich bekämpft werden konnte; exponentielle Approximationsgüte erhalten wir auf dem chaotischen Datensatz jedoch noch nicht. Die Begründung liegt darin, daß wir hier im Vergleich zum klassischen Fall veränderte Lebesguesche Konstanten bekommen, deren Verhalten in Abhängigkeit von der Raumdimension noch ungeklärt ist. Folgt man der üblicherweise verwandten Auswertung der Lebesgueschen Konstanten [19], so wird die extreme Aufwendigkeit dieser Fragestellung deutlich.

Das folgende Ergebnis erhalten wir mit Korollar 5.28. Hier ist die Approximationsgüte noch abhängig von der Raumdimension und der Punktanzahl der Daten; von daher ist die Verwendung von Satz 5.37 vorzuziehen. Wir formulieren dennoch:

Satz 5.38 Für die Gaußglocke $\Phi:=\Phi_{\alpha}=e^{-\alpha\|\cdot\|^{2}}$ mit $\alpha \in \mathbb{R}_{+}$folgern wir unter den Voraussetzungen der Sätze 5.16 bzw. 5.19 und mit $h \leq \alpha^{-\frac{1}{2}}$

$$
\left\|f-s_{f, \Phi, X}\right\|_{\infty} \leq \frac{\left(1+c_{2}\right) 2^{\frac{d-1}{2}}}{\sqrt{(d-1) !}} C_{d, \alpha}^{C_{d}} n^{-\beta_{d, \alpha}(n)}(2 d+\log (n))^{(d-1)\left(\beta_{d, \alpha}(n)+\frac{1}{2}\right)}\|f\|_{\Phi},
$$

wobei

$$
\begin{aligned}
\beta_{d, \alpha}(n) & =-\frac{C_{d}}{d}-\log \left(C_{d, \alpha}\right)+\frac{1}{d} \log \left(n(2 d+\log (n))^{-(d-1)}\right), \\
C_{d, \alpha} & =\frac{\alpha \check{c} 2^{\frac{1}{d}} d}{(d-1) !} \quad \text { und } \\
C_{d} & =\left\{\begin{array}{lll}
\frac{3}{2} d+1-\log ((d-1) !) & : & X=L_{q, d}^{C C} \\
2 d+1-\log ((d-1) !) & : & X=L_{q, d}^{C C 2}
\end{array}\right.
\end{aligned}
$$

falls

$$
C_{d, \alpha}^{d} e^{C_{d}} \leq n(2 d+\log (n))^{-(d-1)}
$$

für alle $f \in \mathcal{N}_{\Phi}$. Dabei bezeichnet $s_{f, \Phi, X}$ die Interpolante an $f$ auf dem gestörten dünnen Clenshaw-Curtis-Gitter $X=L_{q, d}^{C C}$ oder $L_{q, d}^{C C 2}$; die Konstante $\check{c}$ entstammt Bedingung (24) aus Satz 5.8. 
Beweis: Unter Nutzung der Exponentialreihe folgern wir mit $\phi:=\phi_{\alpha}=e^{-\alpha|x|^{2}}$

$$
E(l, \phi, h) \leq \frac{\left(\alpha h^{2}\right)^{2\left\lfloor\frac{l-1}{2}\right\rfloor}}{l !} \leq\left(\alpha h^{2}\right)^{l-2}=\left(\alpha h^{2}\right)^{-2}\left(\alpha h^{2}\right)^{l}
$$

für alle $l \in I N$. Es folgt mit Korollar 5.28

$$
\begin{aligned}
E_{d}^{\mathcal{S}, \otimes}(k, \Phi, h) & \leq \frac{f(h)^{2(k-d-1)}}{g(h)}\left(\max \left(\frac{1}{f(h)^{2} g(h)}, 1\right)+\frac{1}{g(h)}\right)^{d-1}\left(\begin{array}{c}
k \\
d-1
\end{array}\right) \\
& \leq \frac{f(h)^{2(k-d-1)}}{g(h)^{d}}\left(1+f(h)^{-2}\right)^{d-1}\left(\begin{array}{c}
k \\
d-1
\end{array}\right) \\
& \leq\left(\alpha h^{2}\right)^{2 k-\left(4+1_{C C}\right) d} 2^{d-1}\left(\begin{array}{c}
k \\
d-1
\end{array}\right)
\end{aligned}
$$

mit den Bezeichnungen $f(h)=\left(\alpha h^{2}\right)^{-2}, g(h)$ wie in Korollar 5.28 und

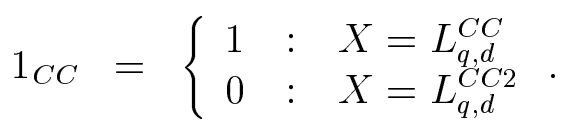

Damit gilt

$$
\begin{aligned}
& \left\|f-s_{f, \Phi, X}\right\|_{\infty} \\
& \leq 2^{\frac{d-1}{2}} \frac{G_{d}(n)^{\frac{1}{2}}}{\sqrt{(d-1) !}}\left(C_{d, \alpha} n^{-\frac{1}{d}} G_{d}(n)^{\frac{1}{d}}\right)^{k-\frac{(4+1, C C) d}{2}}\|f\|_{\Phi} \\
& \leq 2^{\frac{d-1}{2}} \frac{G_{d}(n)^{\frac{1}{2}}}{\sqrt{(d-1) !}}\left(C_{d, \alpha} n^{-\frac{1}{d}} G_{d}(n)^{\frac{1}{d}}\right)^{\log ((d-1) !)+\log (n)-\log \left(G_{d}(n)\right)+d 1_{C C}-1-\frac{(4+1) C C) d}{2}}\|f\|_{\Phi} \\
& =2^{\frac{d-1}{2}} \frac{G_{d}(n)^{\frac{1}{2}}}{\sqrt{(d-1) !}}\left(C_{d, \alpha} n^{-\frac{1}{d}} G_{d}(n)^{\frac{1}{d}}\right)^{-C_{d}+\log (n)-\log \left(G_{d}(n)\right)}\|f\|_{\Phi} \\
& =\frac{2^{\frac{d-1}{2}} C_{d, \alpha}^{C_{d}}}{\sqrt{(d-1) !}} n^{\log \left(C_{d, \alpha}\right)+\frac{C_{d}}{d}-\frac{1}{d} \log (n)+\frac{1}{d} \log \left(G_{d}(n)\right)} G_{d}(n)^{\frac{1}{2}-\frac{C_{d}}{d}+\frac{1}{d} \log (n)-\log \left(C_{d, \alpha}\right)-\frac{1}{d} \log \left(G_{d}(n)\right)}\|f\|_{\Phi}
\end{aligned}
$$

mit

$$
G_{d}(n)=(2 d+\log (n))^{d-1}
$$




\subsubsection{Inverse Multiquadrics}

Zunächst formulieren wir

Definition 5.39 Die Funktion $\Phi_{\mathbf{b}, \mathbf{c}}:=\phi_{\beta_{1}, c_{1}} \otimes \ldots \otimes \phi_{\beta_{d}, c_{d}}$ mit $\mathbf{b}=\left\{\beta_{1}, \ldots, \beta_{d}\right\} \in$ $\left(\mathbb{R}_{-} \backslash 2 \mathbb{N}\right)^{d}, \mathbf{c}=\left\{c_{1}, \ldots, c_{d}\right\} \in \mathbb{R}_{+}^{d}$ und $\phi_{\beta_{j}, c_{j}}\left(x_{j}\right)=\left(c_{j}^{2}+x_{j}^{2}\right)^{\frac{\beta_{j}}{2}}$ für $x_{j} \in \Omega_{j}$ heißst Inverser Tensorprodukt-Multiquadric.

Inverse Tensorprodukt-Multiquadrics sind nach Satz 4.23 positiv definit. Wir erhalten folgende Fehlerschranken:

Satz 5.40 Es gelten die Voraussetzungen von Satz 5.13. Sei $\Phi:=\Phi_{\mathbf{b}, \mathbf{c}}=\phi_{\beta_{1}, c_{1}} \otimes \ldots \otimes \phi_{\beta_{d}, c_{d}}$ mit $\mathbf{b}=\left\{\beta_{1}, \ldots, \beta_{d}\right\} \in\left(\mathbb{R}_{-} \backslash 2 \mathbb{N}\right)^{d}$ und $\mathbf{c}=\left\{c_{1}, \ldots, c_{d}\right\} \in \mathbb{R}_{+}^{d}$ ein Inverser Tensorprodukt-Multiquadric. Mit $h \leq 1$ folgt für $f \in \mathcal{N}_{\Phi}$, für die Interpolante $s_{f, \Phi, X}$ auf dem gestörten dünnen Gitter $X=L_{q, d}^{C C}$ oder $L_{q, d}^{C C 2}$ mit $|X|=n$ und für alle $l \in N_{0}$

$$
\left\|f-s_{f, \Phi, X}\right\|_{\infty} \leq \tilde{C}_{\beta, d, \Phi}(h) n^{-l-\frac{1}{2 d}}(2 d+\log (n))^{(d-1)\left(l+\frac{1}{2}\right)+\frac{d-1}{2 d}}
$$

mit

$$
\tilde{C}_{\beta, d, \Phi}(h)=\frac{\left(1+c_{2}\right) \sqrt{d}}{(d-1) !^{l+\frac{1}{2}+\frac{1}{2 d}}}\left(c^{2} h+\max \left(c^{2} h, \tilde{c}_{l} \max _{1 \leq j \leq d}\left(c_{j}^{2}+h^{2}\right)^{\frac{\beta}{2}}\right)\right)^{\frac{d-1}{2}}
$$

und $\tilde{c}_{l}=\left\{\begin{array}{ll}16^{-l} & : \quad X=L_{q, d}^{C C} \\ 1 & : \quad X=L_{q, d}^{C C 2}\end{array}\right.$.

Zum Beweis benutzen wir Satz 5.33. Ein weiteres Ergebnis liefert Korollar 5.28.

Wie auch im Fall der Gaußglocken können wir bei Verwendung der Inversen Multiquadrics dem Fluch der Dimension entgegenwirken, mit gleicher Begründung erhalten wir auch hier keine exponentielle Approximationsgüte. Weiterhin betrachten wir auch hier eine Folgerung aus Korollar 5.28, bei der die Approximationsgüte von der Raumdimension und der Anzahl der Punkte des zugrundegelegten Datensatzes abhängt:

Satz 5.41 Es gelten die Voraussetzungen von Satz 5.16 oder Satz 5.19. Sei $\Phi:=\Phi_{\mathbf{b}, \mathbf{c}}=\phi_{\beta_{1}, c} \otimes \ldots \otimes \phi_{\beta_{d}, c}$ mit $\mathbf{b}=\left\{\beta_{1}, \ldots, \beta_{d}\right\} \in\left(\mathbb{R}_{-} \backslash 2 \mathbb{N}\right)^{d}$ und 
$\mathbf{c}=\{c, \ldots, c\} \in \mathbb{R}_{+}^{d}$ ein Inverser Tensorprodukt-Multiquadric. Dann folgt für $h<\frac{c}{2}$, für $f \in \mathcal{N}_{\Phi}$, für ihre Interpolante $s_{f, \Phi, X}$ mit $X=L_{q, d}^{C C}$ oder $L_{q, d}^{C C 2}$ und $|X|=n$

$$
\begin{aligned}
& \left\|f-s_{f, \Phi, X}\right\|_{\infty} \\
& \quad \leq \frac{1+c_{2}}{\sqrt{(d-1) !}}\left(2^{\left|\frac{\beta}{2}\right|} c^{\frac{\beta}{2}}\right)^{\frac{d}{2}} C_{d, c}^{C_{d}} n^{-\beta_{d, c}(n)}(2 d+\log (n))^{(d-1)\left(\beta_{d, c}(n)+\frac{1}{2}\right)}\|f\|_{\Phi},
\end{aligned}
$$

wobei

$$
\begin{aligned}
\beta_{d, c}(n) & =\frac{C_{d}}{d}-\log \left(C_{d, c}\right)+\frac{1}{d} \log \left(n(2 d+\log (n))^{-(d-1)}\right), \\
C_{d, c} & =\frac{\check{c} 2^{1+\frac{1}{d}} d}{c(d-1) !} \quad \text { und } \\
C_{d} & =d\left(\frac{1_{C C}}{2}-1\right)+\log ((d-1) !)-1,
\end{aligned}
$$

falls

$$
C_{c, d}^{d} \leq n(2 d+\log (n))^{-(d-1)}
$$

erfüllt ist. Mit ̌́ bezeichnen wir die Konstante aus Bedingung (24) von Satz 5.8.

Beweis: Mit [33] folgern wir $\left\|\phi_{\beta, c}\right\|_{[0, h], \infty} \leq 2^{\left|\frac{\beta}{2}\right|} c^{\frac{\beta}{2}}$ und

$$
E\left(l, \phi_{\beta, c}, h\right) \leq 2^{\left|\frac{\beta}{2}\right|} \frac{c^{\frac{\beta}{2}+1}}{c-h}\left(\frac{2 h}{c}\right)^{l},
$$

aufgrund von Korollar 5.28 gilt dann

$$
\begin{aligned}
& E_{d}^{\mathcal{S}, \otimes}(k, \Phi, h) \\
& \leq\left(\frac{2 h}{c}\right)^{2(k-d)} u_{c}(h) 2^{\left|\frac{\beta}{2}\right|} \frac{c^{\frac{\beta}{2}+1}}{c-h}\left(\max \left(2^{\left|\frac{\beta}{2}\right|} \frac{c^{\frac{\beta}{2}+1}}{c-h} u_{c}(h), 2^{\left|\frac{\beta}{2}\right|} c^{\frac{\beta}{2}}\right)+\right. \\
& \left.+\left(\frac{2 h}{c}\right)^{2} u_{c}(h) 2^{\left|\frac{\beta}{2}\right|} \frac{c^{\frac{\beta}{2}+1}}{c-h}\right)^{d-1}\left(\begin{array}{c}
k \\
d-1
\end{array}\right) \\
& =\left(\frac{2 h}{c}\right)^{2(k-d)} u_{c}(h)\left(2^{\left|\frac{\beta}{2}\right|} \frac{c^{\frac{\beta}{2}+1}}{c-h}\right)^{d}\left(\max \left(u_{c}(h), 1-\frac{h}{c}\right)+\left(\frac{2 h}{c}\right)^{2}\right)^{d-1}\left(\begin{array}{c}
k \\
d-1
\end{array}\right) \\
& =\left(\frac{2 h}{c}\right)^{2(k-d)} u_{c}(h)\left(2^{\left|\frac{\beta}{2}\right|} \frac{c^{\frac{\beta}{2}+1}}{c-h}\right)^{d}\left(1+\left(\frac{2 h}{c}\right)^{2}\right)^{d-1}\left(\begin{array}{c}
k \\
d-1
\end{array}\right)
\end{aligned}
$$




$$
\begin{aligned}
& \leq\left(\frac{2 h}{c}\right)^{2(k-d)} u_{c}^{d}(h)\left(2^{\left|\frac{\beta}{2}\right|} c^{\frac{\beta}{2}}\right)^{d}\left(1+\left(\frac{2 h}{c}\right)^{2} \frac{1}{u_{c}(h)}\right)^{d-1}\left(\begin{array}{c}
k \\
d-1
\end{array}\right) \\
& \leq\left(\frac{2 h}{c}\right)^{2 k-d\left(2+1_{C C}\right)}\left(2^{\left|\frac{\beta}{2}\right|} c^{\frac{\beta}{2}}\right)^{d} 2^{d-1}\left(\begin{array}{c}
k \\
d-1
\end{array}\right)
\end{aligned}
$$

mit $u_{c}(h)=\left\{\begin{array}{lll}\frac{c}{2 h} & : & X=L_{q, d}^{C C} \\ 1 & : & X=L_{q, d}^{C C 2}\end{array} \quad\right.$ und $1_{C C}$ wie im Beweis von Satz 5.38. Wir definieren $G_{d}(n)$ ebenfalls wie im Beweis von Satz 5.38 und folgern dann

$$
\begin{aligned}
\| f & -s_{f, \Phi, X} \|_{\infty} \\
& \leq\left(1+c_{2}\right)\left(\frac{2 h}{c}\right)^{k-d\left(1+\frac{1}{2} 1_{C C}\right)}\left(2^{\left|\frac{\beta}{2}\right|} c^{\frac{\beta}{2}}\right)^{\frac{d}{2}} \frac{G_{d}(n)^{\frac{1}{2}}}{\sqrt{(d-1) !}} \\
& \leq K_{\beta, c, d}\left(C_{d, c} n^{-\frac{1}{d}} G_{d}(n)^{\frac{1}{d}}\right)^{\log ((d-1) !)+\log (n)+\left(d 1_{C C}-1\right)-\log \left(G_{d}(n)\right)-d\left(1+\frac{1}{2} 1 C C\right)} G_{d}(n)^{\frac{1}{2}} \\
& =K_{\beta, c, d}\left(C_{c, d} n^{-\frac{1}{d}} G_{d}(n)^{\frac{1}{d}}\right)^{C_{d}+\log (n)-\log \left(G_{d}(n)\right)} G_{d}(n)^{\frac{1}{2}} \\
& =K_{\beta, c, d} C_{d, c}^{C_{d}} n^{\log \left(C_{d, c}\right)-\frac{1}{d} \log (n)-\frac{C_{d}}{d}+\frac{1}{d} \log \left(G_{d}(n)\right)} G_{d}(n)^{-\log \left(C_{d, c}\right)+\frac{C_{d}}{d}+\frac{1}{d} \log (n)-\frac{1}{d} \log \left(G_{d}(n)\right)+\frac{1}{2}}
\end{aligned}
$$

mit

$$
K_{\beta, c, d}=\frac{1+c_{2}}{\sqrt{(d-1) !}}\left(2^{\left|\frac{\beta}{2}\right|} c^{\frac{\beta}{2}}\right)^{\frac{d}{2}} .
$$

\subsubsection{Multiquadrics}

Zunächst formulieren wir

Definition 5.42 Wir nennen die Funktion $\Phi_{\mathbf{b}, \mathbf{c}}: \Omega_{j} \rightarrow \mathbb{R}$ mit $\Phi_{\mathbf{b}, \mathbf{c}}:=\phi_{\beta_{1}, c_{1}} \otimes \ldots \otimes \phi_{\beta_{d}, c_{d}}$ mit $\mathbf{b}=\left\{\beta_{1}, \ldots, \beta_{d}\right\} \in\left(\mathbb{R}_{+} \backslash 2 \mathbb{N}\right)^{d}, \mathbf{c}=\left\{c_{1}, \ldots, c_{d}\right\} \in \mathbb{R}_{+}^{d}$ und $\phi_{\beta_{j}, c_{j}}\left(x_{j}\right)=(-1)^{\left\lceil\frac{\beta_{j}}{2}\right\rceil}\left(c_{j}^{2}+x_{j}^{2}\right)^{\frac{\beta_{j}}{2}}$ für $x_{j} \in \Omega_{j}$ Tensorprodukt-Multiquadric.

Der Smolyak-Polynomraum $\mathcal{S P}_{\tilde{q}, d}^{m_{1}, \ldots, m_{\tilde{q}-d+1}}$, der bei der Interpolation mit TensorproduktMultiquadrics verwandt wird, muß für $m_{1} \geq 1, m_{j} \geq 2^{j-1}+1,2 \leq j \leq \tilde{q}-d+1$ nach Lemma 5.1 die Bedingung $\tilde{q} \geq d+\max \left(0, \sum_{j=1}^{d}\left\lceil\frac{\beta_{j}}{2}\right\rceil-2\right)$ erfüllen. Wir erhalten folgende Fehlerschranken: 
Satz 5.43 Es gelten die Voraussetzungen der Sätze 5.16 und 5.19; Weiterhin sei $\Phi:=\Phi_{\mathbf{b}, \mathbf{c}}=\phi_{\beta_{1}, c_{1}} \otimes \ldots \otimes \phi_{\beta_{d}, c_{d}}$ mit $\mathbf{b}=\left\{\beta_{1}, \ldots, \beta_{d}\right\} \in\left(\mathbb{R}_{+} \backslash 2 \mathbb{N}\right)^{d}$ und $\mathbf{c}=\left\{c_{1}, \ldots, c_{d}\right\} \in \mathbb{R}_{+}^{d}$ ein Tensorprodukt-Multiquadric. Es gelte $h \leq 1$. Wir folgern für $f \in \mathcal{N}_{\Phi}$, für die Interpolante $s_{f, \Phi, X}$ auf dem Datensatz $X=L_{q, d}^{C C}$ oder $X=L_{q, d}^{C C 2}$ mit $|X|=n$

$$
\left\|f-s_{f, \Phi, X}\right\|_{\infty} \leq C_{\beta, d, \Phi}(h) n^{-\frac{\beta}{2}}(2 d+\log (n))^{\frac{(d-1)(\beta+1)}{2}}\|f\|_{\Phi}
$$

mit

$$
C_{\beta, d, \Phi}(h)=\frac{\left(1+c_{2}\right) c}{(d-1) !^{\frac{\beta+1}{2}}}\left(c^{2}+\max \left(c^{2}, \tilde{c}_{\beta} \max _{1 \leq j \leq d}\left(c_{j}^{2}+h^{2}\right)^{\frac{\beta_{j}}{2}}\right)\right)^{\frac{d-1}{2}}
$$

und

$$
\tilde{c}_{\beta}=\left\{\begin{array}{lll}
4^{-\beta} & : & X=L_{q, d}^{C C} \\
1 & : & X=L_{q, d}^{C C 2}
\end{array}\right.
$$

für $0<\beta<1$. Für $\beta \geq 1$ gilt

$$
\left\|f-s_{f, \Phi, X}\right\|_{\infty} \leq \tilde{C}_{\beta, d, \Phi}(h) n^{-\frac{\beta}{2}-\frac{1}{2 d}}(2 d+\log (n))^{\frac{(d-1)(\beta+1)}{2}+\frac{d-1}{2 d}}\|f\|_{\Phi}
$$

mit

$$
\tilde{C}_{\beta, d, \Phi}(h)=\frac{\left(1+c_{2}\right) \sqrt{d} c}{(d-1) ! \frac{\beta+1}{2}+\frac{1}{2 d}}\left(c^{2}+\max \left(c^{2}, \tilde{c}_{\beta} \max _{1 \leq j \leq d}\left(c_{j}^{2}+h^{2}\right)^{\frac{\beta_{j}}{2}}\right)\right)^{\frac{d-1}{2}} .
$$

Beweis: Wir benutzen Satz 5.33 wegen $\phi_{\beta_{j}, c_{j}} \in C_{\beta_{j}}$.

Da die Multiquadrics keine positiv definiten Funktionen darstellen, wurden sie in diesem Abschnitt zum ersten Mal zur multivariaten Interpolation mit Verwendung des Smolyak-Algorithmus eingesetzt. Auch in diesem Fall wurde dem komplexitätstheoretischen Problem entgegengewirkt, denn bei der klassischen Interpolation mit radialen Multiquadrics erhalten wir Fehlerabschätzungen der Gestalt

$$
\left\|f-s_{f, \Phi, X}\right\|_{\infty} \leq C_{\Phi, d} N^{-\frac{\beta}{2 d}}
$$

wobei $N$ die Anzahl der Punkte eines quasi-regulären Datensatzes auf einem beschränkten Gebiet darstellt. Gilt $\beta \leq 1$, so können wir abgesehen von einen logarithmischen Faktor sogar Approximationsgüten gewinnen, die vergleichbaren Größen aus der klassischen eindimensionalen Interpolation überlegen sind.

Wie auch bei den Inversen Multiquadrics erhalten wir wieder ein Ergebnis aus Korollar 5.28: 
Satz 5.44 Unter den Voraussetzungen von Satz 5.16 oder Satz 5.19 sei $\Phi:=\Phi_{\mathbf{b}, \mathbf{c}}=\phi_{\beta_{1}, c} \otimes \ldots \otimes \phi_{\beta_{d}, c}$ mit $\mathbf{b}=\left\{\beta_{1}, \ldots, \beta_{d}\right\} \in\left(\mathbb{R}_{+} \backslash 2 \mathbb{N}\right)^{d}$ und $\mathbf{c}=\{c, \ldots, c\} \in \mathbb{R}_{+}^{d}$ ein Tensorprodukt-Multiquadric. Es gil dann für $h<\frac{c}{2}$, für $f \in \mathcal{N}_{\Phi}$, für ihre Interpolante $s_{f, \Phi, X}$ mit $X=L_{q, d}^{C C}$ oder $L_{q, d}^{C C 2}$ und $|X|=n$

$$
\begin{aligned}
& \left\|f-s_{f, \Phi, X}\right\|_{\infty} \\
& \quad \leq \frac{1+c_{2}}{\sqrt{(d-1) !}}\left(2^{\left|\frac{\beta}{2}\right|} c^{\frac{\beta}{2}}\right)^{\frac{d}{2}} C_{d, c}^{C_{d}} n^{-\beta_{d, c}(n)}(2 d+\log (n))^{(d-1)\left(\beta_{d, c}(n)+\frac{1}{2}\right)}\|f\|_{\Phi},
\end{aligned}
$$

wobei

$$
\begin{aligned}
\beta_{d, c}(n) & =\frac{C_{d}}{d}-\log \left(C_{d, c}\right)+\frac{1}{d} \log \left(n(2 d+\log (n))^{-(d-1)}\right), \\
C_{d, c} & =\frac{\check{c} 2^{1+\frac{1}{d}} d}{c(d-1) !} \quad \text { und } \\
C_{d} & =d\left(\frac{1_{C C}}{2}-1\right)+\log ((d-1) !)-1,
\end{aligned}
$$

für

$$
C_{c, d}^{d} \leq n(2 d+\log (n))^{-(d-1)}
$$

erfüllt ist. Die Konstante ̌̌ stammt aus Bedingung (24) von Satz 5.8.

Der Beweis verläuft analog zu dem von Satz 5.41.

\subsubsection{Thin Plate Splines}

Wir definieren wieder

Definition 5.45 Sei $\phi_{\beta_{j}}: \Omega_{j} \rightarrow \mathbb{R}$ mit $\phi_{\beta_{j}}\left(x_{j}\right)=(-1)^{\frac{\beta_{j}}{2}+1}\left(x_{j}\right)^{\beta_{j}} \log \left(x_{j}\right)$ mit $x_{j} \in \Omega_{j}, \beta_{j} \in 2 \mathbb{N}_{0}$ und $1 \leq j \leq d$ ein Thin Plate Spline. Dann heißt die d-variate Funktion $\Phi_{\mathbf{b}}=\phi_{\beta_{1}} \otimes \ldots \otimes \phi_{\beta_{d}}$ mit $\mathbf{b}=\left\{\beta_{1}, \ldots, \beta_{d}\right\} \in(2 I N)^{d}$ Tensorprodukt Thin Plate Spline.

Wollen wir bei der Interpolation mit Tensorprodukt Thin Plate Splines den SmolyakPolynomraum $\mathcal{S P}_{\tilde{q}, d}^{m_{1}, \ldots, m_{\tilde{q}-d+1}}$ nutzen, so muß $\tilde{q} \geq d+\max \left(\sum_{j=1}^{d} \frac{\beta_{j}}{2}-2\right)$ gelten. Es folgt 
Satz 5.46 Es seien die Voraussetzungen von Satz 5.16 bzw. von Satz 5.19 erfüllt. Sei $\Phi=\Phi_{\mathbf{b}}, \mathbf{b}=\left\{\beta_{1}, \ldots, \beta_{d}\right\} \in(2 \mathbb{N})^{d}$ ein Tensorprodukt Thin Plate Spline. Dann folgern wir für $f \in \mathcal{N}_{\Phi}$, ihre Interpolante $s_{f, \Phi, X}$ auf dem Datensatz $X=L_{q, d}^{C C}$ oder $L_{q, d}^{C C 2}$ mit $|X|=n, \beta:=\min _{1 \leq i \leq d} \beta_{i}>0$ und $h<2^{-\frac{1}{\beta}}$,

$$
\left\|f-s_{f, \Phi, X}\right\|_{\infty} \leq \tilde{C}_{\beta, d, \Phi}(h) n^{-\frac{\beta+1}{2}}(2 d+\log (n))^{\frac{(d-1)(\beta+2)}{2}}\|f\|_{\Phi}
$$

mit

$$
\tilde{C}_{\beta, d, \Phi}(h)=\frac{\left(1+c_{2}\right) d^{\frac{d}{2}} 2^{\frac{d-1}{2 d}}}{(d-1) !^{\frac{\beta+2}{2}}}\left(c^{2}+\max \left(c^{2}, \tilde{c}_{\beta} \log (h)\right)\right)^{\frac{d-1}{2}}
$$

und $\tilde{c}_{\beta}=\left\{\begin{array}{ll}4^{-\beta} & : \quad X=L_{q, d}^{C C} \\ 1 & : \quad X=L_{q, d}^{C C 2}\end{array}\right.$.

Beweis: Da die Funktion $\phi_{\beta_{i}}(r)=\left|r^{\beta_{i}} \log (r)\right|$ im Intervall $\left[0,2^{-\frac{1}{\beta_{i}}}\right]$ monoton anwächst, gilt $\left\|\phi_{\beta_{i}}\right\|_{[0, h], \infty} \leq h^{\beta} \log (h) \leq h \log (h)$. Damit folgern wir unter Verwendung von Satz 5.33

$$
\left\|f-s_{f, \Phi, X}\right\|_{\infty} \leq C_{\beta, d, \Phi}(h) n^{-\frac{\beta}{2}-\frac{1}{2 d}}(2 d+\log (n))^{\frac{(d-1)(\beta+1)}{2}+\frac{d-1}{2 d}}\|f\|_{\Phi}
$$

mit

$$
C_{\beta, d, \Phi}(h)=\frac{\left(1+c_{2}\right) \sqrt{d}}{(d-1) ! \frac{\beta+1}{2}+\frac{1}{2 d}} h^{\frac{d-1}{2}}\left(c^{2}+\max \left(c^{2}, \tilde{c}_{\beta} \log (h)\right)\right)^{\frac{d-1}{2}}
$$

aus Satz 5.33 folgt

$$
h^{\frac{d-1}{2}} \leq c \frac{2^{\frac{d-1}{2 d}} d^{\frac{d-1}{2}}}{(d-1) ! \frac{d-1}{2 d}} n^{-\frac{d-1}{2 d}}(2 d+\log (n))^{\frac{(d-1)^{2}}{2 d}}
$$

und zusammenfassend bekommen wir das Ergebnis.

Auch die tensorierten Thin Plate Splines werden an dieser Stelle erstmalig zur komplexitätsreduzierenden Interpolation nach Smolyak angewandt. Bei der klassischen Interpolation mit Thin Plate Splines $\Phi$ auf dem quasi-regulären Datensatz $X \subset \Omega$ bei beschränktem Gebiet $\Omega$ und $|X|=N$ kann die Norm des Fehlerfunktionals durch Schranken der Form

$$
\left\|f-s_{f, \Phi, X}\right\|_{\infty} \leq C_{\Phi, d} N^{\frac{\beta}{d}}
$$

abgeschätzt werden; für positive Werte von $\beta$ folgern wir also, daß wir abgesehen vom nicht so sehr wesentlichen logarithmischen Faktor im Vergleich zu den eindimensionalen klassischen Ergebnissen verbesserte Approximationsgüten gewinnen können. 


\subsubsection{Polynomials}

Definition 5.47 Seien $\phi_{\beta_{j}}: \Omega_{j} \rightarrow \mathbb{R}, 1 \leq j \leq d$, mit $\phi_{\beta_{j}}\left(x_{j}\right)=(-1)^{\frac{\beta_{j}}{2}} x_{j}^{\beta_{j}}$ für $x_{j} \in \Omega_{j}$ univariate Polynomials. Dann heißt die Funktion $\Phi_{\mathbf{b}}=\phi_{\beta_{1}} \otimes \ldots \otimes \phi_{\beta_{d}}$ mit $\mathbf{b}:=\left\{\beta_{1}, \ldots, \beta_{d}\right\} \in \mathbb{R}_{+}^{d}$ Tensor Polynomial.

Bei der Interpolation mit dieser Basisfunktion muß für den Smolyak-Polynomraum $\mathcal{S P}_{\tilde{q}, d}^{m_{1}, \ldots, m_{\tilde{q}-d+1}}$ die Bedingung $\tilde{q} \geq d+\max \left(0, \sum_{j=1}^{d}\left\lceil\frac{\beta_{j}}{2}\right\rceil-2\right)$ gelten.

Satz 5.48 Es seien die Voraussetzungen der Sätze 5.16 und 5.19 erfüllt; $\Phi_{\mathbf{b}}=\phi_{\beta_{1}} \otimes \ldots \otimes \phi_{\beta_{d}}$ mit $\mathbf{b}=\left\{\beta_{1}, \ldots, \beta_{d}\right\} \in \mathbb{R}_{+}^{d}$ sei ein Tensor Polynomial. Dann folgern wir für alle $f \in \mathcal{N}_{\Phi}$ und ihre Interpolante $s_{f, \Phi, X}$ mit $X=L_{q, d}^{C C}$ oder $X=L_{q, d}^{C C 2}$ mit $|X|=n$

$$
\left\|f-s_{f, \Phi, X}\right\|_{\infty} \leq \tilde{C}_{\beta, d, \Phi} n^{\frac{\beta+1}{2}}(2 d+\log (n))^{\frac{(d-1)(\beta+2)}{2}}\|f\|_{\Phi}
$$

mit

$$
\tilde{C}_{\beta, d, \Phi}=\frac{\left(1+c_{2}\right) c d^{\frac{d}{2}} 2^{\frac{d-1}{2 d}}}{(d-1) ! \frac{\beta+2}{2}}\left(c^{2}+\max \left(c^{2}, \tilde{c}_{\beta}\right)\right)^{\frac{d-1}{2}}
$$

mit $n=|X|$ und $\tilde{c}_{\beta}=\left\{\begin{array}{lll}4^{-\beta} & : & X=L_{q, d}^{C C} \\ 1 & : & X=L_{q, d}^{C C 2}\end{array}\right.$.

\section{Beweis:}

Der Beweis verläuft analog zu dem von Satz 5.46.

Im Fall der Tensor Polynomials erreichen wir hinsichtlich der Komplexitätsreduktion ebenfalls unser Ziel. Die Approximationsgüte kann sogar für jeden Wert von $\beta$ bis auf den logarithmischen Faktor im Vergleich zur eindimensionalen Interpolation mit radialen Polynomials verbessert werden, da wir im klassischen Fall Fehlerschranken der Gestalt

$$
\left\|f s_{f, \Phi, X}\right\|_{\infty} \leq C_{\Phi, d} N^{-\frac{\beta}{d}}
$$

für einen quasi-regulären Datensatz $X$ mit $|X|=N$ auf dem beschränkten Gebiet $\Omega$ vorliegen haben. Das gleiche Ergebnis erreichen wir, falls die zugrundegelegte Basisfunktion aus dem Tensorprodukt von univariaten Thin Plate Splines und Polynomials besteht. 


\section{Symbolverzeichnis}

\begin{tabular}{|c|c|}
\hline$d$ & Raumdimension \\
\hline$q$ & Parameter für den Smolyak-Algorithmus \\
\hline$\Omega, \Omega_{j}$ & Gebiet \\
\hline$\hat{\phi}$ & $\begin{array}{l}\text { Gewöhnliche oder verallgemeinerte Fouriertransformierte } \\
\text { der Funktion } \phi\end{array}$ \\
\hline$S_{k}$ & Gruppe der Permutationen der Zahlen $\{1, \ldots, k\}, k \in \mathbb{I}$ \\
\hline $\log$ & Logarithmus zur Basis 2 \\
\hline$E(k, \phi, \epsilon)$ & $\inf _{p \in \mathbb{P}_{k}^{1}}\|\phi-p\|_{[0, \epsilon], \infty}$ \\
\hline$E_{d}^{\mathcal{S}}(k, \Phi, \epsilon)$ & Vgl. d. Seiten 95, 107 \\
\hline$E_{Q, d}^{\mathcal{S}, \Delta}(k, \Phi, \epsilon)$ & Vgl. Seite 104 \\
\hline$X_{1} \otimes \ldots \otimes X_{d}$ & Tensorproduktraum, vgl. Seite 19 \\
\hline$\sum_{j=1}^{n} x_{j} \otimes y_{j}$ & Tensor, vgl. Seite 19 \\
\hline$X^{*}$ & Algebraischer Dualraum des Vektorraumes $X$ \\
\hline$A \otimes B$ & Tensorproduktoperator, vgl. Seite 23 \\
\hline$X_{1} \otimes_{\alpha} \ldots \otimes_{\alpha} X_{d}$ & Bezüglich der $\|\cdot\|_{\alpha, X_{1} \otimes \ldots \otimes X_{d}}$ abgeschlossener Tensorraum \\
\hline$A_{q, d}$ & Smolyak-Operator, vgl. d. Seiten 18, 27 \\
\hline $\mathcal{S} \mathcal{V}_{q, d}^{\mathcal{X}_{1}, \ldots, \mathcal{X}_{q-d+1}}$ & Smolyak-Vektorraum, vgl. Seite 27 \\
\hline$H_{q, d}$ & Dünnes Gitter, vgl. Seite 30 \\
\hline$n(q, d)$ & Anzahl der Punkte des dünnen Gitters \\
\hline$H_{q, d}^{C C}$ & Clenshaw-Curtis-Gitter, vgl. Seite 33 \\
\hline$H_{q, d}^{C C C 2}$ & Dünnes Gitter mit $m_{j}=2^{j-1}+1,1 \leq j \leq q-d+1$, vgl. Seite 33 \\
\hline$A_{q, d}\left(\phi_{1}, \ldots, \phi_{q-d+1}\right)$ & Smolyak-Funktion, vgl. Seite 41 \\
\hline & $k$-variater Tensorproduktoperator der Form $I_{1} \otimes \ldots \otimes I_{1}$ \\
\hline$L_{q, d}^{C C}, L_{q, d}^{C C 2}$ & Gestörtes dünnes Clenshaw-Curtis-Gitter, vgl. Seite 88 \\
\hline & Separationsdistanz, vgl. Seite 88 \\
\hline$h_{X}$ & Fülldistanz, vgl. Seite 88 \\
\hline
\end{tabular}

Normen und Skalarprodukte

$\|\cdot\|$
$\|\cdot\|_{\infty}$
$\|\cdot\|_{[a, b], \infty}$
$(\cdot, \cdot)_{L_{2}}$
$\|\cdot\|_{L_{p}}$
$\|\cdot\|_{X}$
$|\cdot|$
$|\cdot|_{d}$

Euklidische Norm

Maximumsnorm

Maximumsnorm auf dem Intervall $[a, b]$

$L_{2}$-Skalarprodukt

Norm des Raumes $L_{p}, 1 \leq p \leq \infty$

Norm auf dem Banachraum $X$

Norm mit $|x|=\left|x_{1}\right|+\ldots+\left|x_{d}\right|$ für $x=\left(x_{1}, \ldots, x_{d}\right) \in \mathbb{R}^{d}$

Norm, die ausschließlich auf Vektoren $j=\left(j_{1}, \ldots, j_{d}\right) \in \mathbb{N}^{d}$ angewandt wird mit $|j|_{d}=|j|$ 
$\begin{array}{ll}\|U\|_{X, Y} & \text { Norm eines Operators } U: X \rightarrow Y \text { mit Banachräumen } X \text { und } Y \\ \|U\|_{\phi, \mathcal{V}} & \text { Norm eines Operators } U: \mathcal{N}_{\phi} \rightarrow \mathcal{V} \text { mit Banachräumen } \mathcal{V} \\ (\cdot, \cdot)_{\Phi} & \text { Skalarprodukt im Native Space, vgl. Seite 13 } \\ \|\cdot\|_{\Phi} & \text { Norm bzw. Seminorm im Native Space } \\ \|\cdot\|_{\alpha, X_{1} \otimes \ldots \otimes X_{d}} & \text { Tensorraumnorm } \\ \|\cdot\|_{\alpha^{*}, X_{1}^{*} \otimes \ldots \otimes X_{d}^{*}} & \text { Duale Tensorraumnorm, vgl. Seite } 21 \\ \|\cdot\|_{\lambda, X_{1} \otimes \ldots \otimes X_{d}} & \text { Spezielle Tensorraumnorm, vgl. Seite } 20 \\ \|\cdot\|_{\gamma, X_{1} \otimes \ldots \otimes X_{d}} & \text { Spezielle Tensorraumnorm, vgl. Seite } 22 \\ \|\cdot\|_{\alpha_{p}, X_{1} \otimes \ldots \otimes X_{d}} & p \text {-Nuklearnorm, } 1 \leq p \leq \infty, \text { vgl. Seite } 22 \\ (\cdot, \cdot)_{\beta, H_{1} \otimes \ldots \otimes H_{d}} & \text { Tensor-Skalarprodukt, vgl. Seite } 26\end{array}$

Spezielle Begriffe aus der Theorie der Interpolation mit Basisfunktionen

$P_{x, X, u}$

$\delta_{x}, x \in \Omega$

$\delta_{(x)}$

$\lambda_{x, X, u}$

$L_{\mathcal{P}}(\Omega)$

$L\left(\mathcal{P}^{\perp}\right)$

$\mathcal{N}_{\Phi}(\Omega), \mathcal{N}_{\Phi}$

$\mathcal{N}_{\Phi, \mathcal{P}}(\Omega)$

$F_{\Phi}(\Omega), \mathcal{F}_{\Phi}(\Omega)$

$R_{\Phi, \Omega}$

$\mathcal{L}_{\mathcal{P}, \Phi}(\Omega)$

$\mathcal{N}_{\phi_{1}, \phi_{2}, \mathcal{P}_{1}, \mathcal{P}_{2}}^{\otimes}\left(\Omega_{1} \times \Omega_{2}\right)$

\section{Funktionenräume}

$\mathbb{P}_{m}^{d}$

$\mathcal{P}$

$\mathcal{S P}_{q, d}^{m_{1}, \ldots, m_{q-d+1}}$

$C(\Omega)$

$C_{c}(\Omega)$

$C^{l}(\Omega), C^{l}$

$C_{c}^{l}(\Omega), C_{c}^{l}$

$C_{d}^{r}$

$\mathcal{F}_{d}^{r}$

$\mathcal{C}_{d, c}^{r}(U), \mathcal{D}(U)$

$\mathcal{S}_{d}$
Power- oder Gütefunktion, vgl. Seite 13

Punktauswertungsfunktional mit $\delta_{x} f=f(x)$ für alle

Funktionen $f$ auf $\Omega$

Vgl. Seite 16

Vgl. Seite 12

Vgl. Seite 12

Vgl. Seite 13

Native Space, vgl. Seite 16

Native Space, vgl. Seite 17

Vgl. Seite 16

Vgl. Seite 17

Vgl. Seite 16

Tensor Native Space, vgl. Seite 68

Raum der Polynome in $d$ Variablen vom Gesamtgrad kleiner $m$ Allgemeiner Polynomraum

Smolyak-Polynomraum, vgl. Seite 43

Raum der stetigen Funktionen auf $\Omega$

Raum der stetigen Funktionen mit kompaktem Träger auf $\Omega$

Raum der $l$-fach stetig partiell differenzierbaren

Funktionen auf $\Omega$

Raum der $l$-fach stetig partiell differenzierbaren

Funktionen mit kompaktem Träger auf $\Omega$

Vgl. Seite 51

Vgl. Seite 51

Vgl. Seite 54

Vgl. Seite 54 
$L_{p}(\Omega) \quad$ Quotientenvektorraum der $\mathcal{L}_{p}$-integrierbaren Funktionen, $1 \leq p \leq \infty$

$\mathcal{F}_{d, L_{p}}^{r}(S)$

Vgl. d. Seiten 55,58

$\mathcal{W}_{d, L_{p}}^{r}(S)$

Vgl. Seite 58

$\mathcal{S}_{k}(X)$

Raum der polynomialen Splines, vgl. Seite 70

\section{Indexmengen}

$J_{k, d}$ vgl. Seite $31, J_{k, d}^{i}$ vgl. Seite $33, I_{q, d}, \tilde{J}_{q, d}$ und $K_{\tilde{q}, d}$ vgl. Seite 83. 


\section{Literatur}

[1] Abraham, R., Marsden, J.E., Ratiu, T., Manifolds, Tensor analysis, and Applications, Applied Mathematical Sciences, Vol. 75, second edition, Springer-Verlag, New York, 1988.

[2] Antosik, P., Mikusinski, J., Sikorski, R., Theory of distributions, The sequential approach, Elsevier Scientific Publishing Company, Amsterdam, PWN - Polish Scientific Publishers, Warschau, 1973.

[3] Aronszajn, N., Theory of reproducing kernels, Trans. Amer. Math. Soc. 68, pp. 337-404, 1950 .

[4] Ash, R.B., Measure, integration and functional analysis, Academic Press, New York, London, 1972.

[5] Barthelmann, V., Novak, E., Ritter, K., High dimensional polynomial interpolation on sparse grids, Vorabdruck, 1998.

[6] Bochner, S., Vorlesungen über Fouriersche Integrale. Akademische Verlagsgesellschaft mbH, Leipzig, 1932.

[7] Burenkov, V.I., Sobolev spaces on domains, Teubner Texte zur Mathematik, Band 137, Stuttgart/ Leipzig, 1998.

[8] Delvos, F.-J., D-variate boolean interpolation, Journal of Approximation Theory 34 (1982), pp. 99-114.

[9] Delvos, F.-J., und Schempp, W., Boolean methods in interpolation and approximation, Pitman Research Notes in Mathematics Series 230, Longman, Essex 1989.

[10] Forster, O., Analysis 3, Integralrechnung im $\mathbb{R}^{n}$ mit Anwendungen, 3. Auflage, Vieweg Verlag, Braunschweig/Wiesbaden, 1984.

[11] Schaback, R., Franke C., Solving Partial Differential Equations by Collocation using Radial Basic Functions, Applied Mathematics and Computation, Volume 93, Issue 1, June 1998, pp. 73-83.

[12] Gradshteyn, I.S., Ryzhik, I.M., Table of integrals, series, and products, Corrected and enlarged edition, Academic Press, New York 1980.

[13] Greub, W., Multilinear Algebra, 2nd Edition, Springer-Verlag, Berlin Heidelberg, 1978. 
[14] Hall, C.A., On error bounds for cubic spline interpolation, Journal of Approximation Theory 1 (1968), pp. 209-218.

[15] Iske, A., Charakterisierung bedingt positiv definiter Funktionen für multivariate Interpolationsmethoden mit radialen Basisfunktionen, Dissertation, Göttingen 1994.

[16] Koster, F., A proof of the consistency of the finite difference technique on sparse grids, Bericht Nr. 642 (2000), Universität Bonn.

[17] Lichnerowicz, A., Einführung in die Tensoranalysis, HochschultaschenbücherVerlag, Band 77, Bibliographisches Institut, Mannheim, 1966.

[18] Light, W.A., und Cheney, E.W., Approximation theory in tensor product spaces, In: A. Dold und B. Eckmann, eds., Lecture Notes in Mathematics 1169, Springer Verlag, Berlin/Heidelberg 1985.

[19] Madych, W.R., Nelson, S.A., Bounds on multivariate polynomials and exponential error estimates for multiquadric interpolation, Journal of Approximation Theory 70, pp. 94-112, 1992.

[20] Maurin, K., Methods of hilbert spaces, Monografie Matematyczne (45), Polish Scientific Publishers, Warschau, 1972.

[21] Meschkowski, H., Hilbertsche Räume mit Kernfunktionen, Die Grundlehren der Mathematischen Wissenschaften in Einzeldarstellungen, Band 113, Springer Verlag, Berlin/Göttingen/Heidelberg, 1962.

[22] Michelli, C.A., Interpolation of scattered data: distance matrices and conditionally positive definite functions, Constructive Approximation 2, pp. 11-22, 1986.

[23] Müller-Gronbach, T., Hyperbolic cross designs for approximation of random fields, Preprint 1811, Fachbereich Mathematik, TH Darmstadt 1996.

[24] Novak, E., High dimensional numerical problems, Nonlinear Analysis, Vol. 30(1996), pp. 1439-1446.

[25] Novak, E., Ritter, K., High dimensional integration of smooth functions over cubes, Numerische Mathematik 75, pp. 79-97.

[26] Novak, E., Ritter, K., The curse of dimension and a universal method for numerical integration, Multivariate Approximation and Splines, Nürnberger, G., Schmidt, J.W., Walz, G., eds., ISNM, 1997, pp. 177-188. 
[27] Novak, E., Ritter, K., Schmitt, R., Steinbauer, A., On a recent interpolatory method for high dimensional integration, Journal of Computational Applied Mathematics 112 (1999), pp. 215-228.

[28] Novak, E., Sloan, I. H., Wozniakowski, H., Tractability of tensor product linear operators, Journal of Complexity 13 (1997), pp. 387-418.

[29] Nürnberger, G., Approximation by Spline Functions, Springer Verlag, Berlin/Heidelberg, 1989.

[30] Ritter, K., Average case analysis of numerical problems, Habilitationsschrift, Erlangen, Dezember 1995.

[31] Saitoh, S., Theory of reproducing kernels and its applications, Longman Scientific and Technical, Essex, 1988.

[32] Schaback, R., Creating surfaces from scattered data using radial basis functions, in: Daehlen, M., Lyche, T., Schumaker, L.L., eds: Mathematical Methods for Curves and Surfaces, pp. 477-496.

[33] Schaback, R., Reconstruction of multivariate functions from scattered data, Manuskript, 1997.

[34] Schaback, R., Native Spaces for Radial Basis Functions I, Proceedings of Chamonix, A. Le Méhauté, C. Rabut, L.L. Schumaker (eds.), pp. 1-18, (1997).

[35] Schaback, R., Werner, H., Numerische Mathematik, Springer Verlag, 4-te Auflage, Berlin Heidelberg, 1992.

[36] Schatten, R., A Theory of Cross-Spaces, Princeton University Press, 1950. Reprinted by Kraus Reprint Corporation, Milwood, New York, 1965, 12, p. 186.

[37] Schreiber, A., Präkonditionierung bei Gaußglocken und Inversen Multiquadrics, Diplomarbeit, Göttingen, 1996.

[38] Schiekofer, T., Die Methode der finiten Differenzen auf dünnen Gittern zur Lösung elliptischer und parabolischer PDE's, Dissertation, Bonn 1998.

[39] Schumaker, L.L., Spline Functions: Basic Theory, Pure and Applied Mathematics, A Wiley-Interscience Series, New York/Chichester/Brisbane/Toronto, 1981.

[40] Smolyak, S. A., Quadrature and interpolation formulas for tensor products of certain classes of functions, Soviet Math. Dokl. 4, pp. 240-243. 
[41] Sprengel, F., Periodic interpolation and wavelets on sparse grids, Preprint 96/8, Universität Rostock.

[42] Strauss, H., On interpolation with products of positive definite functions, Numerical Algorithms 15 (1997), pp. 153-165.

[43] Strauss, H., On products of positive definite functions, Approximation Theory IX, Chui, K.C., Schumaker, L.L., eds., Vanderbilt University Press, Nashville, TN, (1988), pp. 1-3.

[44] Swartz, B., Varga, R.S., Error bounds for spline and L-spline interpolation, Journal of Approximation Theory, 6 (1972), pp. 6-49.

[45] Treves, F., Topological vector spaces, distributions and kernels, Academic Press, New York, London, 1967.

[46] Wasilkowski, G. W., und Woźniakowski, H., Explicit cost bounds of algorithms for multivariate tensor product problems, Journal of Complexity 11 (1995), pp. $1-56$.

[47] Wendland, H., Konstruktion und Untersuchung radialer Basisfunktionen mit kompaktem Träger, Dissertation, Göttingen 1996.

[48] Wloka, J., Partielle Differentialgleichungen, Sobolevräume und Randwertaufgaben, B.G. Teubner Verlag, Stuttgart, 1982.

[49] Wu, Z., Schaback, R., Local error estimates for radial basis function interpolation of scattered data, IMA Journal of Numerical Analysis 13 (1993), pp. 13-27. 


\section{A Anhang}

Dieser Abschnitt enthält einige Umformulierungen für solche Summen, die in dieser Arbeit häufig verwendet worden sind. Sie finden sich ohne Beweise auch in [12].

A.1 Für $k, j \in N_{0}, k \geq j$ gilt

$$
\sum_{i=j}^{k}\left(\begin{array}{l}
i \\
j
\end{array}\right)=\sum_{i=0}^{k-j}\left(\begin{array}{c}
j+i \\
j
\end{array}\right)=\left(\begin{array}{c}
k+1 \\
j+1
\end{array}\right) .
$$

Beweis: Im Fall $k=j$ ist nichts zu zeigen. Sei also $k>j$. Unter der Annahme, daß (A.1) für $k-1$ gilt, folgt

$$
\sum_{i=j}^{k}\left(\begin{array}{l}
i \\
j
\end{array}\right)=\left(\begin{array}{c}
k \\
j+1
\end{array}\right)+\left(\begin{array}{l}
k \\
j
\end{array}\right)=\left(\begin{array}{l}
k+1 \\
j+1
\end{array}\right)
$$

A.2 Seien $d \in \mathbb{N}, n \in \mathbb{N}_{0}$. Dann gilt

$$
\sum_{\substack{|i|=d+n \\
i \in N^{d}}} 1=\sum_{\substack{|i|=n \\
i \in N_{0}^{d}}} 1=\left(\begin{array}{c}
d+n-1 \\
d-1
\end{array}\right) .
$$

Beweis: Die Behauptung ist für $d=1$ klar; weiterhin sei sie für $d-1$ schon gezeigt. Dann folgern wir

$$
\begin{aligned}
\sum_{\substack{|i|=d+n \\
i \in N^{d}}} 1 & =\sum_{j=1}^{n+1} \sum_{\substack{|i|=d+n-j \\
i \in N^{d}}} 1=\sum_{j=0}^{n} \sum_{\substack{|i|=d-1+n-j \\
i \in N^{d}}} 1 \\
& =\sum_{j=0}^{n}\left(\begin{array}{c}
d-2+n-j \\
d-2
\end{array}\right)=\sum_{j=0}^{n}\left(\begin{array}{c}
d-2+j \\
d-2
\end{array}\right) ;
\end{aligned}
$$

nun benutzen wir (A.1).

A.3 Mit $d \in \mathbb{N}, n \in \mathbb{N}_{0}$ gilt

$$
\sum_{\substack{|i| \leq d+n \\
i \in N^{d}}} 1=\sum_{\substack{|i| \leq n \\
i \in \mathbb{N}_{0}^{d}}} 1=\left(\begin{array}{c}
d+n \\
d
\end{array}\right) .
$$


Beweis: Wir beweisen (A.3) mit Hilfe von (A.2) und (A.1):

$$
\sum_{\substack{|i| \leq d+n \\
i \in N^{d}}} 1=\sum_{j=0}^{n} \sum_{\substack{|i|=d+j \\
i \in N^{d}}} 1=\sum_{j=0}^{n}\left(\begin{array}{c}
d+j-1 \\
d-1
\end{array}\right)=\left(\begin{array}{c}
d+n \\
d
\end{array}\right) .
$$

A.4 Für $k \in \mathbb{N}_{0}$ gilt

$$
\sum_{\substack{|i| \leq k \\
i \in \mathbb{N}}}(-1)^{k-|i|}\left(\begin{array}{c}
d-1 \\
k-|i|
\end{array}\right)=1
$$

Beweis: Mit (A.2) folgern wir

$$
\begin{aligned}
\sum_{\substack{|i| \leq k \\
i \in \mathbb{N}_{0}}}(-1)^{k-|i|}\left(\begin{array}{c}
d-1 \\
k-|i|
\end{array}\right) & =\sum_{j=0}^{k} \sum_{\substack{i=j \\
i \in N_{0}}}(-1)^{k-j}\left(\begin{array}{l}
d-1 \\
k-j
\end{array}\right) \\
& =\sum_{j=0}^{k}(-1)^{k-j}\left(\begin{array}{c}
d-1+j \\
j
\end{array}\right)\left(\begin{array}{l}
d-1 \\
k-j
\end{array}\right) \\
& =\sum_{j=0}^{k}(-1)^{k-j}\left(\begin{array}{c}
k \\
j
\end{array}\right)\left(\begin{array}{c}
d-1+j \\
k
\end{array}\right) \\
& =\left.\sum_{j=0}^{k}\left(\begin{array}{l}
k \\
j
\end{array}\right)\left(\frac{(-1)^{d-1}}{k !} \frac{\partial^{k} x^{d-1+j}}{\partial x^{k}}\right)\right|_{x=-1} \\
& =\left.\frac{(-1)^{d-1}}{k !}\left(\frac{\partial^{k}\left(x^{d-1} \sum_{j=0}^{k}\left(\begin{array}{c}
k \\
j
\end{array}\right) x^{j}\right)}{\partial x^{k}}\right)\right|_{x=-1} \\
& =\left.\frac{(-1)^{d-1}}{k !}\left(x^{d-1}(x+1)^{k}\right)\right|_{x=-1} \\
& =\left.\frac{(-1)^{d-1}}{k !}\left(x^{d-1} k !\right)\right|_{x=-1} \\
& =1 .
\end{aligned}
$$


Eine weitere Beweisvariante besteht darin, Lemma 3.15 von Wasilkowski und Woźniakowski auszunutzen, denn für die Smolyakfunktion $\phi \otimes \ldots \otimes \phi$ gilt

$$
\phi \otimes \ldots \otimes \phi=A_{q, d}(\phi, \ldots, \phi)=\sum_{\substack{|i| \leq q \\
i \in \mathbb{N}_{0}}}(-1)^{q-|i|}\left(\begin{array}{c}
d-1 \\
q-|i|
\end{array}\right)(\phi \otimes \ldots \otimes \phi) .
$$

A.5 Seien $n, m \geq \rho$. Es gilt dann

$$
\sum_{j=0}^{\rho}\left(\begin{array}{c}
n \\
j
\end{array}\right)\left(\begin{array}{c}
m \\
\rho-j
\end{array}\right)=\left(\begin{array}{c}
n+m \\
\rho
\end{array}\right) .
$$

Beweis: Wir folgern

$$
\begin{aligned}
\sum_{j=0}^{\rho}\left(\begin{array}{c}
n \\
j
\end{array}\right)\left(\begin{array}{c}
m \\
\rho-j
\end{array}\right) & =\frac{1}{\rho !} \sum_{j=0}^{\rho}\left(\begin{array}{l}
\rho \\
j
\end{array}\right) \frac{n !}{(n-j) !} \frac{m !}{(m-\rho+j) !} \\
& =\left.\left.\frac{1}{\rho !} \sum_{j=0}^{\rho}\left(\begin{array}{c}
\rho \\
j
\end{array}\right)\left(\frac{\partial^{j}\left(x^{n}\right)}{\partial x^{j}}\right)\right|_{x=1}\left(\frac{\partial^{\rho-j}\left(x^{m}\right)}{\partial x^{\rho-j}}\right)\right|_{x=1} \\
& =\left.\frac{1}{\rho !}\left(\frac{\partial^{\rho}\left(x^{n} \cdot x^{m}\right)}{\partial x^{\rho}}\right)\right|_{x=1} \\
& =\left.\frac{1}{\rho !}\left(\frac{\partial^{\rho}\left(x^{n+m}\right)}{\partial x^{\rho}}\right)\right|_{x=1} \\
& =\frac{1}{\rho !} \frac{(n+m) !}{(n+m-\rho) !} \\
& =\left(\begin{array}{c}
n+m \\
\rho
\end{array}\right) .
\end{aligned}
$$




\section{LEBENSLAUF}

Persönliche DAten

Name Anja Schreiber

Geburtstag 11. Mai 1971

Geburtsort Uslar

\section{SCHULAUSBILDUNG}

August 1977 - Juli 1981 Grundschule Uslar

August 1981 - Juli 1983 Orientierungsstufe Uslar

August 1983 - Mai 1990 Gymnasium Uslar

08. Mai 1990

Abitur

Studium An Der Georg-August-Universität Göttingen

Oktober 1990 - Oktober 1992 Grundstudium Mathematik, Studienrichtung Wirtschaftsmathematik

Oktober 1992 - November 1996 Hauptstudium Mathematik, Studienrichtung Wirtschaftsmathematik

seit November $1996 \quad$ Promotionsstudiengang Mathematik mit

Nebenfächern Betriebswirtschaftslehre und Stochastik

STUdiEnABSCHLÜSSE

22. Oktober 1992 Vordiplom Mathematik,

Studienrichtung Wirtschaftsmathematik

07. November 1996 Hauptdiplom Mathematik,

Studienrichtung Wirtschaftsmathematik

Sonstiges

seit Juli 1997 Stipendium der Graduiertenförderung des Landes Niedersachsen 